University of Louisville

ThinkIR: The University of Louisville's Institutional Repository

\title{
The role of HSL7 in morphology and pathogenicity and its interaction with other signaling components in the plant pathogen Ustilago maydis.
}

Charles Ben Lovely 1977-

University of Louisville

Follow this and additional works at: https://ir.library.louisville.edu/etd

\section{Recommended Citation}

Lovely, Charles Ben 1977-, "The role of HSL7 in morphology and pathogenicity and its interaction with other signaling components in the plant pathogen Ustilago maydis." (2010). Electronic Theses and Dissertations. Paper 859.

https://doi.org/10.18297/etd/859

This Doctoral Dissertation is brought to you for free and open access by ThinkIR: The University of Louisville's Institutional Repository. It has been accepted for inclusion in Electronic Theses and Dissertations by an authorized administrator of ThinkIR: The University of Louisville's Institutional Repository. This title appears here courtesy of the author, who has retained all other copyrights. For more information, please contact thinkir@louisville.edu. 
THE ROLE OF HSL7 IN MORPHOLOGY AND PATHOGENICITY AND ITS INTERACTION WITH OTHER SIGNALING COMPONENTS IN THE PLANT

PATHOGEN USTILAGO MAYDIS

By

Charles Ben Lovely

B. S. Biology, B. A. Chemistry, University of Louisville, 2002

\begin{abstract}
A Dissertation
Submitted to the Faculty of the

College of Arts and Sciences of the University of Louisville

In Partial Fulfillment of the Requirements

For the Degree of
\end{abstract}

Doctor of Philosophy

Department of Biology

University of Louisville

Louisville, Kentucky

August 2010 

THE ROLE OF HSL7 IN MORPHOLOGY AND PATHOGENICITY AND ITS INTERACTION WITH OTHER SIGNALING COMPONENTS IN THE PLANT PATHOGEN USTILAGO MAYDIS

\author{
By \\ Charles Ben Lovely \\ B. S. Biology, B. A. Chemistry, University of Louisville, 2002 \\ A Dissertation Approved on
}

May 18, 2010

by the following Dissertation Committee:

Dissertation Director 


\section{ACKNOWLEDGEMENTS}

I like to express my deepest gratitude to my mentor Dr. Michael Perlin for his patience, guidance, and encouragement throughout my graduate career. I took a different path from most graduate students and am greatly indebted to his belief that I could become the researcher I am today. Though I am still a young scientist with so much more to learn I would be where I am today without his insight and development. I would also like to thank my committee members, Dr. Cynthia Corbitt, Dr. Steve Ellis, Dr. Ron Gregg, and Dr. David Schultz for their constructive criticisms.

I feel that I would not have made it through the day to day process of development in my graduate career without the help of my Goat Lab partners, Zhanyang Yu, Jinny Paul, Charu Agarwal, Cau Pham, David Myers, and Greg Shaw. Their support and friendship softened the arduous moments of my career and allowed them to pass without causing a breakdown.

I would also like to thank my family for their support and encouragement in my chosen career. Their tireless efforts to push me and make me work harder to reach my goals were priceless. I owe all of my thanks to my parents for relentless love and support throughout my life. Lastly, I would not be here today had it not been for my love, Gina Nobles. She focused me when I needed to be focused, she made me laugh when I needed to laugh, and was there to build me back up when I had crumbled. She is my focus and support. 


\begin{abstract}
THE ROLE OF HSL7 IN MORPHOLOGY AND PATHOGENICITY AND ITS INTERACTION WITH OTHER SIGNALING COMPONENTS IN THE PLANT

PATHOGEN USTILAGO MAYDIS.

C. Ben Lovely
\end{abstract}

May 18,2010

Dimorphism is a highly conserved process in fungi in which a transition between a unicellular, yeast-like growth form and either a unicellular or multicellular, filamentous growth form occurs in response to several different environmental cues. The phytopathogenic fungus Ustilago maydis undergoes the dimorphic transition from a yeast-like saprobic growth form to a unicellular, hyphal growth form in response to successful mating and subsequent host cues. In addition, U. maydis can also undergo haploid filament formation in response to several environmental cues including low ammonium conditions and growth in lipids or acid $\mathrm{pH}$. On solid media deficient in ammonium (SLAD), U. maydis produces a filamentous colony morphology, while in liquid low ammonium media the cells do not form filaments.

The p21-activated protein kinases (PAKs) play a substantial role in regulating the dimorphic transition in fungi. Many activities of the PAKs are relayed through the mitogen-activated protein kinase (MAPK) pathway. The PAK-like Ste20 homologue, Smu1, is required for a normal response to pheromone via up-regulation of pheromone 
expression, potentially through the MAPK pathway. Disrupting smul reduced this upregulation, with the effect more pronounced in the $a 2$ mating background. Our experiments suggest that Smul also plays a role regulating cell length and the filamentous response on solid SLAD media. Yeast two hybrid analysis identified the conserved protein-arginine methyltransferase, Hsl7, as a potential interacter of Smu1. Hs17 regulates cell length and the filamentous response to solid SLAD, yet in an opposite fashion to Smul.

Interestingly, simultaneous disruption of $h s l 7$ and overexpression of $s m u l$ leads to a hyper-filamentous response on solid SLAD. In addition, the double mutant strain also forms filaments in liquid SLAD, while neither single mutant nor wild type strains display this phenotype. A similar filamentous response in both solid and liquid SLAD was also observed in strains lacking another PAK-like protein kinase involved in cytokinesis and polar growth, Cla4. This was not observed in a strain deleted for the activator of Cla4, the Rho-like GTPase Rac1. My data suggest that Hsl7 may regulate cell cycle progression, while both Smul and Cla4 appear to be necessary for the filamentous response in $U$. maydis. 


\section{TABLE OF CONTENTS}

PAGE

ACKNOWLEDGEMENTS...............................................................

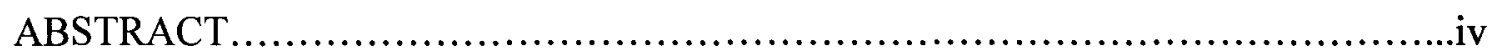

LIST OF TABLES .....................................................................

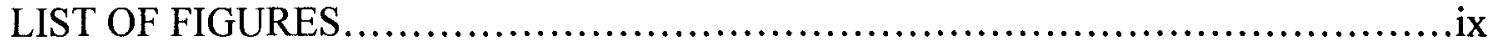

\section{CHAPTER}

I. GENERAL INTRODUCTION.............................................

History of Fungi in Disease and Agriculture.....................................

Dimorphism and Disease..................................................

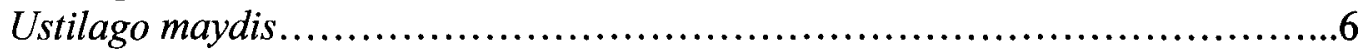

Regulating Dimorphism.................................................10

The cAMP Dependent Protein Kinase A Pathway ...............................11

The Mitogen-Activated Protein Kinase Pathway...................................14

Crosstalk between the MAPK and cAMP-PKA Pathways...........................18

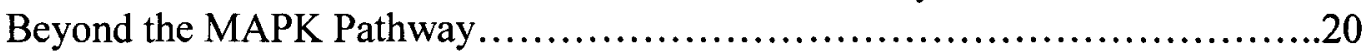

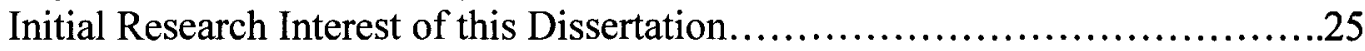

\section{CHAPTER}

II. THE METHYLTRANSFERASE HSL7 AND THE PAK KINASE SMU1 INTERACTIONS AFFECT CELL SIZE, THE FILAMENTOUS RESPONSE,

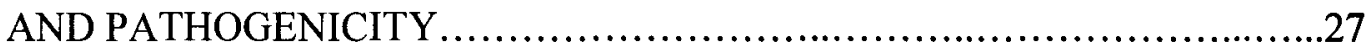

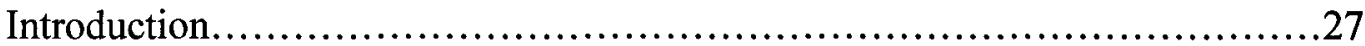

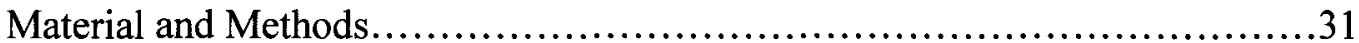

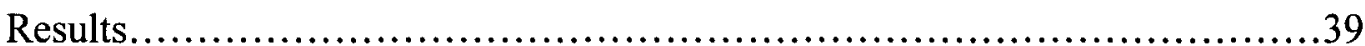

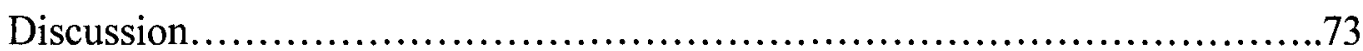

CHAPTER

III. CLA4, BUT NOT RAC1, REGULATES THE FILAMENTOUS RESPONSE TO LOW AMMONIUM CONDITIONS..............................................85

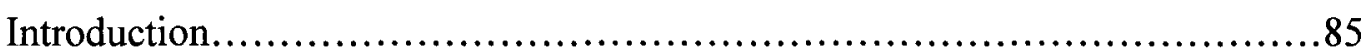

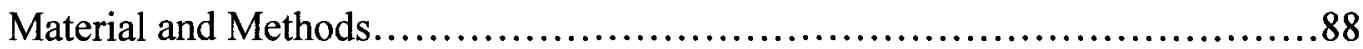

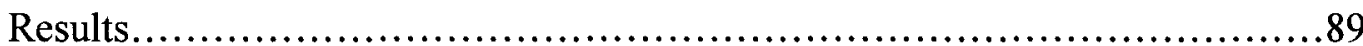

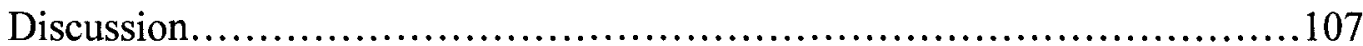

CHAPTER

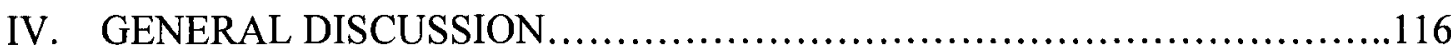




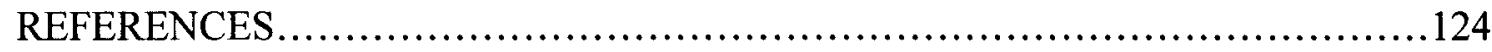

APPENDICES

I. HAPLO-INSUFFICIENCY IN THE HSL7 MUTANT BACKGROUND......140

Introduction and Discussion................................................140

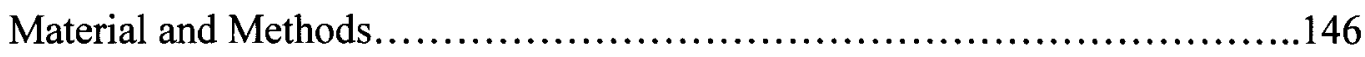

II. MATING-TYPE SPECIFIC CELL WALL SENSITIVITY IN A STRAIN

LACKING HSL7 ........................................................147

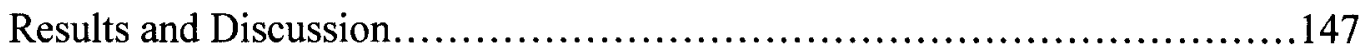

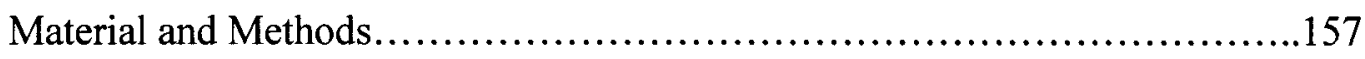

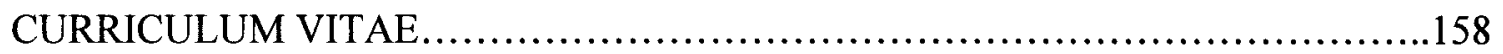




\section{LIST OF TABLES}

TABLE

PAGE

1. U. maydis strains and plasmids used in this study ..........................32

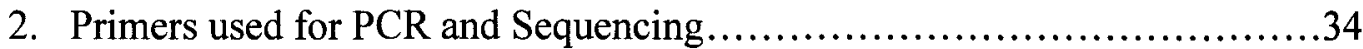

3. Measures of cell length across all strains....................................42

4. Mating-type specific cell length differences..............................42

5. Growth rate analysis of mutant strains....................................44

6. Cell length differences of the $\Delta_{810-2832} h s l 7 \mathrm{smul}{ }^{\text {Otef }}$ strains....................62

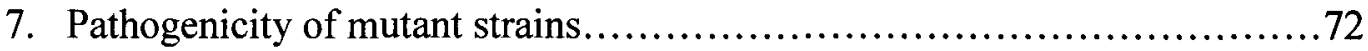

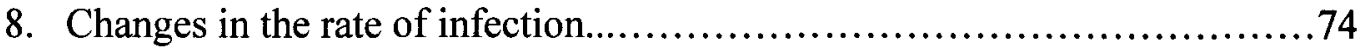

9. U. maydis strains and plasmids used in this study .............................88

10. Primers used for PCR and Sequencing....................................89

11. Measures of cell length across all strains...................................91

12. Mating-type specific cell length differences...............................91

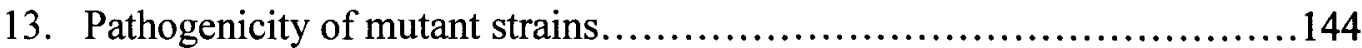

14. U. maydis strains and plasmids used in this study ..........................146

15. Measures of cell length of $M U M 11$ and $M U M 12$ strains...................149

16. Growth rate analysis of mutant strains..................................149

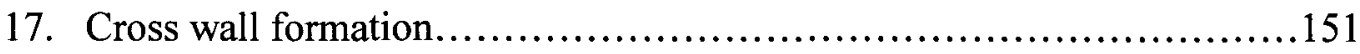

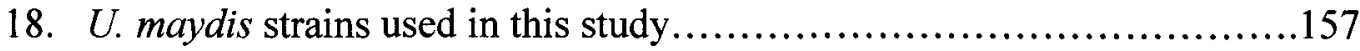




\section{LIST OF FIGURES}

FIGURE

PAGE

1. Representation of the life cycle of Ustilago maydis.........................7

2. General diagram of the cAMP dependent PKA pathway in S. cerevisiae ......12

3. Diagram of the $S$. cerevisiae MAPK pathway.............................15

4. Representation of cAMP-PKA and MAPK pathway from $U$. maydis.........19

5. Plate mating assays for $\Delta_{810-2832} h s l 7, h s l 7^{\text {Otef }}, \Delta s m u l, s m u l^{\text {tef }}, \Delta_{810-2832} h s l 7$ $\Delta s m u l, \Delta_{810-2832} h s l 7$ smul ${ }^{\text {Otef }}$ strains....................................... 41

6. Smul affects cell length but not cell wall localization......................43

7. smul mutant strains did not display any defects in colony morphology on rich media..........................................................46

8. Filamentous response to low ammonium conditions of $s m u l$ mutants........47

9. Hsl7 is a conserved protein arginine methyltransferase...................48

10. Hsl7 interacts with Smul in vitro.....................................50

11. Hsl7 affects cell length but not cell wall localization......................52

12. Disruption of $h s l 7$ leads to a directional growth phenotype, while overexpression of $h s l 7$ did not affect colony morphology on rich media.....54

13. Filamentous response to low ammonium conditions of $h s l 7$ mutants.........55

14. Disrupting smul in a $\Delta_{810-2832} h s l 7$ background does not rescue cell length increases observed in the $\Delta_{810-2832} h s l 7$ background, nor does it affect cell wall localization......................................................58

15. $\Delta_{810-2832} h s l 7 \Delta s m u l$ strains produce the directional growth phenotype on rich media......

16. $\Delta_{810-2832} h s l 7 \Delta s m u l$ mutants are hyper-filamentous on SLAD ...............60

17. $\Delta_{810-2832} h s l 7 s m u l^{\text {Otef }}$ cells are dramatically increased in cell length and the $a 2 b 2$ mating-type strain displays defects in cell separation 
18. The $a 2 b 2$ mating-type $\Delta_{810-2832} h s l 7 s m u l^{\text {Otef }}$ strain is multinucleic...........63

19. On rich media, the $\Delta_{810-2832} h s l 7 s m u l^{\text {Otef }}$ strains are filamentous.............65

20. On SLAD media, the $\Delta_{810-2832} h s l 7 s m u l^{\text {Otef }}$ strains are hyper-filamentous...66

21. $\Delta_{810-2832} h s l 7 \mathrm{smul}^{\text {Otef }}$ strains produce filaments in liquid SLAD and display defects in cell wall localization...........................................68

22. $\Delta_{810-2832} h s l 7, h s l 7^{\text {Otef }}, \Delta s m u l, s m u l^{\text {Otef }}$, and $\Delta_{810-2832} h s l 7 \Delta s m u l$ strains were stained with CFW, WGA, and Syto 11

23. The mating-type specific effects of $\Delta_{810-2832} h s l 7 s m u l^{\text {Otef }}$ strains in liquid SLAD...................................................................70

24. Disease symptom formation in various $U$. maydis mutant strains............73

25. Cla4 plays a role in cytokinesis and cell length...........................90

26. $\Delta c l a 4$ strains are multinucleic and exhibit chitin delocalization.............92

27. cla $^{\text {Otef }}$ strains did not exhibit any defects in cell wall localization when stained with CFW and WGA.............................................93

28. cla4 mutant strains are unaffected for colony morphology on rich media...95

29. On SLAD media, the $\Delta c l a 4$ strains display aberrant filament formation.....96

30. The albl cla $4^{\text {Otef }}$ mutant strain exhibits an increase in filamentation; however the $a 2 b 2 \mathrm{cla}^{\text {Otef }}$ mutant strain is reduced in the filamentous response on SLAD.

31. Acla4 strains produce filaments in liquid SLAD and display defects in cell wall localization.

32. $\Delta c l a 4$ strains display mating-type specific effects in liquid SLAD, while cla $4^{\text {Otef }}$ strains did not exhibit any defects in cell wall localization and nuclei number.................................................................100

33. Rac1 plays a role in cytokinesis and cell morphology....................101

34. Rac1 mutant strains differ in colony morphology on rich media...........102

35. The filamentous response to SLAD of the Racl mutant strains vary compared to wild type strains.

36. Racl mutant strains are unaffected in cell wall localization and nuclei number in SLAD media.

37. Colony morphology of Cla4 and Rac1 mutant strains grown on SLAD for 3 and 6 days post inoculation (dpi) 
38. Filamentous response to low ammonium conditions of the diploid mutant

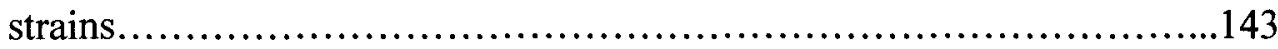

39. Disease symptom formation in the H-I $U$. maydis mutant strains...........145

40. $M U M$ strains display an increase in cell length and cross wall septa.......150

41. MUM strains exhibit a directional growth phenotype......................152

42. Filamentous response to low ammonium conditions of $h s l 7$ mutants.......153

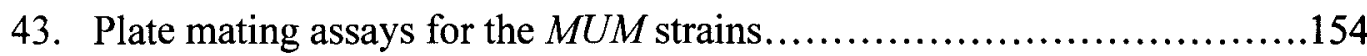

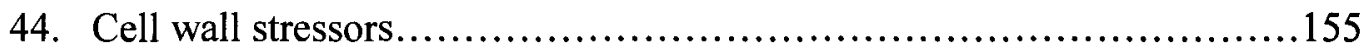

45. Rescue of CFW sensitivity by the osmotic stabilizer sorbitol...............156 


\section{CHAPTER I}

\section{HISTORY OF FUNGI IN DISEASE AND AGRICULTURE}

Fungi have played and continue to play an important role in life on this planet. Belonging to a kingdom separate from bacteria, plants, and animals, these eukaryotic organisms are comprised of single-cell species as well as multicellular species. Approximately 70,000 fungal species have been identified; however, estimates on the true number are much greater (Hawksworth, 1991). Found all over the world, these organisms fill about every niche available in the environment going mostly unnoticed as decomposers found in soil. In addition, many serve as symbionts with plants and animals, or even other fungi. However, several species act as opportunistic pathogens of plants and animals, as well. Historically, several pathogenic fungi have had impact on humans worldwide either directly though disease or indirectly by destroying agriculture. Aspergillus fumigatus, Candida albicans, Coccidiodes immitis, Cryptococcus neoformans, Histoplasma capsulatum, Paracoccidioides brasilinesis, and Pneumocystis carnii all can cause serious infections in humans and can lead to death. Treatments for many forms of cancer and the current AIDS epidemic are resulting in increases in fungal infections due to systemic immune suppression. Many of these species produce several compounds called secondary metabolites (mycotoxins) that are toxic to animals and are important in the virulence process. Aspergillus fumigatus produces several of these mycotoxins, including gliotoxin and sterigmatocystin that are important in virulence of 
this fungus (Bok and Keller, 2003). These toxins target cells in the immune system and suppress many of their functions or lead to outright apoptosis of several cell lines.

Several species that do not directly infect humans produce mycotoxins that, when ingested, are highly toxic. Amanita species produce amatoxins and are found throughout the world and have been important for many cultures for the religious ceremonies. The species Amanita muscaria was featured in the story Alice in Wonderland due to its purported hallucinogenic effects. Ergotism (Saint Anthony's fire) is another fungalbased affliction caused by ingesting rye contaminated with Claviceps purpurea and has lead to several outbreaks in human history. One of the first recorded outbreaks was in the Rhine Valley in Germany in 857 A.D. It has been proposed that the Salem witch trials in the late $17^{\text {th }}$ century were due to an ergotism outbreak (Shelton, 2001 [http:/www.plant.uga.edu/labrat/ergot.htm]). Many of these same groups of fungi produce other secondary metabolites that are of extreme importance to humans, especially antibiotics. Penicillium chrysogenum produces the first identified and widely used antibiotic, penicillin, while Cephalosporium acremonium produces cephalosporins. Cylindrocarpon lucidum and Tolypocladium inflatum both produce cyclosporin.

Several groups of fungi target many plant species important to agriculture or are of agricultural importance themselves. The great Irish potato famine in the mid $19^{\text {th }}$ century that resulted in the deaths of more than a million Irish was triggered by a potato blight (Phytophthora infestans) outbreak. Current losses (2009) due to potato blight are in the billions of dollars (International Potato Center [http://cipotato.org]). Several other fungal species (e.g., Ustilago spp., Magnoporthe grisea, Fusarium spp., Erysiphe spp., Aspergillus spp., C. purpurea) destroy billions of dollars of crops of several important 
crops (corn, wheat, barley, and rice), and can lead to mass starvations or other severe outcomes. Several fungal species are prized agricultural products. The common button mushroom, Agaricus bisporus, shiitake mushrooms, Lentinula edodes, oyster mushrooms, Pleurotus ostreatus, and truffles, Tuber melanosporum, are but a few edible mushroom species. Moreover, other species are used in the production of several different food stuffs. Blue cheeses are created by the addition of several different Penicillium species, while species of Saccharomyces, specifically Saccharomyces cerevisiae, are important for making many types of bread and alcoholic beverages. Aspergillus oryzae is necessary for the production of soy sauce and saki. Newer uses for fungi have been in the field of bioremediation where it was discovered that several species are able to degrade environmentally toxic compounds, i.e., herbicides/insecticides and fossil fuel byproducts, as well as removing heavy metals. Fungi are highly important in scientific research and studies in various species have lead to the understanding of several significant biological process like cell cycle regulation, genetic maintenance and expression, and pathogenesis. Fungal research continues to expand understanding of biology and its impact on humans.

\section{DIMORPHISM AND DISEASE}

Many fungal species display a phenomenon called dimorphism. Dimorphism is a highly conserved process in fungi in which a transition between a unicellular, yeast-like growth form and filamentous, sometimes multicellular, growth form occurs. In the unicellular growth form, fungi divide their nuclei mitotically then undergo cytokinesis resulting in complete, independent cells. The hallmark of filamentous growth is a tubelike structure which may be divided by septa into connected compartments. Albeit 
similar to filamentous growth, pseudohyphal growth differs in the form of the filament. In hyphal formation, the septated compartments are uniform and usually remain connected, open to one another through pores in the septal walls (Alexopoulos, et al., 1996). However, in pseudohyphal growth the connected cells undergo normal mitotic division but fail to separate and are reminiscent of individual cells joined end to end with no pores connecting the cells. In addition, while hyphae are a continuous tube in shape, pseudohyphae appear more similar to connected, independent cells. Moreover, both types of filaments can be invasive, allowing the fungus the ability to penetrate substrates on which they grow (Madhani and Fink, 1998).

C. albicans and S. cerevisiae, both of the same fungal family, Ascomycota, undergo different dimorphic transitions, where $C$. albicans produces true hyphae and $S$. cerevisiae produces pseudohyphae. Overall the regulation of this transition is similar between the two organisms; however the cues that trigger it can be dramatically different. C. albicans forms filaments in response to blood serum and a temperature change, while S.cerevisiae transitions from yeast-like cells to pseudohyphae under conditions of low ammonium. The process of dimorphism has been defined as a reversible process in which some fungal species can make a transition from yeast-like to hyphal growth and back (Nadal, et al., 2008). C. albicans can readily switch its growth between the yeastlike and hyphal forms and this ability is essential for virulence. However, in other fungi the switch is not reversible and once the switch occurs the organism has to complete its lifecycle. The plant pathogen Ustilago maydis must undergo mating and completes its lifecycle in the host plant, ultimately returning to its yeast-like phase upon germination of spores released from the plant (Nadal, et al., 2008). 
Fungi switch growth forms in response to various cues, including mating pheromone, environmental conditions, and host cues (in the case of pathogenic species). Depending on the species, several environmental cues can trigger this transition. It is posited that in response to environmental conditions $S$. cerevisiae undergoes the transition to pseudohyphal growth to search for nutrient rich, optimal substrates. Mucor species undergo a morphogenic transition in response to type of carbon source present and atmospheric oxygen levels. Yeast-like growth occurs only when a fermentable hexose is available and under anaerobic conditions (Orlowski, 1991). The human pathogens, Blastomyces dermatitidis, H. capsulatum, and C. albicans, all undergo a dimorphic transition when moving from $25^{\circ} \mathrm{C}$ to $37^{\circ} \mathrm{C}$, though both $B$. dermatitidis and $H$. capsulatum convert from a benign hyphal form to parasitic yeast-like form, whereas $C$. albicans switches from a yeast-like form to a pathogenic hyphal form (Sánchez-Martínez and Pérez-Martín, 2001). Additionally, C. albicans can undergo the yeast-like to hyphal transition in response to basic $\mathrm{pH}$, nutrient starvation, and the addition of serum. $U$. maydis undergoes the switch from yeast-like to hyphal growth in response to successful mating and subsequent host cues. The development of stable hyphal filaments occurs only in planta and appears to be dependent on signals from the host plant. In pathogenic dimorphic fungi, the ability to infect is linked to the dimorphic transition. Failure of any of the dimorphic pathogenic fungi previously mentioned to undergo a morphological transition attenuates their ability to infect their host species. In addition, U. maydis can also undergo haploid filament formation in response to several cues including low ammonium conditions and growth in lipids or acid $\mathrm{pH}$, and this is independent of mating and virulence. 


\section{Ustilago maydis}

Smut fungi, so named for the darkened spores they produce, were first identified in 1730 . They were initially described by Jethro Tull as "black stinking powder," though the causal agent was not recognized until the 1750's as coming from other infected, smut containing plants. Subsequent studies identified that spores produced in infected plants were able to cause smut disease in other plants (Money, 2006). Ustilago maydis (corn smut) is a basidiomycete fungus that infects maize (Zea mays) and teosinte (Euchlena mexicana) and was first described in 1815 by de Candolle. U. maydis is a plant pathogen but for centuries has been a delicacy (Banuett, 1995). Corn smut disease, or huitlacoche, originated in what is now Mexico and was believed to have mystic powers and be the food of the gods to the Aztecs (Specialty Produce [http://www.SpecialtyProduce.com]). Currently, it is a greatly desired commodity in Mexico, valued for its high nutritional value. Huitlacoche is a common staple of Mexican foods and has been cultivated as a cash crop, being potentially more valuable than maize itself (Plant and Fungi, Ustilago maydis [http://www.kew.org/plants-fungi/Ustilago-maydis.htm]). Though a delicacy in Mesoamerica from prehistoric times until now, it is a potent pathogen and can cost billions of dollars in preventative measures, i.e. fungicides and/or plant genetic modifications, and lost crop yields. Maize is the number one cultivated crop in the U.S. and U. maydis is considered an agricultural threat to these maize crops (USDA, 2009 [http://www.ers.usda.gov/briefing/corn/]). Breeding programs have lead to almost all corn in the U.S. being genetically identical, making the national crop of corn easily susceptible to a highly virulent disease. U. maydis as well as other corn diseases are of great concern to the U.S. both economically and agriculturally. However, this attitude 
has not stopped a small community of huitlacoche enthusiasts from trying to open American palates to this Mexican delicacy. They have even renamed it the Mexican truffle.

The life cycle of $U$. maydis (Fig. 1) is divided into three separate stages: a

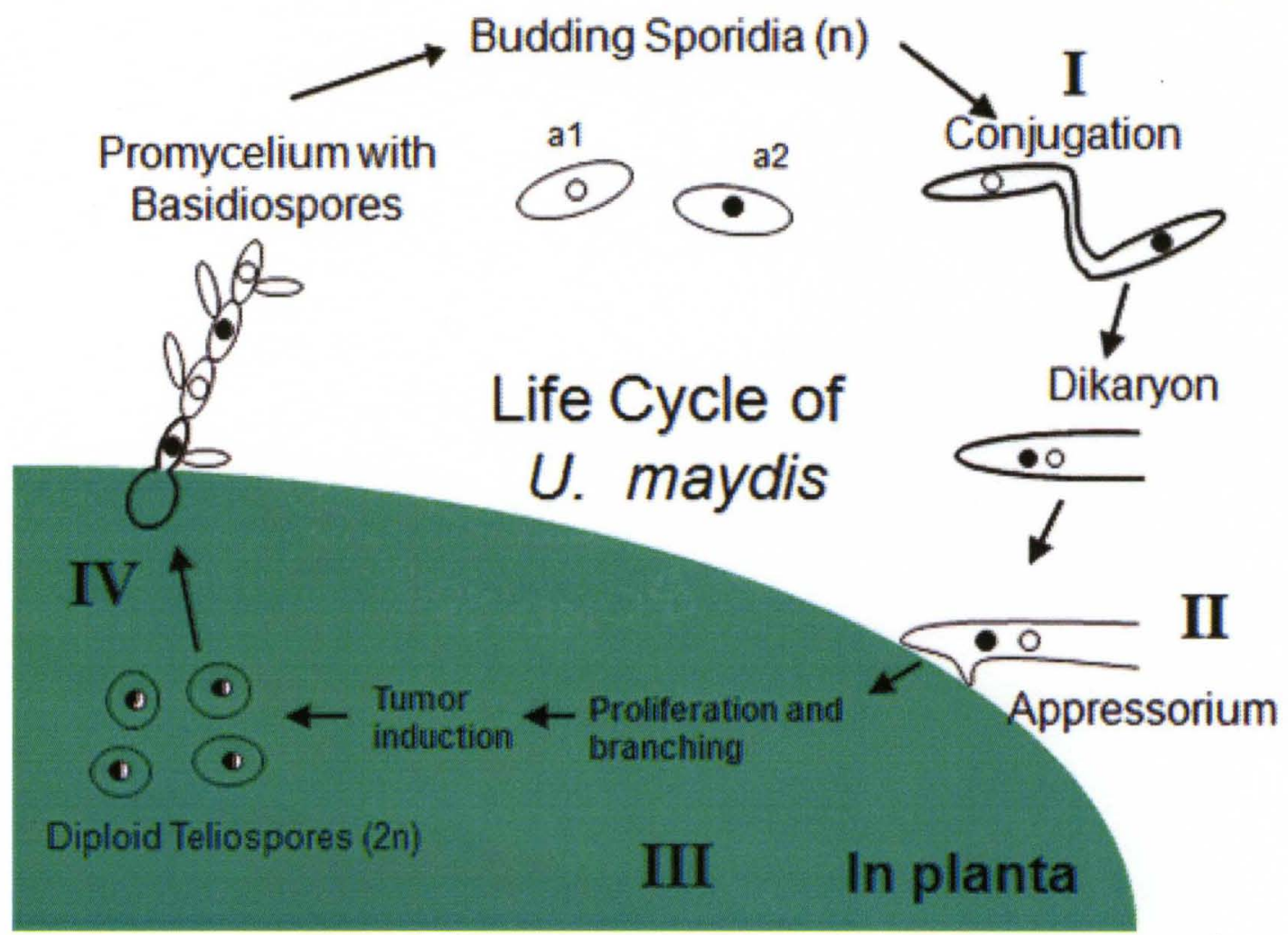

Figure 1: Representation of the life cycle of Ustilago maydis. Haploid sporidia can reproduce asexually by budding. In response to mating pheromone from a compatible partner they form conjugation (mating) filaments and fuse producing an infectious dikaryon which must penetrate the host plant to finish the life cycle. In the plant, hyphal filaments expand and then differentiate by nuclear fusion and produce diploid teliospores that mature and are released. The teliospores germinate, undergo meiosis, then mitosis and give rise to the next generation of haploid sporidia.

unicellular, uninucleate haploid form which is saprophytic, growing on non-living matter; a parasitic, dikaryotic hyphal form; and a diploid form (teliospore), which forms only in tumor-like galls produced in planta (for review see Banuett, 1995). Living freely in the environment as cigar-shaped, budding haploid sporidia, dimorphic transition to the dikaryotic filaments, and subsequent teliospore formation, is governed by two mating 
type loci, $a$ and $b$. Heterothallic in nature, mating requires cells with different alleles at both the $a$ locus and $b$ locus in order to complete the mating response. The $a$ locus, of which only two alleles are known ( $a 1$ and $a 2$ ), consists of at least two open reading frames (ORFs), mating pheromone a ( $m f a)$, which encodes a lipoprotein pheromone, and pheromone receptor a $(p r a)$, a seven-transmembrane-domain pheromone receptor (Banuett, 1995). Furthermore, the $a 2$ allele contains two additional ORFs, $\lg a 2$ and $r g a 2$, which encode small mitochondrial proteins. These proteins direct mitochondrial inheritance by selectively degrading $a l$-associated mtDNA, promoting $a 2 \mathrm{mtDNA}$ inheritance (Fedler, et al., 2009). The pheromone and pheromone receptor constitute the cell recognition system. The $a 2$ strain responds to basal levels of pheromone from a compatible $a 1$ strain causing an increase in $a 2$ pheromone expression. This induction leads to an increase in al pheromone expression promoting the growth of mating filaments (conjugation tubes) from both strains toward one another, ultimately resulting in cell fusion (Smith, et al., 2004).

Cell fusion of haploids gives rise to an infectious dikaryotic filament, where the cells fuse, but the nuclei do not. Cytoplasm in the dikaryotic filament is compressed to the apical tip by retraction septae, dividing the hyphal filament into compartments (Steinberg, et al., 1998). The resulting structure is then bound to completing its lifecycle through maize infection. However, the formation and stability of the dikaryon is dependent on an active heterologous $b$ locus that is responsible for self / non-self recognition. The $b$ locus encodes two divergently transcribed homeodomain proteins, $b E$ and $b W$, which act as a single genetic unit and constitute a heterodimeric transcription factor. The $b E / b W$ heterodimer likely functions as both a transcriptional activator and 
repressor, regulating the expression of genes necessary for pathogenesis in maize. Yet, only dimerization of a $b E$ and a $b W$ protein from different backgrounds will yield an active transcription factor and pathogenicity. Unlike the $a$ locus for which only two alleles have been identified, the current estimate on the number of $b$ alleles is over 25 , leading to hundreds of possible combinations of $b$ alleles and raising several questions about combinations and differences in virulence (Banuett, 1995; Bölker, 2001; Klosterman, et al., 2007). The dikaryon produced from mating of compatible partners then generates an appresorium to penetrate the plant. Unlike $M$. grisea (rice blast fungus) and Collectotrichum graminicola (pathogen of corn and wheat), which use turgor pressure to breach the surface of a leaf and gain entry to the plant, U. maydis secretes enzymes to cause local destruction of surface tissue in order to enter the plant (Kahmann and Kämper, 2004).

The dikaryon grows filamentously through the plant tissues, seemingly under the radar of the plant, not eliciting an immune response, and leads to the production of galls. Dikaryotic growth is difficult to replicate in vitro, indicating that plant cues may play a role in stabilizing the dikaryotic state of growth. The galls produced in planta result from plant cell proliferation promoted by the fungus. In the galls, the hyphal filaments undergo karyogamy and differentiate, giving rise to rounded diploid teliospores. These teliospores, which develop only in the plant, mature by producing melanin and a thick, rugged cell wall. The plant tissue making up the galls weakens, becoming fragile and ruptures releasing the darkened, soot-like teliospores into the wind. The tough cell wall of the teliospores protects the spores allowing them to remain dormant over an extended time frame of years. Nonetheless, in favorable conditions the teliospores germinate and 
undergo meiosis to form a promycelium. The promycelium consists of four haploid cells that can then undergo subsequent rounds of mitosis and give rise to the next generation of haploid sporidia. Mating between compatible haploid sporidia can occur in a laboratory setting, specifically on axenic, activated charcoal media. Mating two cells in this manner does not give rise to diploid teliospores, but provides a window into studying the regulation of cell morphogenesis and the mating response. Moreover, vegetative diploids, heterozygous at both mating loci, can be produced in vitro, allowing studies of post cell fusion events and processes (Banuett, 1995).

\section{REGULATING DIMORPHISM}

The dimorphic transition in fungi is a highly regulated process that incorporates several cell signaling pathways that are conserved among dimorphic fungi. Fungal cells need to coordinate the cell cycle, cytoskeletal elements, cell wall enzymes, and other cell components to complete the dimorphic transition. In addition, these various cell elements need to be localized to areas of active remodeling during the dimorphic transition. In nutrient rich conditions, $S$. cerevisiae normally displays isotropic growth producing round yeast-like cells that reproduce asexually by budding. These cells only briefly undergo a polar growth pattern during bud emergence, then the bud resorts to isotropic growth within the bud only (Madden and Snyder, 1998). However, in response to various signaling cues, $S$. cerevisiae can undergo a morphological transition producing polarized growth. Under conditions of low ammonium, yeast cells begin to elongate and, depending on the ploidy of the cells, promote invasive growth on solid media (haploid cells) or pseudohyphal growth (diploid cells). Additionally, pheromones can trigger polar growth as cells of opposite mating background respond to mating pheromone and 
arrest in G1, subsequently forming elongated mating projections or shmoo's (Madden and Snyder, 1998). U. maydis undergoes similar morphological transitions. Cells are polar in appearance and divide asexually by budding, but the buds never transition from a polar style of growth to isotropic growth as observed in S. cerevisiae. Additionally, both nutrient availability and mating pheromone promote filamentous growth in $U$. maydis. Under low ammonium conditions and when sensing mating pheromone from a compatible mating partner, cells can produce filaments (Banuett, 1995; Smith, et al., 2003). In addition, heterozygosity at the $b$ locus in haploids can lead to filamentation as well, though this is normally dependent on cell fusion on a suitable host (Brachmann, et al., 2001).

\section{THE CAMP DEPENDENT PROTEIN KINASE A PATHWAY}

At least two well studied and conserved signaling pathways regulate the morphogenic transition in $S$. cerevisiae. The first pathway, the cyclic AMP (cAMP) dependent Protein Kinase A (PKA) pathway, is composed of a series of enzymes that regulate cell responses to nutrient conditions by manipulating cAMP levels (Fig. 2, for review see Lengeler, et al., 2000). The PKA pathway comprises two core components, adenylate cyclase (AC) and the heterodimeric module, PKA. The PKA dimer consists of a regulatory subunit and a catalytic subunit and when the heterodimer is constituted, the regulatory subunit inhibits the catalytic subunit function. Activity of the heterodimer is influenced by intracellular cAMP levels, where binding of cAMP to the regulatory subunit causes its dissociation regulatory subunit from the catalytic subunit, freeing the catalytic subunit and allowing it to phosphorylate downstream targets. In $S$. cerevisiae, the activity of PKA promotes agar invasion and pseudohyphal differentiation. Three 

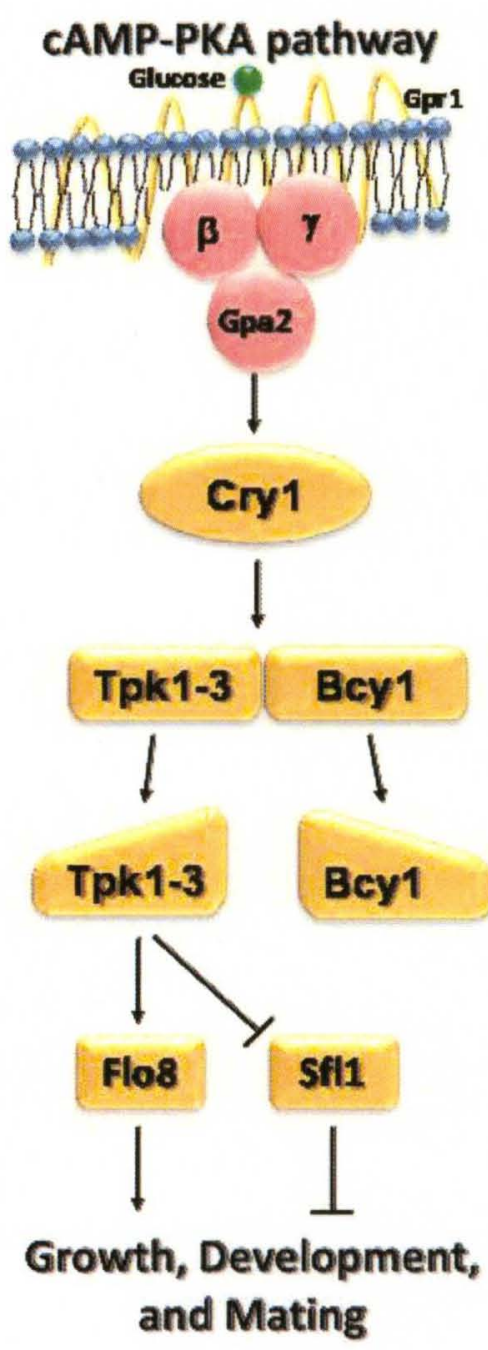

Figure 2: General diagram of the cAMP dependent PKA pathway in $S$. cerevisiae. This signaling pathway is important in nutrient sensing in fungi. In $U$. maydis it also plays a central role in pheromone-response and pathogenic development.

genes have been identified that encode catalytic subunits (tpkl, 2, and 3) and one gene encodes the regulatory subunit $(b c y l)$ (for review see Lengeler, et al., 2000).

In yeast, nutrient signals, specifically fermentable sugars, are perceived by a GPRC system. The GPRC, comprised of Gpr1, a seventransmembrane domain receptor and Gpa2, the associated $\mathrm{G} \alpha$ subunit of the heterotrimeric Gprotein, respond to glucose and ammonium levels to trigger a dramatic increase in cAMP levels (Lengeler, et al., 2000; Gagiano, et al., 2002). In addition, the ammonium transceptor, Mep2, triggers an increase in cAMP, though the mechanism is yet to be elucidated. Adenylate cyclase and phosphodiesterases regulate the intracellular levels of cAMP. Adenylate cyclase converts ATP to cAMP when activated by Gpa2, causing cAMP levels to increase. Subsequently, cAMP binds to the regulatory subunit of PKA, Bcy1, freeing the catalytic subunits, Tpk13. One group of downstream targets of Tpk1-3 is the phosphodiesterases, Pde1 and Pde2. Phosphodiesterases act by converting cAMP to various other phosphate forms. Pde1 is a low affinity phosphodiesterase, while Pde2 is a high affinity phosphodiesterase. In addition, Pde1 acts as a phosphoprotein and has a single PKA phosphorylation site 
indicating that it may act in a negative feedback loop serving to limit cAMP levels. Downstream of PKA, Tpk1-3 phosphorylate and activate the transcriptional activator Flo8, which activates several genes promoting pseudohyphal development, including $m u c 1$ and flo 11. Additionally, Tpk2 associates with the transcriptional repressor Sfl1 and prevents its function (Lengeler, et al., 2000; Gagiano, et al., 2002).

In $U$. maydis, mating and hyphal development are tightly linked and the cAMP dependent PKA pathway plays a major role in response to mating pheromone (for review see Klosterman, et al., 2007). Four $\mathrm{G} \alpha$ subunits have been identified in U. maydis, however, only Gpa3 is important in the mating response (Klosterman, et al., 2007). Similar to $S$. cerevisiae, the $U$. maydis genome contains only one gene that encodes the regulatory subunit of PKA $(u b c 1)$, but, different from yeast, only two genes encoding catalytic subunits of PKA are present ( $a d r l$ and $u k a l$ ), though $u k a l$ does not appear to play a role in mating, morphogenesis, and virulence (Gold, et al., 1994; Dürrenberger, et al., 1998). Activation of the adenylate cyclase Uac 1 by Gpa3 leads to increased cAMP levels. cAMP binding to Ubc1 frees the catalytic subunits, Adrl and Ukal, which leads to activation of the Pheromone-Responsive transcription Factor, Prfl (Kaffarnik, et al., 2003). The activation of Prf1 is necessary for the up-regulation of the $a$ and $b$ loci in the mating response. Adr1 also targets Crk1, an Ime2-like kinase that is required for proper mating and development of $U$. maydis. Through Adr1, the cAMP-PKA pathway negatively regulates $c r k l$ expression modulating mating and pathogenic development (Klosterman, et al., 2007).

As observed in $S$. cerevisiae, the cAMP-PKA pathway in $U$. maydis regulates the filamentous response to low ammonium conditions. Whereas increased cAMP levels 
lead to pseudohyphal development in S. cerevisiae, in U. maydis the opposite is true. Increased cAMP levels leads to an active catalytic subunit of PKA, but here it promotes budding instead of the filamentous response. The Ump2 transceptor, the Mep2 homologue in $U$. maydis, promotes the filamentous response by decreasing intracellular cAMP levels. However, the mechanism of cAMP reductions by Ump2 is presently unknown. Interestingly, though intracellular levels of cAMP are opposite between $S$. cerevisiae and $U$. maydis under nitrogen starvation conditions, the overall outcome of this condition is the same. Both $S$. cerevisiae and $U$. maydis transition to filamentous growth through the cAMP-PKA pathway when starved for nitrogen (Smith, et al., 2003).

\section{THE MITOGEN-ACTIVATED PROTEIN KINASE (MAPK) PATHWAY}

While the cAMP-PKA pathway regulates the filamentous response in both $S$. cerevisiae and $U$. maydis, it is one of the two highly conserved and studied pathways involved in the morphogenic transition in both fungi. The cAMP-PKA pathway is primarily involved in nutrient sensing, while the Mitogen-Activated Protein Kinase (MAPK) pathway plays a role in both mating and filamentous growth. Here I will focus on the mating pheromone response pathway (for review see Bardwell, 2005). The MAPK pathway, also known as the Extracellular signal-Regulated Kinase (ERK) pathway, consists of a core module of three phosphoprotein kinases that relay an extracellular signal to the nucleus (Fig. 3). The pathway is a serial cascade of phosphorylation events triggered by, and targeting three protein kinases, a MAPK kinase kinase (MAPKKK), also known as a MAPK / ERK kinase kinase (MEKK), a MAPK kinase (MAPKK / MEKK), and a MAP kinase (MAPK or ERK), which in turn 


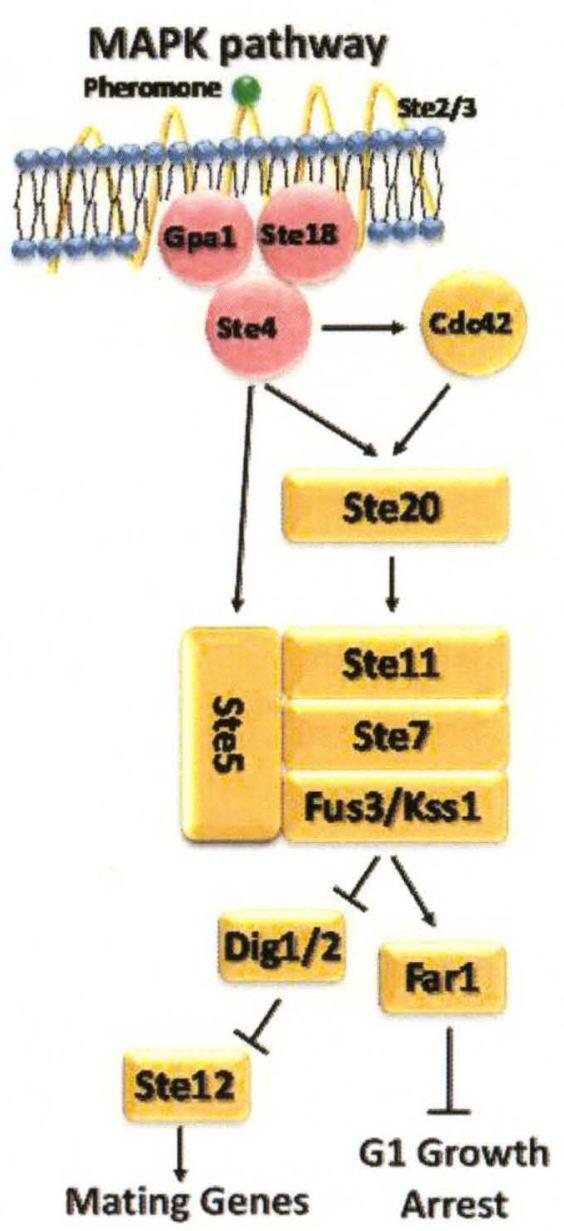

Figure 3: Diagram of the $S$. cerevisiae MAPK pathway. This pathway plays a role in both pheromone response and pseudohyphal differentiation. The signal is transmitted via activation of a heterotrimeric G-protein which activates a series of phosphorylation events that proceed through the three component module that comprises the MAPK pathway. This ultimately leads to transcriptional regulation of genes involved in either mating or phosphorylates one or more targeted substrates, some of which are transcription factors. In $S$. cerevisiae, Ste11 is the MAPKKK / MEKK, while Ste7 is the MAPKK / MEK and both Fus3 and Kss1 are MAPKs / ERKs. However, the pathway is more complex, consisting of several more components both upstream and downstream of the core module.

Mating pathway signaling begins with a mating pheromone binding to the seventransmembrane domain receptor, Ste2 (Ste3 for opposite mating pheromone). Upon the pheromone binding to the receptor, the $\mathrm{G} \beta \gamma$ heterodimer complex of Ste18 and Ste4, respectively, binds both $\mathrm{p} 21$-activated kinase (PAK) Ste20 and the scaffold and adaptor Ste5. The Ste18 / Ste4 complex is anchored to the plasma membrane by covalent linkage of Ste18 to the lipid bi-layer. Ste5 binds to the Ste18 /

Ste4 complex and to Ste11 bringing Ste11 to the lipid bi-layer. The Ste18 / Ste4 complex also binds Ste20 bringing it to the plasma membrane in the vicinity of Ste 5 / Ste11, though some studies suggest that a cryptic membrane binding motif rich with basic residues (BR) localizes Ste20 to the plasma membrane (Takahashi and Pryciak, 2007). In 
addition, Ste 20 may be brought to the plasma membrane by its association to the monomeric Rho-like GTPase, Cdc42. Cdc42 interacts with Ste20 though the specific CRIB (Cdc42 / Rac Interactive Binding) domain and this interaction activates Ste20 function. Cdc42 binding alleviates the auto-inhibition of the CRIB domain and allows Ste18 / Ste4-mediated signaling (Lamson, et al., 2002). The proximity of Ste20 and Ste1 1 allows Ste20 to phosphorylate Ste11 and activate it. Additional targets of the Ste18 / Ste4 complex are Far1 and Cdc24. Cdc24 is the Guanine nucleotide Exchange Factor (GEF) for Cdc42 and functions by catalyzing the exchange of GDP with GTP, subsequently activating Cdc42, allowing it to bind to Ste20 and other targets. Farl acts as an adaptor for Cdc24 and Cdc42. Also, Farl acts a downstream target of the MAPK pathway, promoting cell cycle arrest in G1.

Once activated, Ste11 begins the serial cascade of phosphorylation events that pass through Ste7 (the MAPKK / MEK) and then Fus3 and Kss1 (the MAPK / ERK), all organized and isolated by Ste5. Both Fus3 and Kss1 are known to mediate the output of the pheromone response pathway. They regulate the activity of the transcription factor Ste12 by directly phosphorylating Ste12 as well as the Ste12 repressors Dig1 and Dig2. These phosphorylation events lead to Ste 12 activity and expression of the mating genes. In addition, Fus 3 also targets and activates Far1, promoting a second role for Far1 beyond tethering Cdc24 and Cdc42. Upon activation, Farl targets the cyclin dependent kinase, Cdc28, and inhibits its function leading to cell cycle arrest at G1 (Bardwell, 2005).

The MAPK pathway also plays a role in the filamentous response to environmental conditions in addition to the mating response (for review see Chen and Thorner, 2007). Though several different components constitute the filamentous 
response pathway, the core components of Cdc42, Ste20, Ste11, Ste7, Kss1, and Ste12 are still utilized. Several different transmembrane receptors are necessary for the filamentous response though the MAPK pathway, including Mep2. Signal transduction from membrane to $\mathrm{Cdc} 42$ is not clearly understood, but it does require the small monomeric G-protein, Ras2, and the 14-3-3 homologues, Bmh1 and Bmh2. The signal follows an identical path as observed in the mating response pathway, with the exception that only Kss1 is activated, not Fus3 (mating response pathway only). Ste12 up-regulates mating gene expression, but in the filamentous response pathway, Ste12 associates with another transcription factor Tec1p to promote expression of genes involved in the filamentous response.

A similar MAPK pathway exists in $U$. maydis and is responsible for the pheromone response (for review see Klosterman, et al., 2007). Unlike S. cerevisiae, several players in this pathway are either not known or poorly understood. Signaling begins with pheromone (Mfa1/2) binding to the pheromone receptor (Pra1/2). However, the connection to the next member in the pathway, Ras2, remains unclear. Through domain examination, Ras2 potentially interacts with the MAPKKK / MEKK Ubc4 / Kpp4 and the potential Ste50-like scaffold, Ubc2. Once activated Ubc4 / Kpp4 phosphorylates Ubc5 / Fuz7 (MAPKK / MEK), which then targets Ubc3 / Kpp2 (MAPK / ERK). Downstream of Ubc3 / Kpp2 are the two known MAPK targets Prf1 and Crk1, both of which lead to induction of the mating loci. In addition, a second MAPK / ERK, Kpp6 leads to plant penetration. Other proteins known to play a role in the mating response are the PAK-like kinases, Smul and Cla4. Both kinases display differential mating-type dependent effects, with deleting smul attenuating pheromone expression in 
the $a 2$ mating-type background, whereas deleting cla 4 reduces mating efficiency in the al mating-type background (Smith, et al., 2004; Leveleki, et al., 2004). Beyond the mating response, the MAPK pathway has also been implicated in regulating filamentous growth, though its involvement is not clear.

\section{CROSSTALK BETWEEN THE MAPK AND cAMP-PKA PATHWAY}

Studies exploring both the cAMP-PKA and MAPK pathways have revealed several points of cross talk between the two pathways. Lengeler et al. (2000) posited three points of intersection between the cAMP-PKA and MAPK pathways in $S$. cerevisiae. Upstream of both pathways, the G-protein Ras2 activates both pathways, though the exact mechanism and points of control have yet to be elucidated.

Downstream, activity of the two pathways converge on the expression of flo11, a cell surface flocculin that promotes cell to cell adhesion, necessary for pseudohyphal development and agar invasion (Palecek, et al., 2000). Last, high levels of cAMP inhibit MAPK activity, leading to the idea that the cAMP-PKA pathway antagonizes the MAPK pathway at certain points, while at other points the two pathways may work synergistically promoting pseudohyphal development and agar invasion (Lengeler, et al., 2000).

A similar form of crosstalk has also been observed in U. maydis, in which several genes act to suppress the constitutively filamentous phenotype of strains lacking the adenylate cyclase, $\mathrm{uacl}$ (Fig. 4). The suppressor genes are known as Ustilago Bypass of Cyclase (Ubc) and rescue the filamentous phenotype observed in the $u a c l$ deletion strain (Klosterman, et al., 2007). Five genes were isolated that were able to bypass the $\Delta u a c 1$ phenotype, three of which (Ubc3-5) were the components of the MAPK module. In 


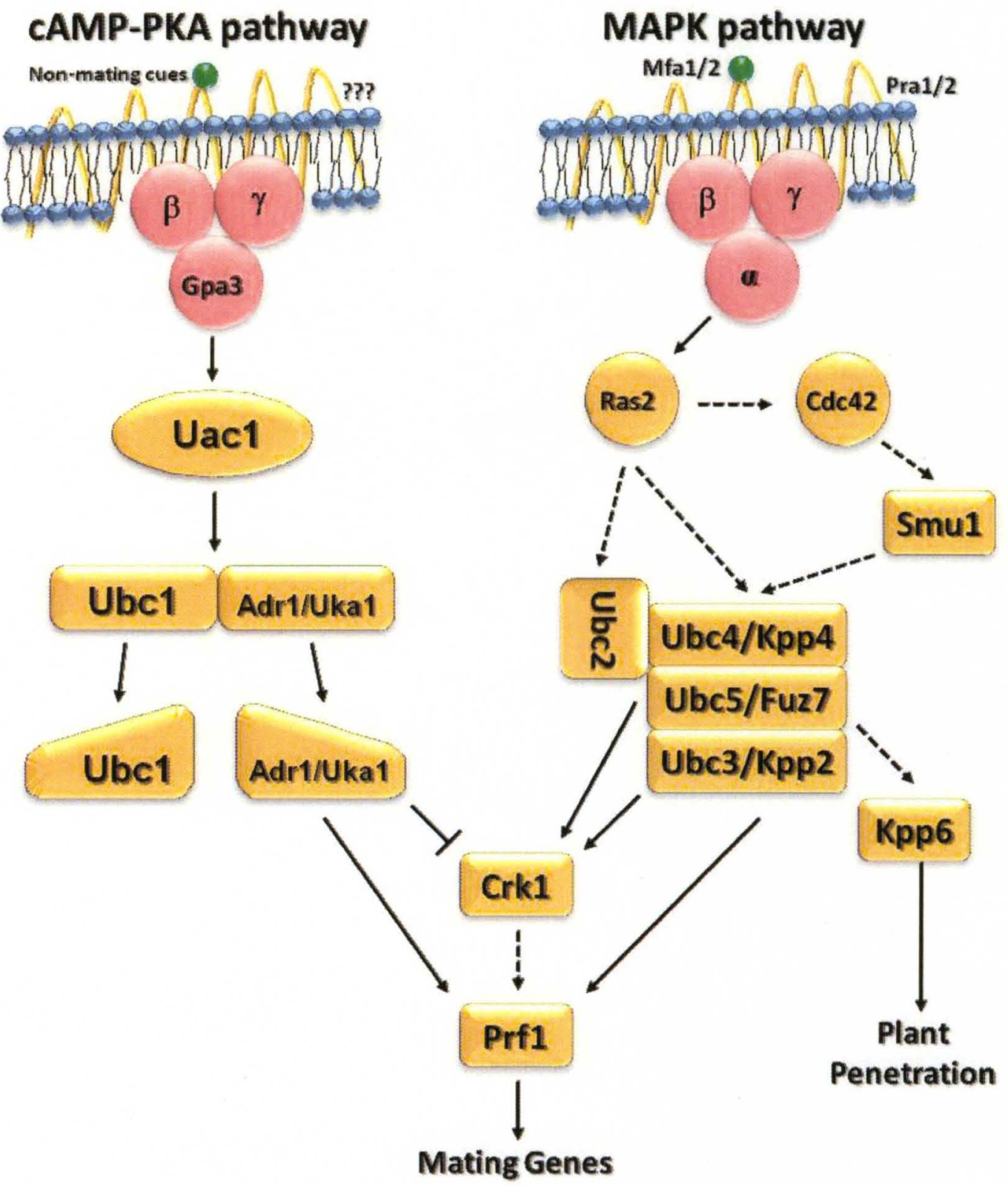

Figure 4: Representation of cAMP-PKA and MAPK pathway from $\boldsymbol{U}$. maydis. Both pathways converge on two different targets, Prfl and Crk1 to modulate mating, morphogenesis, and pathogenicity. Solid lines represent and established interaction, either genetic or physical, Dashed lines are hypothetical / potential interactions. See text for additional information.

addition, Ubc1 is the regulatory subunit of PKA, and Ubc2 is the Ste5-like adaptor protein (Klosterman, et al., 2007). Moreover, the transcription factor, Prf1, is differentially phosphorylated by both Adr1 (catalytic subunit of PKA) and Ubc3 / Kpp2 (MAPK / ERK) leading to expression of either the $a$ mating-type genes (Adr1 dependent) 
or expression of the $b$ mating-type genes (Adr1 and Ubc3 / Kpp2 dependent). Further, both the cAMP-PKA and MAPK pathway differentially converge on Crk1. cAMP-PKA pathway negatively regulates $c r k 1$ expression, while the MAPK pathway activates Crk1 activity (Klosterman, et al., 2007).

\section{BEYOND THE MAPK PATHWAY}

Several additional proteins are involved in the modulation of mating signaling through the MAPK pathway. Many of these proteins also play roles in processes outside of the MAPK pathway. Bem1p is one such protein. It acts as an adaptor and scaffold bringing the Cdc24 / Cdc42 complex in close proximity to Ste5 and the MAPK module, as well as Ste20. Cdc24 localizes to the plasma membrane through pleckstrin homology $(\mathrm{PH})$ and interaction with the adaptor, Far1. Through direct binding, Bem1 $\mathrm{p}$ also interacts with Ste5 and Ste20, and this association brings Ste20 and the MAPK module together with the Cdc24-Cdc42 complex, allowing Cdc42 to activate Ste20 function on Ste11 (Lyons, et al., 1996; Winters and Pryciak, 2005; Chen and Thorner, 2007).

The activation of Ste11 is just one role Ste20 plays in yeast. As previously mentioned, the PAK-like kinase Ste20 plays an extremely important role in both the MAPK pathway, regulating both the mating response and the filamentous response to environmental conditions, and cell morphogenesis and cytokinesis. The PAKs comprise a large family of highly conserved serine/threonine protein kinases. Ste20 is a downstream target of $\mathrm{Cdc} 42$ in both the mating-responsive MAPK pathway and in actin organization in polarized cell growth (Eby, et al., 1998; Holly and Blumer, 1999; Lengeler, et al., 2000). Ste20 also plays a role in the filamentous-responsive MAPK pathway, and mitotic exit (Lengeler, et al., 2000; Höfken and Schiebel, 2002; Chen and 
Thorner, 2007). Evidence suggests that Ste20 is required throughout the cell cycle regulating actin and cell polarity (Holly and Blumer, 1999). In addition, Ste20 appears to be regulated by the cell cycle machinery. Cdc28 targets Ste20, altering its downstream outcome. When activated, Ste20 targets the MAPK pathway and the mating response. However, under the activity of pheromone, Farl triggers a G1 arrest by targeting Cdc28, thereby allowing Ste20 function to proceed through the mating response pathway. In the absence of pheromone, Far1 does not inactivate Cdc28, allowing Cdc28 to target Ste20; this change shifts its function from the mating response pathway to the filamentous response pathway (Oehlen and Cross, 1998; Wu, et al., 1998).

Overall it appears that the key to the differences between the mating-responsive and filamentous-responsive MAPK pathway is the activation of Far1 by presence of mating pheromone. Furthermore, Ste20 is not the only downstream target of the Cdc24 / Cdc42 complex. Cla4, a second PAK-like kinase, is a downstream target of Cdc42 involved in septin formation and cell polarity (Versele and Thorner, 2004). Normal septin formation and Cla4 activity promotes the G2-M transition through the protein kinase Swe1, though early in the cell cycle Cla4 is required for normal actin polarity (Holly and Blumer, 1999; Versele and Thorner, 2005). Cla4 also may play a role in the pheromone response in yeast, potentially through interacting with Cdc42 allowing Ste20 function to promote the mating response. Failure of $\mathrm{Cdc} 42$ and Cla4 to interact may reduce MAPK activity by attenuating Ste20 activity and preventing the G1 arrest required for mating, leading to Cla4 and Ste20 antagonism in the mating response (Benton, et al., 1997; Heinrich, et al., 2007). 
Several points of overlap in function between Ste20 and Cla4 have been identified. Neither gene product, by itself, is essential, though absence of one gene or the other creates cell defects in mating, pseudohyphal development and agar invasion, proper bud formation, or cytokinesis (Cvrčková, et al., 1995; Benton, et al., 1997; Eby, et al., 1998; Holly and Blumer, 1999; Lengeler et al., 2000; Heinrich et al., 2007). Although deletion of one gene leads to specific associated non-lethal phenotypes, deletion of both ste 20 and cla4 is lethal and the cells arrest after forming one to two elongated buds (Cvrčková, et al., 1995). Both Ste20 and Cla4 are targets of GTP-bound Cdc42 and play roles in actin polarization, pheromone response, vacuole inheritance, and cytokinesis (Cvrčková, et al., 1995; Benton, et al., 1997; Eby, et al., 1998; Holly and Blumer, 1999; Lengeler et al., 2000; Heinrich et al., 2007). The shared activities of Ste20 and Cla4 may be due to similarity in their structure, differing by only one domain. Cla4 contains a $\mathrm{PH}$ domain which facilitates the interaction between Cla4 and the plasma membrane. Ste20 does not contain a PH domain, but is still able to interact with the plasma membrane through the BR region in the $\mathrm{N}$ terminus (Takahashi and Pryciak, 2007). Work by Keniry and Sprague (2003) approached the question of overlap between Ste20 and Cla4 by creating chimeras of the two proteins. Their work identified a single amino acid substitution that imparted many Ste20-specific functions to Cla4 without ablating any Cla4-specific functions. In addition, they recognized that several functions of both Ste20 and $\mathrm{Cla} 4$ require more than one region of the protein, thereby complicating the ability to fully separate the functions of either.

A similar type of overlap between Ste20 and Cla4 homologues has been observed in Schizosaccharomyces pombe, C. neoformans, and U. maydis (Yang, et al., 1998; 
Wang, et al., 2002; Leveleki, et al., 2004; Nichols, et al., 2004; Smith, et al., 2004). In these three cases, the extent of overlapping functions was observed to be distinct between the Ste20 and Cla4 homologues. S. pombe provides an interesting picture where in the Shk1 (Ste20 homologue) is essential for viability, mating, cytoskeletal regulation, cell cycle regulation, and response to hyperosmotic conditions, while Shk2 (Cla4 homologue) is dispensable for growth and appears to be redundant to Shk1 (Marcus, et al., 1995; Gilbreth, et al., 1998; Yang, et al., 1998; Bao, et al., 2001; Qyang, et al., 2002). This differs from S. cerevisiae in that both Ste20 and Cla4 overlap in function, yet they are separable in many of these functions. Similar to the yeast model, Ste20a/ $\alpha$ (Cla4 homologue) and Pak1 (Ste20 homologue) of C. neoformans display overlapping functions in mating, differentiation, cytokinesis and virulence; however, the roles of the two PAKs can be delineated both functionally and temporally (Wang, et al., 2002; Nichols, et al., 2004). Pak1 plays a role in the cell fusion, while Ste20a/a is essential for maintaining polarity during dikaryotic growth. $U$. maydis displays a unique mating-type specific overlap between Smul (Ste20 homologue) and Cla4 in mating and virulence, where both Smu1 and Cla4 have roles in mating and pathogenic development (Leveleki, et al., 2004; Smith, et al., 2004). More specifically, Smul is necessary for up-regulation of pheromone expression but the defect is more severe when $s m u 1$ is deleted in the $a 2$ mating-type background. On the other hand, Cla4 is dispensable for pheromone expression, but necessary for cytokinesis and proper filament formation, yet when lacking cla4 the defect is greater in the al mating-type background.

Both Ste20 and Cla4 homologues are involved in a myriad of functions that need to be modulated to respond to a given set of conditions. Several proteins modulate Ste20 
function in S. cerevisiae, the Ste18 / Ste4 complex, Cdc42, Bem1, Ste5, Bmh1, Bmh2, and $\mathrm{Cdc} 28$ activate and modulate Ste 20 activity between the mating responsive and filamentous-responsive MAPK pathway (Roberts, et al., 1997; Cabib, et al., 1998; Bardwell, 2005). Another protein demonstrated to modulate Ste20 activity is the putative protein-arginine methyltransferase (PRMT), Hsl7 (Fujita, et al., 1999). PRMTs facilitate methylation on arginine residues and play roles in signal transduction, RNA processing, and transcriptional regulation $(\mathrm{Ma}, 2000)$. Hsl7 has been shown to have methylation activity in vitro, though no methylation activity has been observed in vivo (Lee, et al., 2000; Miranda, et al, 2006). Hsl7 was initially identified through a synthetic lethal screen examining second site mutations that were sensitive to the absence of the $\mathrm{N}$ terminus of histone H3 (Ma, et al., 1996). Several studies have demonstrated that Hs17 regulates cell cycle by acting as an adaptor between Hsl1 and Swe1, targeting Swe1 for degradation and thus promoting G2-M transition (Ma, et al., 1996; Shulewitz, et al., 1999; Cid, et al., 2001; La Valle and Wittenberg, 2001; Asano, et al., 2005). Fujita, et al. (1999) demonstrated that Hsl7 also interacts both in vitro and in vivo with Ste20, competing with $\mathrm{Cdc} 42$, and negatively regulates the filamentous response to low ammonium conditions. However, evidence from Shulewitz, et al. (1999) indicates that Hsl7 influence on the filamentous response may be independent of Ste20, instead promoting cell elongation through a Swe1-specific G2-M delay. Hsl7 homologues in both $S$. pombe and Xenopus laevis also regulate cell cycle in a Wee1 (Swe1 homologue) dependent manner (Gilbreth, et al., 1996 and 1998; Yamada, et al., 2004). Skb1 (the Hsl7 homologue in S. pombe) interacts with the S. pombe Ste20 homologue, Shk1, and regulates cell cycle and the response to hyperosmotic conditions (Gilbreth, et al., 1998; 
Bao, et al., 2001). Under these hyperosmotic conditions, Skb1 displays an increase in its methyltransferase activity (Bao, et al., 2001). Also known as Jbp1 and PRMT5, the human Hs17 homologue interacts with the non-receptor tyrosine kinase, Jak2, and exhibits methyltransferase activity in vivo (Pollack, et al., 1999; Rho, et al., 2001). The methyltransferase activity has been linked to histone $\mathrm{H} 3$ and regulates both cell cycle progression at G1-S and tumor suppression by targeting the tumor suppressors, ST7 and $\mathrm{p} 53$, through the translation initiation factor eIF4E (Pal, et al., 2004; Scoumanne, et al., 2009).

\section{INITIAL RESEARCH INTEREST OF THIS DISSERTATION}

The Perlin lab has been and continues to explore several members of the MAPK and cAMP-PKA signaling pathways in U. maydis. These pathways, as described above, are critical in regulating the mating response, morphogenesis, and virulence. Both Hsl7 and Ste 20 homologues are important components in regulating the activity of the MAPK pathway. In S. cerevisiae, Ste20 plays a role in both the mating and filamentous response MAPK pathways and several different proteins differentiate and direct Ste20 activity towards one of the two possible outcomes of the MAPK pathways (Lengeler et al., 2000; Bardwell, 2005). Hsl7 is one of these proteins, acting as a negative regulator of Ste20 in the filamentous response pathway (Fujita, et al., 1999).

My interest in Hs17 is based on the identification and characterization of Smul, a Ste20 homologue in U. maydis (Smith, et al., 2004). Smu1 is required for proper mating and pathogenicity. Specifically, Smu1 plays a role in the up-regulation of pheromone ( $m f a$ ) expression. The presence of a putative Hsl7 in $U$. maydis encouraged me to question whether the product of $h s l 7$ interacts with Smul, as observed in S. cerevisiae, 
and what role it might play in the mating response and pathogenicity. My initial observations led me to explore the role of $\mathrm{Hsl} 7$ in the filamentous response to low ammonium conditions. In addition, it triggered questions of the regulation of filament formation through the Rho-like G protein, Rac1, and by Cla4. In the remainder of this dissertation, I present data that suggest that both Smul, with Hs17, and Cla4 play a role in the cell polarity filament formation and cell wall localization under conditions of low ammonium. 


\section{CHAPTER II}

\section{THE METHYLTRANSFERASE HSL7 AND THE PAK KINASE SMU1 INTERACTIONS AFFECT CELL SIZE, THE FILAMENTOUS RESPONSE, AND PATHOGENICITY INTRODUCTION}

p21-activated protein kinases (PAKs) comprise a large, highly conserved family of serine/threonine protein kinases, involved in a myriad of cellular functions including cell cycle regulation, cytoskeletal organization, mating responses, cell polarity, cell morphogenesis, cell separation, and response to environmental conditions (Roberts and Fink, 1994; Cvrčková, et al., 1995; Marcus, et al., 1995; Gilbreth, et al., 1996 and 1998; Leberer, et al., 1997; Eby, et al., 1998; Holly and Blumer, 1999; Fujita, et al., 1999; Bao, et al., 2001; Höfken and Schiebel, 2002; Qyang, et al., 2002; Wiley et al., 2003; Leveleki, et al., 2004; Smith, et al., 2004; Heinrich, et al., 2007; Böhmer, et al., 2008). Homologues of the PAK family are known to activate the conserved, three component module, mitogen activated protein kinase (MAPK) pathway (Lengeler et al., 2000; García-Pedrajas, et al., 2008). These pathways are conserved from fungi to humans and play a role in several fungal processes, including mating, cell morphology, growth under conditions of high osmolarity, and filamentous growth. This pathway consists of a serial cascade of phosphorylation events triggered by, and targeting three protein kinases, a MAPK kinase kinase (MAPKKK), a MAPK kinase (MAPKK), and a MAP kinase 
(MAPK), which in turn phosphorylates one or more targeted substrates, some of which are transcription factors. In Saccharomyces cerevisiae, both mating and invasive growth / pseudohyphal differentiation depend on multiple elements of a single MAPK cascade (Roberts and Fink, 1994). In Schizosaccharomyces pombe, the MAPK pathway is involved in the mating response and subsequent meiosis and sporulation. Several components are shared between the two subsets of the pathways including the PAK, MAPKKK and the MAPKK (Neiman et al., 1993; Marcus, et al., 1995; García-Pedrajas, et al., 2008). Discriminating between various inputs and the eventual desired outputs is dependent on a series of scaffolding and adaptor proteins. In addition, upstream activators and co-activators dissect eventual outputs though spatial regulation of components of this pathway. Upstream of the PAK's, and subsequently the MAPK pathway, is a series of conserved Rho/Rac-like GTP-binding proteins (GTPases) which bind and regulate the localization and activity of the PAK's (Marcus, et al., 1995; GarcíaPedrajas, et al., 2008).

One member of the Rho/Rac family of GTPases is $\mathrm{Cdc} 42$. Cdc42 plays a role in regulating cell proliferation, polarity, and differentiation in many eukaryotes. In $S$. cerevisiae, $\mathrm{Cdc} 42$ is required for cell polarity, cytokinesis, regulating invasive and pseudohyphal development, and mating of haploid cells (Etienne-Manneville, 2004; Lengeler et al., 2000). Cdc42 specificity and activity is regulated by cycling between GTP (active) and GDP (inactive) states via two additional groups of proteins, guanine exchange factors (GEFs) and GTPase activating proteins (GAPs). One such GEF is Cdc24, which localizes Cdc42 to the bud neck allowing for proper formation of bud, cytoskeletal reorganization and cytokinesis. A multiprotein complex comprised of 
several proteins including, $\mathrm{Cdc} 42$ and Scd1 (Cdc24 homologue), plays a similar roles in S. pombe (Chang et al., 1999).

Of potential downstream effectors of $\mathrm{Cdc} 42$ and $\mathrm{Cdc} 24$, the PAKs have been extensively studied (see above). Ste20 (Shk1) and Cla4 (Shk2) are two PAK's first identified and well characterized in S. cerevisiae and S. pombe, and functions of the two are required in several key aspects of cell development. In yeast simultaneous deletion of both ste20 and cla4 is lethal, indicating the two PAK homologues share at least one essential function (Cvrčková, et al., 1995). In addition, Ste20 and Cla4 play roles in many distinct processes. Ste20 is involved in pheromone response pathway, haploid invasive growth pathway, and osmosensing high-osmolarity glycerol (HOG) pathway, while Cla4 regulates septin function and polarized growth as well as cytokinesis. Data have linked Ste20 to cell polarity and cytokinesis and Cla4 to the mating response and both to actin organization (Cvrčková, et al., 1995; Benton, et al., 1997; Eby, et al., 1998; Holly and Blumer, 1999; Lengeler et al., 2000; Heinrich et al., 2007). In S. pombe, Shk1 is essential for viability and is required for several cell functions, including mating, cytoskeletal regulation, cell cycle regulation, and response to hyperosmotic conditions, while Shk2, dispensable for growth, appears to be redundant to Shk1in many functions (Marcus, et al., 1995; Gilbreth, et al., 1998; Yang, et al., 1998; Bao, et al., 2001; Qyang, et al., 2002).

In U. maydis, several Rho-like GTPases have been identified, Cdc42, Rac1, Rho1, and Rho3, of which Cdc42, Rac1, and Rho1 regulate cell separation, hyphal development, and cell polarity, respectively (Weinzierl, et al., 2002; Mahlert, et al., 2006; Böhmer, et al., 2008; Pham et al., 2009). In addition, Cdc42, Raclp, and Rhol have 
been demonstrated to interact in vitro with two known PAK's from U. maydis, Cla4 and Smu1 (Leveleki, et al., 2004; Pham, et al., 2009). In vivo analysis has identified Cla4 to be an effector of Rac1, regulating cell polarity (Leveleki, et al., 2004). A Cdc24 homologue was identified in $U$. maydis, however Cdc24 is the preferred GEF of Rac1, not Cdc42 (Castillo-Lluva, et al., 2007; Alvarez-Tabarés and Pérez-Martín, 2008). A second GEF, Don1, so named due to the mutant colony morphology resembling donuts with a hollow, empty center, was identified as the activator of $\mathrm{Cdc} 42$, regulating formation of the second septum between mother and daughter cells. Moreover, a third kinase, Don3, belongs to the germinal centre kinase subfamily of PAK kinases, and is involved in the formation of the second septum between mother and daughter cells in cell separation (Weinzierl et al., 2002). Initially, Don3 was thought to be the effector of Cdc42, though further analysis indicated that the two act independently in the formation of the second septum (Böhmer, et al., 2008). Adonl and $\Delta d o n 3$ mutants are defective in cell separation. In addition, $\Delta c d c 42$ mutant strains exhibit the same phenotype(Weinzierl et al., 2002).

Hs17 homologues are known interacters of the PAKs and are conserved from fungi to humans (Ma, et al., 1996; Gilbreth, et al., 1996; Pollack, et al., 1999; Fujita et al., 1999). Hsl7 homologues are putative protein-arginine methyltransferases and are identified by a single methyltransferase domain (Ma, 2000). Hsl7 homologues are involved in a myriad of cellular functions ranging from cell cycle regulation, cell morphogenesis, and response to several environmental conditions (Ma, et al., 1996; Gilbreth, et al., 1996 and 1998; Pollack, et al., 1999; Fujita, et al., 1999; Bao, et al., 2001; Yamada, et al., 2004). Hsl7 from yeast acts as a mitotic inducer by promoting 
targeted Swe1 degradation and as a negative regulator of Ste20 in the filamentous response pathway, by acting as a competitive inhibitor of the Ste 20 activator Cdc 42 (Fujita et al., 1999; La Valle and Wittenberg, 2001; Asano, et al., 2005). Conversely Skb1, the Hsl7 homologue present in $S$. pombe, acts, in concert with Shk1, as a mitotic inhibitor in a Weel dependent fashion (Gilbreth, et al., 1998). The study presented here describes the isolation and characterization of an $\mathrm{Hsl} 7$ homologue from $U$. maydis and its negative interaction, and subsequent roles, with Smul. I hypothesized that Hsl7 would act as a negative regulator of Smul in the control of the filamentous response to low ammonium conditions, and potentially the mating response and virulence. Here I show that disruption of $h s l 7$ leads to increases in the filamentous response to low ammonium conditions, while no defects in the mating response and pathogenicity were observed. Concomitant overexpression of smul leads to an exacerbation of the filamentous response, as well as cell separation defects.

\section{MATERIALS AND METHODS}

Strains and Growth Conditions. Ustilago maydis strains utilized in this study are listed in Table 1. Saccharomyces cerevisiae strains AH109 (genotype: MATa, trpl901, leu2-3, 112, ura3-52, his3-200 gal4A, gal804, LYS2::GAL1 UAS-GAL1 $1_{T A T A}$ HIS3, GAL2 $2_{U A S^{-}} G A L 2_{T A T A^{-}} A D E 2, U R A 3:: M E L 1_{U A S^{-}} M E L 1_{T A T A^{-}}$lacZ) and Y187 (genotype: MATa, trp1-901, leu2-3, 112, ura3-52, his3-200 gal4A, met', gal804, URA3::GAL1 UAS$G A L 1_{T A T A}-$ lacZ) were obtained from Clonetech (Mountain View, CA) and Dr. S. Ellis (University of Louisville) and used for yeast two-hybrid experiments. Saccharomyces cerevisiae strains used for complementation, MJY102 (MATa, ADE2, can1-100, his311,15, leu2-3,112, LYS2, trp1-1, ura3-1, hsl7-420::HIS3) and MJY110 (MATa, ADE2, 
TABLE 1. $U$. maydis strains and plasmids used in this study.

\begin{tabular}{|c|c|c|}
\hline Strain / Plasmid & Genotype & Reference \\
\hline \multicolumn{3}{|l|}{ Fungal Strains ${ }^{A}$} \\
\hline FB1 WT & $a 1 b 1$ & Banuett, et al, 1989 \\
\hline$\Delta h s l 7$ al & 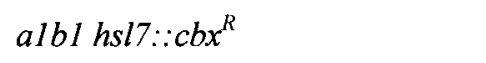 & This Study \\
\hline$\Delta_{810-2832} h s l 7$ al & albl hsl7::hyg ${ }^{R}$ & This Study \\
\hline$h s l 7^{\text {Oref }} a l$ & $a l b 1 P_{\text {otef }} h s l 7, c b x^{R}$ & This Study \\
\hline$\Delta s m u l$ al & $a 1 b 1 \operatorname{smu} 1:: c b x^{R}$ & This Study \\
\hline smul ${ }^{\text {Otef }}$ al & $a 1 b 1 P_{\text {otef }}$ smu1, $c b x^{R}$ & This Study \\
\hline$\Delta_{810-2832} h s l 7 \Delta s m u l$ al & alb1 hsl7::hyg ${ }^{R}$, smul $:: c b x^{R}$ & This Study \\
\hline$\Delta_{810-2832} h s l 7 s m u l^{O t e f}$ al & $a l b 1$ hsl7::hyg ${ }^{R}, P_{\text {otef }}-$ smul, $c b x^{R}$ & This Study \\
\hline FB2 WT & $a 2 b 2$ & Banuett, et al, 1989 \\
\hline$\Delta h s l 7$ a2 & $a 2 b 2 h s l 7:: c b x^{R}$ & This Study \\
\hline$\Delta_{810-2832} h s l 7 a 2$ & $a 2 b 2$ hsl7::hyg ${ }^{R}$ & This Study \\
\hline$h s l 7^{\text {Otef }}$ a2 & $a 2 b 2 P_{\text {olef }} h s l 7, c b x^{R}$ & This Study \\
\hline$\Delta s m u l$ a2 & $a 2 b 2 \operatorname{smul}:: c b x^{R}$ & This Study \\
\hline smu $1^{\text {Olef }}$ a2 & $a 2 b 2 P_{\text {otef }}-s m u 1, c b x^{R}$ & This Study \\
\hline$\Delta_{810-2832} h s l 7 \Delta s m u 1$ a2 & $a 2 b 2$ hsl7::hyg ${ }^{R}$, smul $:: c b x^{R}$ & This Study \\
\hline$\Delta_{810-2832} h s l 7 s m u 1^{\text {Otef }} a 2$ & $a 2 b 2$ hsl7::hyg ${ }^{R}, P_{\text {otef }}-s m u l, c b x^{R}$ & This Study \\
\hline \multicolumn{3}{|l|}{ Plasmids } \\
\hline pOtef-hsl7 & $P_{o t e f} h s l 7, c b x^{R}$ & This Study \\
\hline pOtef-smul & $P_{o t e f} S m u l, c b x^{R}$ & This Study \\
\hline pYES-hsl7 & $P_{G A L I}-h s l 7-U R A 3$ & This Study \\
\hline pGADT7-hsl7 & $P_{G A L, 4}-h s l 7-L E U$ & This Study \\
\hline pGBKT7-smul & 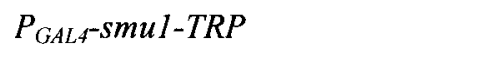 & This Study \\
\hline
\end{tabular}

\footnotetext{
${ }^{\mathrm{A}}$ All mutant strains were made in the wild type background strains listed.

can1-100, his3-11,15, leu2-3,112, lys2D::hisG, trp1-1, ura3-1, hsl7-420::HIS3) were a gift of Dr. J. Thorner (University of California, Berkeley), and MOSY 0150 (MATa, $\triangle$ ste20::TRP), and MOSY $0151(M A T \alpha, \Delta s t e 20:: T R P)$ were donated by Dr. D. Lew (Duke University). Escherichia coli strains, DH5 $\alpha$ (Bethesda Research Laboratories,
} 
Bethesda, MD) and TOP10 (Invitrogen, Carlsbad, CA) were utilized for all cloning and subcloning needs.

Ustilago maydis strains were grown at $25^{\circ} \mathrm{C}$ in YEP $(1 \%$ yeast extract, $2 \%$ peptone), supplemented with sucrose or dextrose, SLAD $(0.17 \%$ yeast nitrogen base without ammonium sulfate or amino acids (YNB), $2 \%$ dextrose, $50 \mu \mathrm{M}$ ammonium sulfate), and Minimal Media ( $0.17 \% \mathrm{YNB}, 2 \%$ dextrose, $0.3 \%$ ammonium nitrate, $6.25 \%$ Holliday Salt Solution (Holliday, 1974). All liquid cultures were grown with shaking (260 rpm). Mating media and solid media were made with $1 \%$ activated charcoal and/or 2\% agar (Holliday, 1974). Stressing media was made with calcofluor white (CFW, Fluorescent Brightener 28, Sigma, St. Louis, MO, $50 \mu \mathrm{M}$ ), congo red (CR, Fisher Scientific Company, Fairlawn, NJ, $15 \mu \mathrm{g} / \mathrm{mL}$ ), or sodium chloride (1 M).

Saccharomyces cerevisiae strains were grown at $30^{\circ} \mathrm{C}$ in YEPD or SD $(0.17 \%$ $\mathrm{YNB}, 1 \mathrm{X}$ amino acid drop out solution, the BD Matchmaker ${ }^{\mathrm{TM}}$ Library Construction \& Screening Kits user manual [BD Biosciences, Palo Alto, CA]) supplemented with dextrose or galactose. For complementation assays, yeast strains were grown in SD with either glucose (repressor of $\mathrm{P}_{G A L I}$ promoter) or galactose (inducer of $\mathrm{P}_{G A L I}$ promoter).

E. coli strains were grown at $37^{\circ} \mathrm{C}$ in $\mathrm{LB}$ (Luria-Bertani [Bertani, 1951]) and / or Circle Grow media (MP Biomedicals, LLC, Solon, OH).

Primer Design. Primers were designed with the Primer3 program (S. Rosen and H. J. Skaletsky, 1997 [http://frodo.wi.mit.edu/primer3/]). Primers were obtained from Eurofins MWG Operon (Huntsville, AL) and are listed in Table 2.

PCR. PCR and Gradient PCR was run on a PTC100 thermal controller (MJ Research Inc., San Francisco, CA) and a DNA Engine thermal cycler (Bio Rad 
TABLE 2. Primers used for PCR and Sequencing.

\begin{tabular}{ll} 
Primers & Sequences $\left(5^{\prime} \rightarrow 3^{\prime}\right)$ \\
\hline Hsl 5'2 & TGGCTTCACGCTCGTTCACT \\
Hsl 3'2 & AGGATCGGGGGCGATATTGT \\
cHsl Left & TTCACCATGCCGTCTTCC \\
cHsl Right & GATCGGGGGCGATATTGTA \\
Hsl Left & TCTGTCCGATCTCCAATTCC \\
Hsl Right & CCTCGTTGTCCTTCAATGGT \\
Hsl Sfi Up & TTCGGCCATCTAGGCCATGCCAAGGTAGGATGCGTAG \\
Hsl Sfi Down & TGAGGCCTGAGTGGCCCGAATATGGAACGGGAAATG \\
5Flank Left & GCATTCTCTTCGTCTGCTACCT \\
5Flank Right Sfi & TTCGGCCATCTAGGCCCAGCCTTTCGTGTTTGCTAC \\
NSmu5 & TTCTAGACTTCTCCTCCTCCTCCTCCT \\
NSmu3 & GATTGTTCGTGGTGCGAGTT \\
Smul000F & ACGCTCCATACCATCTCGTC \\
Smu1000R & GAACACTTTGAGGCAGCACA \\
\hline
\end{tabular}

Laboratories, Hercules, CA), respectively. PCR reactions were run with an initial denaturing temperature of $94^{\circ} \mathrm{C}$ for 4 minutes, followed by 34 cycles of a second denaturing temperature of $94^{\circ} \mathrm{C}$ for 30 seconds, annealing temperature ranging from $56^{\circ} \mathrm{C}$ to $62^{\circ} \mathrm{C}$ for 30 seconds, and extension temperature of $72^{\circ} \mathrm{C}$ for 1 minute per 1000 base pairs. A final extension temperature of $72^{\circ} \mathrm{C}$ for 2 minutes longer than the cycle extension was used to complete the reaction.

Comparison of $h s l 7 / s k b 1$ homologues. Amino acid sequences from five Hsl7 homologues found in Entrez Gene Database (http://www.ncbi.nlm.nih.gov/gene) were analyzed using the BLASTP tool from the $n c b i$ website (http://blast.ncbi.nlm.nih.gov/Blast.cgi). The sequences used were from Homo sapiens (PRMT5; Isoform A, accession no. NP_001034708.1 and Isoform B, accession no. NP_006100.2), Xenopus laevis (Hsl7; accession no. NP_001084480), 
Schizosaccharomyces pombe (Skb1; accession no. CAA17909), and Saccharomyces cerevisiae (Hsl7; accession no. NP_009691).

Genetic Manipulation and Vector Construction. PCR products were separated by gel electrophoresis on $0.6 \%$ agarose gel (Agarose LE, USB Corp, Cleveland, OH) and purified using the GeneClean III kit (MP Biomedicals, Solon, OH). PCR products were cloned into pCR2.1 TOPO vector (Invitrogen). Plasmid DNA was purified using the Wizard miniprep kit (Promega, Madison, WI). DNA samples were sequenced by the Nucleic Acids Core Facility (Center for Genetics and Molecular Medicine, University of Louisville, Louisville, KY), Eurofins MWG Operon (Huntsville, AL), or AGCT Inc. (Wheeling, IL). Restriction enzymes were purchased from New England Biolabs, Inc. (Bevelery, MA) or American Allied Biochemical, Inc. (Aurora, CO).

Deletion and overexpression of $h s l 7$ and smul in $U$. maydis were obtained by homologous recombination as described previously (Brachmann et al., 2004). The hsl7 deletion constructs were created using the SfiI technique (Brachmann et al., 2004; Kämper, 2004). Upstream (up-flank) and downstream (down-flank) fragments, approximately $1 \mathrm{~kb}$ in length each, of $h s l 7$ were amplified and used as flanking regions. Both carboxin and hygromycin resistance cassettes were used as markers and were cloned between the up and down flanks in the $S f i I$ sites. The $\Delta s m u l$ construct was created using a $4.4 \mathrm{~kb}$ PCR product from strain $10 / 18(\Delta s m u l$ alb1 in the 521 background, Smith et al., 2004) generated with primers smu1000F and smu1000R. The smul expression construct was produced by amplifying the $s m u l$ ORF with Nsmu5 and Nsmu3 primers and cloning the product into pCR2.1 TOPO (Invitrogen). To provide constitutive expression, the smul ORF was then excised and cloned after the $P_{\text {otef }}$ 
promoter into the BamHI and NotI sites of the p123 vector (Weber, et al., 2006). The $h s l 7$ expression vector was constructed by excising the $h s l 7$ ORF from pCR2.1-hsl7 and cloning it after the $P_{\text {otef }}$ promoter into the EcoRI site. Otef expression vectors were linearized using $S s p I$ before transforming U. maydis, with selection for carboxin resistance (Brachmann et al., 2001). Recombination at the ip locus would yield the carboxin resistant transformants. Disruption and over-expression constructs were either digested by restriction endonucleases, or a PCR product was amplified, purified, and used to transform $U$. maydis protoplasts. Potential transformants were verified by PCR.

Yeast two-hybrid analysis. Direct yeast two-hybrid assays were conducted following the BD Matchmaker ${ }^{\mathrm{TM}}$ Library Construction \& Screening Kits user manual (BD Biosciences). Two vectors, pGBKT7 (bait vector) and pGADT7 (prey vector), contain a series of restriction sites in frame with the 3' end of the GAL4 DNA-BD and the GAL4 DNA-AD, respectively, creating fusion proteins with proteins of interest. Directed assays were performed by introducing the bait and prey vectors into $S$. cerevisiae strain $\mathrm{AH} 109$ by co-transformation. A positive interaction was assessed after 3 days growth on -trp/-leu-/his-/ade synthetic drop out media (QDO). The prey fusion protein vector pGA-hsl7 was constructed by excising the $h s l 7 \mathrm{ORF}$ and cloning after the $\mathrm{P}_{\mathrm{GAL} 4}$ promoter into the $E c o R I$ site of pGADT7. The $s m u l$ ORF was excised and cloned after the $\mathrm{P}_{\mathrm{GAL} 4}$ promoter into the BamHI and NotI sites of pGBKT7.

Cell length, growth rate, cell viability on stressing media, mating assays, and plant pathogenesis. Cell density was measured spectrophotometrically in liquid culture. Exponential phase of growth $\left(\mathrm{OD}_{600}\right.$ of 0.5 to 0.7$)$ was obtained by measuring the optical densities of cells growing in liquid media for 24 hours. Then cultures were diluted to an 
optical density of 0.1 in fresh YEPS and allowed to grow for an additional $3.5 \mathrm{hrs}$. Cell length was measured in exponential growth $\left(\mathrm{OD}_{600}\right.$ of 0.5 to 0.7$)$ from cells grown in YEPS and analyzed using MetaMorph imaging software (MDS Analytical Technologies Inc., Mississauga, Ontario, Canada). Growth rate was determined by growing cell cultures in YEPS broth for 24 hours, then diluting to an $\mathrm{OD}_{600}$ of 0.023 in $50 \mathrm{~mL}$ of fresh YEPS. Cultures were allowed to stabilize for 4.5 hours, then optical densities were taken every hour for 11 hours. Stressor viability assays were performed by spotting $5 \mu \mathrm{L}$ of serial diluted cultures of exponentially growing cells, starting at a concentration of $10^{7}$ cells $/ \mathrm{mL}$ and decreasing to $10^{4}$ cells $/ \mathrm{mL}$. The cultures were spotted on media containing CFW $(50 \mu \mathrm{M}), \mathrm{CR}(15 \mu \mathrm{g} / \mathrm{mL})$, or sodium chloride $(1 \mathrm{M})$. Mating assays were performed using a concentration of $10^{7}$ cells $/ \mathrm{mL}$ and spotting $10 \mu \mathrm{L}$ on charcoal plates as previously described (Gold et al., 1997). Plant infection using 8 day old Golden Bantam corn seedlings (Bunton Seed Co., Louisville, KY and W. Atlee Burpee \& Co., Warminster, PA) was performed with a cell density of $1 \times 10^{5}$ cells / $\mathrm{mL}$ for haploid strains as previously described (Gold et al., 1997). Virulence was rated by a disease index on a scale of 0 to 5 with $0=$ no symptoms, $1=$ chlorosis and $/$ or anthocyanin biosynthesis, $2=$ small leaf galls, $3=$ small leaf and stem galls, $4=$ large galls, and $5=$ plant death. A disease rating was assigned to each plant 7, 10, 14, 17, and 21 days post inoculation ( +8 days post planting) and the indices for each strain were averaged to give a measure per strain. Three independent trials of approximately 20 plants were performed, averaged, and analyzed.

Statistical Analysis. Cell length measures were analyzed using one way ANOVA with a Dunnett's Multiple Comparison Test in Graphpad Prism 5.02 (Graphpad 
Software Inc., La Jolla, CA), while mating type specific comparisons were made using a Student's t-test in Microsoft Excel 2007 (Microsoft Corp., Redmond, WA). Growth rate data were analyzed using one way ANOVA with a Tukey's post hoc analysis in Graphpad Prism 5.02. Statistical analysis of the disease index measures were performed using a Kruskal-Wallis ANOVA with a Dunn's Multiple Comparison Test in Graphpad Prism 5.02.

Staining, micro-colonies, and microscopy. Staining of $U$. maydis cells in exponential growth $\left(\mathrm{OD}_{600}\right.$ of 0.5 to 0.7$)$ was obtained by treating $5 \mu \mathrm{L}$ of cells with $1 \mu \mathrm{L}$ of $10 \mu \mathrm{g} / \mathrm{mL}$ of CFW ( $2 \mu \mathrm{g} / \mathrm{mL}$; specific for $\beta$-glucan of cell wall) or $100 \mu \mathrm{g} / \mathrm{mL}$ wheat germ agglutinin (WGA, $17 \mu \mathrm{g} / \mathrm{mL}$; Tetramethylrhodamine conjugate, [Invitrogen], specific for chitin of cell wall,). Nucleic acid staining used $10 \mu \mathrm{M}$ Syto 11 (5 nM green fluorescent nucleic acid stain) or $1 \mu \mathrm{g} / \mathrm{mL}$ DAPI $(1 \mathrm{mg} / \mathrm{mL} 4,6-$ diamidino-2-phenylindole, blue cyan nucleic acid stain[Sigma]). Syto 11-stained cells were visualized using a FITC filter system. For visualization of CFW and DAPI stained cells, a UV-filter set was used. WGA stained cells were visualized using a TRITC filter set, and all stained images were taken on a Nikon Eclipse TE 200 Microscope (Nikon Inc., Melvin, NY) using MetaMorph imaging software. Non stained cell images were taken of cells in exponential growth $\left(\mathrm{OD}_{600}\right.$ of 0.5 to 0.7$)$ on a Nikon Eclipse $\mathrm{E} 800$ microscope using Spot imaging software (Diagnostic Instruments, Inc., Sterling Heights, MI) and analyzed using MetaMorph. To help visualize cell morphologies early in colony development, a micro-colony system was designed. Micro-colonies were prepared by inoculating $5 \mu \mathrm{L}$ of cell culture in exponential growth $\left(\mathrm{OD}_{600}\right.$ of 0.5 to 0.7$)$ onto a $60-80$ $\mu \mathrm{L}$ media spot on a sterile microscope slide and then covering with a sterile cover slip. 
The inoculated slides were placed, elevated, in a sterile glass petri dish with a small volume of sterile water (to prevent desiccation of media). After $48 \mathrm{hr}$ incubation (YEPS) or $72 \mathrm{hr}$ incubation (SLAD), slides were viewed under 10x, 20x, and / or 60x magnification on a Nikon Eclipse E800 microscope using Spot imaging software. Colony morphology images were taken on a Nikon Eclipse SMZ-U microscope using Spot imaging software. All photos were processed with Photoshop Elements 6 (Adobe, San Jose, CA).

\section{RESULTS}

\section{Smu1 plays a role in cell length and filamentation as a response to low}

ammonium conditions. Previous work (Smith et al, 2004) determined that Smul was involved in the mating response pathway, differentially influencing pheromone expression, and therefore is necessary for pathogenicity. It was also observed that Smul played a subtle role in the filamentous response (Smith et al., 2004). In addition, the phenotypes observed in the $\Delta s m u l$ mutant strains were mating-type specific, primarily affecting the a2b2 mating-type background. These mating-type specific phenotypes have also been observed for the U. maydis gene products Cla4 (Leveleki, et al., 2004) and Rhol (Pham, et al., 2009). However, the filamentous response to low ammonium conditions and cell morphology of smul mutant strains had not been examined in detail.

To explore these areas, smul disruption strains were generated by amplifying and purifying a $4.4 \mathrm{~kb}$ PCR fragment from strain 10/18 (4smul albl [background strain 521], Smith, et al., 2004) and transforming U. maydis cells to disrupt the target gene.

Disruptions were made in both FB1 $a l b 1$ and FB2 $a 2 b 2$ haploid background strains and confirmation of the positive gene integrations was made by PCR (data not shown). 
Overexpression of the smul ORF was obtained by amplifying and cloning the smul ORF after the $P_{\text {Otef }}$ promoter; the linearized plasmid was then integrated into the ip locus in $U$. maydis. The $s m u 1^{\text {Otef }}$ construct and its integration in the FB1 $a 1 b 1$ and FB2 a2b2 strains was also confirmed by PCR (data not shown).

$\Delta s m u 1$ strains generated in the FB background exhibited a reduction in the mating response primarily in the $a 2 b 2$ mating type background demonstrated previously in another genetic background (Fig. 5 and Smith et al., 2004). Building upon these results, I found that the $\Delta s m u l$ strains showed a decrease in cell length, though not statistically significant in $a 1 b 1$, while those with overexpression of the $s m u l$ ORF displayed elongated cell morphology in comparison to the wild type progenitor cells (Fig. 6A and Table 3). It was observed that the decrease in cell length showed statistical significance in the $a 2 b 2$ background only, while the $a l b 1$ strain was decreased cell length, but this was not statistically significant. The increase of cell length in the $s m u 1^{\text {Otef }}$ was only observed and statistically significant in the $a l b l$ background. Comparisons between the $a 1 b 1$ and $a 2 b 2 s m u 1$ mutant strains indicated that the differences between the mating specific strains were significant in both the disruption and overexpression strains (Table 4). These results are consistent with the mating-type specific phenotypes previously observed in the $s m u 1$ mutant backgrounds.

The Smu1 homolog from $S$. pombe, Shk1p, plays a role in cell cycle regulation displaying a decrease in cell length when shklexpression is repressed, while increasing in cell length when shkl is overexpressed (Gilbreth, et al., 1998). This is similar to what has been observed in smul mutants. To explore additional roles Smul might play in the cell cycle, the growth rates of $\Delta s m u l$ and $s m u 1^{\text {Otef }}$ strains were examined. $\Delta s m u l, s m u 1^{\text {Otef }}$, 

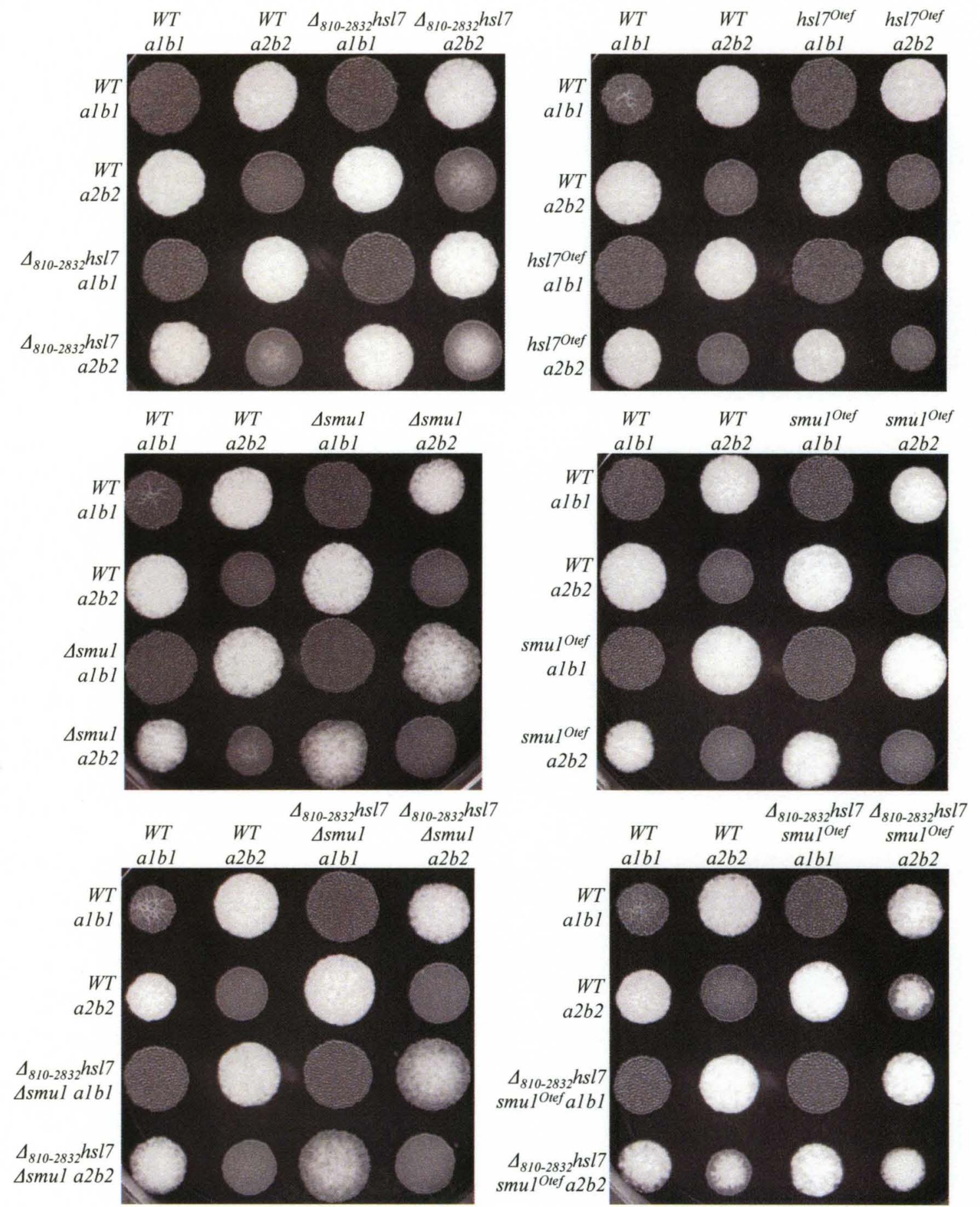

Figure 5: Plate mating assays for $\Delta_{810-2832} h s l 7, h s l 7^{\text {Otef }}, \Delta s m u l, s m u I^{\text {Otef }}, \Delta_{810-2832} h s l 7 \Delta_{s m u l,} \Delta_{810}$ ${ }_{2832} \mathrm{hsl}_{\mathrm{S}} \mathrm{smul}{ }^{\text {Otef }}$ strains. Equal mixtures of haploid strains of opposite mating-type background were plated onto YPD plates containing activated charcoal. A positive mating reaction produced a white "fuz" phenotype of aerial hyphae production. See text for description of results. 
TABLE 3. Measures of cell length across all strains.

\begin{tabular}{|c|c|c|c|c|}
\hline Strain & $\mathrm{n}$ & Length $^{\mathrm{A}}$ (in $\left.\mu \mathrm{m}\right)$ & Comparison & $p^{B}$ \\
\hline WT albl & 91 & $19.27+/-.43$ & WT alb1 v. WT a2b2 & N.S. \\
\hline$\Delta_{810-2832} h s l 7$ al & 167 & $25.22+/-.45$ & WT albl v. $\Delta_{810-2832} h s l 7$ al & $>.001$ \\
\hline$h s l 7^{\text {Otef }}$ al & 227 & $18.66+/-.27$ & WT albl v. hsl $7_{\text {Otef }}^{\text {al }}$ & N.S. \\
\hline$\Delta s m u l$ al & 380 & $18.64+/-.16$ & WT albl v. $\Delta s m u l$ al & N.S. \\
\hline$s m u I^{\text {Otef }}$ al & 308 & $21.75+/-.28$ & WT albl v. smul ${ }^{\text {Otef }}$ al & $>.001$ \\
\hline$\Delta_{810-2832} h s l 7 \Delta s m u l$ al & 221 & $25.25+/-.33$ & WT alb1 v. $\Delta_{810-2832} h s l 7$ Asmul al & $>.001$ \\
\hline$\Delta_{810-2832} h s l 7 s m u l^{O t e f} a l$ & 143 & $27.86+/-.45$ & WT albl v. $\Delta_{810-2832} h s l 7$ smul Otef $a l$ & $>.001$ \\
\hline$W T a 2 b 2$ & 213 & $19.14+/-.31$ & & \\
\hline$\Delta_{810-2832} h s l 7$ a2 & 406 & $24.78+/-.32$ & $W T a 2 b 2$ v. $\Delta_{810-2832} h s l 7$ a2 & $>.001$ \\
\hline$h s l 7^{\text {Otef }} a 2$ & 173 & $17.31+/-.29$ & WT $a 2 b 2$ v. $h s l 7^{\text {Otef }} a 2$ & $>.01$ \\
\hline Asmul a2 & 281 & $17.64+/-.18$ & $W T a 2 b 2$ v. $\Delta s m u 1$ a2 & $>.05$ \\
\hline smul ${ }^{\text {Otef }} a 2$ & 224 & $19.66+/-.30$ & $W T a 2 b 2$ v. smu $1^{\text {Otef }} a 2$ & N.S. \\
\hline$\Delta_{810-2832} h s l 7 \Delta s m u l a 2$ & 137 & $24.25+/-.45$ & $W T a 2 b 2$ v. $\Delta_{810-2832} h s l 7 \Delta s m u 1$ a2 & $>.001$ \\
\hline$\Delta_{810-2832} h s l 7 s^{s m u l^{O l e f}}$ a2 & 89 & $27.33+/-.86$ & WTa2b2 v. $\Delta_{810-2832} h s l 7 s m u I^{\text {Otef }}$ a2 & $>.001$ \\
\hline
\end{tabular}

${ }^{\mathrm{A}}$ Cell length values are averages, +/- S.E.

B Statistical analysis was performed using a one way ANOVA with a Dunnett's Multiple Comparison Test.

N.S. $=$ Not Significant

TABLE 4. Mating-type specific cell length differences.

\begin{tabular}{|c|c|c|c|c|}
\hline Strain & $\mathrm{n}$ & Length $^{A}$ (in $\mu \mathrm{m}$ ) & Comparison & $p^{B}$ \\
\hline$\Delta s m u l$ al & 380 & $18.64+/-.16$ & & \\
\hline$\Delta s m u l a 2$ & 281 & $17.64+/-.18$ & $\Delta s m u l$ al v. $\Delta s m u l$ a2 & $>.001$ \\
\hline smul ${ }^{\text {Otef }}$ al & 308 & $21.75+/-.28$ & & \\
\hline$s m u I^{\text {Olef }}$ a2 & 224 & $19.66+/-.30$ & smul $l^{\text {Otef }}$ al v. smul $l^{\text {olef }}$ a 2 & $>.001$ \\
\hline$h s l 7^{\text {otef }}$ al & 227 & $18.66+/-.27$ & & \\
\hline$h s l 7^{\text {Otef }} a 2$ & 173 & $17.31+/-.29$ & $h s l 7^{\text {Otef }}$ al v. $h s l 7^{\text {Otef }}$ a2 & $>.001$ \\
\hline
\end{tabular}

${ }^{A}$ Cell length values are averages, +/- S.E.

B Statistical analysis was performed using a Student's t-test.

N.S. $=$ Not Significant 

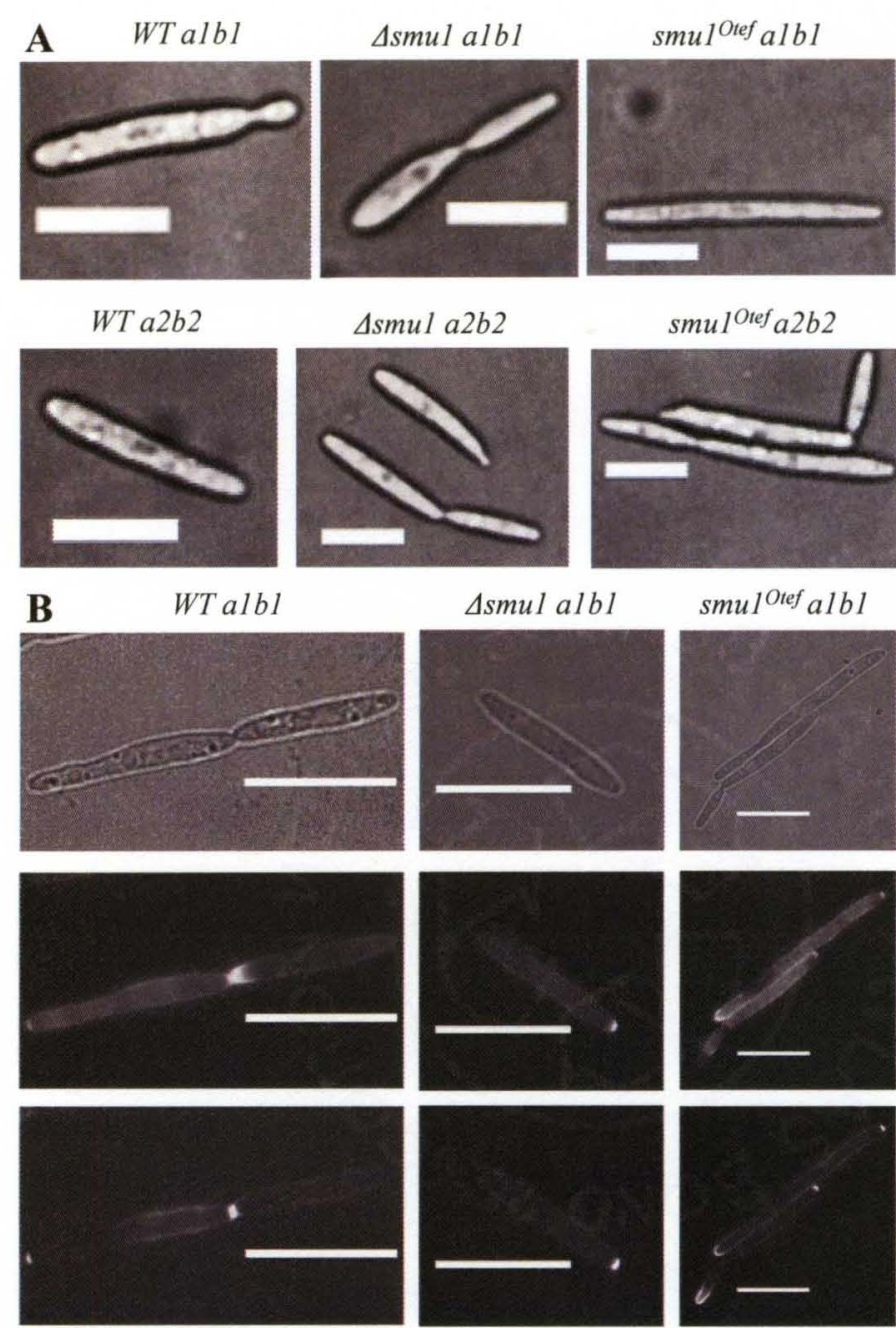

WT $a 2 b 2$
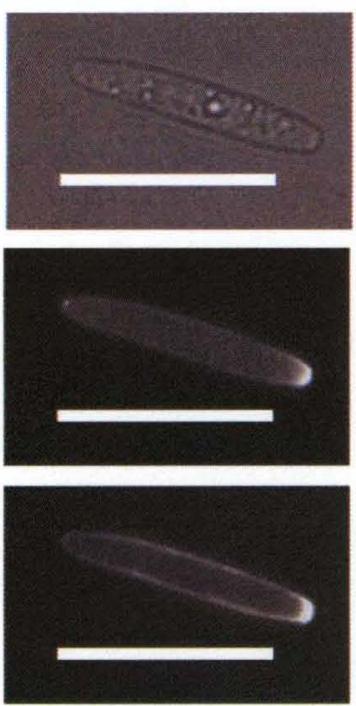

$\Delta s m u 1$ a2b2
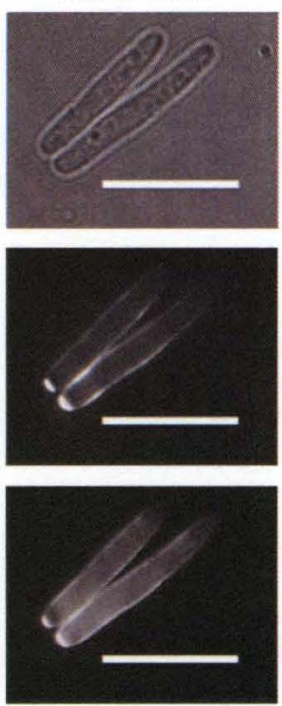

smul $^{\text {Otef }} a 2 b 2$

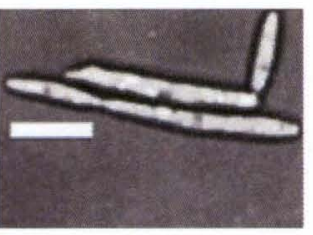

$$
\text { smul Otef } a l b l
$$
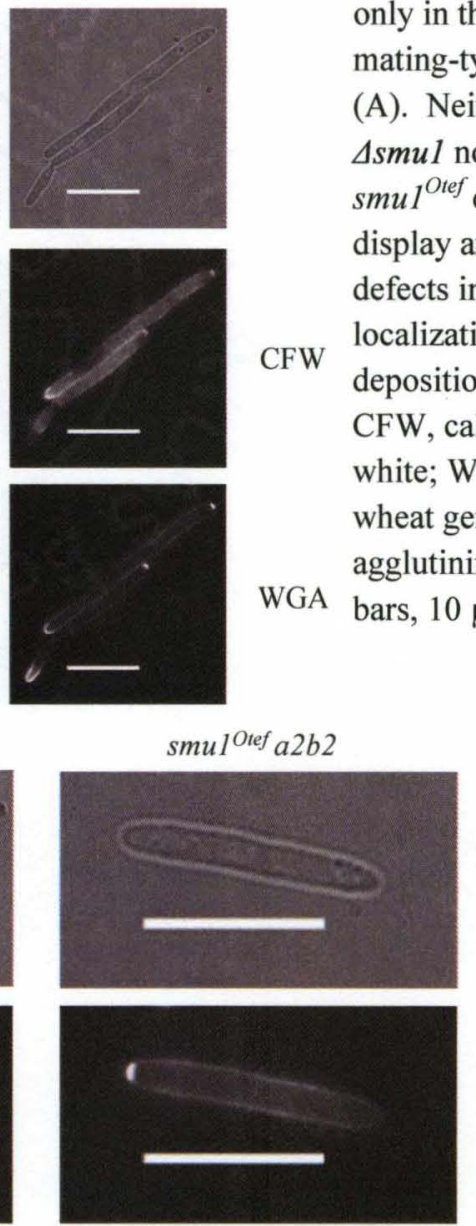

CFW

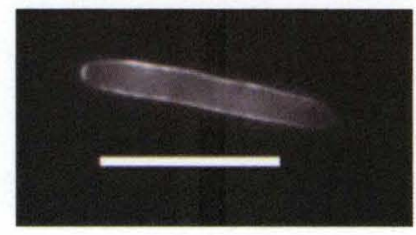
WGA bars, $10 \mu \mathrm{m}$.
Figure 6: Smu1 affects cell length but not cell wall localization. $\Delta s m u 1$ cells display a decrease in cell length primarily in the $a 2 b 2$ mating-type strain while smu1 ${ }^{\text {tref }}$ cells exhibit an increase in cell length but only in the $a l b 1$ mating-type strain (A). Neither $\triangle s m u 1$ nor smu $1^{\text {Otef }}$ cells display any defects in cell wall localization and deposition (B). CFW, calcofluor white; WGA, wheat germ agglutinin. Scale 
and wild type cells were grown in YEPS media and optical densities were taken every hour for eleven hours. The optical densities were plotted in Excel and doubling times were determined and analyzed (described in Materials and methods). Examination of rates of growth of the smul mutants indicated no differences from those of the wild type progenitor strains (Table 5).

TABLE 5. Growth rate analysis of mutant strains. ${ }^{A}$

\begin{tabular}{|c|c|c|}
\hline Strain & Double Time $^{\mathrm{B}}$ & $p^{C}$ \\
\hline WT albl & $2.76+/-.18$ & \\
\hline$\Delta s m u l$ al & $2.79+/-.16$ & 0.82 \\
\hline smul Itef al & $2.81+/-.10$ & 0.56 \\
\hline$\Delta_{810-2832} h s l 7$ al & $2.68+/-.15$ & 0.43 \\
\hline$h s l 7^{\text {otef }}$ al & $2.71+/-.13$ & 0.56 \\
\hline$\Delta_{810-2832} h s l 7 \Delta s m u l$ al & $2.86+/-.19$ & 0.38 \\
\hline$\Delta_{810-2832} h s l 7 s m u l^{\text {Otef }}$ al & $2.65+/-.12$ & 0.22 \\
\hline$W T a 2 b 2$ & $2.77+/-.20$ & 0.95 \\
\hline$\Delta s m u l$ a2 & $2.74+/-.12$ & 0.75 \\
\hline smu $1^{\text {Otef }}$ a2 & $2.61+/-.15$ & 0.14 \\
\hline$\Delta_{810-2832} h s l 7$ a2 & $2.74+/-.16$ & 0.79 \\
\hline$h s l 7^{\text {Otef }} a 2$ & $2.81+/-.13$ & 0.69 \\
\hline$\Delta_{810-2832} h s l 7$ Аsmul a2 & $2.83+/-.15$ & 0.55 \\
\hline$\Delta_{810-2832} h s l 7 s m u 1^{\text {Oref }} a 2$ & $2.94+/-.14$ & 0.11 \\
\hline
\end{tabular}

A Table shows growth rate analysis of six identical experiments per strain.

${ }^{B}$ Growth rate expressed as doubling time in hours, +/- S.E.

C Statistical analysis was performed using a one way ANOVA with a Tukey's Multiple Comparison Test.

Another possible cause of the cell elongation could be misappropriation of cell wall components. Staining of both $\Delta s m u l$ and $s m u 1^{\text {Otef }}$ strains with calcofluor white (CFW, a beta-glucan specific stain and cell wall stressor) and wheat germ agglutinin (WGA, chitin specific stain) did not show any cell wall aberrations, indicating that cell 
wall material was able to localize and deposit correctly to the growing tip and in the mother daughter septa (Fig. 6B). Thus, Smul does not appear to play a role in cell cycle regulation or cell wall localization and deposition, however work still needs to be done to explore these effects on cell length.

Previous work indicated that Smu1 plays a role in the filamentous response to low ammonium conditions (Smith et al., 2004). Colony morphology of smul mutant strains compared to their wild type progenitors was examined, via plate colonies and micro-colonies. Strains were grown on either YEPS or low ammonium (SLAD; $50 \mu \mathrm{M}$ ammonium) media. Colony morphology of $\Delta s m u 1$ and $s m u 1^{\text {Otef }}$ mutant strains on YEPS displayed no differences when compared to their wild type progenitor strains (Fig. 7A). To further explore the differences in colony morphology, examination of micro-colonies was utilized and enabled a closer view of the responses of the strains on the two types of media. Micro-colonies were generated by inoculating a media spot on a sterile microscope slide, covered with a sterile cover slip, incubating for $2-3$ days at $25^{\circ} \mathrm{C}$, and then examining under magnification. On YEPS, both at 20x and 60x magnification, neither $\Delta s m u 1$ nor $s m u 1^{\text {Otef }}$ mutant strains displayed any phenotypic difference from that of the wild type progenitor strains (Fig. 7B). $\Delta s m u 1$ mutant strains displayed a subtle decrease in the filamentous response when grown on SLAD and this decrease appears to be more substantial in the $a 2 b 2$ background. Overexpression strains, on the other hand, showed an increase in the production of filaments on SLAD and this appeared to be greater in the albl background (Fig. 8A and Smith et al., 2004). When grown on SLAD micro-colonies and viewed at 20x magnification, $\Delta s m u l$ strains displayed a decrease in filament formation, which is more severe in the $a 2 b 2$ background (Fig. 8B, Inset). 


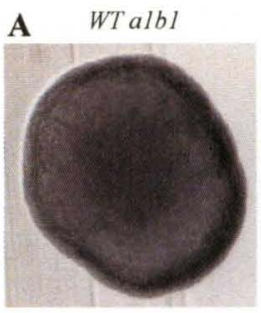

WT $a 2 b 2$

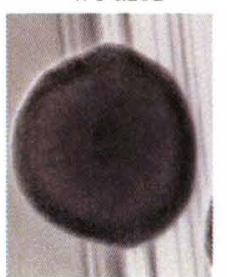

B

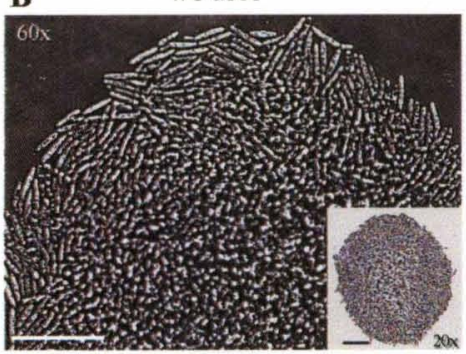

WT $a 2 b 2$

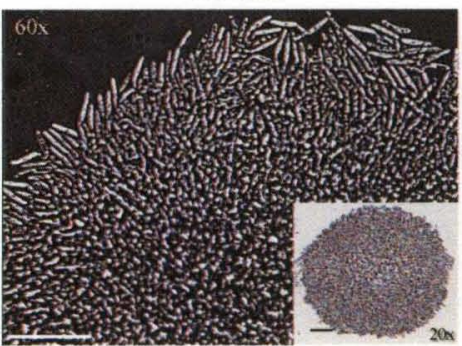

$\triangle \operatorname{smul} a 2 b 2$ smul Olef $a l b 1$
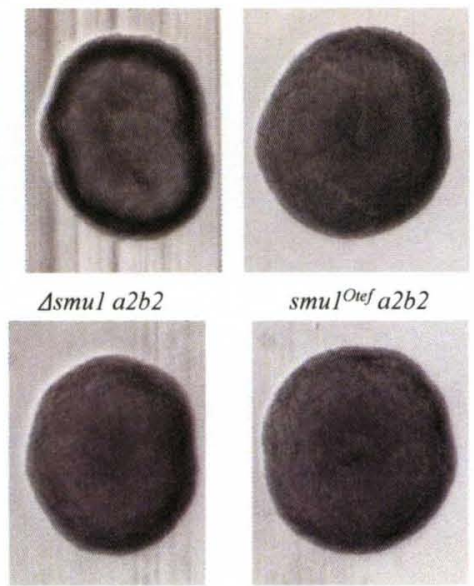

$\triangle s m u l a l b l$

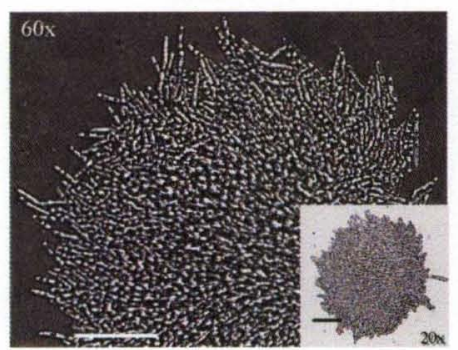

$\triangle s m u l a l b 1$

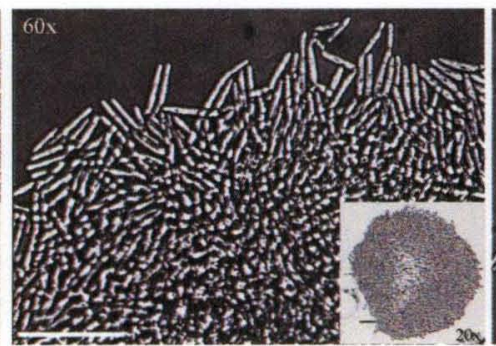

Figure 7: smu1 mutant strains did not display any defects in colony morphology on rich media. Grown on rich media, the morphology of both the $\Delta s m u l$ and $s m u 1^{\text {Otef }}$ mutant strain colonies did not differ from that of the wild type strains, producing smooth colonies (A). Examination of micro-colonies of the $\Delta s m u l$ and $s m u 1^{\text {Olef }}$ mutant strains did not indicate any difference compared to the wild type strains (B). Scale bars, $50 \mu \mathrm{m}-$ $20 \mathrm{x}$ inset image, $40 \mu \mathrm{m}-60 \mathrm{x}$ image.

At 60x magnification, the mating-type specific decrease is more evident. smul ${ }^{\text {Otef }}$ mutant strain filaments were longer than those observed in the wild type progenitor cells and the effect is again mating-type specific with the $a l b l$ mutant background strain producing filaments above what is seen in the $a 2 b 2$ mutant background strain (Fig. 8B).

Examination of sensitivity of the smul mutants to the cell wall antagonists CFW and CR indicated no difference to the wild type progenitor strains (data not shown). Acid $\mathrm{pH}$ also had no affect on smu1 mutants (Smith, et al., 2004). Overall, these results indicate that 


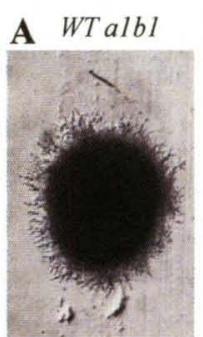

WT $a 2 b 2$

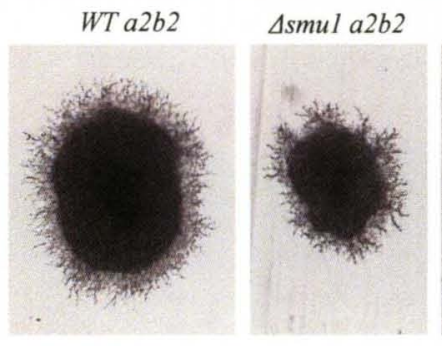

B

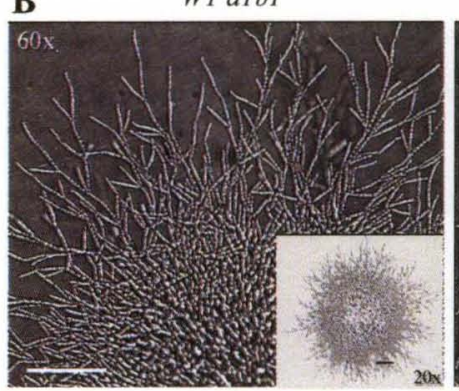

WT $a 2 b 2$

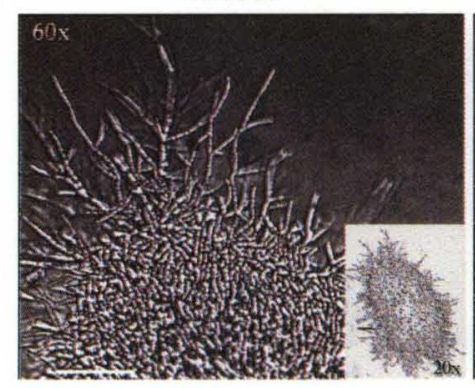

smul ${ }^{\text {Otef }}$ albl

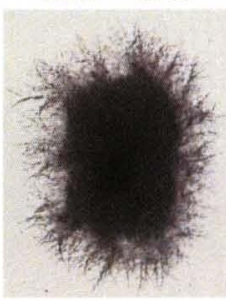

smul $1^{\text {Otef }} a 2 b 2$

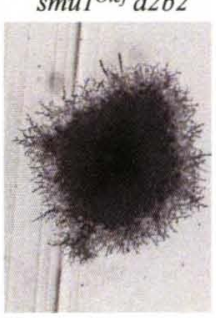

Figure 8: Filamentous response to low

ammonium conditions of smul mutants. Strains were grown on SLAD media and examined for filament formation. $\Delta s m u l$ colonies display a decrease in the ability to form filaments primarily in the $a 2 b 2$ mating-type background while $s m u 1^{\text {Otef }}$ colonies exhibit an increase in filament formation but only in the albl mating-type background (A). Micro-colonies were utilized to examine filament formation at the cellular level. $\Delta s m u l$ strains exhibited decreased filament length and number of filaments with a more severe reduction in the $a 2 b 2$ mating-type background. The albl smul ${ }^{\text {Otef }}$ strain displayed an increase in both filament length and number of filaments (B). Scale bars, $50 \mu \mathrm{m}-20 \mathrm{x}$
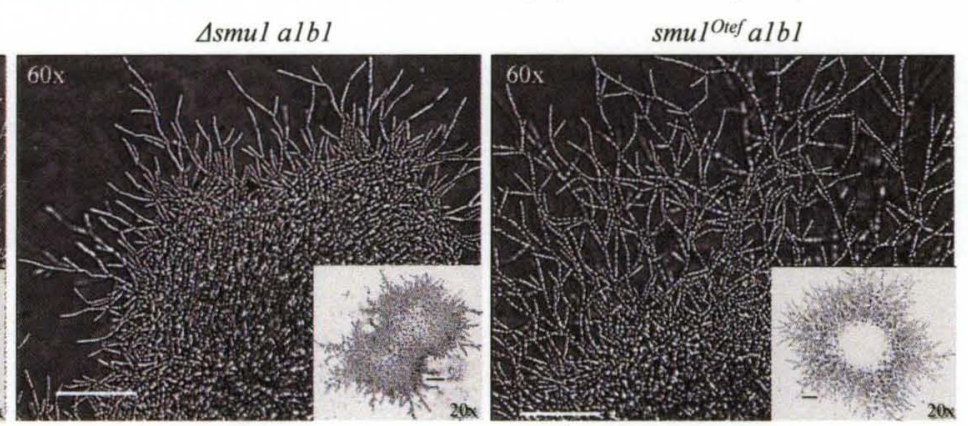

$\triangle s m u l a 2 b 2$

smul ${ }^{\text {Ote }}$ a $2 b 2$
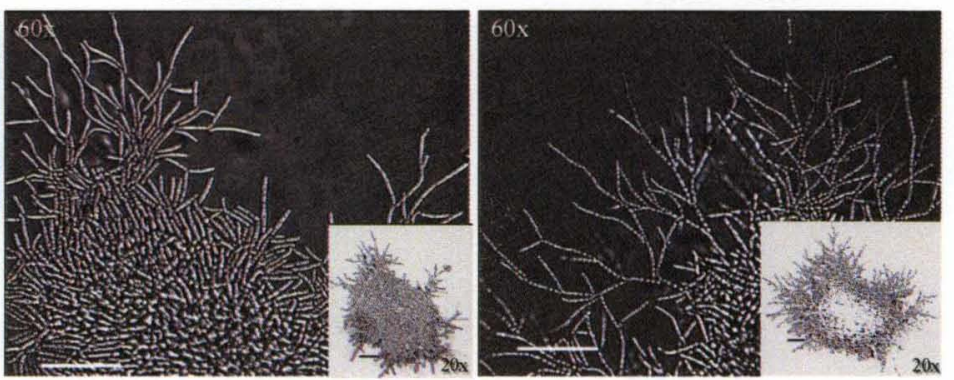

Smu1 plays a role in cell length and the filamentous response pathway to low ammonium conditions, in addition to the mating response in $U$. maydis.

\section{Identification of an Ustilago maydis Hsl7 homolog and construction of hsl7}

specific mutants. As described in the introduction, a putative protein-arginine

$\mathrm{N}$-methyltransferase homolog (um15057) was identified in U. maydis through a search of

the newly available $U$. maydis genome databases (first through an agreement with

Exelixis, Inc.; now available publicly through the Whitehead Institute's genome 
initiatives and the MumDB Website [http://mips.helmholtz-

muenchen.de/genre/proj/Ustilago]). Primers were designed to amplify a $3 \mathrm{~kb}$ region of

DNA from genomic DNA. A BLASTX comparison of the predicted protein showed

similarity to various $h s l 7 / s k b l$ homologues. Annotation on the MumDB website

identified a predicted ORF from which primers were designed to amplify a $2.9 \mathrm{~kb}$ ORF

from cDNA. BLASTP comparison to other $h s l 7 / s k b l$ homologues showed greatest

similarity to the human protein PRMT5 [Isoform A, accession no. NP_001034708.1 and

Isoform B, accession no. NP_006100.2; Identities $=207 / 473(43 \%)$, Positives $=277 / 473$

$(58 \%)$, Gaps $=41 / 473(8 \%)]$, Schizosaccharomyces pombe protein Skb1 [accession no.

CAA17909; Identities $=215 / 507(42 \%)$, Positives $=290 / 507(57 \%)$, Gaps $=33 / 507$

(6\%)], and Xenopus laevis protein Hsl7 [accession no. NP_001084480; Identities =

201/471 (42\%), Positives $=273 / 471(57 \%)$, Gaps $=41 / 471(8 \%)]$. Similarity was also

seen to the Saccharomyces cerevisiae protein Hs17 [accession no. NP_009691; Identities

$=205 / 660(31 \%)$, Positives $=303 / 660(45 \%)$, Gaps $=130 / 660(19 \%)]$. A search of

\begin{tabular}{|c|c|c|}
\hline Prmt5A|H.sap & PLOPLMDNLESQTYEVFEKDP I KYSOYOQAIYKCILDRVPEEERDTNVOVIMVLGAGRGD & 370 \\
\hline Prmt 5B $\{$ H. sap & PLORLMDNLE SQTYEVFEKDP I KYSQYOOAIYKCLLDRVPEEERDTNVOVIMVLGAGRGP & \\
\hline HsI7|X.Iae & PLQPLMDNLESOTYEVFEKDPVKYSOYOOAVYKCLLDRVPEEEKETNIOIIMVLGAGRGP & 6 \\
\hline Hs $17 \mid$ U . may & PLOPLMDNLEGTTYEVFERDPVKYALYEEAVYKALLDRPVTAN-----TL TVCGAGRGP & 387 \\
\hline skblis pom & PLOPLSYNLENI TYEI FERDPVKYAOYEOA I FSAIMDRD-----ESSVTR |AVVGAGRGP & 357 \\
\hline \multirow[t]{2}{*}{ Hsl7|s.cer } & PLKPHSDNLLNSTYLTFEKDLVKYDLYESAILEALODLAPRAS-AKRPLV LVAGAGRGP & \\
\hline & 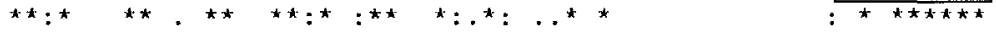 & \\
\hline Prmt 5A|h.sap & LVNASLRAAKQ--ADRRI KLYAVEKN PNAVVTLENWOEEEWGSOVTVVSSDMREWVAP-- & 42 \\
\hline Prmt 5B|H.sap & IVNASLRAARQ--ADRRIKLYAVEKNPNAVVTLENWOFEEWGSQVTVVSSDMREWVAP-- & 409 \\
\hline Hsil IX.lae & LVNASLRAAKQ - -AERKIKVYAVEKNPNAV ITLEGWRYEEWGSQVTVVSGDMREWKAP-- & 42 \\
\hline Hs.l7/U.may & LVDRCLNAADR--AGRAVRLVALERNDNALVTLQERQALEWGDQVKVOYGDMRKYPVPSS & \\
\hline skb1 / s.pam & LVDCALRAA IS--SSRTVDMIALEKN PNAFSMLLMRNRODWAGKVTEVFGDMRTWNDD-- & 41.3 \\
\hline \multirow[t]{2}{*}{ Hsl7is.cer } & LVDRTFKI ISMLEMDSKVS I I IEKNPQAYLYLOKRNFDCWDNRVKLIKEDMTKWOINEP & 45 \\
\hline & 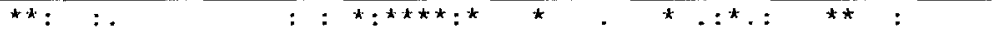 & \\
\hline Prmt 5 A $\mid$ H. sap & ---EKADIIVSELLGSFADNELSPECLDGAQHELKDD-GVSI PGEYTSFLAPISSSKLY & 48 \\
\hline Prmt5B|H. sap & ---EKADIIVSELLGSFADNELSPECLDGAOHFLKDD-GVSIPGEYTSFLAPISSSKLY & 46 \\
\hline Hsl7|X.lae & - --EKADIIVSELLGSFGDNELSPECLDGAOHFIKDD-GVSIPGEYTSYLAPISSSKLY & 477 \\
\hline Hs $17 \mid \mathrm{U} . \mathrm{may}$ & MA--ERPDIVVSELIGSFGDNELSPECLDGAMRFLKPN-GISIISSSYTAFLSPLSSEKLA & 502 \\
\hline skb1 is.pom & - --YKIDILVSELIGSMGDNELSPECLDGVOHVIDEETGICIPSSYISYVTPIMSPKLW & 46 \\
\hline Hsl7|S.cer & SEKRIOIDLCISELLGSFGCNELSPECLWSIEKYHSHNDTIEI PRSYSSYIAPI SSPLFY & 51 \\
\hline
\end{tabular}

Figure 9: Hsl7 is a conserved protein arginine methyltransferase. Protein sequence alignment of the single methyltransferase domain (highlighted) indicates that Hs17 aligns with other known members of the methyltransferase family. 
potential domains in the sequence from $U$. maydis yielded only one hit, the Methyltransf_12 domain (Fig. 9). This region showed the highest similarity amongst the homologues with PRMT5 isoforms A and B and Hs17 from Xenopus, (Identities $=64 / 105$ $(60 \%)$, Positives $=77 / 105(73 \%)$, Gaps $=4 / 105(3 \%)$ and Identities $=63 / 105(60 \%)$, Positives $=79 / 105(75 \%)$, Gaps $=4 / 105(3 \%)$, respectively $).$ Skb1 was next in similarity, (Identities $=53 / 99(53 \%)$, Positives $=64 / 99(64 \%)$, Gaps $=4 / 99(4 \%)$, followed by Hs17 from $S$. cerevisiae, Identities $=47 / 106(44 \%)$, Positives $=62 / 106(58 \%)$, Gaps $=4 / 106$ $(3 \%)$.

Hs17 interacts with Smu1 in vitro. Hs17 homologues have been shown to interact with Ste20-like p21-activated kinases (PAKs) in both S. cerevisiae and S. pombe and to play a role in the filamentous response to low ammonium conditions as well as cell cycle regulation and response to hyperosmotic conditions (Ma, et al., 1996; Gilbreth, et al., 1996 and 1998; Fujita, et al., 1999; Bao, et al., 2001). To determine if Hsl7 from $U$. maydis interacts with Smul, the Ste20 PAK homolog in U. maydis, yeast two-hybrid analysis was utilized. The ORF's of both $h s l 7$ and $s m u l$ were cloned after the Gal4 promoter of yeast based vectors. The $h s l 7$ ORF was cloned into pGADT7 "prey" vector and fused to the activation domain of the Gal4 transcription factor, while the smul ORF was cloned into the pGBKT7 "bait" vector and fused to the DNA binding domain of the Gal4 transcription factor. During a positive reaction the two proteins of interest interact, reconstituting the transcription factor and activating reporter genes, allowing for growth on auxotrophic media. Both pGA-hsl7 and pGB-smul vectors were co-transformed into the AH109 yeast background strain and plated onto double dropout media (DDO), with growth confirming successful transformation of both plasmids. Several colonies were 
subsequently restreaked on to quadruple dropout media (QDO), a more stringent selective media and a positive interaction was determined by colony growth. Through this process it was observed that Hsl7 and Smu1 interacted in vitro, growing on QDO. Two other proteins known not to interact with Hsl7, Rho1 and Pdc1 (Pham, et al., 2009), served as negative controls; when these were used in yeast two-hybrid experiments against Hsl7, the transformants failed to grow on QDO. Smu1 and Rho1, which were previously shown to interact in vitro (Pham, et al., 2009), did show a positive interaction, growing on QDO (Fig. 10). This is a strong indication that Hsl7 and Smul potentially interact in vivo.

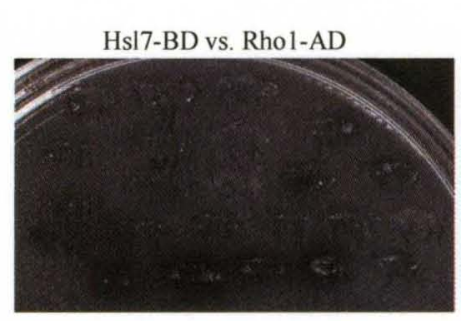

Hsl7-BD vs. Pdel-AD

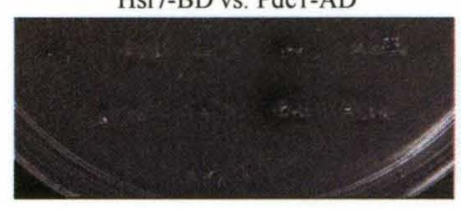

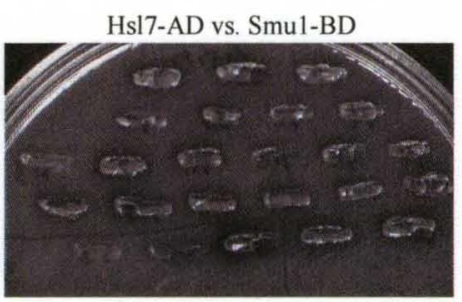

Rho1-AD vs. Smul-BD

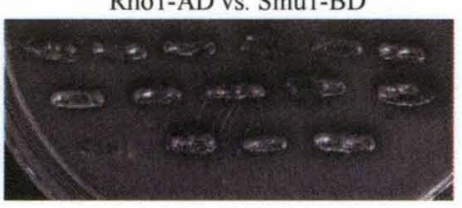

Figure 10: Hsl7 interacts with Smu1 in vitro. Yeast two-hybrid analysis of Hsl7 and Smul interaction. Hsl7, cloned in pGADT7, and Smu1, cloned into pGBKT7, were co-transformed into AH109; a positive interaction is indicated by growth on restrictive media. Hs17, cloned into pGBKT7 was tested against both Rhol and Pdcl, cloned into pGADT7 as negative controls. pGB-Smul was tested against pGA-Rhol as a positive control.

\section{Disruption of $h s / 7$ increases cell length and the filamentous response in low}

ammonium conditions. From the sequence identified as $h s l 7$, two disruption constructs were created. These two disruption constructs were produced using an $S f i$-based approach by inserting either a carboxin or hygromycin resistance cassette between an upstream and downstream flank of genomic DNA. The constructs were excised by restriction endonucleases, purified and used to transform U. maydis. Disruptions of hsl7 were made in both an FB1 alb1 haploid strain and an FB2 a2b2 haploid strain and confirmation of the gene disruptions were confirmed by PCR (data not shown). The 
initial construct was created before the MumDB website annotated the $h s l 7 \mathrm{ORF}$. This construct retains the first 810 base pairs of the ORF. After annotation of the $h s l 7$ ORF was completed on the MumDB website and confirmed in our lab by sequencing of cDNA (data not shown), a complete deletion of the hsl7 ORF was generated. All further examination did not reveal any phenotypic differences between strains with complete deletion and partial disruption constructs. Thus, the remainder of the experiments will focus on the partial disruption strains $\left(\Delta_{810-2832} h s l 7\right)$. Overexpression of the $h s l 7 \mathrm{ORF}$ was obtained by first amplifying and cloning the $h s l 7 \mathrm{ORF}$ after the $P_{\text {Otef }}$ promoter; the linearized plasmid was integrated via recombination into the ip locus in both the $a 1 b 1$ and $a 2 b 2 \mathrm{FB}$ strains of $U$. maydis. Generation of the appropriate construct and transformants was confirmed by PCR (data not shown). The fact that deleting $h s l 7$ in haploid strains still allowed cell growth and reproduction indicates that Hsl7 is not essential for cell viability.

Disruption strains $\left(\Delta_{810-2832} h s l 7\right)$ displayed elongated cell morphology, while overexpression of the $h s l 7 \mathrm{ORF}$ showed a decrease in cell length compared to the wild types (Fig. 11A and Table 3). Statistical evidence indicates that the elongated cell length of the disruption strains is significantly different from the wild type progenitor strains, independent of mating-type background. In addition, there was no significant difference between the $\Delta_{810-2832} h s l 7 a 1 b 1$ and $\Delta_{810-2832} h s l 7$ a2b2 strains. The cell length decrease was significant in the $a 2 b 2 h s l 7^{\text {ote }}$ strain only. The comparison between the $a l b l$ and $a 2 b 2 \mathrm{hsl}^{\text {Otef }}$ strains indicated that the difference in cell length between the mating-type specific mutant strains was significant (Table 4). This is an interesting result, potentially 

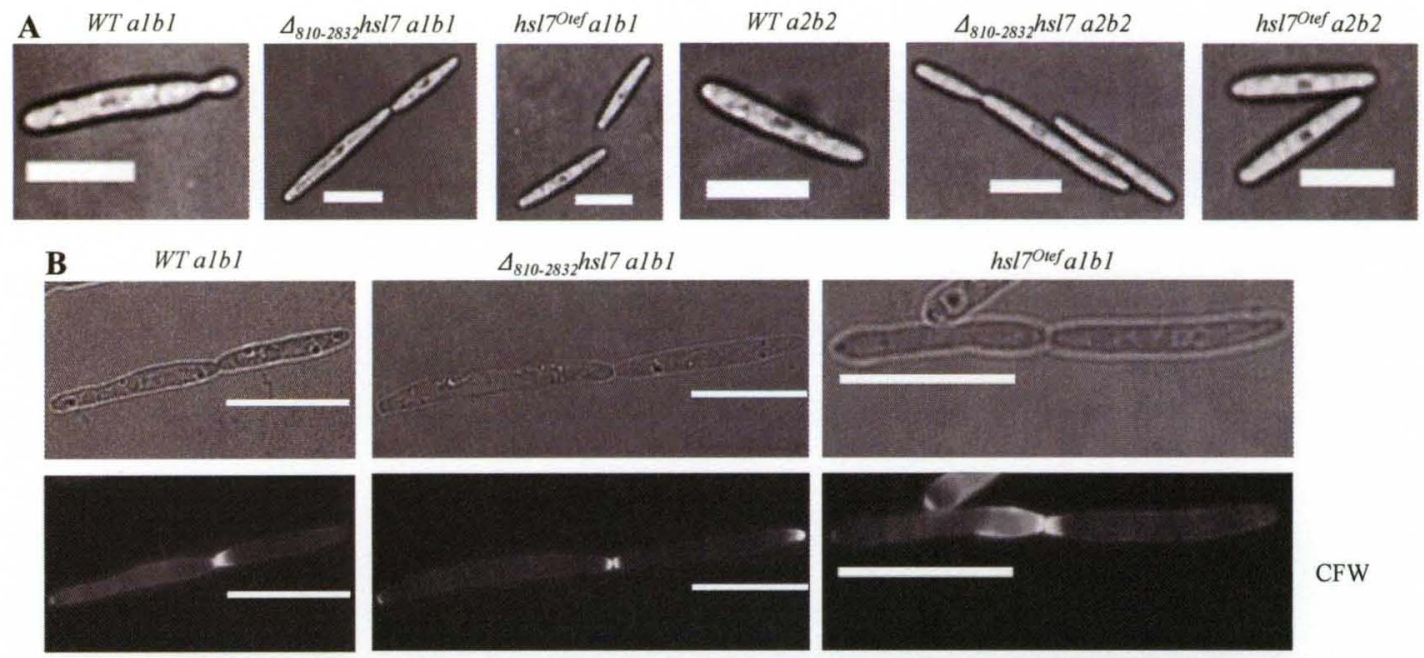

CFW
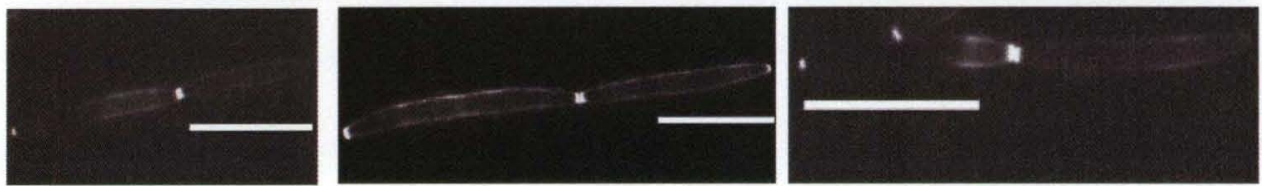

WGA
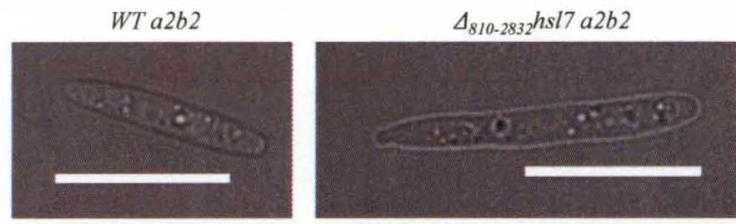

$h s l 7^{\text {Otef }} a 2 b 2$
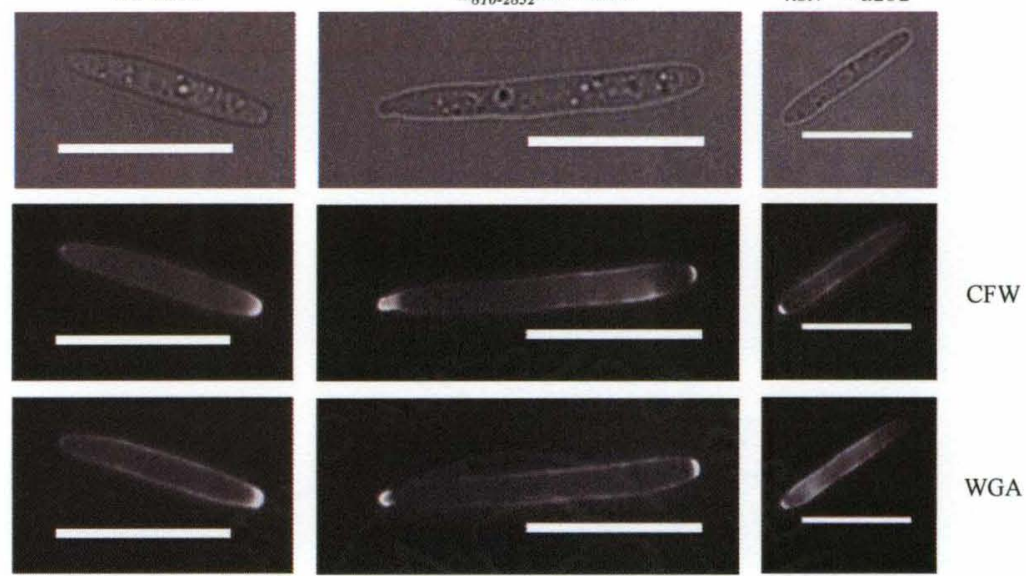

Figure 11: Hsl7 affects cell length but not cell wall localization. 4 hsl 7 cells display an increase in cell length while $h s l 7^{\text {Otef }}$ cells exhibit a decrease in cell length but only in the alb1 mating-type strain (A). Neither $\Delta h s l 7$ nor $h s l 7^{\text {Otef }}$ cells display any defects in cell wall localization (B). Scale bars, $10 \mu \mathrm{m}$.

adding Hs17 to a growing list of proteins (e.g. Smu1, Cla4, and Rho1) whose expression differently affects haploids of one mating-type over the other.

The effect of Hsl7 on cell length is opposite to a phenotype observed in $s k b 1$ mutant strains of $S$. pombe. $\Delta s k b 1$ strains were decreased in cell length, where as $s k b 1$ overexpression strains were increased in cell length (Gilbreth, et al., 1996). The cell length outcomes observed in $s k b 1$ mutant strains were determined to be due to a 
perturbation in cell cycle regulation (Gilbreth, et al., 1998). On the other hand, deletion of $h s l 7$ in S. cerevisiae leads to a slightly larger cell morphology and an elongated bud morphology, indicating that the absence of Hsl7 causes a delay in cell cycle progression resulting in an elongated bud formation (Shulewitz, et al., 1999). To explore this, the growth rates of $\Delta_{810-2832} h s l 7$ and $h s l 7^{\text {Otef }}$ strains were determined. Compared to the wild type progenitor strains, no difference in the rates of growth was observed in $h s l 7$ mutant strains (Table 5). In addition, localization of cell wall components was explored. Staining of both $\Delta_{810-2832} h s l 7$ and $h s l 7^{\text {Otef }}$ strains with CFW and WGA did not show any aberrations in cell wall material localization and deposition (Fig. 11B). This indicates that $\mathrm{Hs} 17$ does not appear to regulate growth rate, nor have any effect on localization and deposition of cell wall material in the cell.

Hsl7 of S. cerevisiae and Skb1 of S. pombe are involved in the response to environmental conditions (Fujita, et al., 1999; Bao, et al., 2001). This led me to investigate of the role $\mathrm{Hsl} 7$ of $U$. maydis might play in the response to environmental stimuli. Examination of $\Delta_{810-2832} h s l 7$ colonies on YEPS consistently showed small spikes protruding from the colonies, whereas the wild type progenitor strains showed smooth edges (Fig. 12A, see arrows). While different from the wild type progenitor strains, this phenotype was subtle and not indicative of a filamentous response. Strains over expressing $h s l 7$ displayed no differences in colony morphology compared to that of the wild type progenitor strains when grown on YEPS (Fig. 12A). Examination of YEPS micro-colonies at $20 \mathrm{x}$ magnification, $\Delta_{810-2832} h s l 7$ strains displayed a phenotype that could be described as "directional growth," where groups of cells parallel to one another run in a similar direction, different to that of another group of parallel cells (Fig. 12B, 

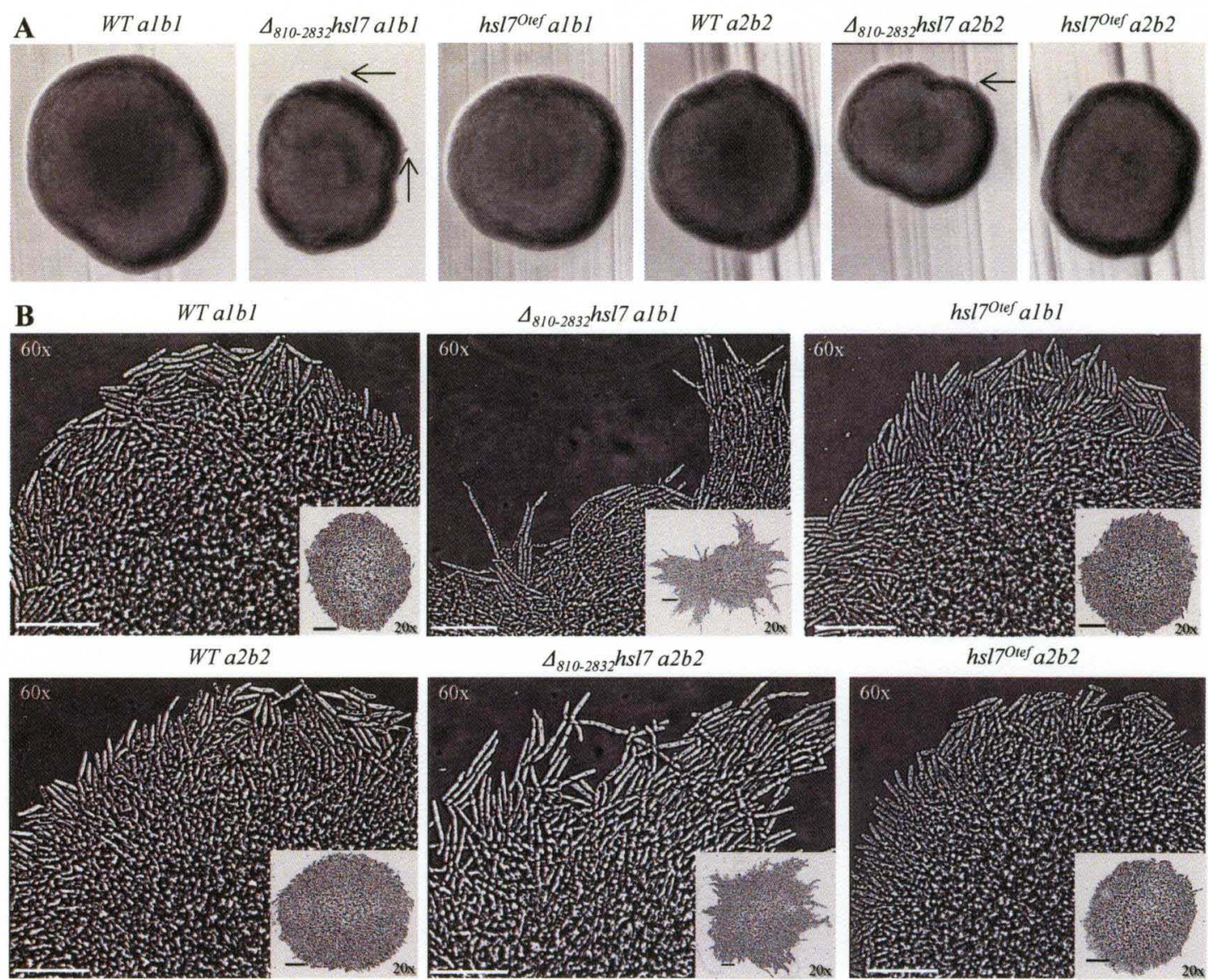

Figure 12: Disruption of $h s l 7$ leads to a directional growth phenotype, while overexpression of $h s l 7$ did not affect colony morphology on rich media. Grown on rich media, $\Delta_{810-2832} h s l 7$ colonies exhibit small protrusions of cells extending away from the colony edge. The colony morphology of $h s 7^{\text {otef }}$ mutant strains did not differ from that of the wild type strains, producing smooth colonies (A, see arrows). Examination of micro-colonies of $\Delta_{810-2832} h s l 7$ mutant strain indicated "directional growth" where groups of parallel cells grow in a specific direction different from another group of cells. $h s l^{\text {Otef }}$ mutant strains did not differ from the wild type strains (B). Scale bars, $50 \mu \mathrm{m}-20 \mathrm{x}$ inset image, $40 \mu \mathrm{m}-$ $60 \mathrm{x}$ image.

Inset). At 60x magnification, $\Delta_{810-2832} h s l 7$ strains displayed elongated cellular morphology similar to the increase in length observed in individual $\Delta_{810-2832} h s l 7$ cells (Fig. 12B). Also observed was a polarized growth pattern with several cells within a group growing in specific directions, different from group to group. Wild type progenitor cells did not display this directional growth phenotype, displaying unorganized distribution and arrangement of cells. This observation explains the small spikes seen in the colonies of the $\Delta_{810-2832} h s l 7$ strains as groups of parallel cells growing in a specific 
direction independent of other groups of cells. Overexpression of $h s l 7$ did not result in any observable phenotypic difference when the strains were grown on YEPS (Fig. 12B).

$\Delta_{810-2832} h s l 7$ strains grown on SLAD displayed an increase in the filamentous response, with longer filaments and a colony morphology that can be described as "spiney" (Fig. 13A). Overexpression of hsl7 did display a subtle decrease, but not

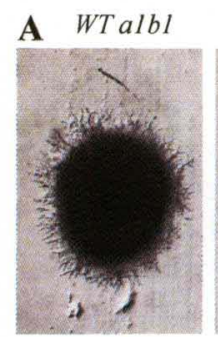

WT $a 2 b 2$

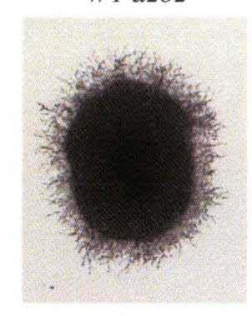

B

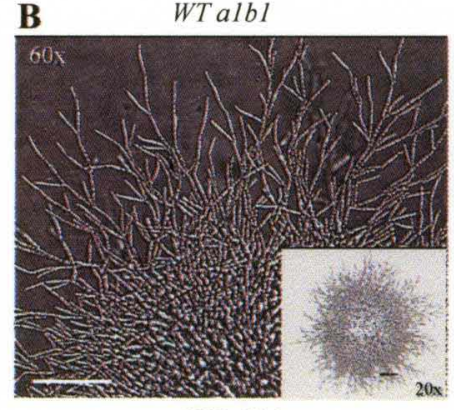

WT $a 2 b 2$

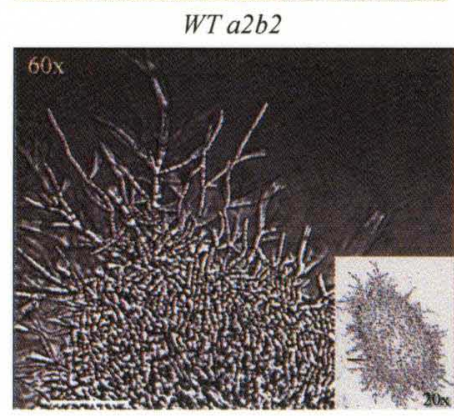

hsl7 Otef alb

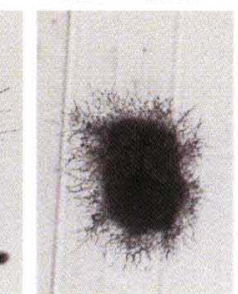

hsl7 Otef $a 2 b 2$

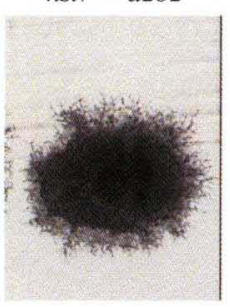

Figure 13: Filamentous response to low ammonium conditions of $\boldsymbol{h s l} 7$ mutants. $\Delta_{810}$ ${ }_{2832} \mathrm{hsl} / 7$ and $\mathrm{hsl} 7^{\text {Otef }}$ strains were grown on SLAD media and examined for filament formation. $\Delta_{810-}$ ${ }_{2832} \mathrm{hs} / 7$ colonies display an increase in the ability to form filaments while $h s 7^{\text {Otef }}$ colonies exhibit a decrease in filament formation, primarily in the a2b2 mating-type background (A). Microcolonies were utilized to examine filament formation at the cellular level. $\Delta_{810-2832} h s l 7$ strains were increased in filament length and branching, as well as displaying "directional growth." The $h s l 7^{\text {Otef }}$ strains displayed a decrease in both number and length of filaments (B). Scale bars, $50 \mu \mathrm{m}-20 \mathrm{x}$ inset image, $40 \mu \mathrm{m}-60 \mathrm{x}$ image.

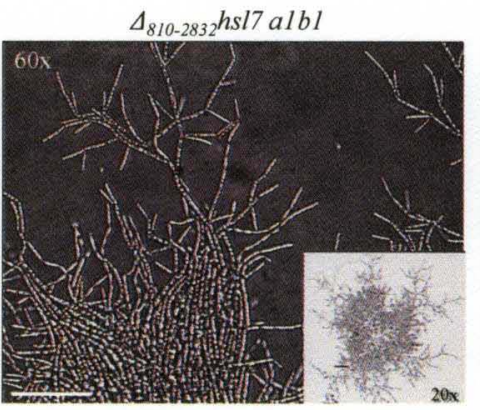

$\Delta_{810-2832} h s l 7$ a $2 b 2$

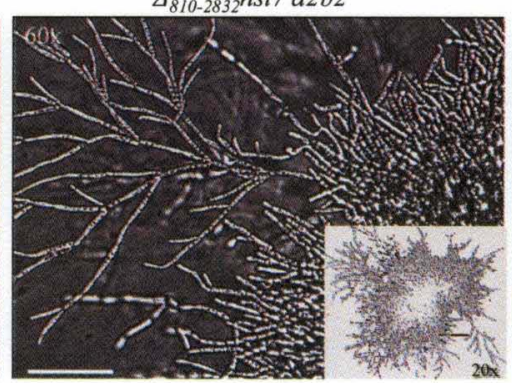

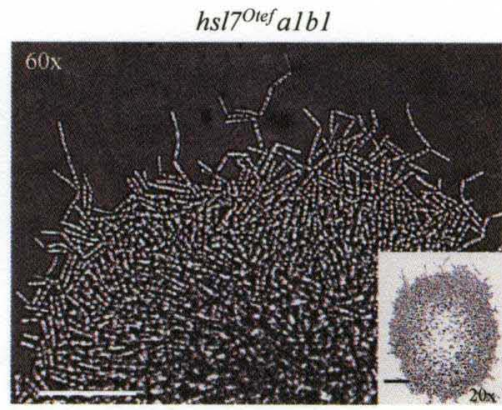

$h s l 7^{\text {Otef }} a 2 b 2$

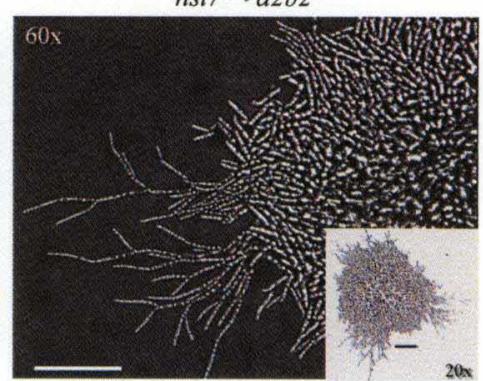

elimination, of the filamentous response to low ammonium conditions primarily in the

a2b2 mating background, indicating the $\mathrm{Hsl} 7$ plays a role in the filamentous response to 
low ammonium conditions in U. maydis (Fig. 13A). These results are similar to the phenotypes of $h s l 7$ mutants in response to low ammonium conditions in S. cerevisiae (Fujita, et al., 1999). $\Delta_{810-2832} 2 s l 7$ strains grown on SLAD micro-colonies produced the same directional growth phenotype observed for these strains on YEPS media when viewed at 20x magnification (Fig. 13B, Inset). At 60x magnification elongated highly branched filaments were evident. The filaments were longer and more highly branched than those observed in the wild type progenitor cells. The directional highly elongated filaments produced by $\Delta_{810-2832} h s l 7$ explain the observed "spiney" phenotype in colony morphology on SLAD media (Fig. 13B). A dramatic reduction in filament formation was observed for $h s l^{\text {Otef }}$ strains on SLAD when viewed at both 20x and 60x magnification (Fig. 13B). It appears that Smu1 and Hsl7 both play a role in cell elongation and the filamentous response to low ammonium conditions, but their effects tended to be opposite to one another. An increase in cell length and the filamentous response was observed in the $\Delta_{810-2832} h s l 7$ and $s m u l^{\text {Otef }}$ strains, while a decrease in cell length and a reduction the filamentous response on low ammonium media were seen in the $\Delta s m u l$ and $h s l 7^{\text {Otef }}$ strains, primarily in the $a 2 b 2$ strains (in cell length only for $a 2 b 2 h s l 7^{\text {Otef }}$ strains). In addition, the $s m u I^{\text {Otef }}$ colonies grown on SLAD did not display the directional growth phenotype observed in the $\Delta_{810-2832} h s l 7$ mutant background.

To determine if $\mathrm{Hsl} 7$ is involved in the production of filaments as a response to pheromone, plate mating assays were utilized. Assessment of mating efficiency relies on the production of white aerial hyphae on the black background of YPD-activated charcoal media when strains of opposite mating-type are mixed and spotted. Wild type and the $\Delta_{810-2832} h s l 7, h s l 7^{O t e f}$ mutant strains were mixed and spotted onto YPD-activated charcoal 
media. No differences in mating efficiencies were observed among these strains (Fig. 5). The mating assay indicates that Hsl7 does not appear to play a significant role in the mating response, ultimately having no effect on filament formation due to pheromone. Exploration of the cell wall stressors, CFW and CR, also yielded no discernable differences compared to the wild type progenitor strains (data not shown). Taken together, these results indicate that Hsl7 plays a role in the filamentous response pathway to low ammonium conditions, but not responses to pheromone or cell wall stressors.

\section{Disruption of smul in a $\Delta_{810-2832} h s / 7$ background does not rescue any of the} phenotypes associated with the $\Delta_{810-2832} h s / 7$ background. In order to determine if there is a genetic interaction between Hsl7 and Smul in vivo, double disruption mutants were created. In both the $a l b 1$ and the $a 2 b 2 \Delta_{810-2832} h s l 7$ backgrounds, a smul disruption construct was introduced by the same method previously described and this was confirmed by PCR (data not shown). The mutants were viable, demonstrating that the absence of both $\mathrm{Hsl} 7$ and Smul is not lethal. The disruption of $s m u l$ in the $\Delta_{810-2832} h s l 7$ background did not attenuate the elongated phenotype of the $\Delta_{810-2832} h s l 7$ strains (Fig. 14A and Table 3), nor did it have any effect on the rate of growth (Table 5). Staining of the cell walls of the double disruption strains with CFW and WGA did not reveal any defect in the localization and deposition of cell wall material (Fig. 14B). Examination of colony formation on YEPS indicated that disruption of smul displayed a similar morphology of spikes protruding from the colonies, as observed in the $\Delta_{810-2832} h s l 7$ strains colony (Fig. 15A) pointing to the directional growth phenotype observed in the $\Delta_{810-2832} h s l 7$ strains. Further examination of colony morphology on micro-colonies indicated that disruption of $s m u 1$ in the $\Delta_{810-2832} h s l 7$ background did not alleviate the 


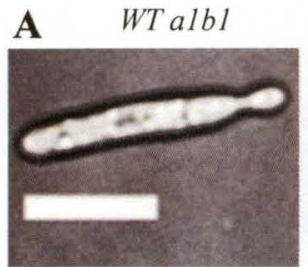

B
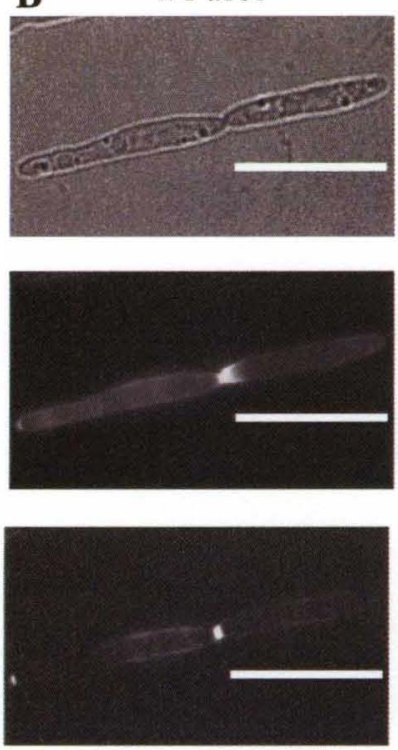

$\Delta_{810-2832} h s l 7 \Delta s m u l$ albl
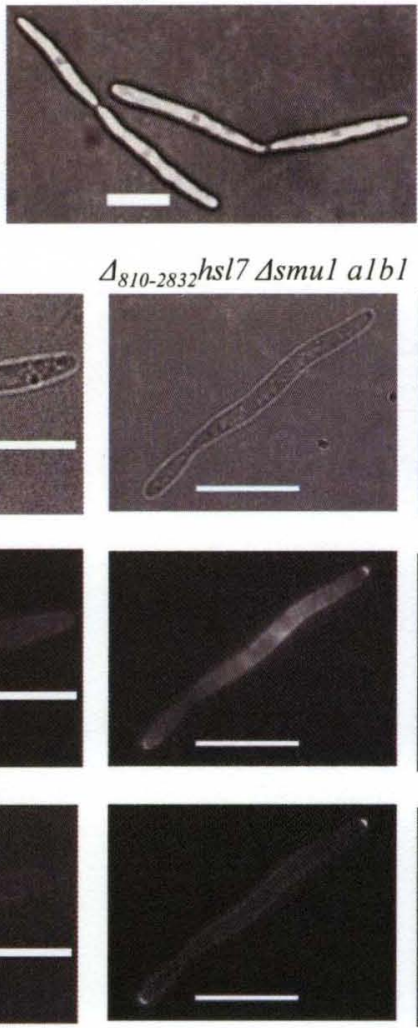

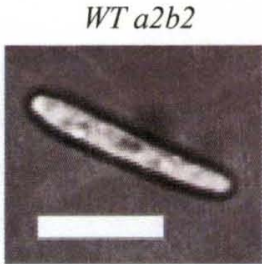

$W T a 2 b 2$
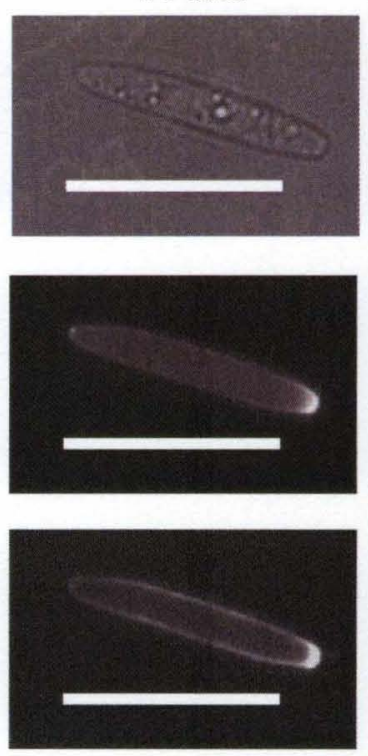

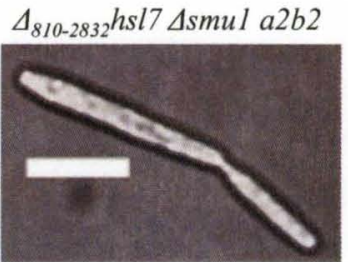

$\Delta_{810-2832} h s l 7$ ssmul albl
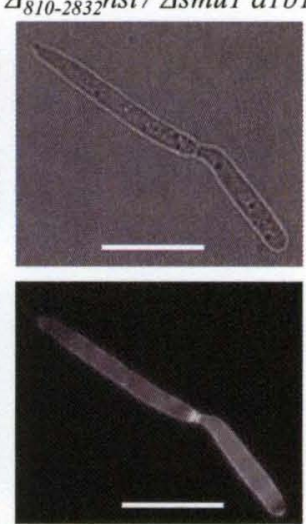

WGA

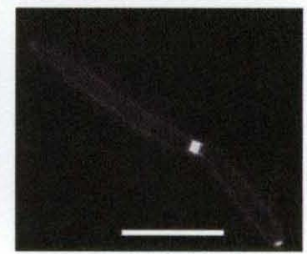

Figure 14: Disrupting smu1 in a $\Delta_{810-2832} h s / 7$ background does not rescue cell length increases observed in the $\Delta_{810-2832} h s l 7$ background, nor does it affect cell wall localization. $\Delta_{810-2832} h s l 7$ $\Delta s m u l$ cells display an increase in cell length comparable to $\Delta_{810-2832} h s l 7$ mutant strains (A). $\Delta_{810-2832} h s l 7$ $\Delta s m u l$ cells do not display any defects in cell wall localization (B). Scale bars, $10 \mu \mathrm{m}$.

directional growth phenotype observed previously (Fig. 15B). On SLAD, $\Delta_{810-2832} h s l 7$

$\triangle$ smul strains exhibited the same increase in the filamentous response to SLAD media (Fig. 16A) resulting in the "spiney" morphology seen in the $\Delta_{810-2832} h s l 7$ strains presented earlier. The increase in the filamentous response to low ammonium conditions was also observed on SLAD micro-colonies (Fig. 16B). In sum, disruption of smul did not rescue the colony morphology aberrations seen in the $\Delta_{810-2832} h s l 7$ strains. In addition, $\mathrm{CFW}$ or CR did not adversely affect the $\Delta_{810-2832} h s l 7 \Delta s m u l$ strains relative to the wild type strains. Moreover, disruption of $s m u l$ in the $\Delta_{810-2832} h s l 7$ background did not alleviate the mating type specific defects in the mating reaction observed in the smul disruption 

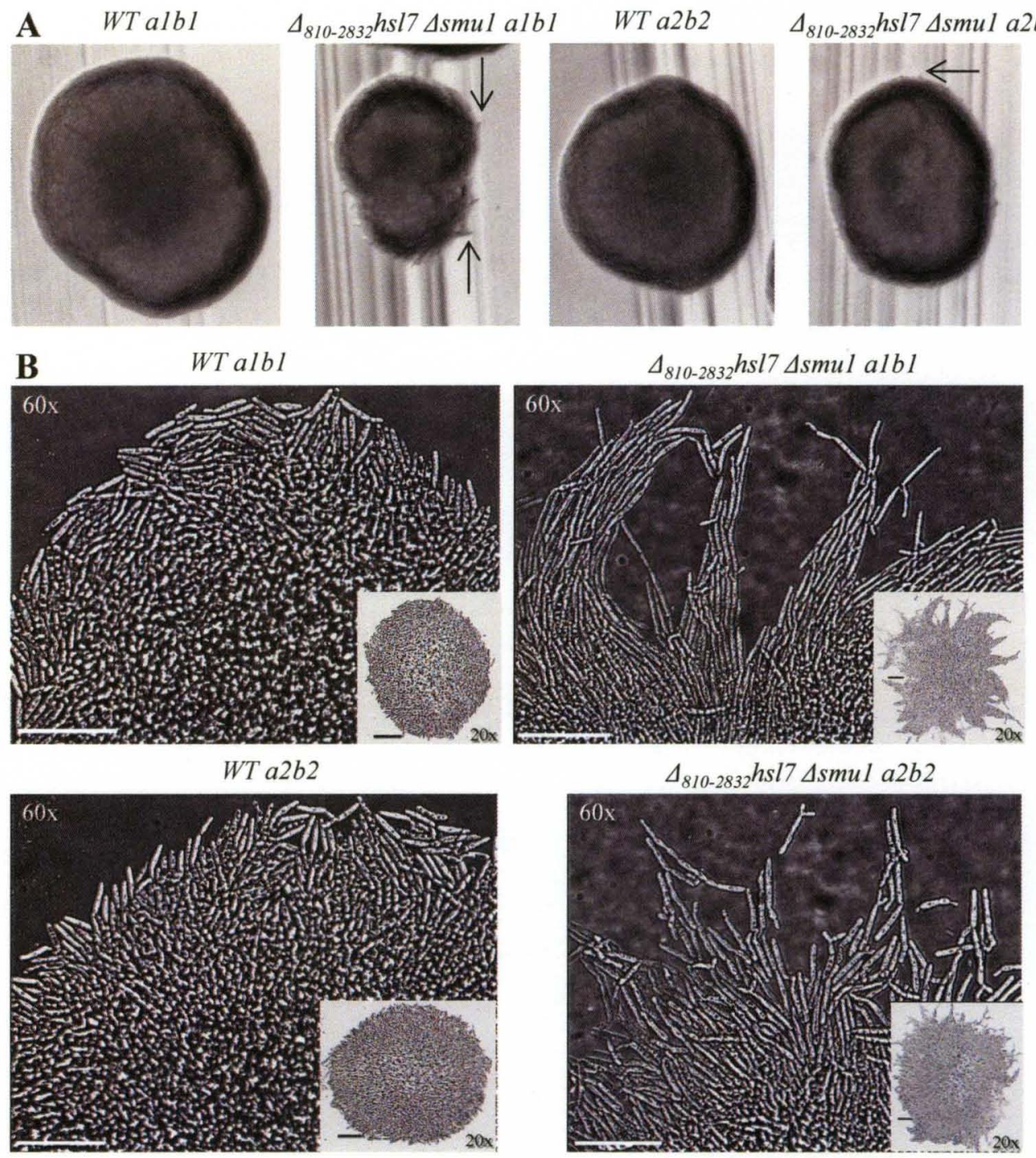

Figure 15: $\Delta_{810-2832} h s l 7 \Delta s m u 1$ strains produce the directional growth phenotype on rich media. Small protrusions were observed in the $\Delta_{810-2832} h s l 7 \Delta s m u l$ colonies (A, see arrows). Micro-colonies of $\Delta_{810-2832} h s l 7 \Delta s m u l$ displayed the "directional growth" phenotype of parallel groups of cells growing in a specific direction (B). Scale bars, $50 \mu \mathrm{m}-20 \mathrm{x}$ inset image, $40 \mu \mathrm{m}-60 \mathrm{x}$ image.

background (Fig. 5).

\section{Overexpression of $s m u l$ in a $\Delta_{810-2832} h s l 7$ background exacerbates phenotypes}

associated with the $\Delta_{810-2832} h s / 7$ background on rich media. To continue the

examination of a possible genetic interaction between $h s l 7$ and $s m u l, \Delta_{810-2832} h s l 7$

${ }_{s m u 1^{\text {otef }}}$ mutant strains were created by introducing the $s m u 1^{\text {Otef }}$ construct into the $\Delta_{810 \text { - }}$ $2832 h s l 7 a 1 b 1$ and $a 2 b 2$ background strains as previously described. Examination of cell 

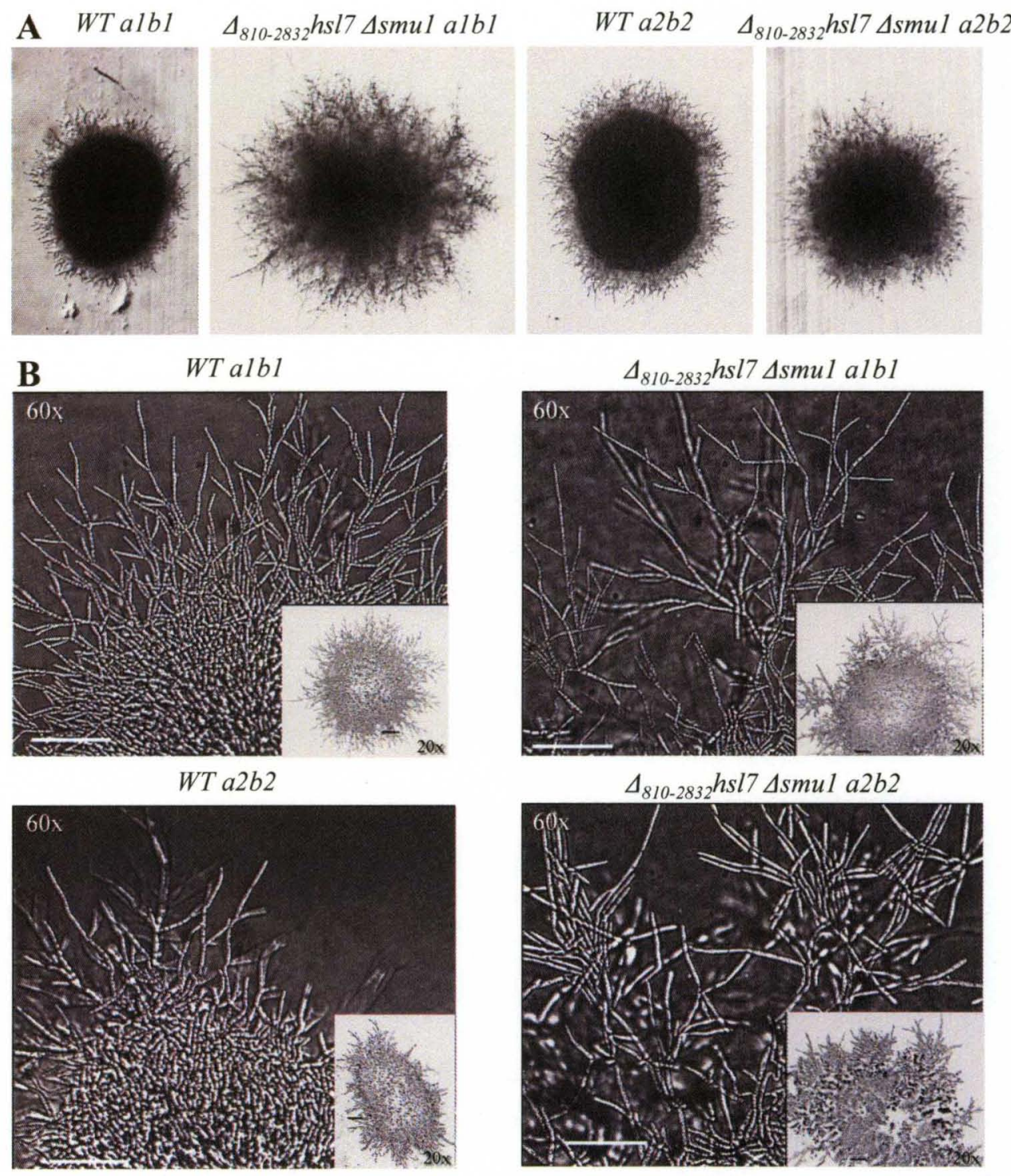

Figure 16: $\Delta_{810-2832} h s l 7 \Delta s m u 1$ mutants are hyper-filamentous on SLAD. On SLAD media, $\Delta_{810-2832} h s l 7 \Delta s m u l$ colonies display an increase in the ability to form filaments comparable to that of $\Delta_{810-2832} h s l 7$ colonies (A). Micro-colonies were utilized to examine filament formation at the cellular level. $\Delta_{810-2832} \mathrm{hs} / 7 . \Delta s m u 1$ strains were increased in filament length and branching, as well as displaying "directional growth." Thus, disruption of smul does not alleviate $\Delta_{810-2832} h s l 7$ associated phenotypes (B). Scale bars, $50 \mu \mathrm{m}-20 \mathrm{x}$ inset image; $40 \mu \mathrm{m}-60 \mathrm{x}$ image.

morphology indicated that $\Delta_{810-2832} h \mathrm{sl} 7 \mathrm{smul}{ }^{\text {Otef }}$ mutant strains were greatly increased in cell length when compared to the wild type progenitor strains (Fig. 17A and Table 3). Also when compared to $\Delta_{810-2832} h s l 7$ or $s m u l^{\text {Otef }}$ single mutant strains, the $\Delta_{810-2832} h s l 7$ $s m u 1^{\text {Otef }}$ mutant strains displayed increased cell lengths beyond either single mutant strain 

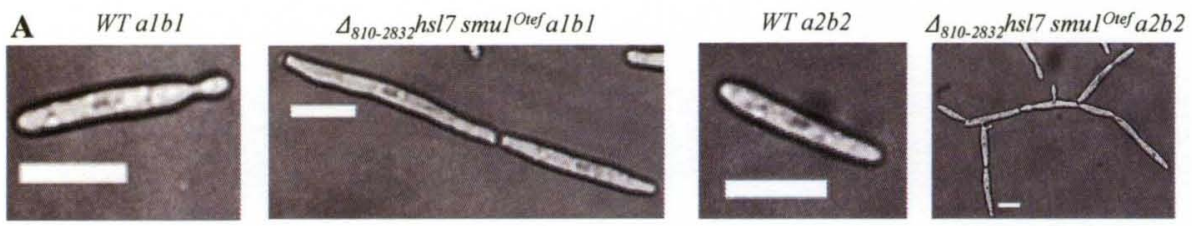

B

$\Delta_{810.2832} h s l 7 \mathrm{smu} 1^{\text {Otef }}$ albl

$\Delta_{810-2832} h s l 7$ smul ${ }^{\text {ref }}$ a $2 b 2$
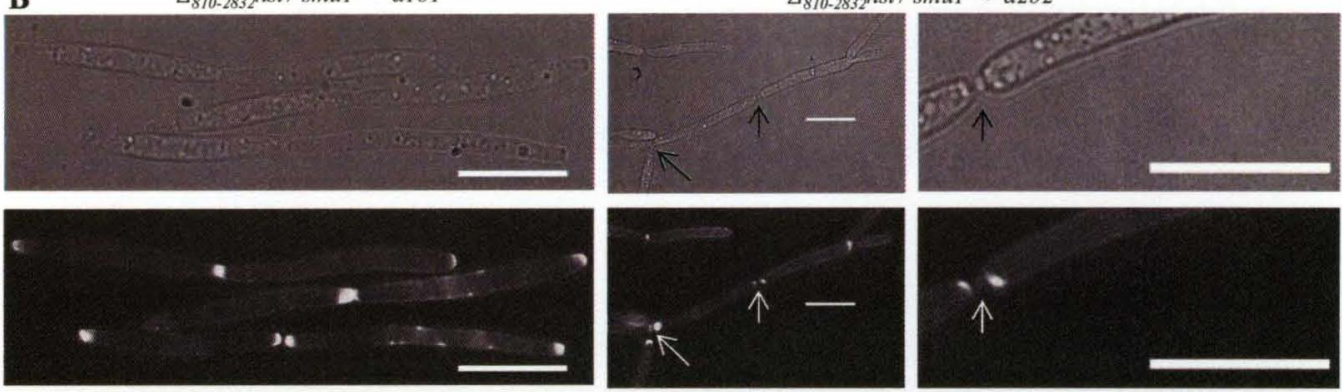

CFW
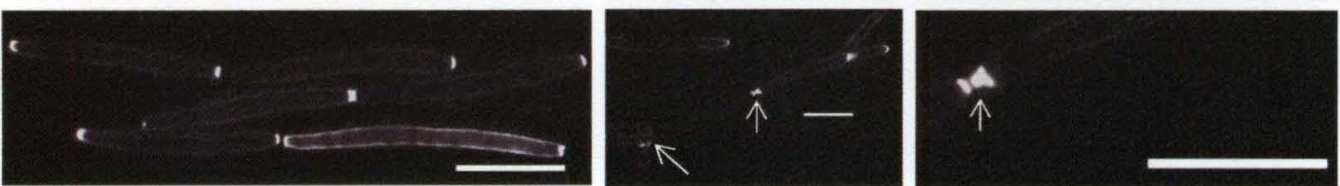

WGA

C

$\Delta_{810-2832}$ hsl7 smul ${ }^{\text {Otef }} a 2 b 2$
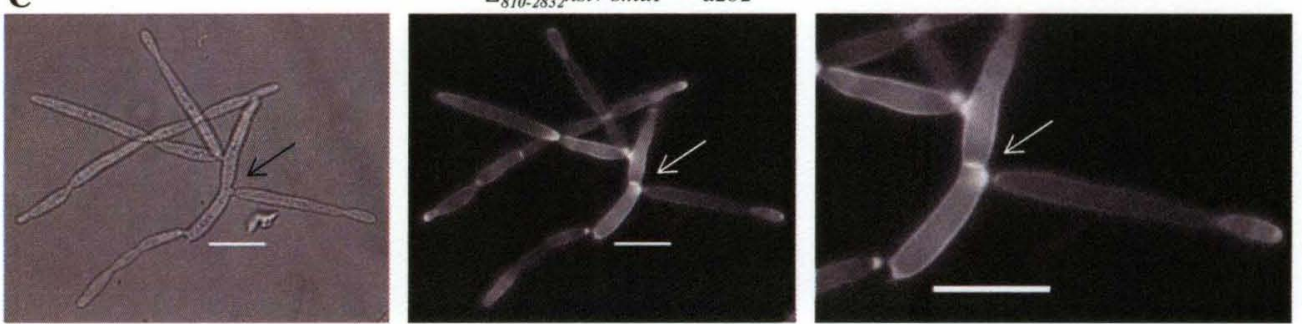

CFW

Figure 17: $\Delta_{810-2832} h s / 7 s m u 1^{\text {Otef }}$ cells are dramatically increased in cell length and the $a 2 b 2$ matingtype strain displays defects in cell separation. $\Delta_{810-2832} h s / 7 \mathrm{smu} 1^{\text {Otef }}$ cells display an increase in cell length above and beyond any previously examined strain. The $a 2 b 2$ mating-type strain exhibits cell separation defects (A). The $\Delta_{810-2832} h s l 7 s m u 1^{\text {Otef }}$ strains did not display any defects in cell wall localization with both mother and daughter cell septa properly formed, though the $a 2 b 2$ mating-type background cells did not correctly separate (B, see arrows). A small proportion of $\Delta_{810-2832} h s l 7 \mathrm{smu} 1^{\text {Otef }}$ cells displayed lateral budding that occurred at cross wall septa (C, see arrows). Scale bars, $10 \mu \mathrm{m}$.

(Table 6), yet the growth were similar to wild type strains (Table 5). In the $a 2 b 2$ background, the $\Delta_{810-2832} h s l 7$ smul ${ }^{\text {Otef }}$ mutant strain displayed mother daughter cell separation defects (Fig. 17A). Normal mother daughter cell separation in U. maydis is completed by the formation of two distinct septa, a primary septum in the mother cell and then, subsequently, a secondary septum in the daughter cell. The two septa outline a fragmentation zone that disintegrates, releasing the daughter cell from the mother cell

(Weinzierl, et al., 2002). To explore whether $\Delta_{810-2832}$ hsl7 smul ${ }^{\text {Otef }}$ mutant strains also 
TABLE 6. Cell length differences of the $A_{810-2832} h s / 7 \mathrm{smu} I^{\text {Otef }}$ strains.

\begin{tabular}{|c|c|c|c|c|}
\hline Strain & n & Length $^{A}$ (in $\mu \mathrm{m}$ ) & Comparison & $p^{B}$ \\
\hline$\Delta_{810-2832} h s l 7$ al & 167 & $25.22+/-.45$ & $\Delta_{810-2832} h s l 7$ al vs. $\Delta_{810-2832} h s l 7$ a2 & 0.45 \\
\hline smul ${ }^{\text {olef }}$ al & 308 & $21.75+/-.28$ & $\Delta_{810-2832} h s l 7$ al vs. $\Delta_{810-2832} h s l 7 \mathrm{smul}^{\text {olef }}$ al & $>.001$ \\
\hline$\Delta_{810-2832} h s l 7$ smul ${ }^{\text {Otef }}$ al & 143 & $27.86+/-.45$ & smul ${ }^{\text {Otef }}$ al vs. $\Delta_{810-2832} h s l 7$ smul otef al & $>.001$ \\
\hline$\Delta_{810-2832} h s l 7$ a2 & 406 & $24.78+/-.32$ & & \\
\hline$s m u I^{\text {otef }} a 2$ & 224 & $19.66+/-.30$ & $\Delta_{810-2832} h s l 7$ a2 vs. $\Delta_{810-2832} h s l 7 \mathrm{smul}$ Otef $_{\text {OL2 }}$ & $>.001$ \\
\hline$\Delta_{810-2832} h s l 7 s m u I^{\text {Otef }} a 2$ & 89 & $27.33+/-.86$ & smul ${ }^{\text {Otef }}$ a2 vs. $\Delta_{810-2832} h s l 7$ smul $I^{\text {Otef }}$ a2 & $>.001$ \\
\hline
\end{tabular}

N.S. = Not Significant

have deficiencies in septum formation, cell wall localization was examined with CFW and WGA. Neither $\Delta_{810-2832} h s l 7$ smul ${ }^{\text {Otef }}$ mutant strains showed any sign of cell wall aberrations, indicating that cell wall material is able to localize and deposit correctly to the growing tip and in the formation of the two distinct septa between mother daughter cells (Fig. 17B). In the $a 2 b 2 \Delta_{810-2832} h s l 7 s m u l^{\text {Otef }}$ mutant strain, staining identified both the primary and secondary septa, yet the cells remained connected (Fig. 17B, see arrows). However, a small subset of $a 2 b 2 \Delta 810-2832 h s l 7 s m u 1^{\text {Otef }}$ mutant cells produced cross wall septa, while failing to form the normal mother daughter separation septa (Fig. 17C, see arrows). Two potential explanations could be that the cells are either unable to synthesize or are unable to localize all the vesicular elements and membrane aggregates required for defragmentation between the mother daughter septa. A closer examination of the $a 2 b 2 \Delta_{810-2832} h s l 7 s m u l^{\text {Otef }}$ mutant strain also indicated the formation of lateral buds that appear to branch at a point where a cross wall is formed (Fig. 17C, see arrows).

To further explore the pseudohyphal phenotype observed in the $\Delta_{810-2832} h s l 7$ $s m u 1^{\text {Otef }}$ mutant strain (primarily in the $a 2 b 2$ background), two cell morphology aspects were examined: cell wall localization and number of nuclei. To this end, the cells were 
stained with WGA, CFW, and DAPI (nucleic acid stain - DNA specific). Staining with WGA and CFW again indicated no aberrations of the localization and deposition of cell wall material in the $\Delta_{810-2832} h s l 7$ smul ${ }^{\text {Otef }}$ mutant strains, yet the $a 2 b 2$ mutant strain failed to separate correctly, forming pseudohyphae, with random cross wall septa interspersed in the cells (Fig. 18). The observed cross walls were similar to a central septum,

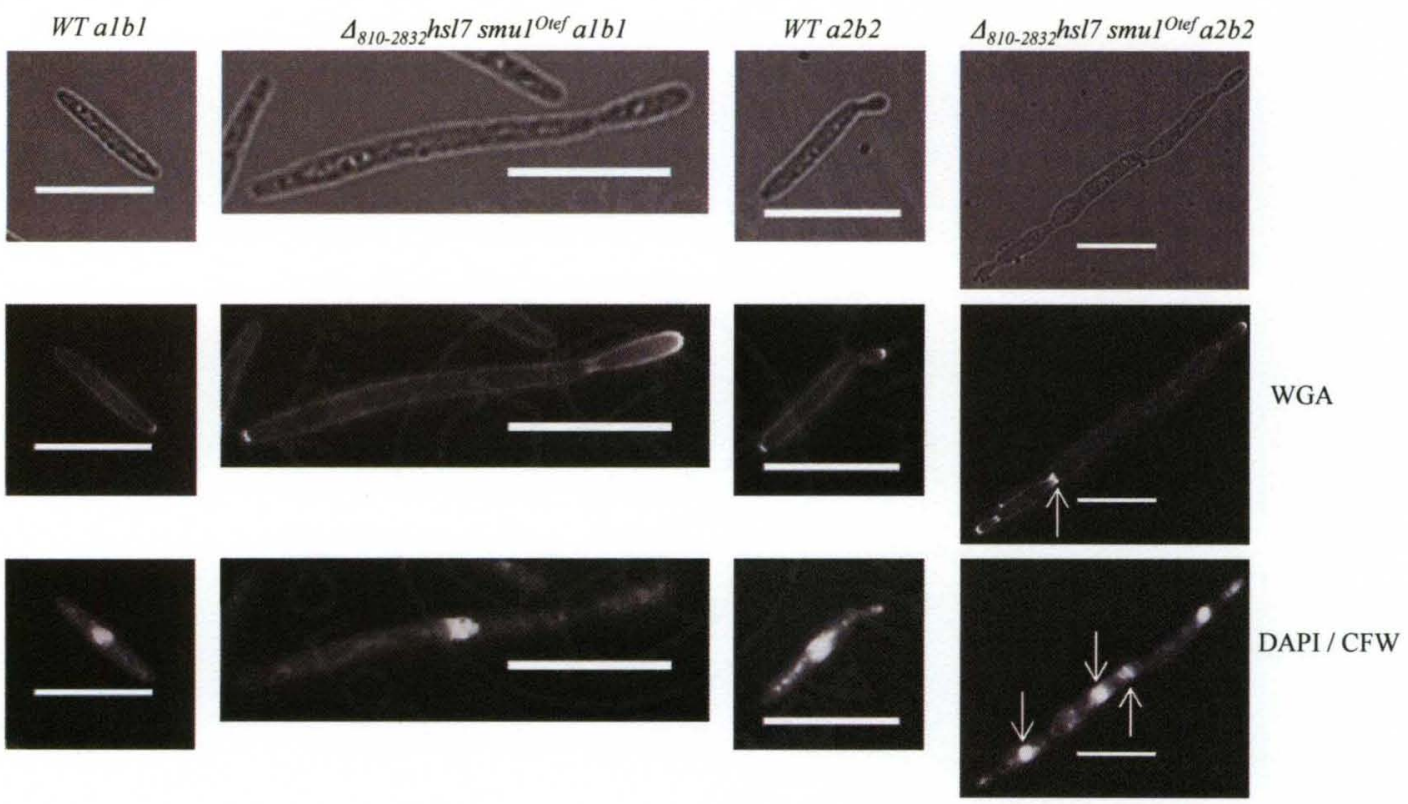

Figure 18: The $a 2 b 2$ mating-type $\Delta_{810-2832} h s l 7 s m u 1^{\text {Otef }}$ strain is multinucleic. albl $\Delta_{810-2832} h s l 7$ $s m u 1^{\text {Otef }}$ cells are elongated but display a single nucleus per cell. The a2b2 $\Delta_{810-2832} \mathrm{hsl}$ smul ${ }^{\text {otef }}$ cells exhibited a single nucleus per septated compartment (see arrows). Scale bars, $10 \mu \mathrm{m}$.

observed in the fission yeast, $S$. pombe (Sipiczki, 2007). Visualization of alb1 $\Delta_{810-}$ ${ }_{2832} \mathrm{hsl7}$ smul ${ }^{\text {Otef }}$ mutant cells also stained with DAPI exhibited a single bright spot representing the nucleus, comparable to the wild type progenitor strains (Fig. 18). When stained with DAPI, the $a 2 b 2 \Delta_{810-2832} h s l 7 s m u 1^{\text {Otef }}$ mutant strain displayed several connected cells, each with a single nucleus (Fig. 18, see arrows). This is different from what was observed in the wild type strains, where cells are separate from one another and contain a single nucleus. In addition, the pseudohyphal phenotype was only observed in the $a 2 b 2$ mating-type background, not in the $a l b 1 \Delta_{810-2832} h s l 7 s m u l^{\text {Otef }}$ mutant strain. 
To further explore the defect in mother daughter cell separation and lateral bud formation, colony and micro-colony morphologies on YEPS media were examined. The $\Delta_{810-2832} h s l 7 \mathrm{smul}{ }^{\text {otef }}$ mutant strains exhibited a filamentous colony morphology; this is drastically different than what is observed for the wild type progenitor strains or any of the other mutant strains previously tested (Fig. 19A). Micro-colony examination of $\Delta_{810-}$ ${ }_{2832} \mathrm{hs} / 7 \mathrm{smul}{ }^{\text {Otef }}$ mutant strains displayed a mating-type specific filamentous growth response at 20x magnification. The albl $\Delta_{810-2832} h s / 7 \mathrm{smul}{ }^{\text {Otef }}$ mutant strain displayed directional growth similar to the $\Delta_{810-2832} h s l 7$ mutant strains, however, the a2b2 $\Delta_{810 \text { - }}$ ${ }_{2832} \mathrm{hsl} 7 \mathrm{smul}{ }^{\text {Otef }}$ mutant strain exhibited filamentous growth (Fig. 19B, Inset). At 60x magnification, the differences between the $a 1 b 1$ and the $a 2 b 2$ mutant strains can be better observed. The $a 1 b 1 \Delta_{810-2832} h s l 7 \mathrm{smu1} 1^{\text {Otef }}$ mutant strain exhibited directional growth and pseudohyphal development of the cells making up the colony, although observations of the $a 2 b 2 \Delta_{810-2832} h s l 7 s m u I^{\text {Otef }}$ mutant strain displayed long single filaments and what appeared to be multiple areas of random cytoplasmic evacuation within each filament (Fig. 19B, see arrows). CFW staining of an $a 2 b 2 \Delta_{810-2832} 2 s l 7 s m u 1^{\text {Otef }}$ mutant strain micro-colony identified septated hyphae (Fig. 19C, see arrows).

\section{$\Delta_{810-2832} h s / 7$ smul ${ }^{\text {Otef }}$ mutant strains display a hyper-filamentous response to}

SLAD media. Observations of the $\Delta_{810-2832} h s l 7$ smul ${ }^{\text {Otef }}$ mutant strain colonies on SLAD media detected hyper-filamentous growth and again a more pronounced effect was seen in one of the two mating-types (Fig. 20A). The alb1 $\Delta_{810-2832} h s l 7 s m u 1^{\text {Oref }}$ mutant strain displayed the "spiney" morphology observed in the $\Delta_{810-2832} h s l 7$ mutant strains, whereas the $a 2 b 2 \Delta_{810-2832} h s l 7 s m u l^{\text {Otef }}$ mutant strain developed extremely long single filaments growing in a single direction from the filamentous edge of the colony. This phenotype, 

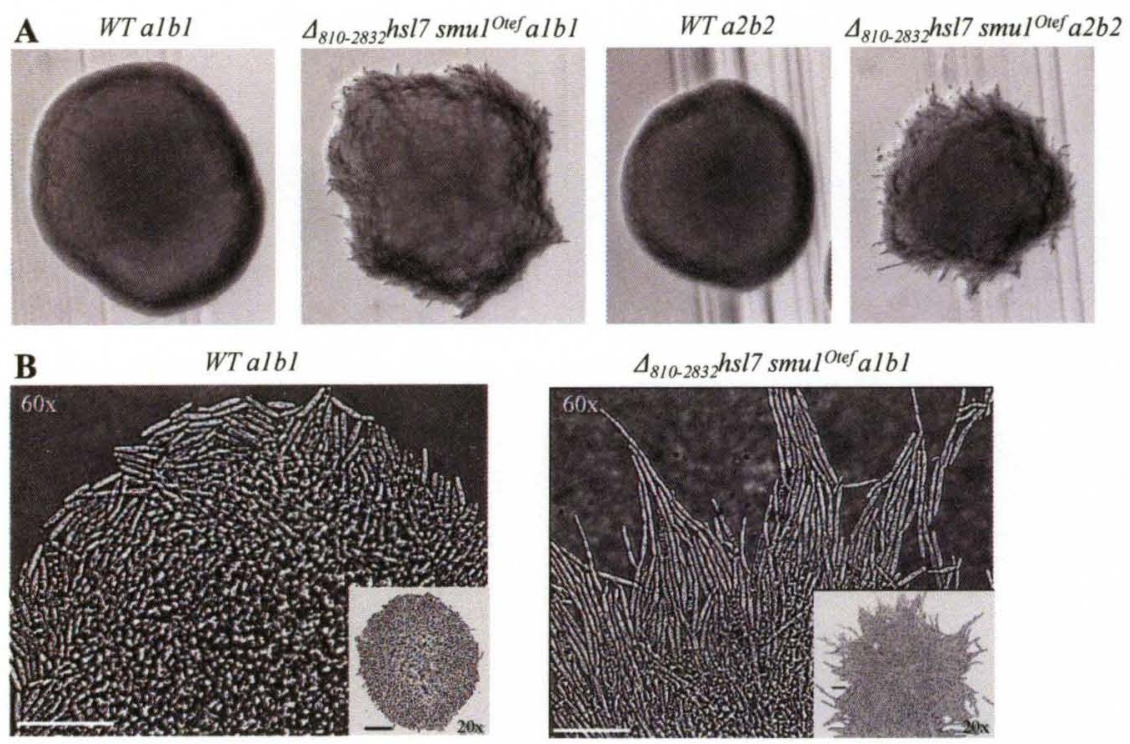

$W T a 2 b 2$

$\Delta_{810-2832}$ hsl7 smul ${ }^{\text {Otef }} a 2 b 2$
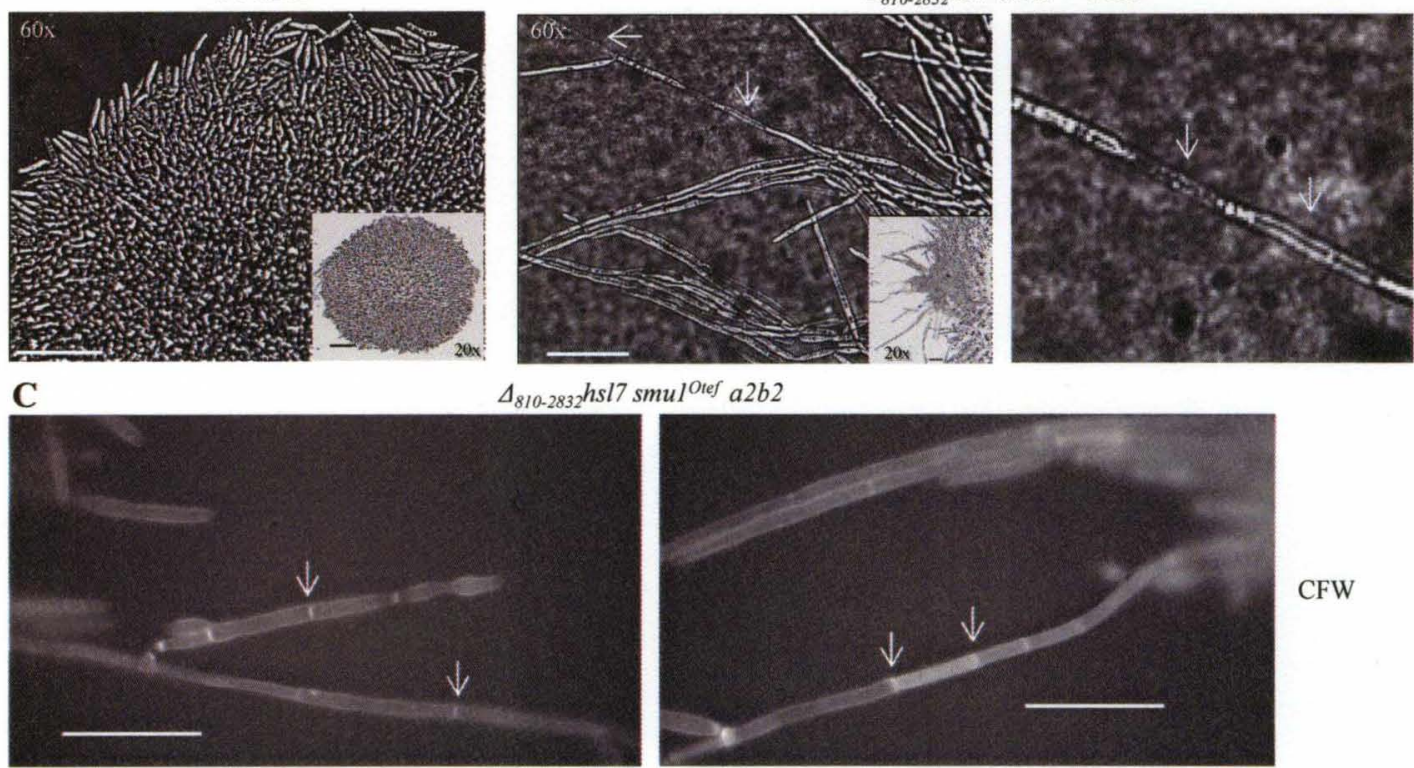

CFW

Figure 19: On rich media, the $\Delta_{810-2832} h s l 7 \mathrm{smul} \mathbf{S}^{\text {Otef }}$ strains are filamentous. $\left.\Delta_{810-2832} h s l 7 \mathrm{smul}\right|^{\text {otef }}$ strains produce filamentous colonies on rich media unlike wild type strains which are smooth in morphology (A). Micro-colonies of the $a 1 b 1 \Delta_{810-2832} h s l 7 s m u l^{\text {Olef }}$ strain displayed the "directional growth" phenotype while the cells were dramatically elongated. The albl $\Delta_{810-2832} h s l 7 \mathrm{smul}{ }^{\text {Otef }}$ strain exhibited filamentous growth with random areas of cytoplasmic evacuation within the filaments (B, see arrows). Scale bars, $50 \mu \mathrm{m}-20 \mathrm{x}$ inset image, $40 \mu \mathrm{m}-60 \mathrm{x}$ image. Staining of micro-colonies of the $a 2 b 2 \Delta_{810-2832} h_{s l 7} \mathrm{smul}^{\text {Otef }}$ strain with calcofluor white indicated that the filaments are septated hyphae (C, see arrows). Scale bars, $20 \mu \mathrm{m}$.

best described as a "starburst" phenotype, was not observed in any other mutant

previously examined. In addition, the single long filaments displayed in $a 2 b 2 \Delta_{810}$ ${ }_{2832} h s l 7$ smul ${ }^{\text {Otef }}$ mutant strain appear to have a darkened region at the end of each 

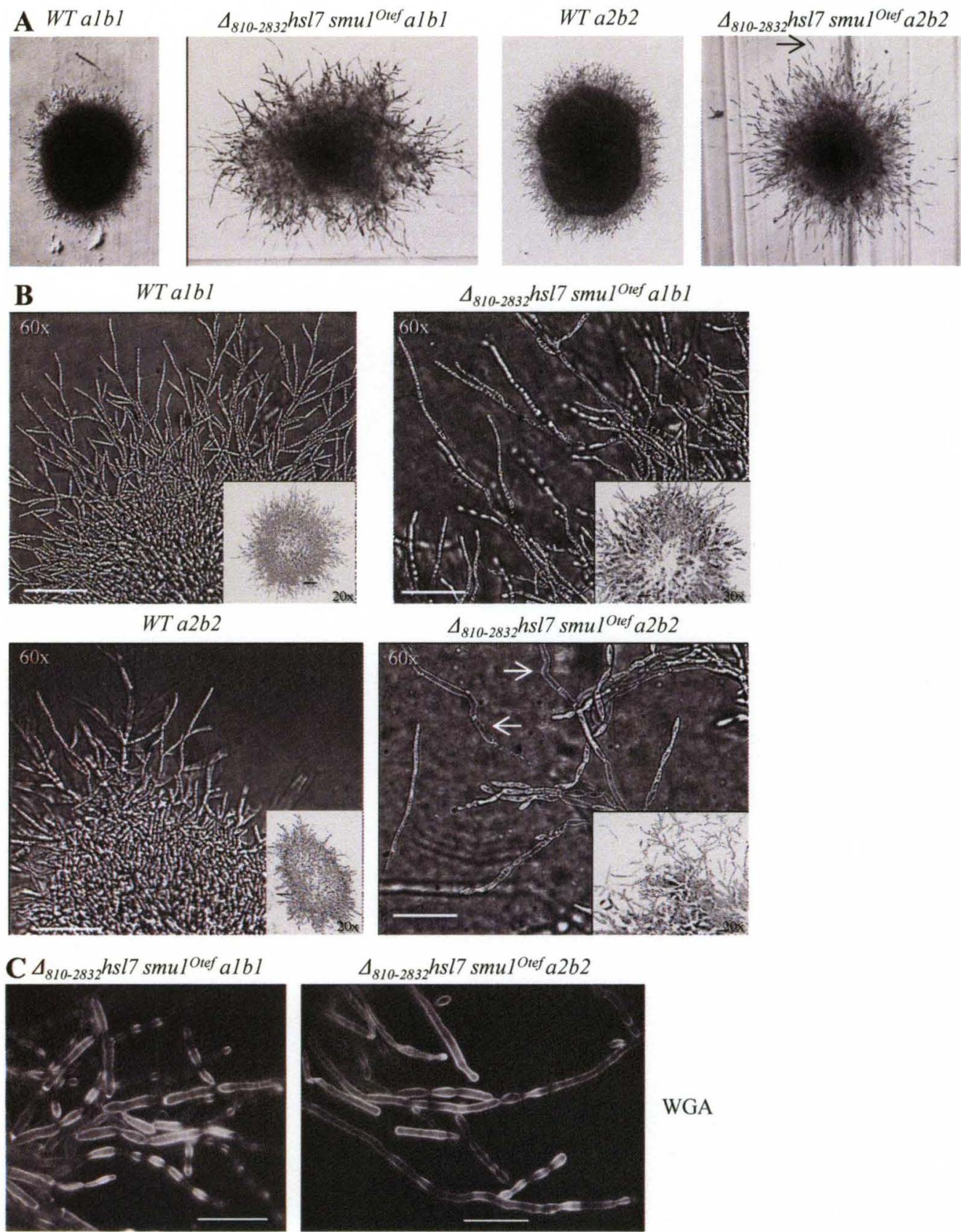

WGA

Figure 20: On SLAD media, the $\Delta_{810-2832} h s l 7 s^{s m u} 1^{\text {Otef }}$ strains are hyper-filamentous. $\Delta_{810-2832} h s l 7$ $s m u 1^{\text {Otef }}$ strains produce exaggerated filamentous colonies on SLAD media and are dramatically more filamentous than wild type strains. Also filaments of the $a 2 b 2 \Delta_{810-2832} h s l 7 \mathrm{smul}{ }^{\text {Otef }}$ strain exhibited darkened regions at the tips of empty filaments (A, see arrows). Micro-colonies of the $\Delta_{810-2832} h s l 7$ $s m u l^{\text {Otef }}$ strains displayed extremely elongated filaments and the $a 2 b 2 \Delta_{810-2832} h s l 7 s m u l^{\text {Otef }}$ strain exhibited random areas of cytoplasmic evacuation within the filaments (B, see arrows). Scale bars, 50 $\mu \mathrm{m}-20 \mathrm{x}$ inset image, $40 \mu \mathrm{m}-60 \mathrm{x}$ image. Staining of micro-colonies of the $\Delta_{810-2832} \mathrm{hsl} \mathrm{smul} \mathrm{s}^{\text {Olef }}$ strains with rhodamine-labeled WGA indicated chitin localization defects (C, see arrows). Scale bars, $20 \mu \mathrm{m}$. 
filament emerging from a clear empty filament (Fig. 20A see arrows). The cytoplasmic evacuation is similar, yet more extensive, than what is observed when the $a 2 b 2 \Delta 810$ ${ }_{2832} h s l 7$ smul ${ }^{\text {Otef }}$ mutant strain is grown on YEPS media. To get a closer examination of the effects of low ammonium conditions on these specific mutants, micro-colonies were again utilized. At $20 \mathrm{x}$ magnification, the expanse of filamentous growth of the $\Delta 810$ ${ }_{2832} h \mathrm{sl} 7 \mathrm{smul}{ }^{\text {Otef }}$ mutant strains can be seen (Fig. 20B, Inset). At 60x magnification, the albl $\Delta_{810-2832} h s l 7$ smul $^{\text {Otef }}$ mutant strain displayed long branched filaments and random cytoplasmic evacuation; moreover, the $a 2 b 2 \Delta_{810-2832} h s l 7$ smul ${ }^{\text {Otef }}$ mutant strain filaments were exceedingly long and branched compared to those of the albl and exhibited dramatic regions of cytoplasmic evacuation (Fig. 20B, see arrows). Staining of the micro-colonies of the $\Delta_{810-2832} h s l 7 s m u l^{\text {Otef }}$ mutant strains indicated chitin delocalization throughout the length of the filaments of both the $a l b l$ and $a 2 b 2$ mutant strains (Fig. 20C).

To explore cell wall deposition in the $\Delta_{810-2832} h s l 7$ smul ${ }^{\text {Otef }}$ mutant strains, the strains were grown in liquid SLAD. The strains were grown overnight (due to their slower growth in SLAD media than in YEPS media) and stained with CFW and WGA. Initial observations of wild type progenitor cells indicated that wild type cells do not filament in liquid SLAD (Fig. 21) as opposed to solid SLAD media. In addition, the previously described mutant strains grown in liquid SLAD also did not display filamentous growth, nor any aberrations in cell wall localization and deposition or number of nuclei (Fig. 22). However, the $\Delta_{810-2832} h s l 7$ smul ${ }^{\text {Otef }}$ mutant strains exhibited irregular bubbled hyphae morphology observed on solid SLAD media, but upon staining with CFW and WGA, very few cross walls or septa were observed (Fig. 21). Moreover, 

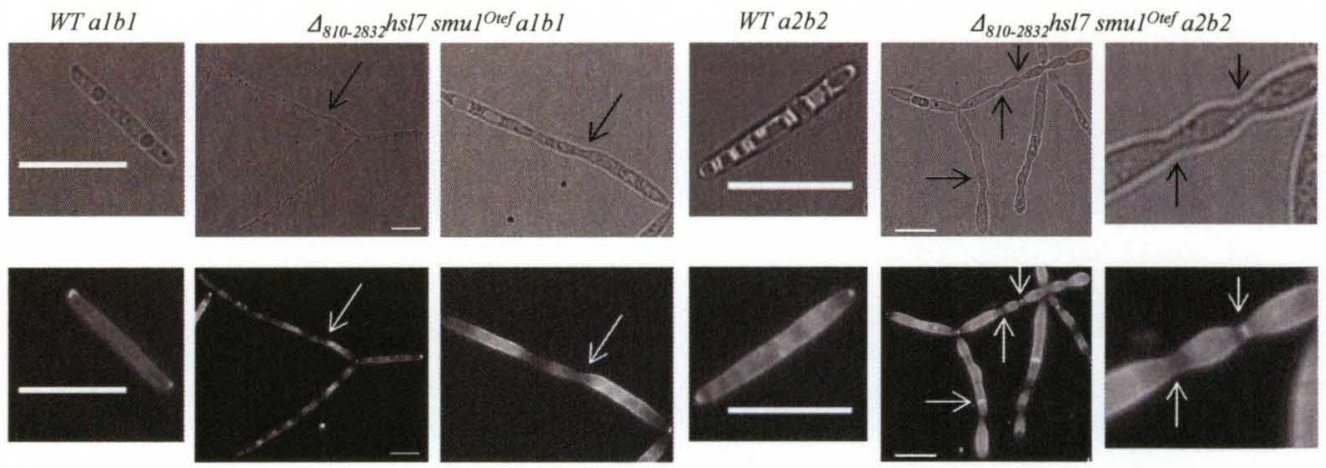

CFW
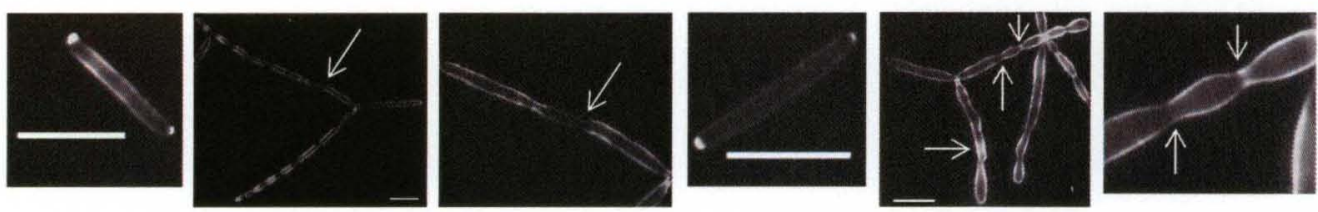

Figure 21: $\Delta_{810-2832} h s / 7 s m u I^{\text {Otef }}$ strains produce filaments in liquid SLAD and display defects in cell wall localization. $\Delta_{810-2832} h s l 7 \mathrm{smul}^{\text {Otef }}$ cells produce filaments in response to liquid SLAD while WT cells do not. $\Delta_{810-2832} \mathrm{hs} / 7 \mathrm{smul}{ }^{\text {Itef }}$ cells also exhibit a "bubbled" phenotype similar to pseudohyphae, yet at the narrowing of pseudohyphae, where a septum should form, very few septa appeared in the filaments (See arrows). Scale bars, $10 \mu \mathrm{m}$.

cell wall staining indicated that both chitin and the $\beta$-glucan were delocalized and unevenly distributed throughout the elongated cells. The bubbled hyphae appeared similar to pseudohyphae. Yet, where the missing septa should have formed in pseudohyphae, there was a decrease in the localization of cell wall material, while the few cross walls that were present, formed randomly within the bubbled hyphae (Fig. 21, see arrows). There was, however, a corresponding increase in the localization of cell wall material to the widest point of the bubble (Fig. 21). The only major difference observed between the mating-types of the $\Delta_{810-2832} \mathrm{hsl}$ smul ${ }^{\text {Otef }}$ mutant strains was greater bubbling appearance of the filaments from the $a 2 b 2$ mating-type background, compared to the alb1 $\Delta_{810-2832} h s l 7$ smul ${ }^{\text {Otef }}$ mutant strain (Fig. 21).

To determine if the $\Delta_{810-2832} h s l 7$ smul ${ }^{\text {Otef }}$ mutant strains are true hyphae or pseudohyphae, they were stained with WGA, CFW, and DAPI. The alb1 $\Delta_{810-2832} h s l 7$ $s m u 1^{\text {Otef }}$ mutant strain displayed an extremely long filamentous cell, containing no more 


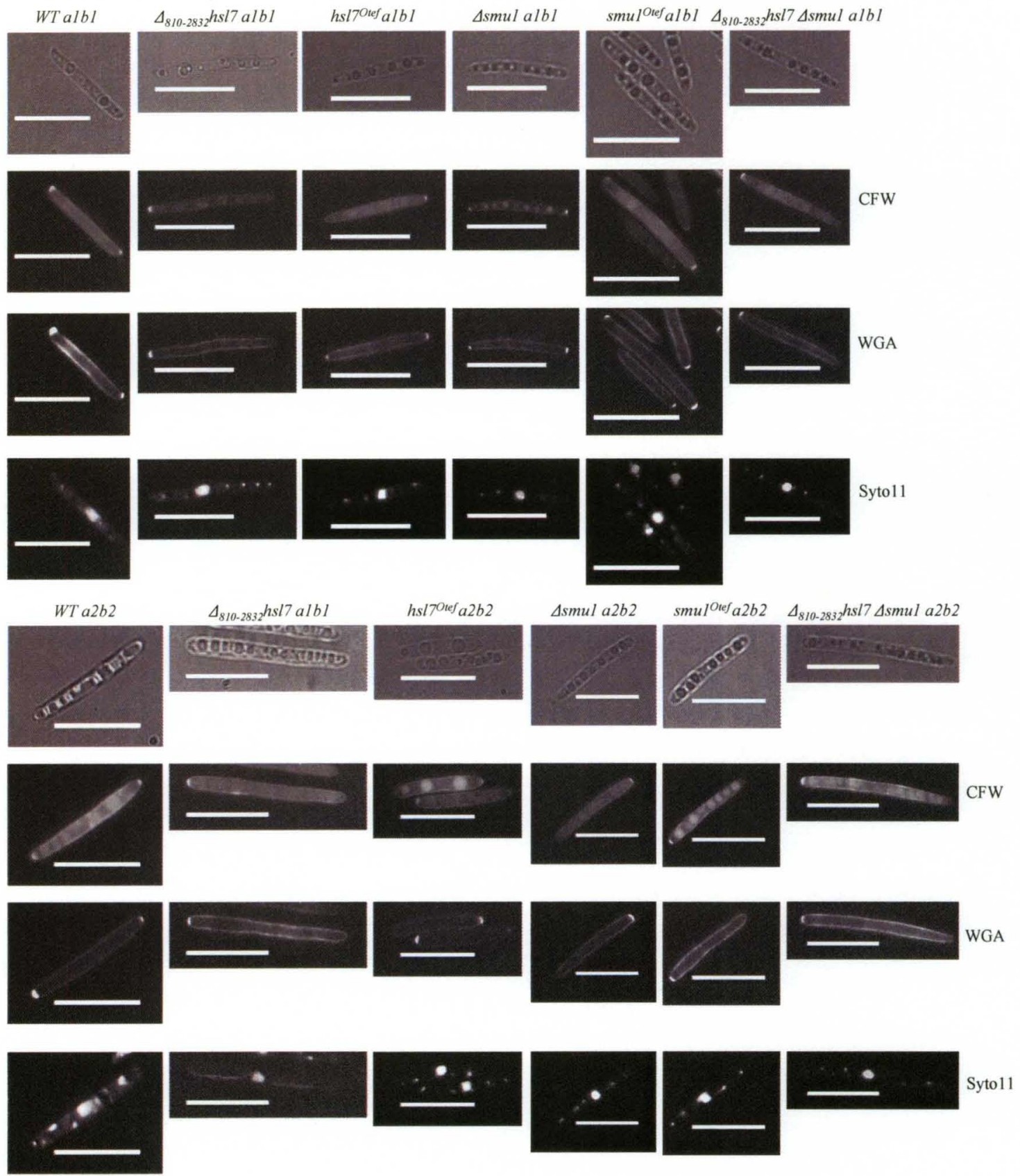

Figure 22: $\Delta_{810-2832} h s l 7, h s l^{0 t e f}, \Delta s m u 1, s m u 1^{O t e f}$, and $\Delta_{810-2832} h s l 7$ Asmu1strains were stained with CFW, WGA, and Syto 11. $\Delta_{810-2832} \mathrm{hsl} 7$, hsl $7^{0 t e f}, \Delta s m u l$, and $\Delta_{810-2832} h s l 7 \Delta s m u l$ strains do not display any defects in cell wall localization and nuclear content when grown in liquid low ammonium. Scale bars, $10 \mu \mathrm{m}$.

than one cross wall and a single brightly stained nucleus per filamentous section of the cell (Fig. 23A, see arrows). However, a2b2 $\Delta_{810-2832} h s l 7$ smul ${ }^{\text {Otef }}$ mutant strain exhibited multiple cells growing in a bi-polar fashion, separated by randomly interspersed cross 


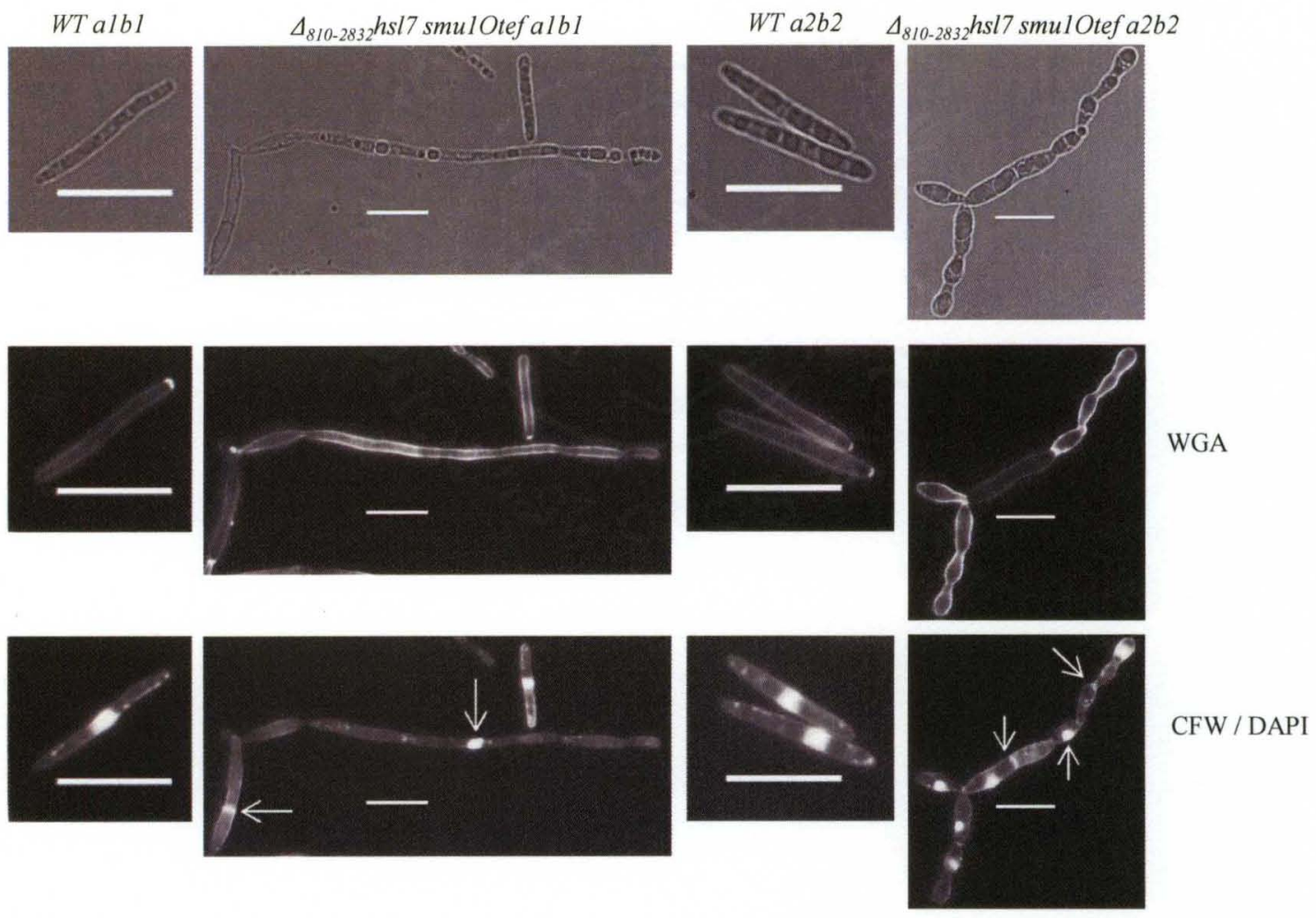

Figure 23: The mating-type specific effects of $\Delta_{810-2832} h s / 7$ smul ${ }^{\text {Otef }}$ strains in liquid SLAD. $a l b 1$ $\Delta_{810-2832} h s l 7$ smul $^{\text {Otef }}$ cells exhibit long filaments with a single, centrally located nucleus and septa (see arrows). The a2b2 $\Delta_{810-2832} h s / 7$ smul $1^{\text {Otef }}$ cells exhibited a "bubbled" hyphal phenotype with randomly spaced septa that separate the individual nuclei into compartments (see arrows). Scale bars, $10 \mu \mathrm{m}$.

wall septa. These cross walls do separate the multiple nuclei observed to a single nucleus per compartment. However, the cross walls were irregularly separated, with several forming in the middle of cell-like structures. Other septa were detected between two bubbled regions of the structure, similar to pseudohyphal cells where septa form between cells but the cells do not properly separate (Fig. 23B, see arrows). Overall these data lead to a conclusion that together Hsl7 and Smul play a role in the regulation of the filamentous response and cell separation in low ammonium conditions.

\section{$\Delta_{810-2832} h s l 7$ smul ${ }^{\text {Otef }}$ mutant strains are attenuated for virulence. To assess}

the roles of Hsl7 and Smul in virulence in U. maydis, identical compatible mutant strains of opposite mating-type were mixed and injected into maize seedlings ( 8 days post planting). Measures of virulence were taken at 7, 10, 14, 17, and 21 days post 
inoculation and three independent trials were performed. The cross of an $a 1 b l$ and an $a 2 b 2$ of a single mutant background were compared to a wild type progenitor cross. The mutant backgrounds examined were $\Delta_{810-2832} h s l 7, h s l 7^{\text {Otef }}, \Delta s m u l, s m u 1^{\text {otef }}, \Delta_{810-2832} h s l 7$ $\Delta s m u l$, and $\Delta_{810-2832} h s l 7 s m u l^{\text {Otef }}$. The virulence for each infection was measured by a disease index, ranking the severity of the disease symptoms on a scale from 0 to 5 . The results of a Kruskal-Wallis ANOVA with a Dunn's Multiple Comparisons Test, shown in Table 7, indicate that although slightly decreased for virulence compared to the wild type progenitor cross, $\Delta_{810-2832} h s l 7, h s l 7^{\text {Otef }}$, and $s m u 1^{\text {otef }}$ did not show a statistical difference compared to the wild type controls. The results are also presented in a percent of symptom formation graph (Doehlemann, et al., 2009; see Fig. 24). The $\Delta_{810-2832} h s l$, $h s l 7^{\text {Otef }}$, and $s m u l^{\text {Otef }}$ mutant strains all displayed a similar reduction in plant death (black shade on graph, Fig. 24) as well as an increase in symptom-free plants (Green shade on

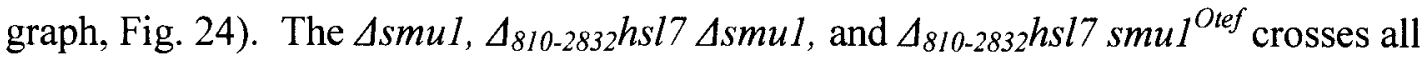
exhibited various decreases in virulence and they were all statistically different to the wild type controls in Kruskal-Wallis ANOVA with a Dunn's Multiple Comparisons Test $(\mathrm{p}<.001)$. In addition, the $\Delta s m u 1, \Delta_{810-2832} h s l 7 \Delta s m u 1$, and $\Delta_{810-2832} h s l 7 s m u 1^{\text {Otef }}$ crosses displayed a decrease in plant death and an increase in plants with a 0 or a 1 on the disease index scale (Fig. 24). The Dunn's Multiple Comparisons Test indicated that there was no significant difference in the reduction of virulence between the $\Delta s m u 1$ and $\Delta_{810-2832} h s l 7$ $\Delta s m u l$ mutant strains. The Dunn's Multiple Comparisons Test also indicated that the final mutant strain, $\Delta_{810-2832} h s l 7 \mathrm{smu} 1^{\text {Otef }}$, was significantly less virulent than all other compared strains (Table 7).

In addition to the measures of virulence taken at $21 \mathrm{dpi}$, virulence measures were 
TABLE 7. Pathogenicity of mutant strains ${ }^{A}$.

\begin{tabular}{|c|c|c|c|c|c|c|c|c|c|}
\hline \multirow[t]{2}{*}{ Treatment $^{\mathrm{B}}$} & \multirow[t]{2}{*}{ Strains } & \multirow{2}{*}{$\begin{array}{l}\text { Plants } \\
\text { Tested }\end{array}$} & \multicolumn{6}{|c|}{ Disease rating ${ }^{C}$ by number of affected plants } & \multirow{2}{*}{$\begin{array}{l}\text { Disease } \\
\text { Index }^{D}\end{array}$} \\
\hline & & & $\begin{array}{c}0 \\
\text { Points } \\
\end{array}$ & $\begin{array}{c}1 \\
\text { Point }\end{array}$ & $\begin{array}{c}2 \\
\text { Points }\end{array}$ & $\begin{array}{c}3 \\
\text { Points } \\
\end{array}$ & $\begin{array}{c}4 \\
\text { Points }\end{array}$ & $\begin{array}{c}5 \\
\text { Points }\end{array}$ & \\
\hline 1 & $W T$ & 58 & 1 & 5 & 3 & 1 & 12 & 36 & 4.17 \\
\hline 2 & $\Delta_{810-2832} h s l 7$ & 59 & 3 & 8 & 4 & 4 & 12 & 28 & 3.66 \\
\hline 3 & $h s l 7^{\text {Otef }}$ & 59 & 4 & 9 & 7 & 0 & 14 & 25 & 3.46 \\
\hline 4 & $\Delta s m u l$ & 59 & 4 & 8 & 17 & 5 & 14 & 11 & $2.85^{* * *}$ \\
\hline 5 & smul $I^{\text {Otef }}$ & 59 & 4 & 6 & 3 & 4 & 15 & 27 & 3.71 \\
\hline 6 & $\Delta_{810-2832} h s l 7 \Delta s m u l$ & 59 & 10 & 13 & 12 & 4 & 11 & 9 & $2.34^{* * *}$ \\
\hline 7 & $\Delta_{810-2832} h s l 7 \mathrm{smul} \mathrm{I}^{\text {Otef }}$ & 59 & 12 & 29 & 10 & 0 & 4 & 4 & $1.44^{* * *}$ \\
\hline
\end{tabular}

${ }^{A}$ Table shows combined data from three identical experiments of 19 to 20 plants each reported for 21 days postinoculation.

B Treatment consisted of the inoculation of $10^{5}$ cells $\mathrm{ml}^{-1}$ for each of the strains.

c The disease rating is measured on a scale of 0 to 5 points based on the severity of symptoms as follows: 0 points, no disease symptoms; 1 point, chlorosis / anthocyanin production; 2 points, small leaf galls; 3 points, small galls on stems; 4 points, large stem galls; 5 points, plant death.

D The disease index is calculated as the sum of the disease ratings divided by the number of plants tested and statistical analysis was performed using a Kruskal-Wallis ANOVA with a Dunn's Multiple Comparison Test from three independent experiments.

*** $p$ value less than .001 .

taken at 7, 10, 14, and $17 \mathrm{dpi}$ and disease indices for each time measure were calculated. The change in pathogenicity was calculated as the slope of a linear regression and was compared to the slope of the wild type strains. The $\Delta_{810-2832} h s l 7, h s l 7^{O t e f}$, and $s m u l^{\text {Otef }}$ strains did not show a statistical difference compared to the wild type controls. The $\Delta s m u l, \Delta_{810-2832} h s l 7 \Delta s m u l$, and $\Delta_{810-2832} h s l 7 s m u l^{\text {Otef }}$ crosses all exhibited significant decreases compared to the wild type controls (Table 8). In addition, there was no significant difference between $\Delta s m u l$ and $\Delta_{810-2832} h s l 7 \Delta s m u l$. The decrease slope of the $\Delta_{810-2832} h s l 7 s m u l^{\text {Otef }}$ was significant compared to both the $\Delta s m u l$ and $\Delta_{810-2832} h s l 7 \Delta s m u l$ 


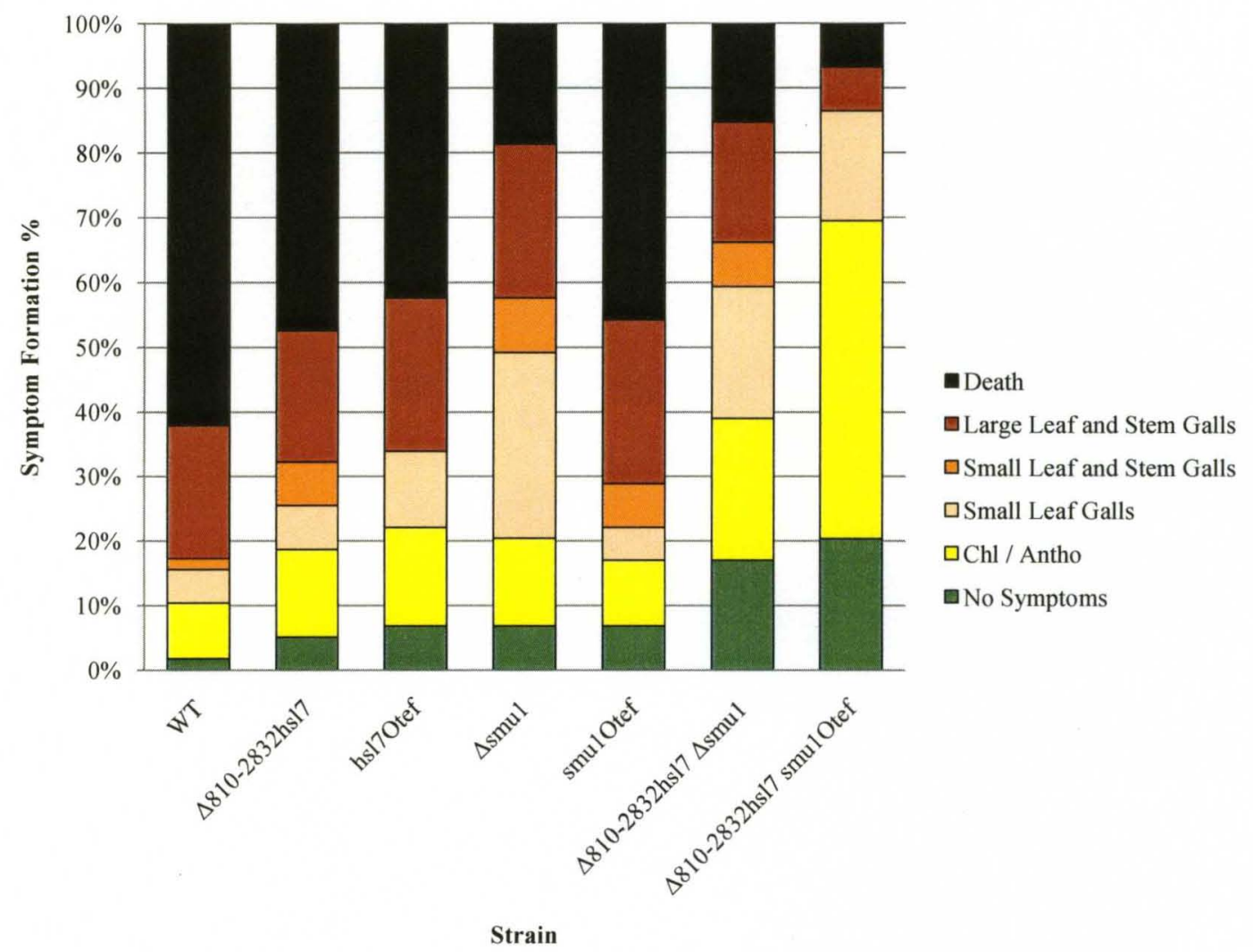

Figure 24: Disease symptom formation in various $U$. maydis mutant strains. All strains were rated at $21 \mathrm{dpi}$ and represent three independent experiments. Plants were inoculated with one paired

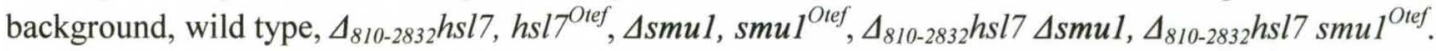
The graph displays the percentage of plants with a specific symptom of infections. Both $\Delta s m u 1$ and $\Delta_{810-}$ ${ }_{2832} h s l 7 \Delta s m u l$ exhibited a decrease in the percentage of dead plants, while the $\Delta_{810-2832} h s l 7 s m u l^{\text {Otef }}$ displayed a decrease of disease severity across all plants.

strains. These results point to an interesting interaction between Hsl7 and Smul in the ability to cause disease.

\section{DISCUSSION}

\section{Smu1 affects cell length and the filamentous response to low ammonium}

conditions. Previous work identified the Ste 20 homologue Smu1 and its roles in mating and pathogenicity (Smith, et al., 2004). In addition, disruption of smul decreases cell length, though only significantly in the $a 2 b 2$, and the filamentous response to low ammonium conditions. Conversely, ectopic overexpression of smul under the constitutively active Otef promoter displayed a significant increase in cell length in the 
TABLE 8. Changes in the rate of infection. ${ }^{A}$

\begin{tabular}{|c|c|c|c|}
\hline Strains $^{\mathrm{B}}$ & Slope ${ }^{C}$ & & $p^{D}$ \\
\hline$W T$ & $0.20 \pm 0.023$ & & \\
\hline & $0.18 \pm 0.022$ & $W T$ v. $\Delta_{810-2832} h s l 7$ & N.S. \\
\hline smul $1^{\text {Otef }}$ & $0.17 \pm 0.017$ & $W T$ v. hsl $7^{\text {Otef }}$ & N.S. \\
\hline$\Delta_{810-2832} h s l 7$ & $0.12 \pm 0.008$ & $W T$ v. $\Delta s m u l$ & $>.05$ \\
\hline$h s l 7^{O t e f}$ & $0.18 \pm 0.012$ & $W T$ v. $s m u l^{\text {Olef }}$ & N.S. \\
\hline$\Delta_{810-2832} h s l 7 \Delta s m u 1$ & $0.09 \pm 0.006$ & $W T$ v. $\Delta_{810-2832} h s l 7 \Delta s m u l$ & $>.05$ \\
\hline \multirow[t]{4}{*}{$\Delta_{810-2832} h s l 7 s m u l^{\text {Otef }}$} & $0.06 \pm 0.009$ & $W T$ v. $\Delta_{810-2832} h s l 7 s m u I^{O t e f}$ & $>.05$ \\
\hline & & $\Delta s m u l$ v. $\Delta_{810-2832} h s l 7 \Delta s m u l$ & $>.05$ \\
\hline & & $\Delta s m u l$ v. $\Delta_{810-2832} h s l 7 s m u I^{\text {Otef }}$ & $>.05$ \\
\hline & & $\Delta_{810-2832} h s l 7 \Delta s m u l$ v. $\Delta_{810-2832} h s l 7 s m u I^{\text {Otef }}$ & $>.05$ \\
\hline
\end{tabular}

A Table shows combined data from three identical experiments of 19 to 20 plants each reported for $7,10,14,17$, and 21 days postinoculation.

B Strains were mixtures of opposite mating background and co-inoculated.

C Disease index was calculated for each time point and used as a measure of pathogenicity. A slope for each mutant strain was calcuated from a linear regression and compared with the slope of the wild type strain.

D Slopes of regression were compared using Graphpad Prism 5.02.

N.S. $=$ Not Significant

albl mating-type background only. Growth rate analysis did not indicate any differences in either of the smul mutant strains; however, growth analysis may have not picked up subtle variations in the cell cycle of the mutant strains. Another possibility is that the cell length perturbations may not be due to cell cycle, but due to cytoskeletal defects potentially in actin or microtubule dynamics and polarization (Holly and Blumer, 1999; Qyang, et al., 2002). It is interesting to think that Smul may play a role in cytoskeletal organization, as well as regulating mating pheromone expression. Work in both $S$. cerevisiae and $S$. pombe suggests that Ste 20 homologues are involved in both the MAPK pathway and cell polarity through actin organization (Roberts and Fink, 1994; Marcus, et 
al., 1995; Gilbreth, et al., 1996 and 1998; Leberer, et al., 1997; Eby, et al., 1998; Holly and Blumer, 1999; Fujita, et al., 1999; Bao, et al., 2001; Qyang, et al., 2002). Evidence also suggests that Ste20 also plays a role in mitotic exit (Höfken and Schiebel, 2002). In addition, staining of the cell wall components, $\beta$-glucan and chitin, with CFW and fluorescently labeled WGA, respectively, did not indicate any defects in cell wall localization and deposition. Smul also displayed a subtle role in the filamentous response pathway to low ammonium conditions indicating Smu1 plays a role in the filamentous response pathway. And, this decrease was mating-type specific, occurring primarily in the $a 2 b 2$ mating-type background. Overexpression of $s m u l$ resulted in an increase in the filamentous response to SLAD media, which was confined to the $a l b 1$ mating-type specific background. Ste20-like kinases have been demonstrated to be involved in responses to environmental conditions regulating the MAPK pathway (Lengeler, et al., 2000; Bao, et al., 2001). Smulcould potentially regulate the filamentous response to low ammonium conditions via activation of the MAPK pathway; however this has yet to be established.

\section{Hs17 negatively affects cell length and the filamentous response to SLAD.}

Studies of the Ste20 homologues from S. cerevisiae and S. pombe indicated that the respective Hs17 homologues interact with the PAK kinases both in vitro and in vivo (Fujita, et al., 1999; Gilbreth, et al., 1996 and 1998; Bao, et al., 2001). Here I identified Hsl7, a sole protein-arginine methyltransferase homolog in U. maydis. Haploid $U$. maydis cells containing either a disruption or an ectopic overexpression of the $h s l 7$ gene exhibited cell length effects. The $\Delta_{810-2832} h s l 7$ mutant strains exhibit an increase in cell length, independent of mating-type background, while the $h s l 7^{\text {Otef }}$ mutant strains 
displayed a decrease in cell length, only significant in the $a 2 b 2$ mating-type background. The results were similar yet opposite to those in $s m u l$ mutant strains. Deletion of $h s l 7$ in S. cerevisiae caused a slight increase in cell size and an increase in bud length, while deletion of $s k b 1$ in S. pombe created a decrease in cell length (Gilbreth, et al., 1996 and 1998; Shulewitz, et al., 1999). Conversely, overexpression of $s k b l$ caused an increase in cell length. Both Hs17 and Skb1 have been shown to play a role in cell cycle regulation in S. cerevisiae and S. pombe, respectively, although resulting in opposite effects in the $\mathrm{G}_{2}-\mathrm{M}$ transition (Gilbreth, et al., 1996 and 1998; Ma, et al., 1996; Shulewitz, et al., 1999). Growth rate analysis and cell wall staining indicated no differences between the $h s l 7$ mutant strains and the wild type strains. Additionally, the $\Delta_{810-2832} h s l 7$ mutant strains did display small protrusions from colonies grown on YEPS media. Analysis of the micro-colonies of the $\Delta_{810-2832} h s l 7$ mutant strains indicated a "directional growth" phenotype, where groups of cells grew parallel to one another, yet independent from other groups of parallel cells giving rise to small protrusions from the general outline of the colony. An increase in cell to cell adhesion may explain the "directional growth" phenotype. Flo1 1p, a cell surface flocculin involved in cell-cell adhesion, is required for haploid invasive growth as well as diploid pseudohyphal development in S. cerevisiae (Palecek, et al., 2000). Mutating hsl7 in S. cerevisiae enhances agar invasion as well as filamentous growth independent of flo11 expression. In U. maydis, Hsl7 could play a similar role repressing cell to cell adhesion that may be required for filament formation.

The Hsl7 homologues of S. cerevisiae and S. pombe have been shown to play roles in the filamentous response to low ammonium conditions and hyperosmotic conditions, respectively (Fujita, et al., 1999 and Bao, et al., 2001). While the $\Delta_{810-}$ 
${ }_{2832} h s l 7$ and $h s l 7^{\text {Otef }}$ mutant strains were not sensitive to hyperosmotic conditions, nor to cell wall stressing agents or acid $\mathrm{pH}$ conditions, they did display perturbations in the filamentous response to low ammonium. The $\Delta_{810-2832} h s l 7$ and $h s l 7^{\text {Otef }}$ mutant strains displayed an increase and a decrease in the ability to form filaments on SLAD, respectively. Additionally, the $\Delta_{810-2832} h s l 7$ mutant strains also exhibited the "directional growth" previously observed on rich media. In S. cerevisiae, Hsl7 plays a role in the filamentous response to low ammonium conditions, initially by acting as a negative regulator of Ste20 in the filamentous response pathway (Fujita, et al., 1999). However, additional studies point to $\mathrm{Hsl7}$ regulating the filamentous response to low ammonium conditions via regulating Swel and the $\mathrm{G}_{2}-\mathrm{M}$ transition (La Valle and Wittenberg, 2001). Deletion of hsl7 leads to accumulation and stabilization of Swe1, with a freeze in the entry into mitosis, promoting cell elongation. Overall, this indicates that $h s l 7$, in addition, and potentially independent to $s m u l$, plays a role in cell length and the filamentous response to low ammonium conditions.

\section{Disrupting $s m u 1$ in a $\Delta_{810-2832} h s l 7$ background does not rescue any $\Delta_{810-}$} ${ }_{2832} h s / 7$ associated phenotypes. Studies of the Ste 20 homologues from S. cerevisiae and S. pombe indicated that the respective Hsl7 homologues interact with the PAK kinases both in vitro and in vivo (Fujita, et al., 1999; Gilbreth, et al., 1996 and 1998; Bao, et al., 2001). Yeast two-hybrid analysis indicated that the protein product of the predicted Hsl7 ORF of $U$. maydis interacted with Smu1 in vitro. Based on these results, a disruption of $s m u l$ was introduced in a $\Delta_{810-2832} h s l 7$ background to examine a possible genetic interaction between the two gene products. In both the S. cerevisiae and the S. pombe, the Hs17 homologue is upstream of the Ste20 homologue, modulating its function (Fujita, 
et al., 1999 and Bao, et al., 2001). The disruption of smul in the $\Delta_{810-2832} h s l 7$ background did not rescue any of the $\Delta_{810-2832} h s l 7$ associated phenotypes. Cell length increases, directional growth, and increases in the filamentous response to low ammonium conditions were unaffected in the double disruption strains compared with the $\Delta_{810}$ 2832hsl7 mutant strains. In addition, no new phenotypes were identified in the double disruption strains. These results stand at odds with models in both $S$. cerevisiae and $S$. pombe predicting the interaction of the Hsl7 homologues and PAK kinase (Ste20 and Shk1) homologues (Fujita, et al., 1999; Gilbreth, et al., 1996 and 1998; Bao, et al., 2001). Both Ste20 and Shk 1 have been shown to be downstream of the Hs17 homologue, whose function modulates the subsequent PAK kinase functions.

\section{Overexpressing smul in a $\Delta_{810-2832} h s l 7$ background exacerbates $\Delta_{810-2832} h s l 7$} associated phenotypes. To further explore a potential genetic interaction, an ectopic copy of $s m u l$ was overexpressed from the $\mathrm{P}_{\text {Otef }}$ promoter in the $\Delta_{810-2832} h s l 7$ background. Such double mutant cells were extremely elongated, above and beyond any previously examined strain in this work. Cell separation defects were observed only in the $a 2 b 2$ mating-type background. In addition, a filamentous colony morphology with septated hyphal cells was also observed. Overall this double mutant background exacerbates the phenotypes observed in the $\Delta_{810-2832} h s l 7$ background. Under conditions of low ammonium, the $\Delta_{810-2832} h s l 7 \mathrm{smul}^{\text {Otef }}$ mutant strain colonies displayed a hyperfilamentous response with extremely elongated filaments with massive chitin delocalization. And again mating-type specific phenotypes were observed, with the $a 2 b 2$ mating-type background exhibiting cytoplasmic evacuation. To better explore the observed cell wall defects in the $\Delta_{810-2832} \mathrm{hsl} 7 \mathrm{smul}{ }^{\text {Otef }}$ mutant strains, the cells were 
grown in liquid SLAD (low ammonium) media. Interestingly, only the $\Delta_{810-2832} h s l 7$ smu1 $1^{\text {Otef }}$ mutant strains were able to form pseudohyphal filaments when grown in liquid SLAD, whereas all other strains examined did not produce filaments. Unlike solid media, where growing cells quickly exhaust the local supply of ammonium, cells grown in liquid media have constant availability to nutrients, even those in low amounts, due to the continuous circulation that occurs in the liquid medium. Another requirement for filament formation may be contact with a solid surface as in solid media. Additionally, the filamentous response to SLAD conditions in solid media may be driven by a form of quorum sensing, where cell density plays a role in triggering the filamentous response. Quorum sensing has been observed in C. albicans (Hogan, 2006). Also, A. niger exhibited spatial regulation of genes involved in nitrate metabolism, independent of carbon source and nitrate concentration (Levin et al., 2007). Staining of the $\Delta_{810-2832} h s l 7$ ${ }_{s m u 1^{\text {Otef }}}$ mutant strains again revealed chitin delocalization while $\beta$-glucan localization was unaffected. Chitin, which normally localizes to the growing apical tip of U. maydis, as well as to the two septa formed during cytokinesis, was distributed though out the cell walls of the mutant strains. The chitin localization was not ubiquitous though, as regions of the pseudohyphal filaments did display more chitin localization than areas of the cell wall. Staining of the filaments with DAPI indicated an interesting dichotomy between the mating-type specific mutant strains. The $a l b l$ mutant strain exhibited a single long filament containing a single, centrally located, nucleus, whereas the $a 2 b 2$ exhibited true pseudohyphae that grew in a bi-polar fashion with each compartment containing a single nucleus. Taken as a whole, it appears that Smul, in concert with Hsl7, is involved in localizing chitin to the proper mother daughter septa in response to low ammonium 
conditions, and the response to this concerted action is mating-type dependent. In $U$. maydis, the activity of Hsl7 does not appear to regulate the activity of Smul, but instead the genetic manipulations introduced in the $\Delta_{810-2832} h s l 7 s m u l^{\text {Otef }}$ mutant strains promotes a synergism creating the hyper-filamentous response to low ammonium conditions.

The disruption of $\boldsymbol{h s} l \mathbf{7}$ does not alleviate the reduction in virulence associated with disruption of smu1. Smith et al. (2004) demonstrated that disruption of smu1 decreased virulence of $U$. maydis in a dosage dependent manner. This decrease was significant when both haploid strains were disrupted for smul. Kruskal-Wallis ANOVA analysis of the previously described mutant strains indicated no significant effects on virulence from $\Delta_{810-2832} h s l 7, h s l 7^{\text {Otef }}$, or $s m u l^{\text {otef }}$ mutant strains. The significant decrease in virulence of $\Delta s m u l$ mutant $(p<.001)$ was consistent with previous observations (Smith, et al., 2004), the $\Delta_{810-2832} h s l 7 \Delta s m u l$ mutant strain was significantly decreased for virulence compared to the wild type strains as well $(p<.001)$, yet no significant difference was observed between the $\Delta_{810-2832} h s l 7 \Delta s m u l$ and $\Delta s m u l$ mutant strains. However, the $\Delta_{810-2832} h s l 7 s m u I^{\text {Otef }}$ mutant strains exhibited a dramatic decrease in virulence, lower than all other strains examined. The decrease in virulence was significant in comparison to all previously examined strains, indicating that the role of Hs17, through Smu1, on pathogenicity is difficult to determine. The $\Delta_{810-2832} h s l 7 \mathrm{smul}$ Otef mutant strains did retain the ability to mate on YPD-charcoal media, however, the $a 2 b 2$ mating-type specific mutant strain exhibited the white aerial hyphae "fuz" of a positive mating reaction in the absence of the opposite mating partner. The $a 1 b 1 \Delta_{810-2832} h s l 7$ smul ${ }^{\text {Otef }}$ mutant strain, when mixed with the opposite mating partner, did display an increase in the mating response compared to wild type strains (Supplemental Fig. 5). It 
appears that the reduction in virulence in the $\Delta_{810-2832} 2 s l 7 \mathrm{smul}^{\text {Otef }}$ mutant strains is not due to inability to activate the $a$ locus controlling cell recognition and cell fusion, but could be an aspect of the $b$ locus that regulates sexual and pathogenic development.

Conclusions. I have demonstrated the cloning and characterization of the $\mathrm{Hsl} 7$ homologue from U. maydis and its genetic interaction with Smu1, the Ste20 homologue. The homologue described in this report, Hs17, has greater amino acid similarity to Skb1 from S. pombe, but shares many roles identified in both Hsl7 and Skb1 from S. cerevisiae and $S$. pombe, respectively. In yeast, Ste 20 potentially regulates both the mating response and morphogenesis subsets of the MAPK pathway (Roberts and Fink, 1994; Leberer, et al., 1997). Hsl7 interacts with Ste20 in a negative manner regulating morphogenesis through the MAPK under low ammonium conditions, but is independent of the mating reaction responsibilities of the MAPK (Fujita et al., 1999). In addition, Ste20 may play an indirect role in cell cycle regulation through actin polarization and septin formation (Cvrčková, et al., 1995; Holly and Blumer, 1999; Höfken and Schiebel, 2002). In contrast, in fission yeast, Shk1 acts parallel to the MAPK pathway in morphological responses but is predicted to act through the MAPK module in regard to the mating response (Marcus, et al., 1995). Here again, Skb1 acts upon Shk1, in a positive manner regulating cell cycle and the response to hyperosmotic conditions (Gilbreth, et al., 1996 and 1998; Bao, et al., 2001).

I demonstrated in $U$. maydis that Hsl7 negatively regulates cell length and the filamentous response to low ammonium conditions, partially though Smu1. In particular, disrupting $h s l 7$, while concomitantly overexpressing $s m u l$, negatively affects mother daughter cell separation in rich media, but only in the $a 2 b 2$ mating-type background. 
However, in low ammonium conditions both mating-type mutant strains are affected for cell separation. The $a l b 1$ mutant strain produces extremely long filaments with very few septa and a single nucleus, whereas the $a 2 b 2$ mutant strain produces pseudohyphae, each containing a single nucleus separated by mother daughter cell septa or cross wall septa. The defects in cell separation of the $\Delta_{810-2832} \mathrm{hs} / 7 \mathrm{smul}{ }^{\text {Otef }}$ mutant strains were similar to phenotypes observed in $U$. maydis $\Delta c d c 42, \Delta d o n 1$, and $\Delta d o n 3$ mutant strains. The $\Delta c d c 42, \Delta d o n 1$, and $\Delta d o n 3$ mutant strains, which do not display any aberrant cell morphology phenotypes, are defective in mother daughter cell separation, specifically, failing to create the second septum in the daughter cell between which vesicular elements and membrane aggregates accumulate (Weinzierl, et al., 2002 and Mahlert, et al., 2006). At closer examination, however, the phenotype associated with the $\Delta_{810-2832} h s l 7 \mathrm{smul}$ otef mutant strains are different than the daughter septum formation defects observed in $\Delta c d c 42, \Delta d o n 1$, and $\Delta d o n 3$ mutant strains. The $\Delta_{810-2832} h s l 7 s m u 1^{\text {Otef }}$ mutant strains are able to form both mother and daughter septa as well as cross wall septa and lateral buds. Also, the loss of the $c d c 42, d o n 1$, and don 3 gene products contribute to a polar growth pattern observed in the "don" mutant phenotype of U. maydis. In wild type haploid cells, budding alternates between the poles of cells rather than unipolar budding that is observed in pseudohyphae (Jacobs, et al., 1994). The failure of the cells to form the daughter septum and separate, in combination with the polar growth pattern, contributes to the "don" phenotype, where cells along the outer edge of the colony grow faster than the inner cells pulling them outward away from the center of the colony (Weinzierl, et al., 2002). The phenotypes exhibited by the $\Delta_{810-2832} h s l 7 \mathrm{smu1}{ }^{\text {Otef }}$ mutant strains also vary from the "don" mutant strains in that the center of the colony has not pulled away 
creating a "donut" phenotype with a hollow center. It is possible that the link between mother daughter cells is not strong enough in this specific mutant background to maintain the "don" phenotype. As the newer generations of cells grow outward, the fragmentation zones between the mother and daughter septa are pulled apart preventing the older, more centrally located cells from being torn from the center of the colony. It is also possible that the $\Delta_{810-2832} h s / 7 \mathrm{smul}^{\text {Otef }}$ mutant strains do not undergo the unipolar growth pattern observed in the $\Delta d o n 1, \Delta d o n 3$, and $\Delta c d c 42$ mutants, but rather the cells undergo a bipolar growth pattern.

Recent work has delineated Cdc42 and Don3 as independent co-stimulators of cytokinesis with Don 3 activity preceding Cdc 42 activity in formation of the secondary septin (Böhmer, et al., 2008). Here Don3 is predicted to phoshporylate, subsequently activating, a putative landmark protein allowing for $\mathrm{Cdc} 42$ dependent septin and actomyosin ring formation. Rho-type GTPases have been shown to be involved in cytoskeletal organization and formation of the actomyosin ring in yeast (Tapon and Hall, 1997; Eby, et al., 1998; Nelson, 2003). Additionally Ste20 is also involved in actin cytoskeleton organization and is a downstream effector Cdc42 in this process (Eby, et al., 1998). It is possible that Smu1 may be a downstream effector of Cdc42 in U. maydis, potentially regulating actin cytoskeleton dynamics and formation of the actomyosin and septin rings. An interaction through yeast two-hybrid analysis indicated that $\mathrm{Cdc} 42$ interacts with the CRIB domain of Smul in vitro (Leveleki, et al., 2004). This, coupled with the positive yeast two-hybrid interaction between Smu1 and Hs17, could explain the inability of $\Delta_{810-2832} h s l 7 \mathrm{smu} 1^{\text {Otef }}$ mutant strains to undergo mother daughter cell separation under low ammonium conditions, ultimately enhancing the filamentous 
response. Here Hsl7 would be acting as a negative regulator of Smu1. However, this idea fails to explain the inability of $s m u l$ disruption mutants to rescue phenotypes associated with loss of $h s l 7$. An alternate hypothesis, i.e., loss of $h s l 7$ potentially delaying cell cycle progression, while overexpression of $s m u l$ promotes the filamentous response, possibly via the MAPK pathway, explains the observed phenotypes in both the $\Delta_{810-2832} h s l 7 \Delta s m u l$ mutant strains and the $\Delta_{810-2832} h s l 7$ smul ${ }^{\text {Otef }}$ mutant strains. Hsl7 may act on cell cycle, promoting entry into mitosis by targeting Weel for degradation.

Absence of Hsl7 would delay the cell cycle leading to an increase in cell length as is observed in S. cerevisiae (Shulewitz, et al., 1999). This cell cycle delay in the absence of Hs17 may act in concert with the overexpression of smul, leading to up regulation of the filamentous response to low ammonium conditions that is exacerbated under conditions that promote this response. 


\section{CHAPTER III}

\section{CLA4, BUT NOT RAC1, REGULATES THE FILAMENTOUS RESPONSE TO LOW AMMONIUM CONDITIONS}

\section{INTRODUCTION}

Many fungal pathogens exhibit a dimorphism in which the cells transition from either a budding morphology to a hyphal morphology or vice versa. The morphological transition of dimorphic fungi is governed by a several different regulatory pathways, which mediate cell cycle regulation, cytoskeletal organization, and differential gene expression. Members of the Rho/Rac family of GTP-binding proteins (GTPases), including $\mathrm{Cdc} 42$ and Rac1, have been implicated in many of these pathways, regulating actin organization, cell polarity, and cytokinesis (Nakano, et al., 1997; Cabib, et al., 1998; Madden and Snyder, 1998; Lengeler, et al., 2000; Tolliday, et al., 2002; Nelson, 2003; Etienne-Manneville, 2004; Bassilana and Arkowitz, 2006; Mahlert et al., 2006). The activity of these GTP-binding proteins is dependent upon two additional groups of proteins, guanine exchange factors (GEFs), which activate their respective targets, and GTPase activating proteins (GAPs) that inactivate the GTPases. When activated, Rho/Rac GTPases modulate localization and activity of several downstream effectors including p21-activated protein kinases (PAKs). The PAKs comprise a large family of serine/threonine protein kinases that regulate many cell processes including cell cycle, cytoskeletal organization, and polar growth (Cvrčková, et al., 1995; Marcus, et al., 1995; 
Gilbreth, et al., 1996 and 1998; Eby, et al., 1998; Holly and Blumer, 1999; Bao, et al., 2001; Höfken and Schiebel, 2002; Qyang, et al., 2002; Wiley et al., 2003; Leveleki, et al., 2004; Smith, et al., 2004). Evidence suggests that PAKs regulate the conserved mitogen activated protein kinase (MAPK) pathway in several of these processes (Lengeler et al., 2000; García-Pedrajas, et al., 2008). The MAPK pathway is a threecomponent module consisting of three protein kinases, a MAPK kinase kinase (MAPKKK), a MAPK kinase (MAPKK), and a MAP kinase (MAPK), which regulate the dimorphic transition in response to mating factors, environmental conditions, or for virulence (Lengeler et al., 2000; García-Pedrajas, et al., 2008). The pathways in Saccharomyces cerevisiae regulating mating, growth in hyperosmotic conditions, and pseudohyphal development are some of the best understood (Lengeler et al., 2000; García-Pedrajas, et al., 2008).

In the basidiomycete fungus Ustilago maydis, unicellular haploid cells reproduce by budding. Haploid cells of opposite mating backgrounds can fuse and form a diploid filamentous dikaryon. This dikaryon can subsequently infect maize (Zea mays). Cell fusion and pathogenic development are controlled by two separate loci, $a$ and $b$. The $a$ locus encodes a pheromone and pheromone receptor involved in cell recognition and cell fusion. Subsequent pathogenic development is dependent upon the $b$ locus which encodes two homeodomain proteins, bE and bW (Kronstad and Leong, 1989). After cell fusion, dimerization of the $\mathrm{bE}$ and $\mathrm{bW}$ proteins from opposite mating-type backgrounds, yields a functional heterodimer that regulates sexual development and pathogenicity (Kämper, et al., 1995). Differentiation into the infectious dikaryon is dependent upon the activity of this $b$ heterodimer. However, U. maydis cells can also undergo similar 
filamentous differentiation in response to environmental conditions. Lipids, acidic $\mathrm{pH}$, and low ammonium conditions can trigger the filamentous response in a b locusindependent manner (Ruiz-Herrera, et al., 1995; Smith et al., 2003; Klose, et al., 2004).

The highly conserved GTPase, Cdc42 of S. cerevisiae regulates both bud formation and polarized cell growth, though two well studied PAKs, Ste20 and Cla4. Through the Cdc42 specific GEF, Cdc24, Cdc42 localizes to the bud neck and contributes, in a Cla4 dependent manner, to the septin ring formation (Versele and Thorner, 2004). Septin ring formation is a critical step in the cell cycle. The failure of septin ring formation results in a Swe1-dependent $\mathrm{G}_{2}$ delay in cell cycle. Cdc42 and Ste20 also contribute to polarizing the actin cytoskeleton and polarized cell growth. This occurs partially though the MAPK pathway in response to mating cues, as well as low ammonium conditions (Eby, et al., 1998; Lengeler, et al., 2000; Lamson, et al., 2002). While $S$. cerevisiae contains only Cdc42, U. maydis contains $\mathrm{Cdc} 42$ and a second Rho/Rac GTPase, Rac1. In U. maydis, Cdc42, through its specific GEF, Don1, regulates septum formation and mother daughter cell separation (Weinzierl, et al., 2002; Mahlert, et al., 2006; Böhmer, et al., 2008). In U. maydis, the activity of Rac1, regulated by the Rac1 specific GEF, Cdc24, focuses on bud formation and morphology, as well as polar growth. Rac1, via Cdc24, localizes to the polar tip of the bud and directs localized cell wall deposition (Leveleki, et al., 2004; Mahlert, et al., 2006). The deposition of cell wall material is Cla4 dependent and promotes apical growth of the bud. Deletion mutants of both $\mathrm{racl}$ and cla 4 are unable to form bud necks or undergo proper bud formation; in addition, neither mutant has the ability to form $b$ locus-dependent filaments. Thus Rac1 may act as the master regulator of the filamentous response through Cla4. 
To date, no work has focused on the role Racl or Cla4 may have in the filamentous response to environmental conditions, such as low ammonium conditions. In contrast the Ste 20 homologue, Smu1, is known to have a role in the filamentous response to low ammonium conditions (Smith, et al., 2004; Chapter II). Smu1 appears to regulate the filament formation under low ammonium conditions in a positive fashion. In this study, I explore the roles Rac1 and Cla4 in the filamentous response to low ammonium conditions. I demonstrate that deletion of $\mathrm{racl}$ leads to a severe delay in filament formation and a dramatic reduction in filament number compared to wild type strains. Conversely, deletion of cla4 leads to a hyper-filamentous response under low ammonium condition compared to wild type strains.

\section{MATERIALS and METHODS}

Strains. Ustilago maydis strains used in this study that were not previously listed in Chapter 2 are listed in Table 9.

TABLE 9. $U$. maydis strains and plasmids used in this study.

\begin{tabular}{|c|c|c|}
\hline Strain / Plasmid & Genotype & Reference \\
\hline \multicolumn{3}{|l|}{ Fungal Strains ${ }^{A}$} \\
\hline FB1 WT & $a l b 1$ & Banuett, et al, 1989 \\
\hline$\Delta c l a 4$ al & alb1 cla4::hyg ${ }^{R}$ & Leveleki et al., 2004 \\
\hline cla $^{\text {Otef }}$ al & $a l b 1 P_{\text {ote }} c l a 4, c b x^{R}$ & This Study \\
\hline $\mathrm{racl}^{\text {Otef }}$ al & $a l b l P_{\text {otef }} r a c l, c b x^{R}$ & Pham, et al., 2009 \\
\hline FB2 WT & $a 2 b 2$ & Banuett, et al, 1989 \\
\hline$\Delta c l a 4 a 2$ & $a 2 b 2$ cla4::hyg ${ }^{R}$ & Leveleki et al., 2004 \\
\hline $\mathrm{cla}^{\text {Otef }} a 2$ & $a 2 b 2 P_{o t e f}$ cla4, $c b x^{R}$ & This Study \\
\hline$\Delta r a c l a 2$ & $a 2 b 4$ racl::nour $r^{R}$ & Leveleki et al., 2004 \\
\hline \multicolumn{3}{|l|}{ Plasmid } \\
\hline pOtef-cla4 & $P_{\text {otef }}$ cla4,$c b x^{R}$ & This Study \\
\hline
\end{tabular}

${ }^{\mathrm{A}}$ All mutant strains were made in the wild type background strains listed. 
Primer Design. Primers were designed as previously described (Materials and methods, Ch. 2) and are listed in Table 10.

TABLE 10. Primers used for PCR and Sequencing.

\begin{tabular}{ll} 
Primers & Sequences $\left(5^{\prime} \rightarrow 3^{\prime}\right)$ \\
\hline NCla5 & TTCTAGACGCCACCTCTGGACTACAA \\
Cla43K & CGGTACCTGAAAAGGGAAGGGAGT \\
\hline
\end{tabular}

Genetic Manipulation and Vector Construction. Overexpression of cla4 in $U$. maydis was obtained by homologous recombination as described previously (Brachmann et al., 2004). The cla 4 expression construct was produced by amplifying the cla 4 ORF with NCla5 and Cla43K primers and cloning the product into pCR2.1 TOPO (Invitrogen). To provide constitutive expression, the cla4 ORF was then excised and cloned after the $P_{\text {ote }}$ promoter into the BamHI and NotI sites of the p123 vector (Weber, et al., 2006). The $c l a 4^{\text {Otef }}$ expression vector was linearized using $S s p I$ before transforming U. maydis, under selection for carboxin resistance (Brachmann et al., 2001). Recombination at the ip locus would yield the carboxin resistant transformants. The $c l a 4^{\text {Otef }}$ expression vector was digested with restriction endonucleases, purified, and used to transform $U$. maydis protoplasts. Potential transformants were verified by PCR.

PCR, growth conditions, cell length, statistical analysis, staining, micro-colonies, and microscopy have been described previously (Materials and methods, Chapter II).

\section{RESULTS}

Acla4 mutant strains are affected in cell length, chitin localization, and cell separation. Previously Cla4 was found to be important for proper budding and cell morphology (Leveleki, et al., 2004). Deletion of the cla4 ORF afffected cell morphology, causing the cells to appear fatter than the wild type strains (Fig. 25). In 


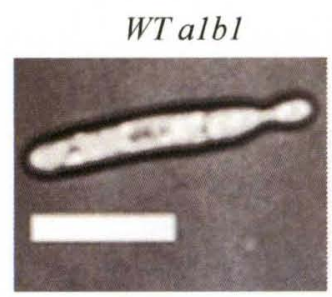

WT $a 2 b 2$
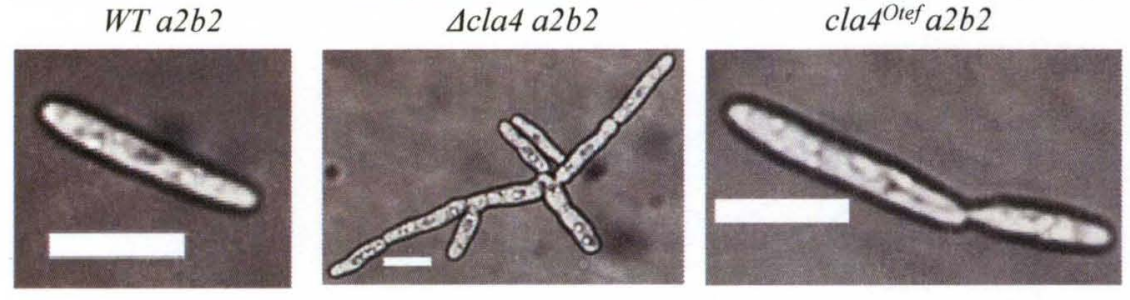

Figure 25: Cla4 plays a role in cytokinesis and cell length. $\Delta$ cla 4 cells display an increase in cell length in the $a 2 b 2$ mating-type strain only, while cla $4^{\text {Otef }}$ cells exhibit an increase in cell length only in the alb1 mating-type specific strain. a2b2 $\Delta c l a 4$ cells also exhibited cytokinesis defects. Scale bars, $10 \mu \mathrm{m}$.

addition, cytokinesis was affected in the $\Delta c l a 4$ mutant strains, with the greater defect occurring in the a2b2 mating-type background (Fig. 25 and Leveleki, et al., 2004). The cla 4 mutant strains were affected in cell length, primarily in the $a 2 b 2$ mating-type background, but this phenotype was not explored (Leveleki, et al., 2004). In the current study, examination of the $\Delta$ cla 4 mutant strains identified a significant increase in cell length that was specific to the $a 2 b 2$ mating-type background. No significant increase in cell length was observed in the albl mating-type background (Table 11). Comparisons between the $\Delta c l a 4 a 1 b 1$ and $a 2 b 2$ mutant strains demonstrated a significant difference between the mating-type backgrounds (Table 12). This mating- type specific increase in cell length stands in sharp contrast to the decrease in cell lengths observed in $\Delta s m u 1$ mutant strains. The significant changes in cell length in both the $\Delta c l a 4$ and $\Delta s m u 1$ mutant strains compared to wild type strains are confined to the $a 2 b 2$ mating-type background (Table 12). Comparisons of the mating-type specific effects on cell length between the $\Delta c l a 4$ and $\Delta s m u 1$ mutant strains indicated that the lengths were significantly 
TABLE 11. Measures of cell length across all strains.

\begin{tabular}{|c|c|c|c|c|}
\hline Strain & $\mathrm{n}$ & Length $^{\mathrm{A}}$ (in $\mu \mathrm{m}$ ) & Comparison & $p^{B}$ \\
\hline WT albl & 91 & $19.27+/-.43$ & $W T a l b 1$ v. WT a2b2 & N.S. \\
\hline$\triangle c l a 4$ al & 408 & $19.48+/-.30$ & WTalbl v. $\Delta c l a 4$ al & N.S. \\
\hline Asmul al & 380 & $18.64+/-.16$ & WT albl v. $\Delta s m u l$ al & N.S. \\
\hline $\operatorname{cla}^{\text {Otef }}$ al & 367 & $21.22+/-.25$ & WTalbl v. cla $4^{\text {Otef }}$ al & $>.05$ \\
\hline smul $I^{\text {otef }}$ al & 308 & $21.75+/-.28$ & $W T a l b l$ v. smul Otef $a l$ & $>.001$ \\
\hline$W T a 2 b 2$ & 213 & $19.14+/-.31$ & & \\
\hline$\Delta c l a 4$ a 2 & 111 & $24.05+/-.83$ & $W T a 2 b 2$ v. $\triangle c l a 4 a 2$ & $>.001$ \\
\hline$\Delta s m u l$ a2 & 281 & $17.64+/-.18$ & $W T a 2 b 2$ v. $\Delta s m u 1 a 2$ & $>.05$ \\
\hline $\operatorname{cla}^{\text {Olef }}$ a2 & 505 & $19.69+/-.20$ & $W T a 2 b 2$ v. cla $4^{\text {tef }} a 2$ & N.S. \\
\hline smul ${ }^{\text {otef }} a 2$ & 224 & $19.66+/-.30$ & $W T a 2 b 2$ v. smu $1^{\text {Otef }} a 2$ & N.S. \\
\hline
\end{tabular}

${ }^{A}$ Cell length values are averages, +/- S.E.

B Statistical analysis was performed using a one way ANOVA with

a Dunnett's Multiple Comparison Test.

N.S. $=$ Not Significant

TABLE 12. Mating-type specific cell length differences.

\begin{tabular}{|c|c|c|c|c|}
\hline Strain & $\mathrm{n}$ & Length $^{\mathrm{A}}$ (in $\mu \mathrm{m}$ ) & Comparison & $p^{B}$ \\
\hline$\triangle c l a 4$ al & 408 & $19.48+/-.58$ & & \\
\hline Asmul al & 380 & $18.64+/-.31$ & $\Delta c l a 4$ al vs. $\Delta$ cla4 a2 & $>.001$ \\
\hline cla $^{\text {Otef }}$ al & 367 & $21.22+/-.25$ & Aclat al vs. Asmul al & $>.001$ \\
\hline smul ${ }^{\text {otef }}$ al & 308 & $21.75+/-.48$ & ${ }_{\text {cla }}{ }^{\text {Otef }}$ al vs. smul $I^{\text {otef }}$ al & N.S. \\
\hline$\Delta c l a 4$ a2 & 111 & $24.05+/-.85$ & & \\
\hline$\Delta s m u 1$ a2 & 281 & $17.64+/-.29$ & cla $^{\text {Otef }}$ al vs. clat $4^{\text {Otef }}$ a2 & $>.001$ \\
\hline $\mathrm{cla}^{\text {Otef }}$ a2 & 505 & $19.69+/-.20$ & $\Delta c l a 4$ a2 vs. $\Delta$ smul a2 & $>.001$ \\
\hline$s m u I^{\text {olef }} a 2$ & 224 & $19.66+/-.44$ & cla $^{\text {Otef }}$ a2 vs. smul ${ }^{\text {Otef }}$ a 2 & N.S. \\
\hline
\end{tabular}

${ }^{A}$ Cell length values are averages, +/- S.E.

B Statistical analysis was performed using a Student's t-test.

N.S. $=$ Not Significant

different, independent of mating-type background. 
Leveleki, et al. also indicated cell wall defects in chitin localization but not in $\beta$ glucan localization. Staining of the $\Delta c l a 4$ mutant strains with WGA and CFW, confirmed this previous work (Fig. 26, see arrows). Cross walls stained readily with

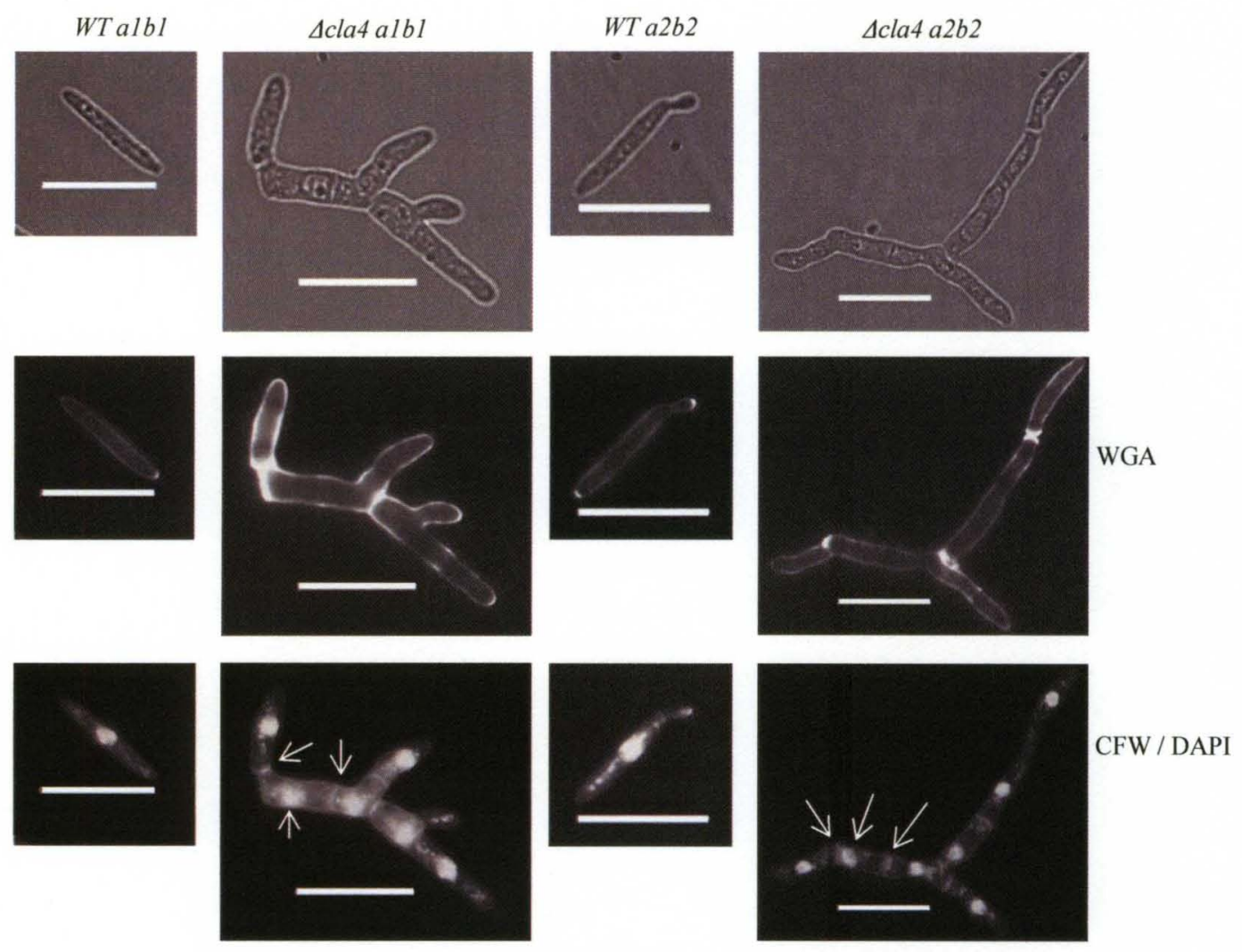

Figure 26: $\Delta c l a 4$ strains are multinucleic and exhibit chitin delocalization: $\Delta c l a 4$ cells appear fatter than wild type cells and when visualized with CFW, contain several cross walls (See arrows). The cells exhibit chitin delocalization when stained with WGA though. In addition, both matingtype strains contained several nuclei, separated by cross walls when stained with DAPI (See arrows). Scale bars, $10 \mu \mathrm{m}$.

CFW, but not with WGA indicating that chitin does not localize to the cross wall septa, whereas the $\beta$-glucans do localize to the septa. In addition, chitin deposition occurs all along the cell wall in the $\Delta c l a 4$ mutant strains, not to the polar tip as in the wild type strains (Fig. 26). Also, co-stained with CFW, DAPI indicated that each compartment contained a single nucleus, further supporting that the $\Delta c l a 4$ mutant strains divide by contraction at a central septum (Fig. 26, see arrows, and Leveleki, et al., 2004). 
Overexpression of cla 4 was generated by amplifying the cla 4 ORF and cloning it after the $\mathrm{P}_{\text {otef }}$ promoter. The linearized plasmid was then integrated into the $i p$ locus of the FB1 $a 1 b 1$ and FB2 a $2 b 2$ strains of $U$. maydis and confirmed by PCR (data not shown). Examination of the $\mathrm{cla}^{\text {Otef }}$ mutant strains did not display any aberrant cell morphologies other than cell elongation (Fig. 26). Also the $c l a 4^{\text {Otef }}$ mutant strains did not display any cell wall defects when visualized with CFW and WGA (Fig. 27). Cell length
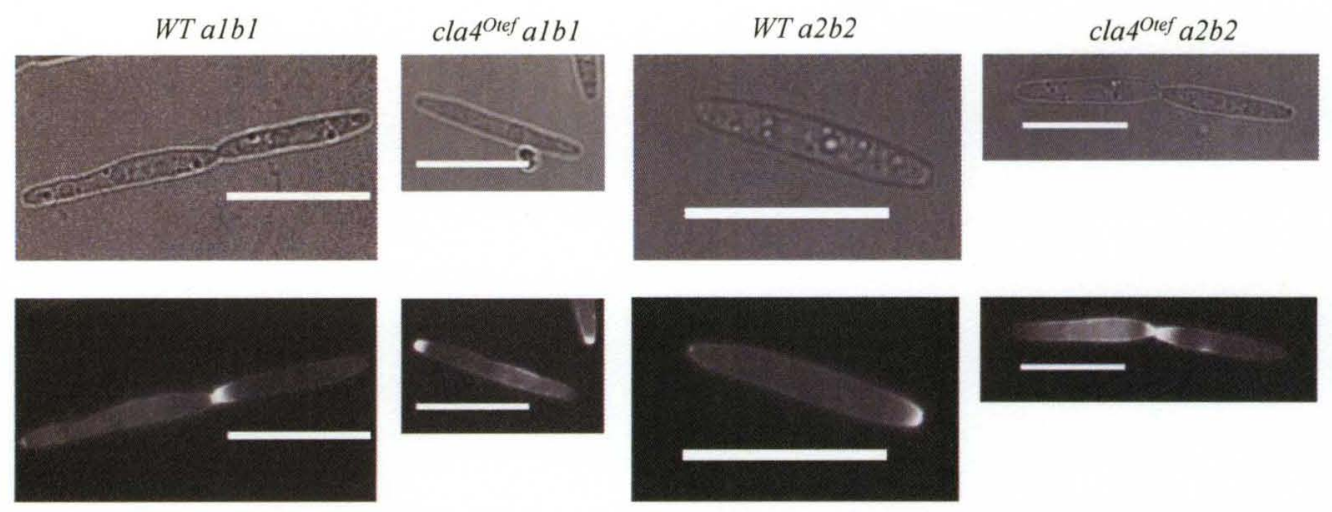

WGA
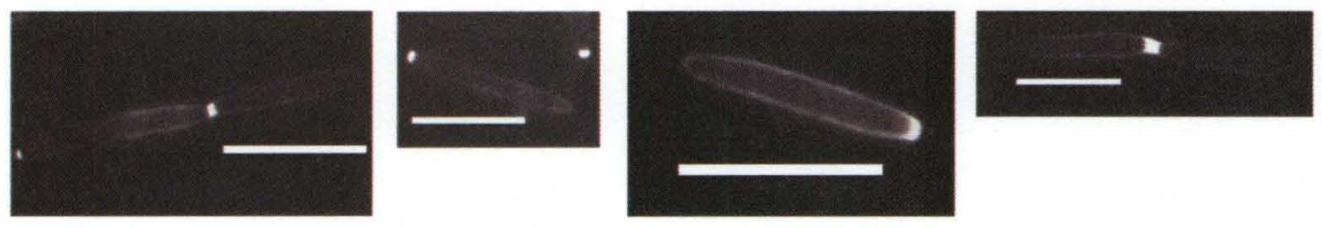

CFW

Figure 27: $\mathrm{cla}^{\text {Otef }}$ strains did not exhibit any defects in cell wall localization when stained with CFW and WGA. When grown in rich media, $\mathrm{cla}^{\mathrm{Otef}}$ strains did not display any defects in cell wall localization when stained with CFW and WGA. Scale bars, $10 \mu \mathrm{m}$.

measures indicated a significant increase in length, but only in the $a 1 b 1$ mating-type background, while the a2b2 mating-type background did not display any increase in cell length (Table 11). Comparison between the albl cla ${ }^{\text {Otef }}$ and $a 2 b 2 \mathrm{cla}^{\text {Otef }}$ mutant strains indicated a significant difference in cell length between the mating-type backgrounds (Table 12). This result is identical to the cell length phenotype observed in the smul ${ }^{\text {Otef }}$ mutant strains, where the $a l b l$ mating-type background displayed an increase in cell length, while the $a 2 b 2$ did not (Table 11). In addition, no significant difference was observed when the $\mathrm{cla} 4^{\text {Otef }}$ and $\mathrm{smul} \mathrm{O}^{\text {Otef }}$ mutant strains of the same mating-type 
background were compared (Table 12). While these results are in concordance with the mating-type specific phenotypes previously observed in both $\Delta c l a 4$ and $\Delta s m u l$ strains, the overall comparisons between the similarity of overexpressing either cla4 or smuland the differences of disrupting either cla4 or smul indicate a complex relationship between the two kinases.

\section{The colonies of the $\Delta c l a 4$ mutant strains are altered in the filamentous} response to low ammonium conditions. The $\Delta c l a 4$ mutant strains do not form filaments during the mating reaction and in planta (Leveleki et al., 2004). However, filamentation in response to low ammonium conditions in vitro was not examined. With the cell morphology phenotypes in mind, both colony and micro-colony morphologies of $\triangle c l a 4$ and $c l a 4^{O t e f}$ mutant strains were compared. When grown on YEPS media, neither the $\Delta c l a 4$, nor the $c l a 4^{\text {Otef }}$ mutant strains displayed any difference in colony morphology compared to the wild type progenitor strains (Fig. 28A). To explore colony morphology at the cell level, micro-colonies were utilized. On YEPS, at 20x and 60x magnification, the colony morphology of both the $\Delta c l a 4$ and $c l a 4^{\text {otef }}$ mutant strains did not vary from the wild type strains (Fig. 28B). The alb1 $\Delta c l a 4$ mutant strain did consistently exhibit filamentous cells that extended out away from the colony center (Fig. 28B), however, only a few filaments formed from each colony. It does appear that the $\Delta$ cla 4 mutant strains, particularly the $a 1 b 1$ mating-type strain, have the ability to form filaments on rich media.

To explore if the cla4 mutants have the ability to form filaments in response to environmental cues, the strains were grown in low ammonium conditions. The $\Delta c l a 4$ mutant strains did form filaments on low ammonium, and mating-type specific 


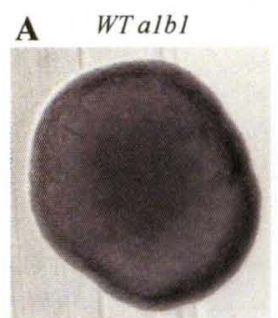

WT $a 2 b 2$

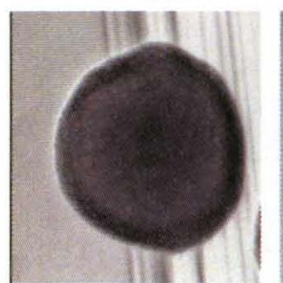

B

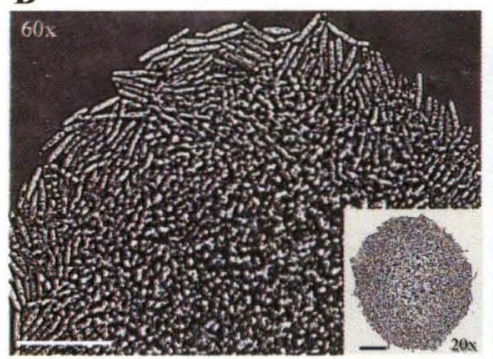

WT $a 2 b 2$

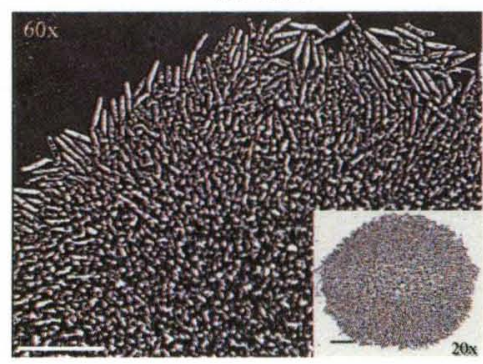

cla $4^{\text {Otef }} a \mathrm{lbl}$

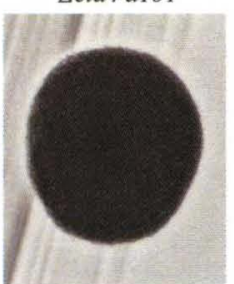

$\triangle c l a 4 a 2 b 2$

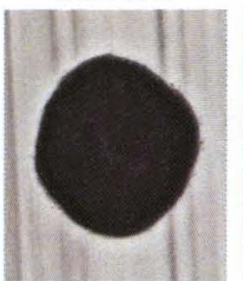

cla $4^{\text {Otef }} a 2 b 2$
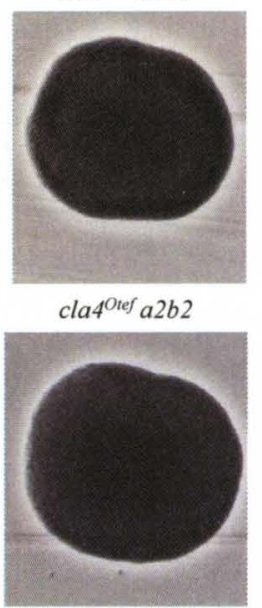

$\triangle$ cla4 albl

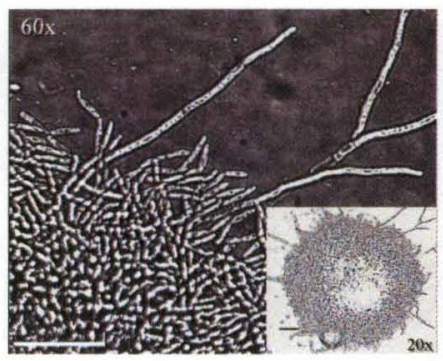

$\triangle c l a 4 a 2 b 2$

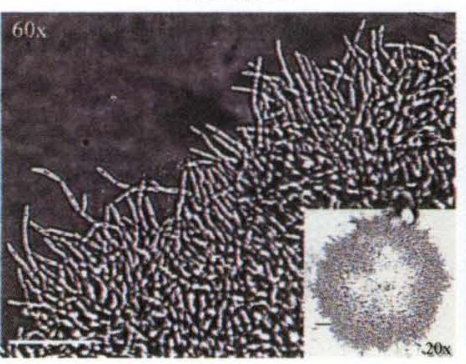

Figure 28: Cla4 mutant strains are unaffected for colony morphology on rich media. Neither $\Delta$ cla 4 nor cla $4^{\text {Olef }}$ colonies display any defects in colony morphology on rich media (A). The micro-colonies of both the $\Delta$ cla 4 and cla $4^{\text {Otef }}$ strains did not differ to that of the wild type strains, though a few cells of the albl $\Delta$ cla 4 strain did form filaments (B). Scale bars, $50 \mu \mathrm{m}-20 \mathrm{x}$ inset image, $40 \mu \mathrm{m}-60 \mathrm{x}$ image.

differences in response were observed (Fig. 29A). The alb1 $\Delta$ cla4 mutant strain

produced single, dramatically elongated and twisted filaments compared to the wild type albl strain (Fig. 29A). The filaments exhibited by the albl $\Delta$ cla 4 mutant strain appeared to have a similar darkened region at the end of each filament advancing from an empty filament, reminiscent of a phenotype observed in the $a 2 b 2 \Delta_{810-2832} h s l 7 \mathrm{smul} 1^{\text {otef }}$ mutant strain (Fig. 29A, see arrow; compare with Fig. 14A, Chapter II). The a2b2 $\Delta c l a 4$ mutant strain displayed a smaller colony size with a thicker filament size when compared 

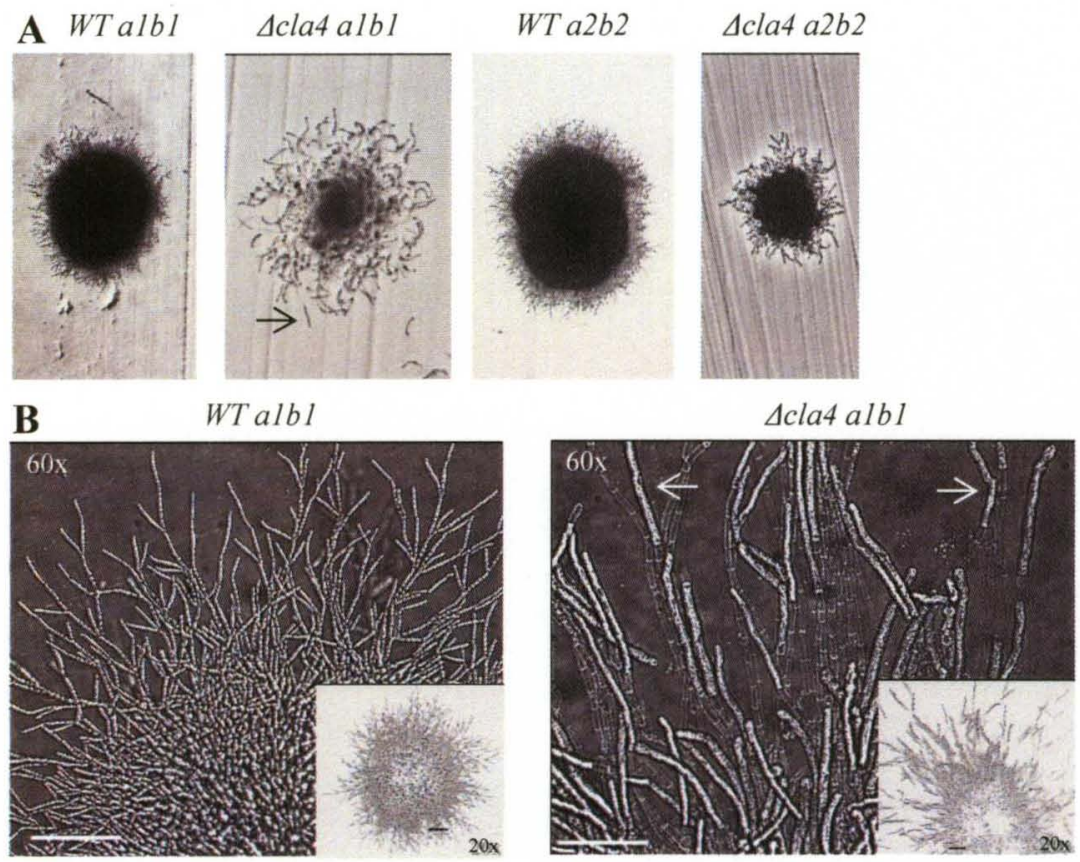

$W T a 2 b 2$

$\triangle c l a 4$ a 2 b2
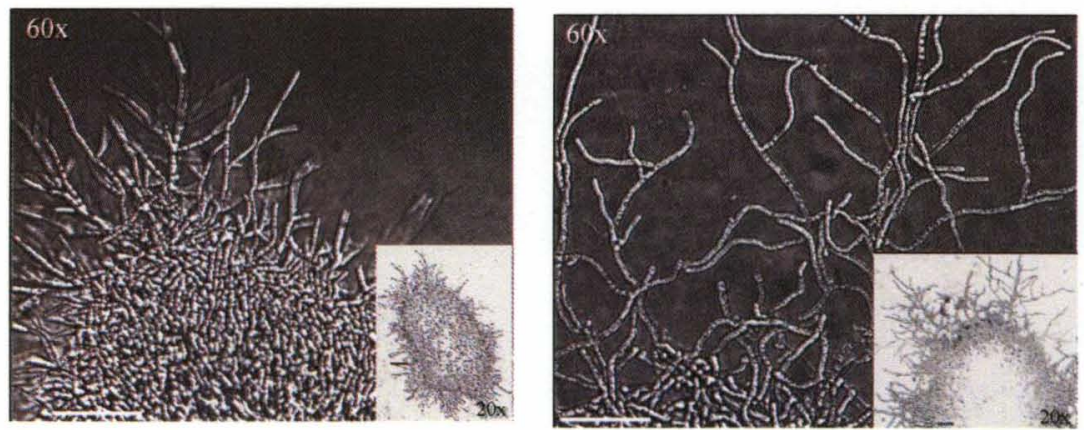

Figure 29: On SLAD media, the $\Delta$ cla4 strains display aberrant filament formation. The albl $\Delta$ cla 4 strain produces exaggerated filamentous colonies, while the a2b2 $\Delta$ cla 4 strain is reduced in the ability to filament on SLAD compared with the wild type strains. Also filaments of the albl $\triangle$ cla 4 strain exhibited darkened regions at the tips of empty filaments (A, see arrows). Micro-colonies of the alb1 $\Delta$ cla4 strain displayed extremely elongated, unbranched filaments with the cytoplasm compressed to the tip by retraction septa, while the $a 2 b 2 \Delta c l a 4$ strain exhibited elongated, highly branched filaments (B, see arrows). Scale bars, $50 \mu \mathrm{m}-20 \mathrm{x}$ inset image, $40 \mu \mathrm{m}$ with the filaments of the wild type $a 2 b 2$ strain (Fig. 29A). Micro- colonies were employed to then examine filament formation in more detail. At 20x magnification, the filaments formed by the albl $\Delta c l a 4$ mutant strain were much thicker and more elongated compared to the wild type strain, although they appeared to be single, unbranched filaments (Fig. 29B, inset). The a2b2 $\Delta$ cla 4 mutant strain, similar to the alb1 $\Delta c l a 4$ mutant strain also formed thick, elongated filaments. However, unlike the filaments 
produced in the $a l b 1$ mutant strain, the filaments produced in the $a 2 b 2$ mutant strain were branched (Fig. 29B, inset). At 60x magnification, the filaments seemingly had the cytoplasm confined to the polar tip by retraction septae. The filaments produced by the a2b2 Acla4 mutant strain were highly branched filaments, not displaying any of the septated hyphal attributes observed in the alb1 $\Delta$ cla4 mutant strain (Fig. 29B). Neither cytoplasmic evacuation nor septae were observed in the a2b2 $\Delta$ cla4 mutant strain.
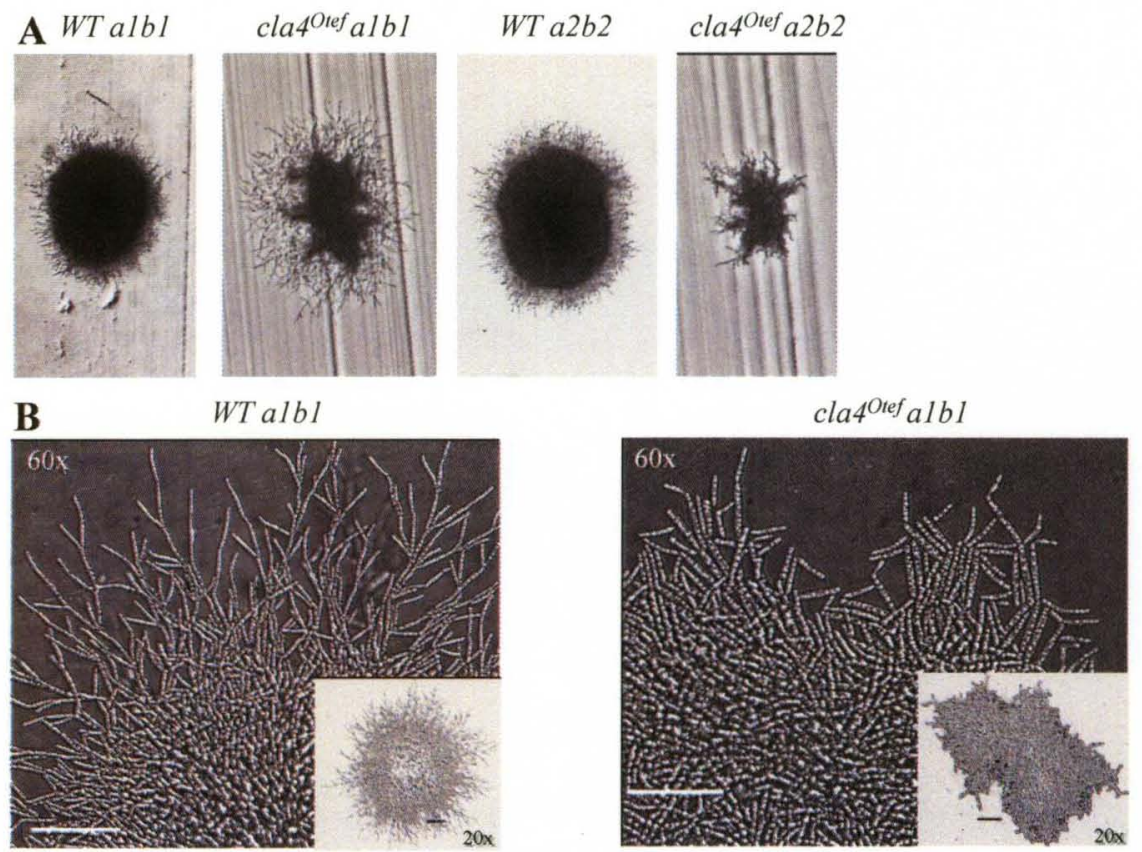

WT $a 2 b 2$
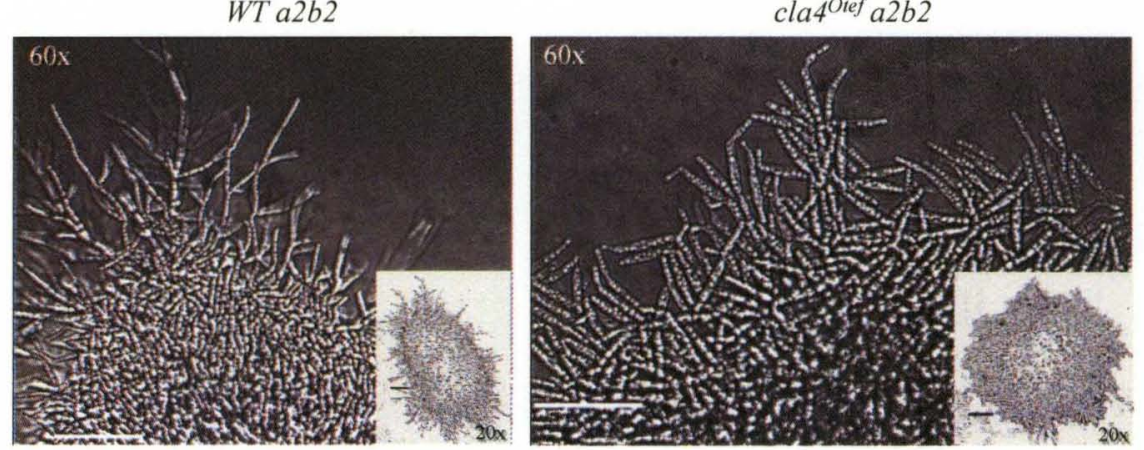

Figure 30: The $a 1 b 1$ cla $4^{\text {Otef }}$ mutant strain exhibits an increase in filamentation; however the $a 2 b 2$ cla $4^{\text {Otef }}$ mutant strain is reduced in the filamentous response on SLAD. cla $^{\text {Otef }}$ colonies exhibit an increase in filament formation in the $a 1 b 1$ mating-type background, while a decrease is observed in the a2b2 mating-type background (A). Micro-colonies of the $c l a 4^{\text {Otef }}$ strains exhibit a lack of filament formation at the cellular level, independent of mating-type background (B). Scale bars, $50 \mu \mathrm{m}-20 \mathrm{x}$ inset image, $40 \mu \mathrm{m}-60 \mathrm{x}$ image. 
The $c l a 4^{\text {Otef }}$ mutant strains formed filaments in response to SLAD media, with the albl cla $4^{\text {Otef }}$ mutant strain showing an increase in the production of filaments compared to the wild type $a 1 b 1$ strain (Fig. 30A). However, the colonies of the $a 2 b 2 c l a 4^{\text {Otef }}$ mutant strain were smaller and exhibited a decrease in filament formation in response to low ammonium conditions compared to their wild type progenitor strain (Fig. 30A). To further explore the mating-type differences observed in the colony morphology of the cla $^{\text {Otef }}$ mutant strains, micro-colonies were again employed. At $20 \mathrm{x}$ magnification, both the $a l b 1$ and the $a 2 b 2 c l a 4^{\text {Otef }}$ mutant strains displayed a dramatic reduction in the ability to form filaments on SLAD (Fig. 30B, inset). At 60x magnification, no filamentous cells appeared, in contrast to what was observed in the wild type strains (Fig. 30B). The reduction of the filamentous formation of the $a 1 b 1$ cla $4^{\text {otef }}$ mutant strain on SLAD microcolonies stands at odds with the colony morphology of albl cla $4^{\text {Otef }}$ under low ammonium conditions.

\section{Acla4 mutant strains are filamentous and display cell wall abnormalities in}

liquid SLAD. The $\Delta c l a 4$ mutant strains formed filaments when grown overnight in liquid SLAD, similar to the $\Delta_{810-2832} \mathrm{hsl} / \mathrm{smu1} \mathrm{I}^{\text {Otef }}$ mutant strains. The $\Delta c l a 4$ mutant strains were grown in liquid SLAD and stained with WGA and CFW. The albl $\Delta c l a 4$ mutant strain formed long, single, septated hyphae, whereas the a2b2 $\Delta c l a 4$ mutant strain exhibited large multi-branched hyphae with few cross wall septa (Fig. 31, see arrows). The albl $\Delta$ cla4 mutant strain displayed regularly spaced retraction septa when grown in liquid as was observed on the micro-colonies. Closer examination the $a 2 b 2 \Delta c l a 4$ mutant strain indicated that the branching of the hyphae occurred at cross wall septae. Both strains also displayed the same chitin localization defects observed when grown in rich 


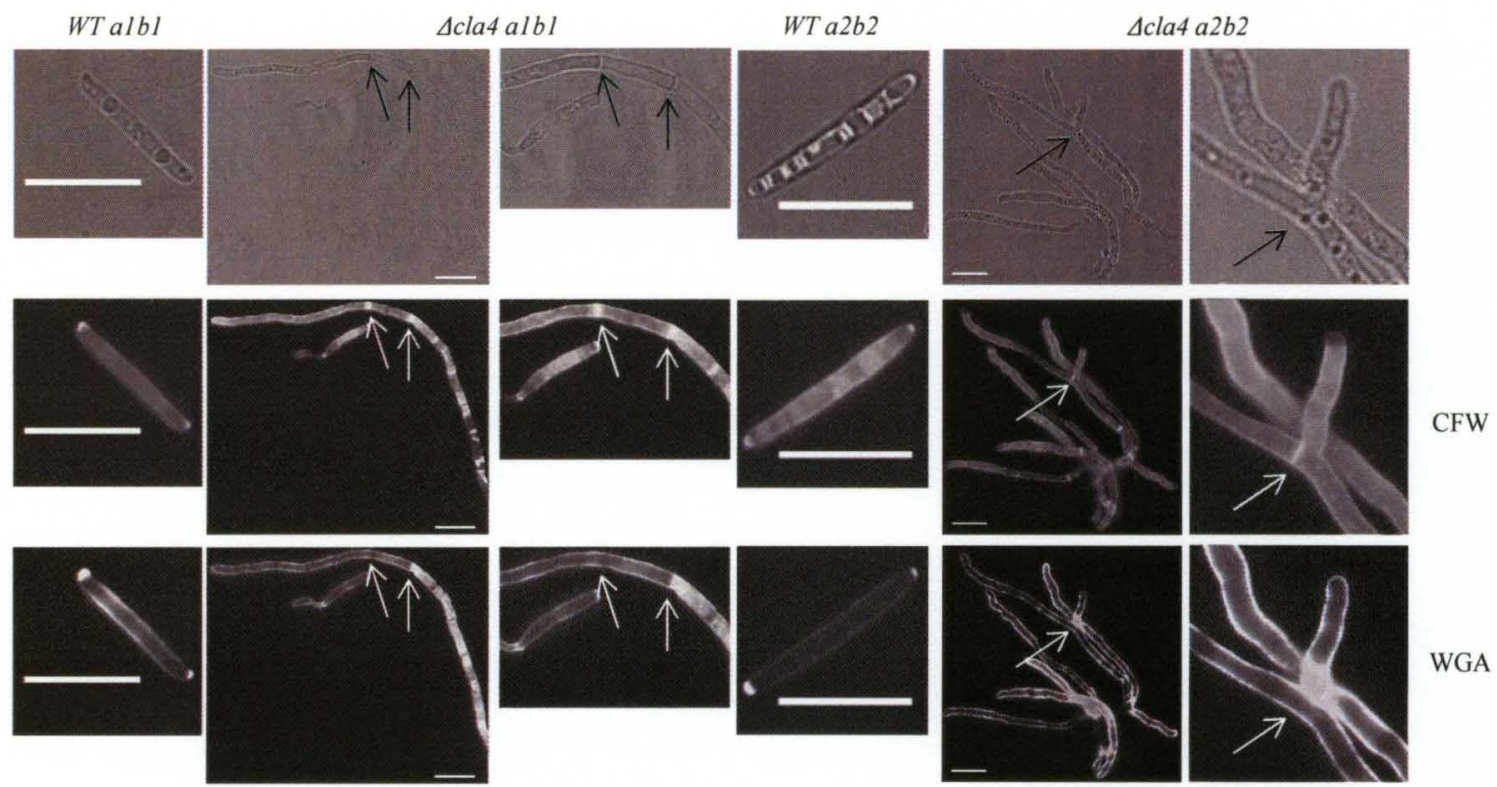

Figure 31: Acla4 strains produce filaments in liquid SLAD and display defects in cell wall localization. $\triangle$ cla 4 cells produce filaments in liquid SLAD while $W T$ cells do not. The alb1 $\triangle$ cla 4 cells exhibit unbranched septated hyphae while the $a 2 b 2 \Delta c l a 4$ cells were highly branched displaying very few septa, with branch points occurring at cross wall septa (See arrows). Both $\Delta$ cla 4 mating-type strains display massive chitin delocalization but no defects in $\beta$-glucan localization. Scale bars, $10 \mu \mathrm{m}$.

media, where chitin was not localized to the polar tip of the cells or with any septa that are formed, but all along the cell wall. The $c l a 4^{\text {Otef }}$ mutant strains, when grown in liquid SLAD, did not differ from the wild type progenitor strains (Fig. 32B).

To explore if the compartments delineated by retraction septa in the alb1 $\Delta c l a 4$ mutant strain contained only a single nucleus or separated multiple nuclei, these cell structures were co-stained with DAPI, CFW, and WGA. The long filaments again exhibited massive chitin delocalization, whereas the $\beta$-glucans localized to the septa correctly (Fig. 32A, see arrows). Nuclear staining with DAPI, indicated a single nucleus in the leading compartment of the albl mutant strain (Fig. 32A, see arrows). Examination of the $a 2 b 2 \Delta c l a 4$ mutant strain indicated a single nucleus contained between randomly interspersed septa (Fig. 32A, see arrows). However, differences between the mating-type backgrounds indicated different responses to liquid low 

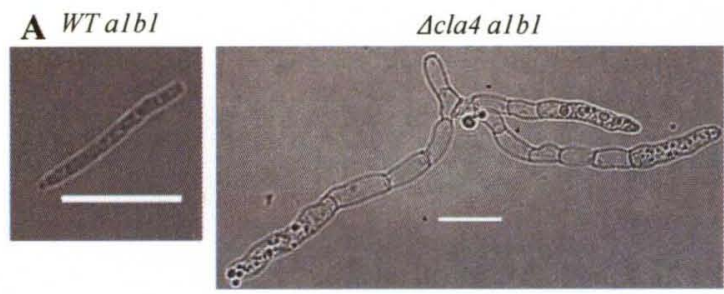

WT $a 2 b 2$
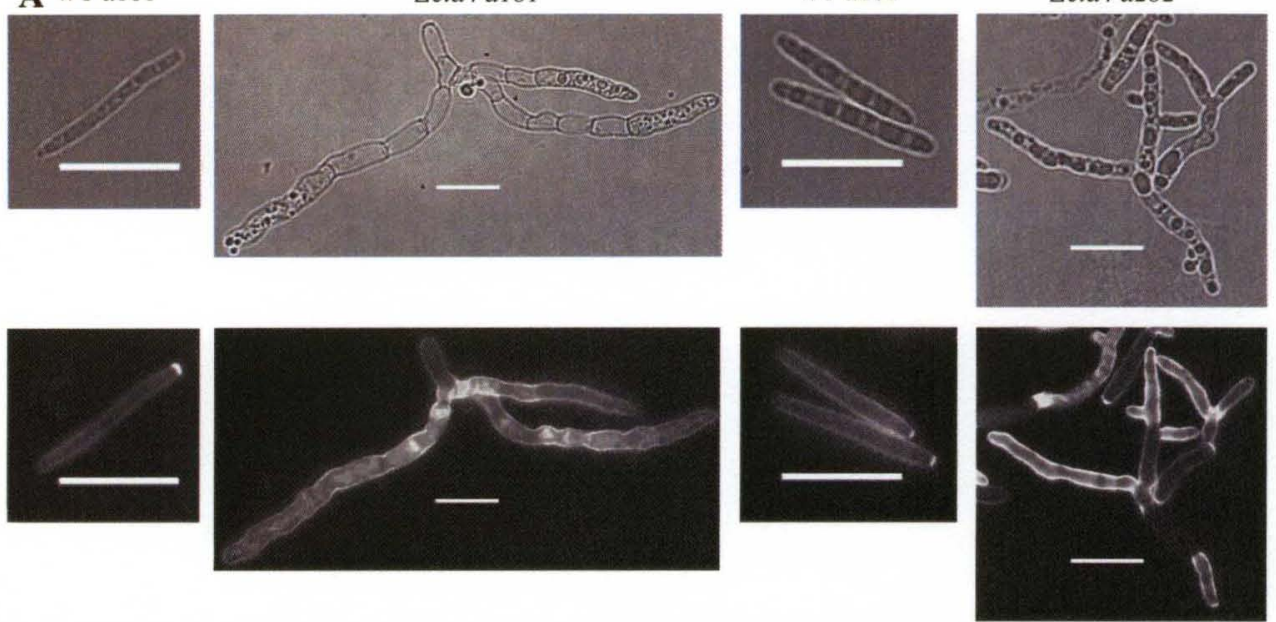

WGA
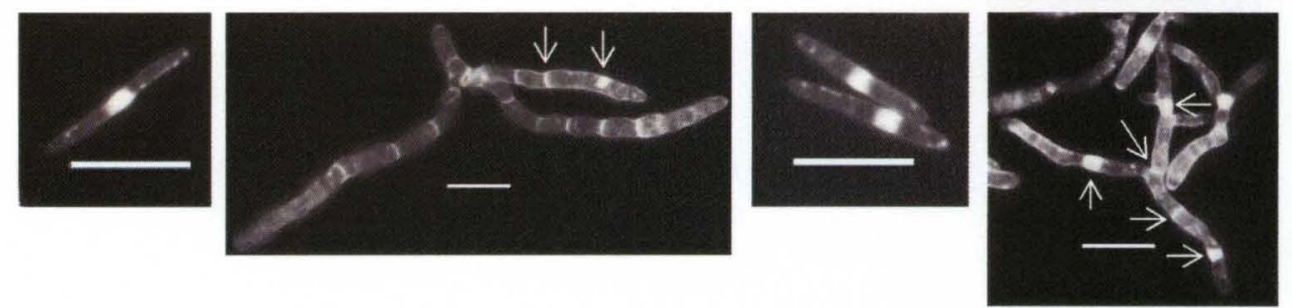

CFW / DAPI

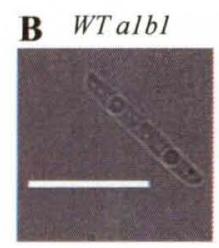

cla $4^{\text {Otef }} a \mathrm{lbl}$
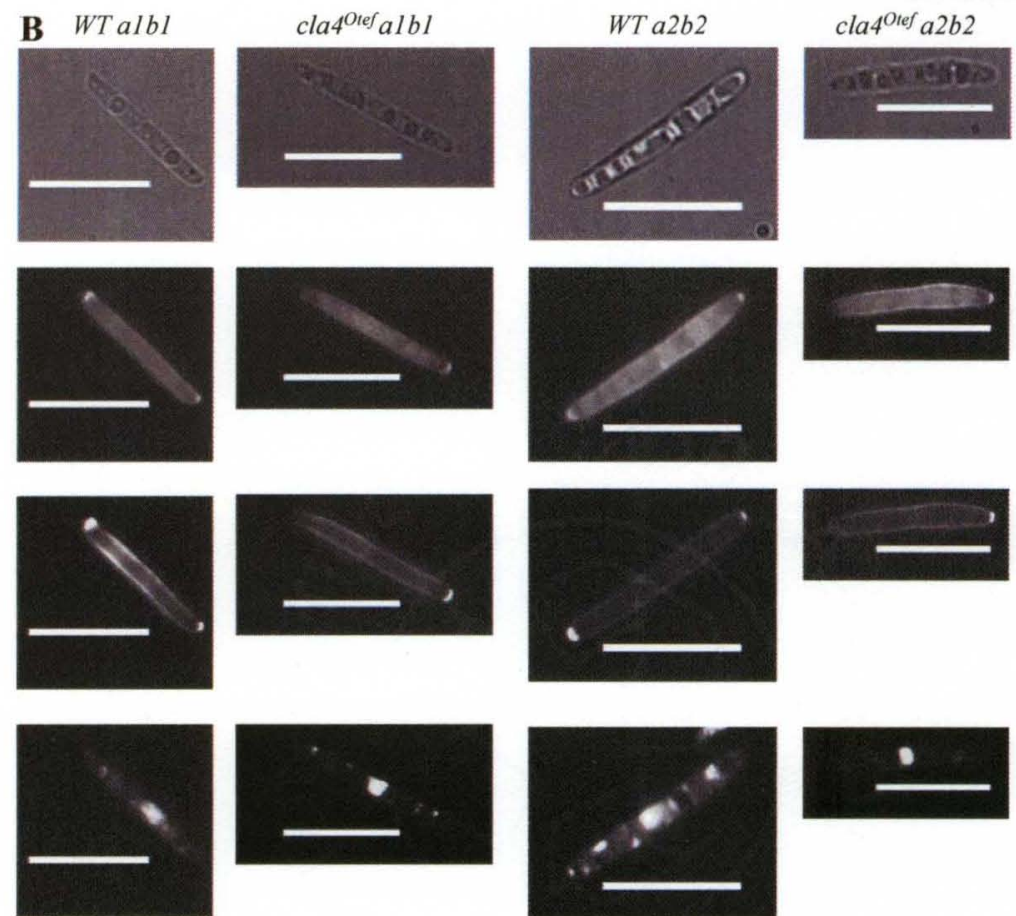

CFW
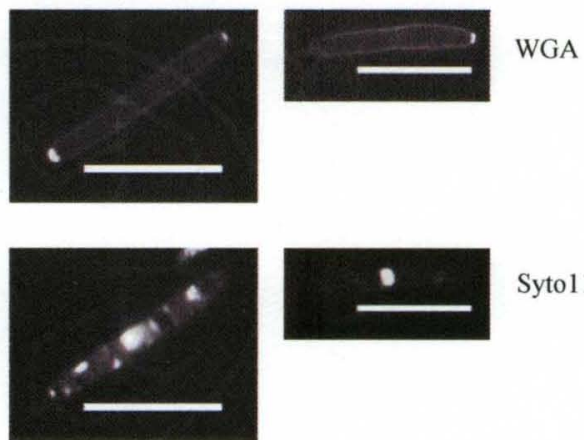

Syto 11

Figure 32: $\Delta$ cla4 strains display mating-type specific effects in liquid SLAD, while cla $^{\text {Otef }}$ strains did not exhibit any defects in cell wall localization and nuclei number. The filaments of the albl $\Delta$ cla 4 cells exhibit a single nucleus and septa confined to the tip of the filament by retraction septa. The $a 2 b 2$ $\triangle$ cla 4 cells exhibited randomly spaced septa that separate the individual nuclei into compartments (A, see arrows). The $c l a 4^{\text {Olef }}$ strains do not display any defects in cell wall localization (stained with CFW and WGA) or nuclear content (stained with Syto11) (B). Scale bars, $10 \mu \mathrm{m}$. 
ammonium conditions. The alb1 $\Delta c l a 4$ mutant strain displayed regularly spaced retraction septa whereas the $a 2 b 2 \Delta c l a 4$ mutant strain displayed few septa, although the septa in both mating-type backgrounds did separate each nucleus into an independent compartment.

\section{$\Delta r a c 1$ is able to form filaments in response to low ammonium conditions,} while overexpression of racl leads to filamentation in rich media. Mahlert, et al. showed that overexpression of $\mathrm{racl}$ promotes filamentous growth, whereas deletion of racl attenuates filamentous growth. The cell morphology of the two racl mutant strains was distinctly different (Fig. 33). The $\Delta \mathrm{racl}$ mutant strain displayed cells that were fatter
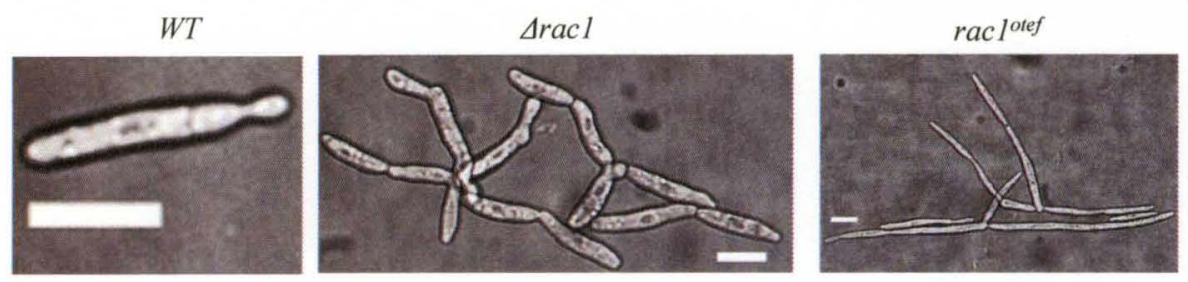

Figure 33: Racl plays a role in cytokinesis and cell morphology. $\Delta \mathrm{racl}$ cells displayed cell separation defects. $\mathrm{racl}^{\text {Otef }}$ cells exhibit a dramatic increase in cell length. Scale bars, $10 \mu \mathrm{m}$.

and failed to separate normally compared to the wild type strain. This phenotype is similar to the phenotype observed for the $\Delta c l a 4$ mutant strains. Overexpression of $r a c 1$ was created by introducing the $\mathrm{racl}^{\text {Otef }}$ construct into the $a l b l$ background strain as previously described. The $\mathrm{racl}^{\text {Otef }}$ mutant strain did form filaments as previously described (Fig. 33 and Mahlert, et al., 2006). With the regulation of filament formation in mind, colony and micro-colony morphology was explored. Deletion of $\mathrm{racl}$ did not affect the morphology of colonies or micro-colonies on YEPS (Fig. 34A and B). Overexpressing racl did lead to a filamentous colony and micro- colony phenotype on YEPS (Fig. 34A and B). In addition, the $\mathrm{racl}^{\text {Otef }}$ mutant strain exhibited directional growth seen previously in the alb1 $\Delta_{810-2832} h \mathrm{sl} 7 \mathrm{smul}{ }^{\text {Otef }}$ mutant strain. 

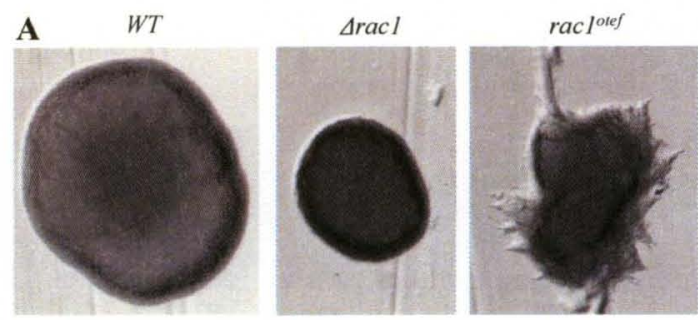

B

WT
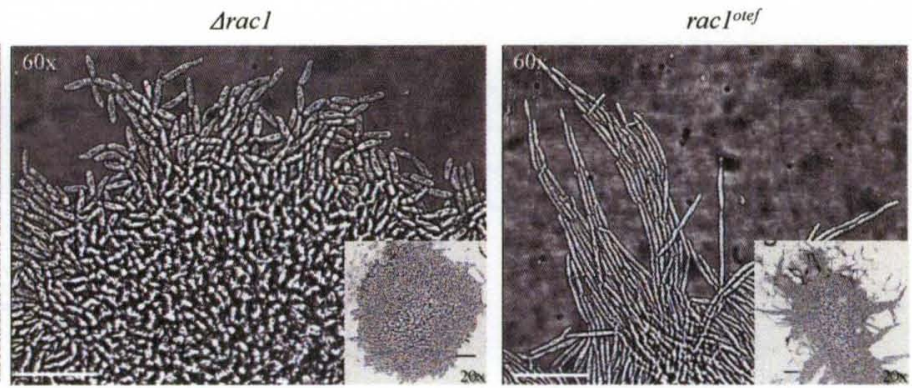

Figure 34: Rac1 mutant strains differ in colony morphology on rich media. The $\Delta \mathrm{racl}$ strain colonies did not display any defects in colony morphology, whereas the $\mathrm{racl}^{\text {Otef }}$ strain exhibited a filamentous colony morphology on rich media (A). The micro-colonies of the $\Delta \mathrm{racl}$ strain did not differ from those of the wild type strains, however the $\mathrm{racl}^{\text {Otef }}$ strain exhibited extremely elongated cells and a "directional growth" phenotype (B). Scale bars, $50 \mu \mathrm{m}-20 \mathrm{x}$ inset image, $40 \mu \mathrm{m}-60 \mathrm{x}$

Under low ammonium conditions, the $\Delta r a c 1$ mutant strain displayed a dramatic reduction in filament formation, whereas the $\mathrm{racl}^{\text {Otef }}$ mutant strain exhibited a dramatic increase in filament formation for both colonies and micro-colonies (Fig. 35A and B). The colonies of the $\mathrm{racl}^{\text {Otef }}$ mutant strain produced larger, thicker filaments than the wild type progenitor strain. Examination of the micro-colonies at 20x magnification revealed elongated filaments, in addition to directional growth (Fig. 35B, inset). At 60x magnification, it became clearer that the long filaments were not highly branched (Fig. 35B). The phenotype was comparable to the phenotype observed in YEPS media, with only the length of the cells increased. The $\Delta \mathrm{racl}$ mutant strain again appeared reduced in the filamentous response to low ammonium when viewed at 20x magnification (Fig. 35B, inset). At 60x magnification, an interesting phenotype was observed; many of the cells were bent or curved in appearance (Fig. 35B). In addition, rounded structures were present at the ends of the majority of cells, while a few cells began to elongate to 
A $\quad W T$
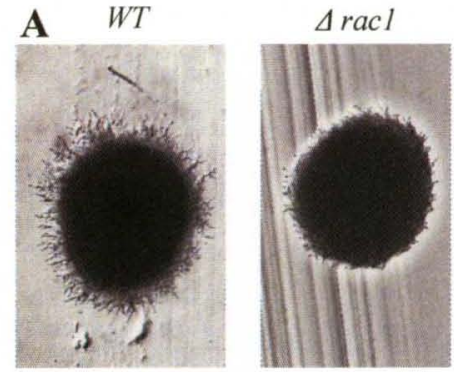

B

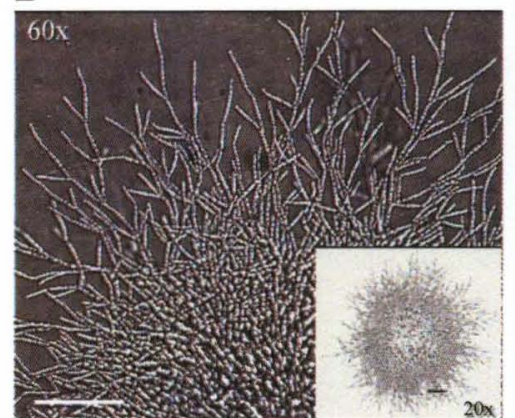

$\triangle \mathrm{racl}$

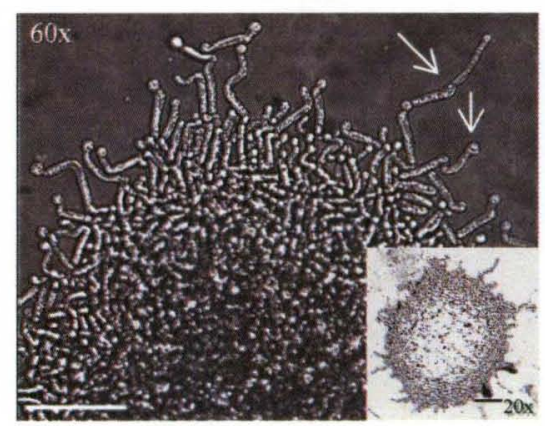

raclotef
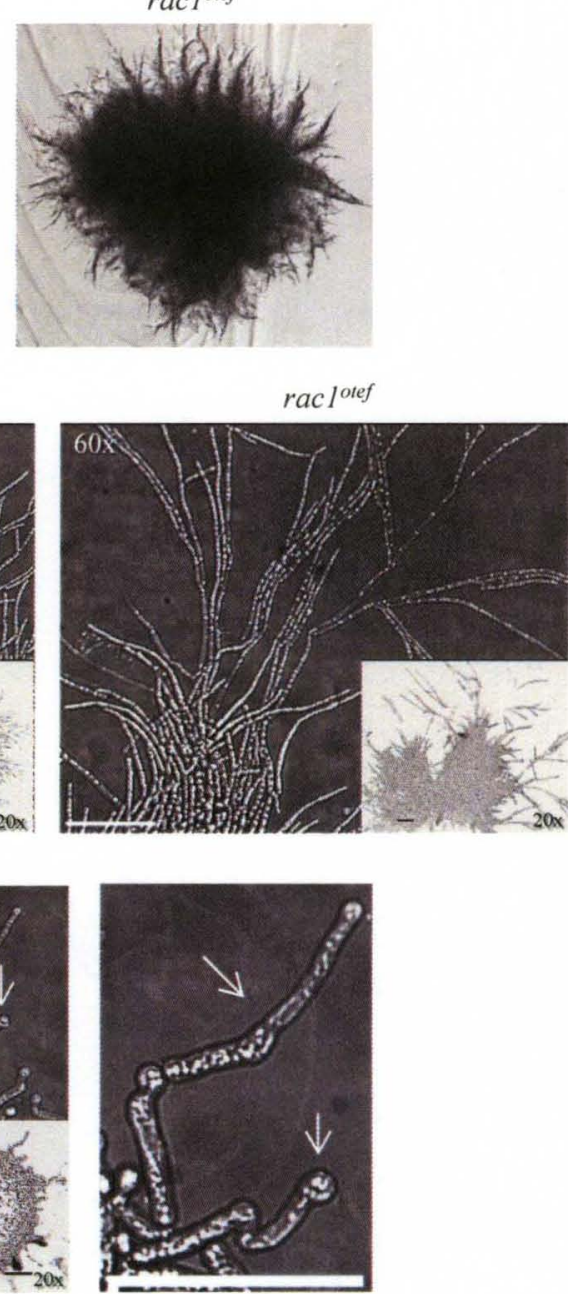

Figure 35: The

filamentous response to SLAD of the Rac1 mutant strains vary compared to wild type strains. The $\Delta r a c l$ strain displays a dramatic reduction in filament formation while the $\mathrm{racl}^{\text {Otef }}$ strain exhibits exaggerated filament formation on SLAD media (A). The $\mathrm{racl}^{\text {Otef }}$ strain was hyper-filamentous when observed in SLAD micro-colonies. The $\Delta r a c l$ strain exhibited bent, shorter, thickened cells that ended with a rounded structure at the apical tip. A few cells were beginning to form filaments (B, see arrows). Scale bars, $50 \mu \mathrm{m}-20 \mathrm{x}$ inset image, $40 \mu \mathrm{m}-60 \mathrm{x}$ image.

potentially form filaments (Fig. 35B, see arrows). The rounded structures are reminiscent of a similar structure observed in a constitutively active $r a c l$ mutant strain $\left(\mathrm{rac}^{Q 61 L}\right)$; however, expression of $\mathrm{rac}^{\text {Q61L }}$ is lethal (Mahlert, et al., 2006).

The ability of the racl mutants to filament in liquid SLAD was examined since I had observed filament formation of the cla 4 mutant strains under theses growth conditions. Interestingly, neither the $\Delta \mathrm{racl}$ nor the $\mathrm{racl}^{\text {Otef }}$ mutant strain formed filaments when grown in liquid SLAD (Fig. 36). Both the $\Delta \mathrm{racl}$ and the $\mathrm{racl}^{\text {Otef }}$ mutant strain displayed a single nucleus when stained with Syto 11 and no cell wall defects were 

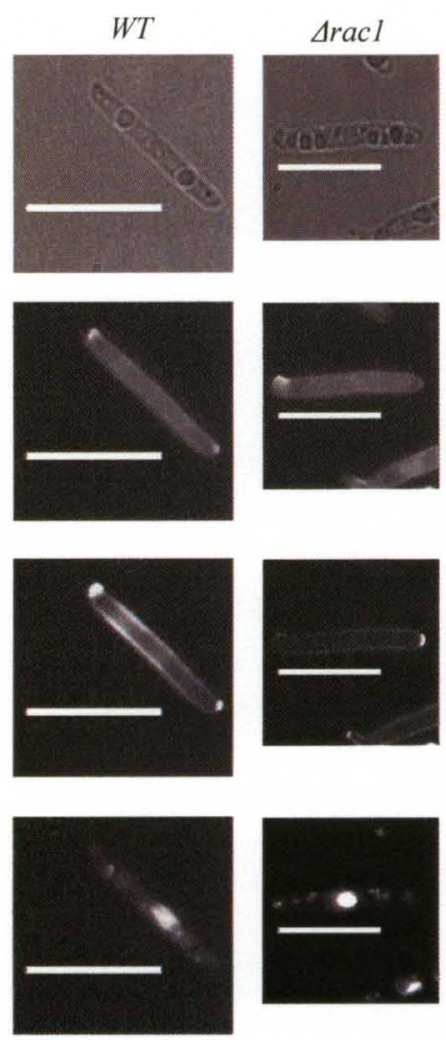
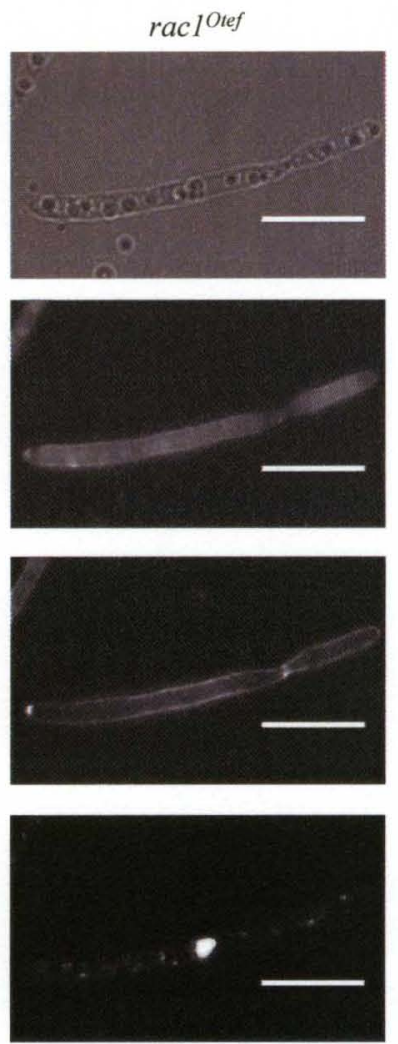

Figure 36: Rac1 mutant strains are unaffected in cell wall localization and nuclei number in

CFW SLAD media. Neither $\triangle \mathrm{racl}$ nor $\mathrm{racl}^{\text {Otef }}$ cells display any defects in cell wall localization WGA or nuclei number on SLAD media. The racl ${ }^{\text {Otef }}$ cells were elongated but did not form filaments in Syto11 SLAD media. Scale bars, $10 \mu \mathrm{m}$.

observed when stained with CFW and WGA, although the $\mathrm{racl}^{\text {Otef }}$ mutant strain was elongated compared to the wild type strain (Fig. 36). This result differs from what was observed in the $\Delta c l a 4$ mutant strains, where the $\Delta c l a 4$ mutant strains were filamentous and display cell separation defects.

Time delay of the filamentous response to low ammonium conditions. The conflicting filamentous phenotypes observed for colonies of the $c l a 4^{\text {Otef }}$ mutant strain and micro-colonies in addition to the dramatic reduction in the filamentous response by the Aracl mutant strain could indicate a potential delay in the filamentous response to low ammonium conditions in these mutant backgrounds. To explore this hypothesis, the cla 4 and $\mathrm{racl}$ mutant strains were streaked onto SLAD media and incubated at $25^{\circ} \mathrm{C}$ for 6 days, and images were gathered at both 3 and 6 days post inoculation (dpi). Comparisons between the wild type strains at both and 3 and 6 dpi indicated no differences in the 
filamentous response between the mating-type backgrounds (Fig. 37A and B). Examination of the cla 4 and $\mathrm{racl}$ mutants indicated temporal effects in the filamentous response. At 3 dpi, the $\Delta c l a 4$ mutant strains displayed mating-type background differences in colony morphology. The albl $\Delta c l a 4$ mutant strain exhibited an increase in filament formation, while the $a 2 b 2 \Delta c l a 4$ mutant strain displayed a decrease in filament formation in comparison to the wild type progenitor strains (Fig. 37A). Though the overall filamentous response was increased for both the $a 1 b 1$ and the $a 2 b 2$ mutant strains, the mating-type specific differences from the wild type strains observed at $3 \mathrm{dpi}$ was maintained at 6 dpi (Fig. 37B). The dramatically elongated filaments exhibited in the alb1 $\Delta c l a 4$ mutant strain at 3dpi, continued to elongate, and by $6 \mathrm{dpi}$, displayed extremely long, single septated hyphae (Fig. 37B). At 3 dpi, the $c l a 4^{\text {Otef }}$ mutant strains displayed similar mating-type background specific phenotypes to those observed in the $\Delta c l a 4$ mutant strains (Fig. 37A). The alb1 mutant strain exhibited an increase, while the $a 2 b 2$ mutant strain exhibited a decrease in filament formation compared to the wild type strains. Though the overall colony morphology was different than the wild type strains at $6 \mathrm{dpi}$, the $a 2 b 2 \mathrm{cla}^{\text {Otef }}$ mutant strain still displayed a decrease in the filamentous response, while for the albl clat $4^{\text {otef }}$ mutant strains overall filament formation was comparable to the wild type strain (Fig. 37B).

Examination of the $\Delta \mathrm{racl}$ and $\mathrm{racl}^{\text {Otef }}$ mutant strains indicated time dependent changes in the filamentous response to SLAD. At, $3 \mathrm{dpi}$, the $\triangle \mathrm{racl}$ mutant strain displayed a dramatic decrease in the ability to form filaments on SLAD compared with the wild type strain (Fig. 37A). However, the colony was not smooth as it was when grown on rich media. The subtle filament formation indicated that $\mathrm{racl}$ dramatically 

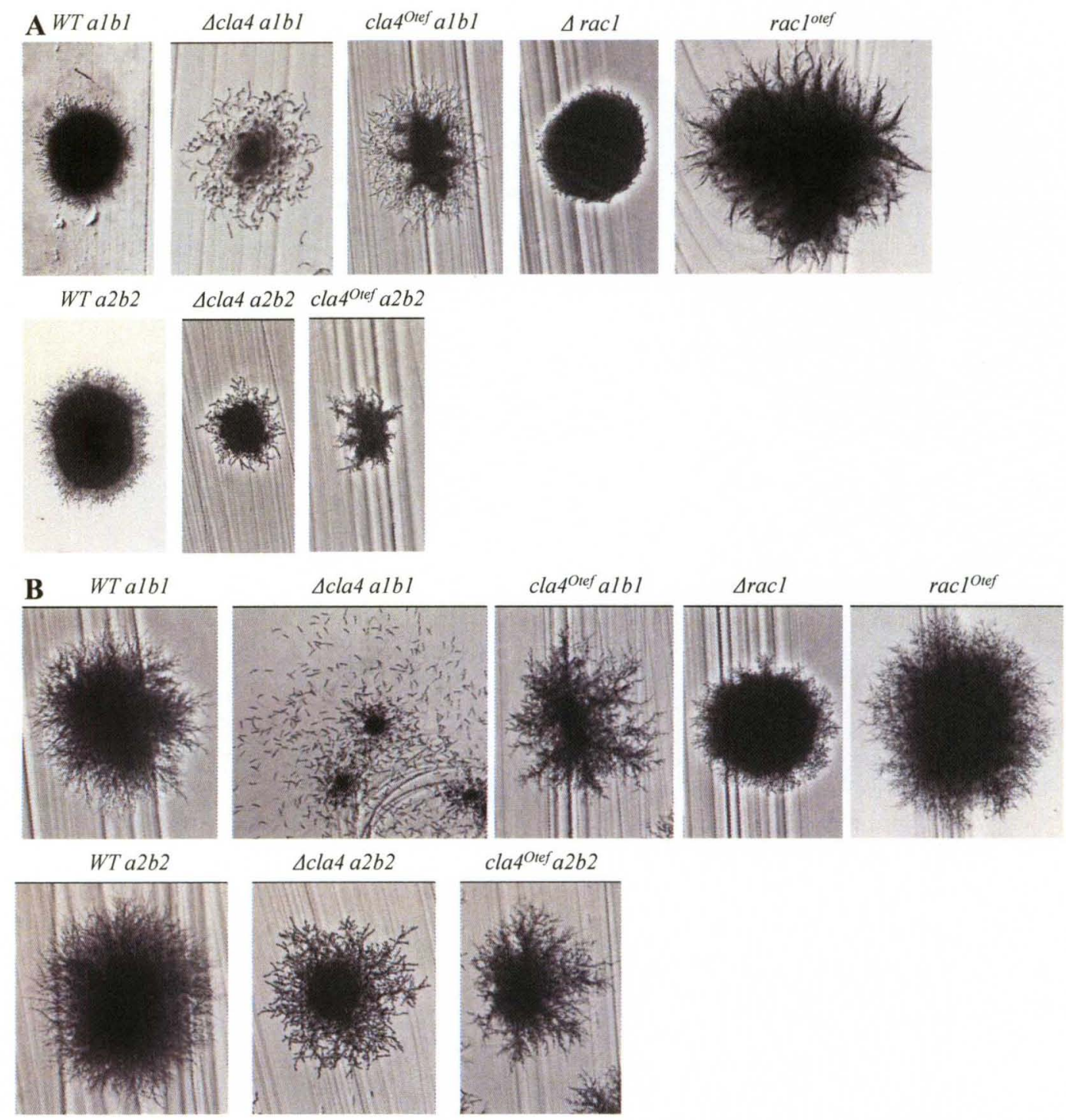

Figure 37: Colony morphology of Cla4 and Rac1 mutant strains grown on SLAD for 3 and 6 days post inoculation (dpi). Examination of Cla4 mutants at 3 dpi indicates that the albl mating-type of both the $\triangle$ cla 4 and $c l a 4^{\text {Otef }}$ mutant strains displayed an increase in the filamentous response compared to wild type strain. The $a 2 b 2 \Delta c l a 4$ and $c l a 4^{\text {Otef }}$ mutant strains were reduced in filament formation compared to wild type strains. The $\Delta \mathrm{racl}$ strain did not form filaments whereas the $\mathrm{racl}^{\text {Otef }}$ strain was increased in filamentation on SLAD at $3 \mathrm{dpi}$ (A). At $6 \mathrm{dpi}$, the alb1 $\Delta$ cla 4 mating-type strain displayed dramatically elongated filaments while the $a 1 b 1$ cla $4^{\text {Otef }}$ mutant strain appeared similar to the wild type strain. The $a 2 b 2 \Delta c l a 4$ and $c l a 4^{\text {Otef }}$ mutant strains were again reduced in filament formation compared to the wild type strain. The $\Delta \mathrm{racl}$ strain began to form filaments, but they were severely reduced compared to the wild type strain. The $\mathrm{racl}^{\text {Otef }}$ strain was still increased in filamentation on SLAD at 6 dpi (B).

reduces, but does not eliminate the ability to form filaments under low ammonium

conditions. This idea was borne out when the $\Delta r a c l$ mutant strain was examined at $6 \mathrm{dpi}$.

Although the mutant did not produce filaments that were comparable to those observed in 
the wild type strain, the $\triangle \mathrm{racl}$ mutant strain did produce filaments in response to SLAD (Fig. 37B). On the other hand, the $r a c 1^{\text {Otef }}$ mutant strain displayed an increase in the filamentous response compared to the wild type at 3 dpi (Fig. 37A). At 6 dpi, the $\mathrm{racl}^{\text {Otef }}$ mutant strain still displayed an increased colony size as well as increased filament formation on SLAD media (Fig. 37B). However, the difference between the $\mathrm{racl}^{\text {Otef }}$ mutant and the wild type strain at $6 \mathrm{dpi}$ is not as dramatic as the difference that is observed at 3 dpi. Overall these results indicate that of these multiple gene products examined, all the mutant strains were able to filament in response to low ammonium conditions. However, the various mutant strains show a delay in the ability to form filaments under low ammonium conditions.

\section{DISCUSSION}

\section{Cla4 plays a role in cell length that is mating-type specific and negatively} regulates the filamentous response to low ammonium conditions. Cla4 is a member of the highly conserved p21-activated protein kinase family. Deletion of cla4 disrupted the ability of $U$. maydis to undergo normal bud formation; subsequently $\Delta c l a 4$ cells undergo elongated growth and divide by fission from a central septum. Nuclear staining indicated that each septated compartment contained a single nucleus. In addition, $\Delta c l a 4$ mutant strains exhibited defects in chitin localization as well as in the ability to form filaments both in the mating reaction and in planta, leading to a decrease in virulence (Leveleki et al., 2004). A significant increase in cell length was observed only in the $a 2 b 2$ mating-type background of $\Delta c l a 4$ mutant strains. A mating-type specific cell length phenotype was also observed in mutants of the gene smul, though $\Delta s m u l$ mutants were decreased in cell length compared to wild type strains (Chapter II). Neither of the 
mutant strains of the albl mating-type background was significantly different compared to the wild type strains, though the difference between the $\Delta c l a 4$ and the $\Delta s m u l a l b 1$ mating-type backgrounds was significant. In S. cerevisiae, Cla4, a target of Cdc42, promotes septin collar formation, subsequently allowing targeting of Swe1 to the bud neck for degradation (Versele and Thorner, 2004). In addition, Cla4 is involved in the hyper-phosphorylation of Swe1, targeting Swe1 for degradation and allowing proper and timely transition from $\mathrm{G}_{2}-\mathrm{M}$. Swe1 monitors septin (actin) filament formation and is stabilized when perturbations occur causing a $\mathrm{G}_{2}$ delay, blocking mitosis (Versele and Thorner, 2004). An effect on cell length was also observed for the strains containing an ectopic gene copy for overexpression of smul or cla4. Interestingly, both the clat $4^{\text {tef }}$ and the $s m u 1^{\text {Otef }}$ albl mutant strains exhibited a comparable increase in cell length, whereas neither $a 2 b 2$ mating-type specific mutant strains were different from the wild type strains or one another. Overall these results present an interesting picture of the relationship between the two PAKs studied in U. maydis. In yeast, both Ste20 and Cla4 play a role in cytoskeletal dynamics and septum formation during cell separation, as well as cell cycle regulation (Cvrčková, et al., 1995; Benton et al., 1997; Eby, et al., 1998; Holly and Blumer, 1999; Höfken and Schiebel, 2002; Versele and Thorner, 2004). It is possible that Cla4 in U. maydis plays a role in cell cycle regulation by targeting Weel for degradation in a $\mathrm{Cdc} 42$ / Rac1 dependent manner, thereby regulating a critical step in $\mathrm{G}_{2}$ $\mathrm{M}$ transition and cytokinesis. If so, this would be in addition to the activity of Cla4 in polar growth, though more work needs to be done to clarify this potential role.

$\Delta c l a 4$ mutant strains are unable to form filaments in the mating reaction, though they are capable of cell recognition, cell fusion, and filament formation in planta 
(Leveleki, et al., 2004). Conversely, the $\Delta c l a 4$ mutant strains exhibited a filamentous response on low ammonium conditions including liquid SLAD, again with phenotypes that varied according to mating-type. In addition, both $\Delta c l a 4$ mutant strains displayed defects in chitin but not $\beta$-glucan localization. Overexpression of cla 4 resulted in an increase in the filamentous response to SLAD in the $a l b l$ mating-type background; however the $a 2 b 2 \mathrm{cla} 4^{\text {Otef }}$ mutant strain exhibited a decrease in the filamentous response to SLAD. Interestingly, the $c l a 4^{\text {Otef }}$ mutant strain micro-colonies displayed a dramatic decrease in the filamentous response to SLAD independent of mating-type background. Overall the role of Cla4 in the filamentous response to low ammonium conditions is complicated. Cla4 potentially plays a role in polar growth and proper bud formation in addition to possible cell cycle effects. Absence of Cla4 may not directly cause the filamentous response, but rather delay the proper cell cycle timing allowing other mechanisms regulating the filamentous response to act, creating elongated cells.

racl mutant strains are able to form filaments in response to low ammonium conditions. Cla4 is an effector of the Rho / Rac-like GTPase, Rac1, and regulates polar growth in U. maydis (Mahlert, et al., 2006). Deletion of racl leads to attenuation of polar growth, with the cells appearing very similar to those of $\Delta$ cla 4 mutant strains, being fatter and failing to separate through normal mother daughter septa. This indicates that Racl potentially localizes Cla4 to the bud site allowing proper formation of the septin ring, ultimately allowing for proper $\mathrm{G}_{2}-\mathrm{M}$ transition. Conversely, overexpression of $\mathrm{racl}$ leads to dramatic cell elongation and a polar growth pattern similar to Wee1 overexpression strains (Sgarlata and Pérez-Martín, 2005). Overexpression of Rac1 may sequester Cla4 from the septin ring to the growing polar tip of the bud, delaying Wee1 
phosphorylation and subsequent degradation extending $\mathrm{G}_{2}$, while concomitantly causing polar growth. Equally, the over abundance of Rac1 may simultaneously promote normal cell cycle propagation and polar growth, whereas polar growth is not as dramatic in the wild type strains due to lower stoichiometric levels of Racl. The $\mathrm{racl}{ }^{\text {Otef }}$ mutant strain colony and micro-colony results are very similar to the filamentous and directional growth responses observed in the $a 1 b 1 \Delta_{810-2832} h \mathrm{sl} 7 \mathrm{smu} 1^{\text {Otef }}$ mutant strain indicating a shared role in cell elongation and potentially in cell cycle regulation (Chapter II). Under conditions of low ammonium, the $\Delta \mathrm{racl}$ mutant strain exhibited a marked reduction in the ability to form filaments, though it was observed primarily in micro-colonies that filaments were beginning to form, indicating a potential delay in the filamentous response to low ammonium. The cell morphology of the $\Delta \mathrm{racl}$ mutant strain exhibited two striking features, curved or bent cells and a bulbous structure at the end of the cells. A similar bent phenotype was observed in $\Delta c l a 4$ mutant strains that form a centrally located septum and separate by contraction, which can result in a bending of daughter cells. Yet the bent phenotype observed in the $\Delta \mathrm{racl}$ mutant strain does not appear to be due to new daughter cells forming from a central septum, but rather the cell itself contorting. Even though the $\triangle \mathrm{racl}$ mutant strain was viable in SLAD media, the rounded structure at the ends of the cells was reminiscent of the constitutively active and lethal form of $\mathrm{racl}$, $\operatorname{Racl}^{\mathrm{Q} 61 \mathrm{~L}}$ (Leveleki, et al., 2004; Mahlert, et al., 2006). The racl ${ }^{\text {Otef }}$ mutant strain produced large thick filaments, similar to the dramatically thickened colony morphology observed in a $\Delta u m p 1 / \Delta u m p 2$ double mutant (Smith, et al., 2003). In addition, the $\mathrm{racl}^{\text {Otef }}$ mutant strain exhibited a directional, hyper-filamentous phenotype on microcolonies. However in liquid SLAD, the $\mathrm{racl}^{\text {Otef }}$ mutant strain, though elongated 
compared to wild type strains, failed to form filaments differing dramatically from phenotypes observed for either the $\Delta_{810-2832} h s l 7$ smul ${ }^{\text {Otef }}$ or the $\Delta c l a 4$ mutant strains. These results indicate that the phenotypes observed in low ammonium conditions in the $\Delta_{810-2832} h s l 7 s m u I^{\text {Otef }}$ or the $\Delta c l a 4$ mutant strains are independent of Racl. However, the $\Delta r a c l$ mutant strains are able to form filaments, albeit reduced dramatically, indicating that Rac1 plays some role in the filamentous response (see below), but is not absolutely required in all circumstances.

\section{Time effects on the filamentous response to low ammonium conditions.}

Responses to nutrient conditions are complex and vary from nutrient to nutrient. $U$. maydis undergoes a morphological transition from a budding phenotype to a filamentous phenotype in response to low ammonium conditions, growth on lipids as the carbon source, or acidic pH (Ruiz-Herrera, et al., 1995; Smith et al., 2003; Klose, et al., 2004). Ammonium is the preferred nitrogen source for many fungi and under low ammonium conditions $(<100 \mu \mathrm{m})$ the colonies of $U$. maydis strains produce a filamentous colony phenotype. The extent of the filamentous response is time dependent, with a greater and greater production of filaments over a greater duration on low ammonium conditions. At $3 \mathrm{dpi}$, several of the previously examined mutant strains exhibited differences compared to the wild type strains in the ability to form filaments on SLAD media, most dramatically the $\Delta \mathrm{racl}$ mutant strain, which failed to form filaments. At $6 \mathrm{dpi}$, the increase in the filamentous response of the $\mathrm{cla}^{\text {Otef }}$ and $\mathrm{racl}^{\text {Otef }}$ in comparison to the wildtype strain had diminished. Conversely, the $\Delta \mathrm{racl}$ mutant strain produced filaments, though they were severely reduced in comparison to those of wild type strains. These results contradict the hypothesis of $\mathrm{racl}$ acting as the master regulator of the filamentous 
response. The deletion of $\mathrm{racl}$ appears to delay the ability of the cells to produce filaments on SLAD media indicating other players are involved in the filamentous response to SLAD media or a parallel pathway regulating the filamentous response. Moreover, the delay in the onset of the filamentous response by $\Delta \mathrm{racl}$ mutant strain could be due to changes in the media independent of low ammonium (i.e. glucose levels or secondary metabolite production) triggering the filamentous response. Evidence in Aspergillus niger indicates that spatial recognition triggers either expression of three genes involved in nitrate reduction at the colony edge or nitrogen metabolic repressor at the colony center (Levin et al., 2007). This spatial gene expression appears to be independent of nitrate concentrations, as well as carbon source. In addition, the expression genes involved in nitrate reduction remained constant upon transfer to fresh media, indicating that accumulation of secondary metabolites does not seem to modulate gene expression. Quorum sensing, a potential explanation for spatial gene expression, has been observed in C. albicans where cell density negatively affects biofilm production and pathogenesis (Hogan, 2006). Though not yet demonstrated, a similar process could explain spatial and temporal behaviors of $U$. maydis.

Conclusions. Rho / Rac GTPases have been implicated in regulating cell polarity, cytoskeletal organization, and cytokinesis (Nakano, et al., 1997; Cabib, et al., 1998; Madden and Snyder, 1998; Lengeler, et al., 2000; Tolliday, et al., 2002; Nelson, 2003; Bassilana and Arkowitz, 2006; Mahlert et al., 2006). PAKs have been identified as potential downstream effectors of the Rho / Rac GTPases, contributing to many of the GTPase specific roles (Roberts and Fink, 1994; Cvrčková, et al., 1995; Marcus, et al., 1995; Gilbreth, et al., 1996 and 1998; Leberer, et al., 1997; Eby, et al., 1998; Holly and 
Blumer, 1999; Fujita, et al., 1999; Bao, et al., 2001; Höfken and Schiebel, 2002; Qyang, et al., 2002; Wiley et al., 2003; Leveleki, et al., 2004; Smith, et al., 2004; Heinrich, et al., 2007; Böhmer, et al., 2008). In U. maydis, Rac1 establishes polar growth, while Cdc42 regulates cell separation, and Rhol is required for cell polarity and cytokinesis (Mahlert et al., 2006; Pham et al., 2009). Cla4 has been identified as a downstream effector of Rac1, while downstream effectors of $\mathrm{Cdc} 42$ and Rho1 have yet to be identified, although Cla4 is a potential prospect (Pham, et al., 2009). Cdc42, through its GEF, Don1, is required for formation of the second septum allowing proper mother daughter cell separation (Mahlert et al., 2006). Cdc42 localization and activity at the second septum requires the activity of the PAK Don3 (Böhmer et al., 2008). Cdc42, which also localizes to the primary septum, is dispensable for polarized growth and filament formation (Mahlert et al., 2006). However, it is interesting to speculate the role of Cdc42 when localized to the primary septum. On the other hand, Rac1 plays a role in polar growth and normal bud formation by its localization to the polar tip, potentially through Cdc24, the Rac1-dependent GEF. Rac1 activity promotes localized cell wall formation in a Cla4 dependent manner (Mahlert et al., 2006). The $\Delta$ cla4 mutant strains display a dramatic disruption in chitin deposition; however the $\Delta r a c l$ mutant strain did not display any defects in chitin deposition. Though both Cla4 and Rac1are involved in polar growth, the role of Cla4 in chitin deposition is independent of Rac1. Ultimately, Rac1 and $\mathrm{Cdc} 42$ have separate roles in $U$. maydis: Rac1, through Cla4, regulates septin apparatus assembly necessary for bud formation, and then promotes polarized cell growth while $\mathrm{Cdc} 42$ is required to promote mother daughter cell separation, but is dispensable for bud formation and polar growth (Mahlert, et al., 2006). Thus it appears that Rac1, 
through Cla4, acts as the master regulator of filament formation (Mahlert, et al., 2006; Pham, et al., 2009). However, very little work has been performed on Rac1, Cdc42, Rho1, and Cla4 under conditions of low ammonium. In this study, I provide evidence that $\mathrm{Cla} 4$ plays a role in the filamentous response to low ammonium conditions. In addition, Rac1 may not be the only upstream activator of Cla4. Moreover, neither Cla4 nor Rac1 are essential in the ability to form filaments under conditions of low ammonium, although the ability to form filaments is greatly reduced in racl deletion strains.

When grown in rich conditions, the $\Delta c l a 4$ mutant cells are morphologically different from the cells of the disrupted U. maydis PAK-like Ste20 homologue, Smu1. $\Delta c l a 4$ cells were septated, containing several independent nuclei having failed to correctly separate, unlike the $\Delta s m u 1$ mutant strains which are affected only in cell length (Fig. 5, Chapter II). These results are consistent with the observed roles of Cla4 in cytokinesis and polar growth in S. cerevisiae and C. albicans (Leberer, et al., 1997; Benton et al., 1997). In addition, the phenotypes observed in the $\Delta c l a 4$ were similar to those exhibited in Cdk1, Cdk5, and Weel mutants, indicating that Cla4 may play a role in cell cycle similar to Cla4 from $S$. cerevisiae (Asano, et al., 2005; Sgarlata and PérezMartín, 2005; Castillo-Lluva et al., 2007). Cell length measures of the $\Delta c l a 4$ mutant strains support this idea. An increase in cell length could indicate a delay in the $\mathrm{G}_{2}-\mathrm{M}$ transition. Additional support was observed in low ammonium conditions where the $\triangle c l a 4$ mutant strains increase filament formation in a mating-type specific manner. This may indicate that though Cla4 is necessary for filament formation in rich conditions, it is dispensable in conditions of low ammonium. Another possibility is that deletion of cla4 
indirectly promotes filamentous growth by failing to promote cell separation (via binding to Rac1), creating a cell cycle delay, while additional pathways are promoting increased polar growth leading to the filamentous response. The role Racl plays in the filamentous response to low ammonium conditions is more complicated. Deletion of $\mathrm{racl}$ dramatically delays filament formation, but does not eliminate the ability to form filaments. Conversely, overexpression of $\mathrm{racl}$ increased the filamentous response; however the $\mathrm{racl}^{\text {Otef }}$ mutant strain did not exhibit filament formation when grown in liquid SLAD, though the mutant cells were elongated. The exaggeration of the filamentous response in the $\Delta c l a 4$ mutant cells, along with the lack of such a response in either $\mathrm{racl}$ mutant strain suggest that the role Cla4 plays in the filamentous response to low ammonium conditions may be independent of Rac1. These results imply that Cla4 may not be the only downstream effector of Rac1, or conversely, Cla4 may have other upstream activators regulating septin assembly and proper cell separation. Potential candidates include $\mathrm{Cdc} 42$ and Rho1. 


\section{CHAPTER IV}

\section{GENERAL DISCUSSION}

Dimorphic fungi undergo a process of yeast-like to hyphal transition in response to nutrient, mating, and/or host cues. Studying how these fungi respond to this myriad of cues provides insight into intracellular signaling in response to extracellular signals. Dimorphic fungi provide an excellent model to study the regulatory pathways associated with cellular morphogenesis due to their ease of genetic manipulation. A complex network of signaling molecules and pathways regulate many essential cellular processes including cell cycle, cytoskeletal organization, cell polarity, cellular morphology, cytokinesis and hyphal development. Exploration of these pathways has identified several proteins that are highly conserved from fungi all the way to humans, as well as the conservation of these pathways as distinct units.

The Rho/Rac family of GTP-binding proteins (GTPases) regulates many of the functions involved in cell growth, development, mating, and differentiation (Nakano, et al., 1997; Cabib, et al., 1998; Madden and Snyder, 1998; Lengeler, et al., 2000; Tolliday, et al., 2002; Nelson, 2003; Leveleki et al., 2004; Mahlert et al., 2006; Pham, et al., 2009). The activity of these proteins is regulated temporally and spatially by an ever growing group of accessory proteins, giving rise to specific landmark effects and subsequent development. Guanine exchange factors (GEFs) are one subset of these accessory proteins and are involved not only in the activation of the GTPases, but in their cellular 
localization (Ziman, et al., 1993; Gulli and Peter, 2001; Weinzierl, et al., 2002; Coll, et al., 2003; Mahlert et al., 2006; Schink and Bölker, 2009). In addition, GEFs regulate GTPase specificity for downstream substrates, defining precise processes. In U. maydis, three highly conserved Rho/Rac GTPases, Cdc42, Rac1, and Rho1 have been implicated in cell separation, bud formation, cell polarity, and polar growth (Weinzierl, et al., 2002; Leveleki, et al., 2004; Mahlert, et al., 2006; Böhmer, et al., 2008; Pham, et al., 2009). Cdc42 activity requires the specific GEF activity of Don1 while it is proposed that Rac1 requires the GEF activity of Cdc24 (Mahlert, et al., 2006; Castillo-Lluva, et al., 2007; Alvarez-Tabarés and Pérez-Martín, et al., 2008; Hlubek, et al., 2008). Hlubek, et al. (2008) indicated that a single amino acid substitution in Rac1 confers Cdc42-specific activities to Rac1. The amino acid substitution promotes these activities by allowing Rac1 to interact with the Cdc42-specific GEF, Don1. A Rho1-specific GEF has yet to be identified, though $\mathrm{Cdc} 24$ has been implicated through in vitro interaction analyses (Pham et al., 2009).

Many functions of the GTPases depend on the highly conserved family of p-21 activated kinases (PAKs) as downstream effectors (Roberts and Fink, 1994; Cvrčková, et al., 1995; Marcus, et al., 1995; Gilbreth, et al., 1996 and 1998; Leberer, et al., 1997; Eby, et al., 1998; Holly and Blumer, 1999; Fujita, et al., 1999; Bao, et al., 2001; Höfken and Schiebel, 2002; Qyang, et al., 2002; Wiley et al., 2003; Leveleki, et al., 2004; Smith, et al., 2004; Heinrich, et al., 2007; Böhmer, et al., 2008). Two specific PAKs, Cla4 and Ste20, have been identified and studied, and although loss of either PAK usually does not result in a lethal phenotype, loss of both PAKs is lethal (Roberts and Fink, 1994; Cvrčková, et al., 1995; Marcus, et al., 1995; Gilbreth, et al., 1996 and 1998; Leberer, et 
al., 1997; Eby, et al., 1998; Holly and Blumer, 1999; Fujita, et al., 1999; Bao, et al., 2001; Höfken and Schiebel, 2002; Qyang, et al., 2002; Wiley et al., 2003; Leveleki, et al., 2004; Smith, et al., 2004; Heinrich, et al., 2007; Böhmer, et al., 2008), indicating that although they regulate separate processes, they share at least one essential function (Cvrčková, et al., 1995). Cla4 and Ste20 homologues have been identified and studied in U. maydis (Leveleki, et al., 2004; Smith, et al., 2004). Cla4 appears to be an effector of Racl, regulating cell separation and polarity, while Smu1 is involved in mating pheromone expression and virulence (Leveleki, et al., 2004; Smith, et al., 2004).

PAK activity is also dependent upon several proteins in addition to Rho/Rac GTPases (Lengeler et al., 2000; García-Pedrajas, et al., 2008). Hs17, a member of the conserved protein-arginine methyltransferase family of proteins, is known to interact with the Ste20 homologues and plays a role in cell cycle regulation, cell morphogenesis, and response to several environmental conditions (Ma, et al., 1996; Gilbreth, et al., 1996 and 1998; Pollack, et al., 1999; Fujita et al., 1999; Bao, et al., 2001). In addition, Hsl7 is known to regulate cell cycle progression in a Wee1 dependent manner (Ma, et al., 1996; Gilbreth, et al., 1998; Shulewitz, et al., 1999; Yamada, et al., 2004; Asano, et al., 2005). In S. cerevisiae, Hsl7 targets Swe1 (Wee1 homologue) for degradation by co-localizing Swel to the bud neck with Hsl1, allowing for hyper-phosphorylation via $\mathrm{Cdc} 5$ and subsequent degradation, promoting $\mathrm{G}_{2}-\mathrm{M}$ transition (Cid, et al., 2001; La Valle and Wittenberg, 2001; Asano, et al., 2005).

I was able to demonstrate in $U$. maydis that Smul, in addition to regulating the expression of mating pheromone and virulence, positively regulates the filamentous response to low ammonium conditions. Moreover, Smul affects cell length. Hs17 plays 
a role in cell length and the filamentous response to low ammonium conditions, yet opposite to the role of Smul. In addition, Hsl7 interacts with Smul in yeast two-hybrid analyses, indicating that Hsl7 may play a role in regulating Smul activity. Fujita, et al. (1999) demonstrated that Hsl7 interacts with Ste20 both in vitro and in vivo, regulating the filamentous response pathway to low ammonium. Genetic analysis indicates that Hsl7 is upstream of Ste20 and the action of Hsl7 upon Ste20 is negative. Deletion of Hsl7 leads to a filamentous response in nitrogen rich conditions that can be alleviated by concomitant deletion of Ste20, while overexpression of $h s l 7$ attenuates the filamentous response to low ammonium, a phenotype that can be rescued by co-overexpressing ste 20 (Fujita, et al., 1999). The disruption of smul does not alleviate $\Delta_{810-2832} h s l 7$ associated phenotypes, nor does the latter deletion rescue $\Delta s m u l$ effects on mating response or virulence. This points to an alternate hypothesis posed in S. cerevisiae, in which Hsl7 regulates the filamentous response in a Swe1-dependent manner independent of Ste20 (La Valle and Wittenberg, 2001). Deletion of $h s l 7$ creates a delay in the $\mathrm{G}_{2}-\mathrm{M}$ transition, causing cell elongation and eases the ability of the cells to promote the filamentous response. It is possible that a similar process occurs in U. maydis, in which $\mathrm{Hsl} 7$ would act on cell cycle regulation, while Smul plays a role in the filamentous response pathway. This is supported by data indicating that overexpressing $s m u l$ while depleting $h s l 7$ leads to an exacerbation of $\Delta_{810-2832} h s l 7$-associated phenotypes, increasing cell length and the filamentous response to low ammonium. Remarkably, mutant strains lacking $h s l 7$ while simultaneously overexpressing $s m u l$ were able to develop filaments in liquid SLAD media. In this study, the only other strains that produced filaments in liquid SLAD were those lacking cla4. 
The PAK homolog, Cla4, differs from Ste20 homologues in one major regard, Cla4 homologues contains a pleckstrin homology $(\mathrm{PH})$ domain, while Ste20 homologues do not. This domain allows Cla4 to bind to the membrane and localize to polar growth sites as well as regulate cell morphogenesis and mitotic exit (Wild, et al., 2004). In $U$. maydis, Cla4 has been identified as the downstream effector of Rac1, regulating bud formation and morphology and polar growth (Leveleki, et al., 2004; Mahlert, et al., 2006). Moreover, deletion of either cla 4 or racl eliminates b-dependent filamentation. However, on SLAD media, $\Delta c l a 4$ strains were still able to produce filaments, being the only other mutant strain to filament in liquid SLAD outside of the $\Delta_{810-2832} h s l 7 \mathrm{smul} 1^{\text {Otef }}$ strains. Interestingly this filamentous response to SLAD appears to be independent of Racl activity. Deletion of $\mathrm{racl}$ created a dramatic delay in the filament formation, while overexpression of racl leads to hyphal growth. Yet, neither mutant strain formed filaments in liquid SLAD. It could be that Cla4 limits the filamentous response to low ammonium conditions by either sequestering a component in the filamentous response pathway (potentially Cdc42 or PAK/MAPK activator) or by promoting bud emergence and normal cell cycle progression preventing cell elongation and hyphal growth. $S$. cerevisiae Cla4 binds Cdc42 promoting septin ring formation and proper bud emergence. Subsequently Cla4 is involved in the hyper-phosphorylation of Swe1 promoting $\mathrm{G}_{2}-\mathrm{M}$ transition (Versele and Thorner, 2004; Asano, et al., 2005). It is interesting that the two PAKs, Cla4 and Smu1, have opposite roles in the filamentous response pathway, where deletion of cla 4 alone or overexpressing $s m u l$ in a $\Delta_{810-2832} h s l 7$ background, leads to a hyper-filamentous response to low ammonium. 
In S. cerevisiae, deletion of both ste20 and cla4 is lethal, indicating that Ste20 and Cla4 share at least one essential function (Cvrčková, et al., 1995). The overlapping role appears to be in actin organization and cell separation (Cvrčková, et al., 1995; Eby, et al., 1998; Höfken and Schiebel, 2002; Kadota, et al., 2004). However, studies by Keniry and Sprague (2003) indicates that different subdomains of Ste20 and Cla4 are functionally separate and governed by different factors. Threonine 818 promotes Ste 20 activity in the HOG and pheromone response pathways, whereas two different regions direct invasive growth. Subdomains III to $\mathrm{X}$ allow the Cla4-specific function for viability in a $\Delta c \ln l$, $\Delta c \ln 2, \Delta c l a 4$ strain. It is possible that a similar form of overlap occurs between Cla4 and Smu1 in U. maydis. They share similar responsibility in mating and subsequent pathogenic development, though Smu1 is required for proper pheromone based signaling, while Cla4 is dispensable for normal pheromone response but necessary for subsequent b-dependent development. Additionally, they both have a role in the filamentous response to low ammonium, yet they are opposite in how they regulate the response. Cla4 appears to regulate the filamentous response, potentially through promoting bud formation and cell separation. The role of Smul is more difficult to understand. Overexpression of smul promotes increased filamentation to SLAD, but only upon simultaneous disruption of $h s l 7$ do I observe the hyper-filamentous response to low ammonium. This particular set of circumstances points to additional players that one or both, Smul and Hsl7, act upon in regulating cell morphogenesis.

Ultimately, both Smu1 and Cla4 are involved in regulating the filamentous response to low ammonium conditions. Here I have demonstrated that, although absence of Cla4 or overabundance of Smu1, with concomitant Hsl7 absence, is not lethal to $U$. 
maydis, either genetic configuration results in a hyper-filamentous response to low ammonium. The interaction between Hsl7 and Smul appears to be a convergence of independent roles creating the exacerbated response. The lack of a clear genetic interaction between Hsl7 and Smul points to the involvement of the two proteins in separate pathways that culminate in regulating the filamentous response to SLAD. It is possible that $\mathrm{Hsl} 7$ plays a role in cell cycle regulation, promoting $\mathrm{G}_{2}-\mathrm{M}$ transition as in $S$. cerevisiae, which would explain the increased cell length and increased filamentous response in SLAD. In addition, Smul could play a role in the filamentous response to SLAD, potentially though the MAPK pathway. The delay in $\mathrm{G}_{2}-\mathrm{M}$ transition created by removing Hs17 primes the cells for the filamentous response to low ammonium, while simultaneous overexpression of Smul potentially leads to hyper-filamentation. This hypothesis allows for the fact that the concurrent disruption of both $h s l 7$ and $s m u l$ does not alleviate any phenotypes associated with either single gene disruption strain. Cla4 also contributes to a similar style of response by delaying $\mathrm{G}_{2}-\mathrm{M}$ transition through prevention of proper bud formation, potentially through the septin ring formation, allowing the normal filamentation inputs to overdrive the filamentous response, leading to hyper-filamentation.

Studying the PAKs leads to two interesting ideas of signaling pathway overlap and specificity of the various signaling pathways leading to filamentation, and to the input of multiple pathways controlling morphogenesis and development. More work needs to be done to assign cell fate specific activity to both Cla4 and Smu1 under multiple conditions, as well as defining the multitude to potential targets and regulators of both PAKs. Exploring the PAK specific activity of the Rho/Rac family of GTPases 
would provide essential insight to the localization and activity of the two families of proteins in dimorphism and in cell fate. 


\section{REFERENCES}

Alexopoulos, C. J., Mims, C. W., and Blackwell, M. (1996). Introductory Mycology (4th Ed.). John Wiley and Sons, New York, USA. 868p.

Alvarez-Tabarés, I. \& Pérez-Martín, J. (2008). Cdk5 kinase regulates the association between adaptor protein Bem1 and GEF Cdc24 in the fungus Ustilago maydis. $J$. Cell Sci., 121, 2824-2832.

Asano, S., Park, J. E., Sakchaisri, K., Yu, L. R., Song, S., Supavilai, P. Veenstra, T. D., Lee, K. S. (2005). Concerted mechanism of Swe1/Wee1 regulation by multiple kinases in budding yeast. EMBO J., 24, 2194-2204.

Baek, S. T., Han, S., Nam, M., Kim, Y.D., Kim, L., Lee, H.J., Heo, K.S., Lee, H., Lee, M., Park, S.K., Maeng, P.J., Park, Y., Lee, S., Kim, D.U., Kim, D., \& Hoe, K.L. (2008). Genome-wide identification of haploinsufficiency in fission yeast. $J$. Microbiol. Biotechnol., 18, 1059-1063.

Banuett, F. (1995). Genetics of Ustilago maydis, a fungal pathogen that induces tumors in maize. Annu. Rev. Genetics, 29, 179-208.

Banuett, F., and Herskowitz, I. (1989). Different $a$ alleles of Ustilago maydis are necessary for maintenance of filamentous growth but not for meiosis. Proc. Natl. Acad. Sci. USA, 86, 5878-5882.

Bao, S., Qyang, Y., Yang, P., Kim, H., Du, H., Bartholomeusz, G. Henkel, J., Pimental, R., Verde, F., Marcus, S. (2001). The highly conserved protein methyltransferase, 
Skbl, is a mediator of hyperosmotic stress response in the fission yeast Schizosaccharomyces pombe. J. Biol. Chem., 276, 14549-14552.

Bardwell, L. (2005). A walk-through of the yeast mating pheromone response pathway. Peptides, 26, 339-350.

Bartholomew, C. R. \& Hardy, C. F. J. (2009). p21-activated kinases Cla4 and Ste20 regulate vacuole inheritance in Saccharomyces cerevisiae. Eukaryot. Cell, 8, 560572.

Bassilana, M. \& Arkowitz, R. A. (2006). Rac1 and Cdc42 have different roles in Candida albicans development. Eukaryot. Cell, 5, 321-329.

Benton, B. K., Tinkelenberg, A., Gonzalez, I., \& Cross, F. R. (1997). Cla4, a Saccharomyces cerevisiae Cdc42-activated kinase involved in cytokinesis, is activated at mitosis. Mol. Cell Biol., 17, 5067-5076.

Bertani, G. (1951). Studies on lysogenesis. I. The mode of phage liberation by lysogenic Escherichia coli. J. Bacteriol., 62, 293-300.

Böhmer, C., Böhmer, M., Bölker, M., \& Sandrock, B. (2008). Cdc42 and the Ste20-like kinase Don3 act independently in triggering cytokinesis in Ustilago maydis. $J$. Cell Sci., 121, 143-148.

Bok, J. W. \& Keller, N. P. (2004). LaeA, a regulator of secondary metabolism in Aspergillus spp. Eukaryot. Cell, 3, 527-535.

Bölker, M. (2001). Ustilago maydis - a valuable model system for the study of fungal dimorphism and virulence. Microbiology, 147, 1395-1401.

Bowman, S. M. \& Free, S. J. (2006). The structure and synthesis of the fungal cell wall. BioEssays, 28, 799-808. 
Brachmann, A., König, J., Julius, C., \& Feldbrügge, M. (2004). A reverse genetic approach for generating gene replacement mutants in Ustilago maydis. Mol. Genet. Genomics, 272, 216-226.

Brachmann, A., Weinzierl, G., Kämper, J., \& Kahmann, R. (2001). Identification of genes in the $\mathrm{bW} / \mathrm{bE}$ regulatory cascade in Ustilago maydis. Mol. Microbiol., 42, 1047-1063.

Cabib, E., Drgonová, J., \& Drgon, T. (1998). Role of small G proteins in yeast cell polarization and wall biosynthesis. Annu. Rev. Biochem., 67, 307-333.

Castillo-Lluva, S., Alvarez-Tabarés, I., Weber, I., Steinberg, G., \& Pérez-Martín, J. (2007). Sustained cell polarity and virulence in the phytopathogenic fungus Ustilago maydis depends on an essential cyclin-dependent kinase from the Cdk5/Pho85 family. J. Cell Sci., 120, 1584-1595.

Chang, E., Bartholomeusz, G., Pimental, R., Chen, J., Lai, H., Wang, L. Yang, P., Marcus, S. (1999). Direct binding and In vivo regulation of the fission yeast p21activated kinase Shk1 by the SH3 domain protein Scd2. Mol. Cell Biol., 19, 80668074.

Chen, R. E. \& Thorner, J. (2007). Function and regulation in MAPK signaling pathways: lessons learned from the yeast Saccharomyces cerevisiae. Biochim. Biophys. Acta., 1773, 1311-1340.

Cid, V. J., Shulewitz, M. J., McDonald, K. L., \& Thorner, J. (2001). Dynamic localization of the Swe1 regulator Hsl7 during the Saccharomyces cerevisiae cell cycle. Mol. Biol. Cell, 12, 1645-1669. 
Coll, P. M., Trillo, Y., Ametzazurra, A., \& Pérez, P. (2003). Geflp, a new guanine nucleotide exchange factor for Cdc42, regulates polarity in Schizosaccharomyces pombe. Mol. Biol. Cell, 14, 313-323.

Cvrčková, F., De, V. C., Manser, E., Pringle, J. R., \& Nasmyth, K. (1995). Ste20-like protein kinases are required for normal localization of cell growth and for cytokinesis in budding yeast. Genes Dev., 9, 1817-1830.

Deutschbauer, A.M., Jaramillo, D.F., Proctor, M., Kumm, J., Hillenmeyer, M.E., Davis, R.W., Nislow, C., \& Giaever, G. (2005). Mechanisms of haploinsufficiency revealed by genome-wide profiling in yeast. Genetics, 169, 1915-1925.

Doehlemann, G., van der Linde, K., Aßmann, D., Schwammbach, D., Hof, A., Mohanty, A. Jackson, D., Kahmann, R. (2009). Pep1, a secreted effector protein of Ustilago maydis, is required for successful invasion of plant cells. PLoS. Pathog., 5, e1000290.

Dürrenberger, F., Wong, K., \& Kronstad, J. W. (1998). Identification of a cAMPdependent protein kinase catalytic subunit required for virulence and morhpogenesis in Ustilago maydis. Proc. Natl. Acad. Sci. USA, 95, 5684- 5689.

Eby, J. J., Holly, S. P., van, D. F., Grishin, A. V., Peter, M., Drubin, D. G. Blumer, K. J. (1998). Actin cytoskeleton organization regulated by the PAK family of protein kinases. Curr. Biol., 8, 967-970.

Etienne-Manneville, S. (2004). Cdc42--the centre of polarity. J. Cell Sci., 117, 12911300.

Fedler, M., Luh, K., Stelter, K., Nieto-Jacobo, F., \& Basse, C. W. (2009). The a2 mating-type locus genes lga2 and rga2 direct uniparental mitochondrial DNA 
(mtDNA) inheritance and constrain mtDNA recombination during sexual development of Ustilago maydis. Genetics, 181, 847-860.

Feldbrügge, M., Kämper, J., Steinberg, G., \& Kahmann, R. (2004). Regulation of mating and pathogenic development in Ustilago maydis. Curr. Opin. Microbiol., 7, 666672.

Fujita, A., Tonouchi, A., Hiroko, T., Inose, F., Nagashima, T., Satoh, R. Tanaka, S. (1999). Hsl7, a negative regulator of Ste20 protein kinase in the Saccharomyces cerevisiae filamentous growth-signaling pathway. Proc. Natl. Acad. Sci. U.S.A, $96,8522-8527$.

Gagiano, M., Bauer, F. F., \& Pretorious, I. S. (2002). The sensing of nutritional status and the relationship to filamentous growth in Saccharomyces cerevisiae. FEMS Yeast Res., 2, 433-470.

García-Pedrajas, M. D., Nadal, M., Bölker, M., Gold, S. E., \& Perlin, M. H. (2008). Sending mixed signals: redundancy vs. uniqueness of signaling components in the plant pathogen, Ustilago maydis. Fungal. Genet. Biol., 45 Suppl 1, S22-S30.

Gilbreth, M., Yang, P., Bartholomeusz, G., Pimental, R. A., Kansra, S., Gadiraju, R. Marcus, S. (1998). Negative regulation of mitosis in fission yeast by the Shk1 interacting protein Skb1 and its human homolog, Skb1Hs. Proc. Natl. Acad. Sci. U.S.A, 95, 14781-14786.

Gilbreth, M., Yang, P., Wang, D., Frost, J., Polverino, A., Cobb, M. H. Marcus, S. (1996). The highly conserved Skbl gene encodes a protein that interacts with Shk1, a fission yeast Ste20/PAK homolog. Proc. Natl. Acad. Sci. U.S.A, 93, $13802-13807$ 
Gold, S. E., Brogdon, S. M., Mayorga, M. E., \& Kronstad, J. W. (1997). The Ustilago maydis regulatory subunit of a cAMP-dependent protein kinase is required for gall formation in maize. Plant Cell, 9, 1585-1594.

Gold, S. E., Duncan, G., Barrett, K., \& Kronstad, J. W. (1994). cAMP regulates morphogenesis in the fungal pathogen Ustilago maydis. Genes Dev., 8, 28052816.

Gulli, M. P. \& Peter, M. (2001). Temporal and spatial regulation of Rho-type guaninenucleotide exchange factors: the yeast perspective. Genes Dev., 15, 365-379.

Hawksworth, D. L. (1991). The fungal dimension of biodiversity: magnitude, significance, and conservation. Mycological Research, 95:641-655.

Heinrich, M., Köhler, T., \& Mösch, H. U. (2007). Role of Cdc42-Cla4 interaction in the pheromone response of Saccharomyces cerevisiae. Eukaryot. Cell, 6, 317-327.

Hlubek, A., Schink, K. O., Mahlert, M., Sandrock, B., \& Bölker, M. (2008). Selective activation by the guanine nucleotide exchange factor Don1 is a main determinant of Cdc42 signalling specificity in Ustilago maydis. Mol. Microbiol., 68, 615-623.

Höfken, T. \& Schiebel, E. (2002). A role for cell polarity proteins in mitotic exit. EMBO J., $21,4851-4862$.

Hogan, D. A. (2006). Talking to themselves: autoregulation and quorum sensing in fungi. Eukaryot. Cell, 5, 613-619.

Holliday, R. (1974). Ustilago maydis, Handbook of Genetics. (R. C. King Ed.) Plenum, New York, USA. 575-595p. 
Holly, S. P. \& Blumer, K. J. (1999). PAK-family kinases regulate cell and actin polarization throughout the cell cycle of Saccharomyces cerevisiae. J. Cell Biol., $147,845-856$.

International Potato Center. [http://cipotato.org]

Jacobs, C. W., Mattichak, S. J., \& Knowles, J. F. (1994). Budding patterns during the cell cycle of the maize smut pathogen Ustilago maydis. Can. J. Bot., 72, 16751680.

Kadota, J., Yamamoto, T., Yoshiuchi, S., Bi, E., \& Tanaka, K. (2004). Septin ring assembly requires concerted action of polarisome components, a PAK kinase Cla4, and the actin cytoskeleton in Saccharomyces cerevisiae. Mol. Biol. Cell, 15, $5329-5345$.

Kaffarnik, F., Müller, P., Leibundgut, M., Kahmann, R., \& Feldbrügge, M. (2003). PKA and MAPK phosphorylation of Prf1 allows promoter discrimination in Ustilago maydis. EMBO J., 22, 5817-5826.

Kahmann, R. \& Kämper, J. (2004). Ustilago maydis: how its biology relates to pathogenic development. New Phytologist, 164, 31-42.

Kämper, J. (2004). A PCR-based system for highly efficient generation of gene replacement mutants in Ustilago maydis. Mol. Genet. Genomics, 271, 103-110.

Kämper, J., Reichmann, M., Romeis, T., Bölker, M., \& Kahmann, R. (1995). Multiallelic recognition: nonself-dependent dimerization of the $\mathrm{bE}$ and $\mathrm{bW}$ homeodomain proteins in Ustilago maydis. Cell, 81, 73-83. 
Keniry, M. E. \& Sprague, G. F., Jr. (2003). Identification of p21-activated kinase specificity determinants in budding yeast: a single amino acid substitution imparts Ste20 specificity to Cla4. Mol. Cell Biol., 23, 1569-1580.

Klose, J., de Sá, M. M., \& Kronstad, J. W. (2004). Lipid-induced filamentous growth in Ustilago maydis. Mol. Microbiol., 52, 823-835.

Klosterman, S. J., Perlin, M. H., García-Pedrajas, M. D., Covert, S. F., \& Gold, S. E. (2007). Genetics of morphogenesis and pathogenic development of Ustilago maydis. Adv. Genet., 57, 1-47.

Kronstad, J. W. \& Leong, S. A. (1989). Isolation of two alleles of the b locus of Ustilago maydis. Proc. Natl. Acad. Sci. U.S.A, 86, 978-982.

La Valle, V. R. \& Wittenberg, C. (2001). A role for the Swel checkpoint kinase during filamentous growth of Saccharomyces cerevisiae. Genetics, 158, 549-562.

Lamson, R. E., Winters, M. J., \& Pryciak, P. M. (2002). Cdc42 regulation of kinase activity and signaling by the yeast p21-activated kinase Ste20. Mol. Cell Biol., 22, 2939-2951.

Leberer, E., Thomas, D. Y., \& Whiteway, M. (1997). Pheromone signaling and polarized morphogenesis in yeast. Curr. Opin. Genet. Dev., 7, 59-66.

Lee. J., Cook, J. R., Pollack, B. P., Kinzy, T. G., Norris, D., \& Pestka, S. (2000). Hsl7, the yeast homologue of human JBP1, is a protein methyltransferase. Biochem. Biophys. Res. Commun., 274, 105-111.

Lengeler, K. B., Davidson, R. C., D'souza, C., Harashima, T., Shen, W. C., Wang, P. Pan, X., Waugh, M., Heitman, J. (2000). Signal transduction cascades regulating fungal development and virulence. Microbiol. Mol. Biol. Rev., 64, 746-785. 
Leveleki, L., Mahlert, M., Sandrock, B., \& Bölker, M. (2004). The PAK family kinase Cla4 is required for budding and morphogenesis in Ustilago maydis. Mol. Microbiol., 54, 396-406.

Levin, A. M., de Vries, R. P., Conesa, A., de, B. C., Talon, M., Menke, H. H. van Peij, N.N., Wösten, H. A. (2007). Spatial differentiation in the vegetative mycelium of Aspergillus niger. Eukaryot. Cell, 6, 2311-2322.

Lyons, D. M., Mahanty, S. K., Choi, K., Manandhar, M., \& Eloin, E. A. (1996). The SH3-domain protein Bem1 coordinates mitogen-activated protein kinase cascade activation with cell cycle control in Saccharomyces cerevisiae. Mol. Cell Biol., 16, 4095-4106.

Ma, X. J. (2000). Cell-cycle regulatory proteins Hsl7/Skb1p belong to the protein methyltransferase superfamily. Trends Biochem. Sci., 25, 11-12.

Ma, X. J., Lu, Q., \& Grunstein, M. (1996). A search for proteins that interact genetically with histone $\mathrm{H} 3$ and $\mathrm{H} 4$ amino termini uncovers novel regulators of the Swe1 kinase in Saccharomyces cerevisiae. Genes Dev., 10, 1327-1340.

Madden, K. \&Snyder, M. (1998). Cell polarity and morphogenesis in budding yeast. Annu. Rev. Microbiol., 52, 687-744.

Madhani, H. D. \& Fink, G. R. (1998). The control of filamentous differentiation and virulence in fungi. Trends Cell Biol., 8, 348-353.

Mahlert, M., Leveleki, L., Hlubek, A., Sandrock, B., \& Bölker, M. (2006). Rac1 and Cdc42 regulate hyphal growth and cytokinesis in the dimorphic fungus Ustilago maydis. Mol. Microbiol., 59, 567-578. 
Marcus, S., Polverino, A., Chang, E., Robbins, D., Cobb, M. H., \& Wigler, M. H. (1995). Shk1, a homolog of the Saccharomyces cerevisiae Ste20 and mammalian p65PAK protein kinases, is a component of a Ras/Cdc42 signaling module in the fission yeast Schizosaccharomyces pombe. Proc. Natl. Acad. Sci. U.S.A, 92, 61806184.

Miranda, T. B., Sayegh, J., Frankel, A., Katz, J. E., Miranda, M., \& Clarke, S. (2006). Yeast Hsl7 (histone synthetic lethal 7) catalyzes the in vitro formation of $\omega-N^{G}-$ monomethylarginine in calf thymus histone H2A. Biochem. J., 395, 563-570.

Money, N. P. (2006). The triumph of the fungi: a rotten history. Oxford University Press Inc. New York, USA. 102-111p.

Nadal, M., García-Pedrajas, M. D., \& Gold, S. E. (2008). Dimorphism in fungal plant pathogens. FEMS Microbiol. Lett., 284,127-34.

Nakano, K., Mutoh, T., Arai, R., \& Mabuchi, I. (1997). The small GTP-binding protein Rhol is a multifunctional protein that regulates actin localization, cell polarity, and septum formation in the fission yeast Schizosaccharomyces pombe. Genes Cells, 2, 679-694.

Neiman, A. M., Stevenson, B. J., Xu, H. P., Sprague, G. F., Jr., Herskowitz, I., Wigler, M. Marcus, S. (1993). Functional homology of protein kinases required for sexual differentiation in Schizosaccharomyces pombe and Saccharomyces cerevisiae suggests a conserved signal transduction module in eukaryotic organisms. Mol. Biol. Cell, 4, 107-120. 
Nelson, W. J. (2003). Mum, this bud's for you: where do you want it? Roles for Cdc42 in controlling bud site selection in Saccharomyces cerevisiae. BioEssays, 25, 833836.

Nichols, C. B., Fraser, J. A., \& Heitman, J. (2009). PAK kinases Ste20 and Pak1 govern cell polarity at different stages of mating in Cryptococcus neoformans. Eukaryot. Cell, 15, 4476-4489.

Oehlen, L. J. W. M. \& Cross, F. R. (1998). Potential regulation of Ste20 function by the $\mathrm{Cln} 1-\mathrm{Cdc} 28$ and $\mathrm{Cln} 2-\mathrm{Cdc} 28$ cyclin-dependent protein kinases. J. Biol. Chem., 273, 25089-25097.

Orlowski, M. (1991). Mucor dimorphism. Microbiol. Rev., 55, 234-258.

Pal, S., Vishwanath, S. N., Erdjument-Bromage, H., Tempst, P., Sif, S. (2004). Human SWI / SNF-associated PRMT5 methylates histone H3 arginine 8 and negatively regulates expression of ST7 and NM23 tumor suppressor genes. Mol. Cell Biol., $24,9630-9645$.

Palecek, S. P., Parikh, A. S., \& Kron, S. J. (2000). Genetic analysis reveals that FLO11 upregulation and cell polarization independently regulate invasive growth in Saccharomyces cerevisiae. Genetics, 156, 1005-1023.

Pham, C. D., Yu, Z., Sandrock, B., Bölker, M., Gold, S. E., \& Perlin, M. H. (2009). Ustilago maydis Rhol and 14-3-3 homologues participate in pathways controlling cell separation and cell polarity. Eukaryot. Cell, 8, 977-989.

Plant and Fungi, Ustilago maydis. [http://www.kew.org/plants-fungi/Ustilagomaydis.htm] 
Pollack, B. P., Kotenko, S. V., He, W., Izotova, L. S., Barnoski, B. L., \& Pestka, S. (1999). The human homologue of the yeast proteins Skb1 and Hs17 interacts with Jak kinases and contains protein methyltransferase activity. J. Biol. Chem., 274, 31531-31542.

Qyang, Y., Yang, P., Du, H., Lai, H., Kim, H., \& Marcus, S. (2002). The p21-activated kinase, Shk 1 , is required for proper regulation of microtubule dynamics in the fission yeast, Schizosaccharomyces pombe. Mol. Microbiol., 44, 325-334.

Rho, J., Choi, S., Seong, Y. R., Cho, W., Kim, S. H., \& Im, D. (2001). PRMT5, which forms distinct homo-oligomers, is a member of the protein-arginine methyltransferase family. J. Biol. Chem., 276, 11393-11401.

Roberts, R. L. \& Fink, G. R. (1994). Elements of a single MAP kinase cascade in Saccharomyces cerevisiae mediate two developmental programs in the same cell type: mating and invasive growth. Genes Dev., 8, 2974-2985.

Roberts, R. L., Mösch, H. U., \& Fink, G. R. (1997). 14-3-3 proteins are essential for RAS / MAPK cascade signaling during pseudohyphal development in S. cerevisiae. Cell, 89, 1055-1065.

Ruiz-Herrera, J., León, C. G., Guevara-Olvera, L., \& Cárabez-Trejo, A. (1995). Yeastmycelial dimorphism of haploid and diploid strains of Ustilago maydis. Microbiology, 141, 695-703.

Sánchez-Martínez, C. \& Pérez-Martín, J. (2001). Dimorphism in fungal pathogens: Candida albicans and Ustilago maydis - similar inputs, different outputs. Curr. Opin. Microbiol., 4, 214-221. 
Schink, K. O. \& Bölker, M. (2009). Coordination of cytokinesis and cell separation by endosomal targeting of a Cdc42-specific guanine nucleotide exchange factor in Ustilago maydis. Mol. Biol. Cell, 20, 1081-1088.

Scoumanne, A., Zhang, J., \& Chen, X. (2009). PRMT5 is required for cell-cycle progression and p53 tumor suppressor function. Nucleic Acids Res., 37, 49654976.

Sgarlata, C. \& Pérez-Martín, J. (2005). Inhibitory phosphorylation of a mitotic cyclindependent kinase regulates the morphogenesis, cell size and virulence of the smut fungus Ustilago maydis. J. Cell Sci., 118, 3607-3622.

Shelton, 2001. [http://www.plant.uga.edu/labrat/ergot.htm]

Shulewitz, M. J., Inouye, C. J., \& Thorner, J. (1999). Hsl7 localizes to a septin ring and serves as an adapter in a regulatory pathway that relieves tyrosine phosphorylation of $\mathrm{Cdc} 28$ protein kinase in Saccharomyces cerevisiae. Mol. Cell Biol., 19, 7123-7137.

Sipiczki, M. (2007). Splitting of the fission yeast septum. FEMS Yeast Res., 7, 761-770. Smith, D. G., Garcia-Pedrajas, M. D., Gold, S. E., \& Perlin, M. H. (2003). Isolation and characterization from pathogenic fungi of genes encoding ammonium permeases and their roles in dimorphism. Mol. Microbiol., 50, 259-275.

Smith, D. G., Garcia-Pedrajas, M. D., Hong, W., Yu, Z., Gold, S. E., \& Perlin, M. H. (2004). An Ste20 homologue in Ustilago maydis plays a role in mating and pathogenicity. Eukaryot. Cell, 3, 180-189.

Smits, G. J., van den Ende, H., \& Klis, F. M. (2006). Differential regulation of cell wall biogenesis during growth and development in yeast. Microbiology, 147, 781-794. 
Specialty Produce. [http://www.SpecialtyProduce.com]

Steinberg, G., Schliwa, M., Lehmler, C., Bölker, M., Kahmann, R., \& McIntosh, J. R. (1998). Kinesin from the plant pathogenic fungus Ustilago maydis is involved in vacuole formation and cytoplasmic migration. J. Cell Sci., 111 (Pt 15), 22352246.

Takahashi, S. \& Pryciak, P. M. (2007). Identification of novel membrane-binding domains in multiple yeast Cdc42 effectors. Mol. Biol. Cell, 18, 4945-4956.

Tapon, N. \& Hall, A. (1997). Rho, Rac and Cdc42 GTPases regulate the organization of the actin cytoskeleton. Curr. Opin. Cell Biol., 9, 86-92.

Tolliday, N., VerPlank, L., \& Li, R. (2002). Rho1 directs formin-mediated actin ring assembly during budding yeast cytokinesis. Curr. Biol., 12, 1864-1870.

Uhl, M. A., Biery, M., Craig, N., \& Johnson, A.D. (2003). Haploinsufficiency-based large-scale forward genetic analysis of filamentous growth in the diploid human fungal pathogen C. albicans. EMBO J., 22, 2668-2678.

USDA, 2009. [http://www.ers.usda.gov/briefing/corn/]

Veitia, R. A. (2002). Exploring the etiology of haploinsufficiency. BioEssays, 24, 175184.

Versele, M. \& Thorner, J. (2004). Septin collar formation in budding yeast requires GTP binding and direct phosphorylation by the PAK, Cla4. J. Cell Biol., 164, 701-715.

Versele, M. \& Thorner, J. (2005). Some assembly required: yeast septins provide the instruction manual. Trends Cell Biol., 15, 414-424.

Wang, P., Nichols, C., Lengeler, K. B., Cardenas, M. E., Cox, G. M. Perfect, J. R., \& Heitman, J. (2002). Mating-type-specific and nonspecific PAK kinases play 
shared and divergent roles in Cryptococcus neoformans. Eukaryot. Cell, 1, 257272.

Weber, I., A $\beta$ mann, D., Thines, E., \& Steinberg, G. (2006). Polar localizing class V myosin chitin snythases are essential during early plant infection in the plant pathogenic fungus Ustilago maydis. Plant Cell, 18, 225-242.

Weinzierl, G., Leveleki, L., Hassel, A., Kost, G., Wanner, G., \& Bölker, M. (2002). Regulation of cell separation in the dimorphic fungus Ustilago maydis. Mol. Microbiol., 45, 219-231.

Wild, A. C., Yu, J. W., Lemmon, M. A., \& Blumer, K. J. (2004). The p21-activated protein kinase-related kinase $\mathrm{Cla} 4$ is a coincidence detector of signaling by $\mathrm{Cdc} 42$ and phosphatidylinositol 4-phosphate. J. Biol. Chem., 279, 17101-17110.

Wiley, D. J., Marcus, S., D'urso, G., \& Verde, F. (2003). Control of cell polarity in fission yeast by association of Orb6 p kinase with the highly conserved protein methyltransferase Skblp. J. Biol. Chem., 278, 25256-25263.

Winters, M. J. and Pryciak,P. M. (2005). Interaction with the SH3 domain protein Bem1 regulates signaling by the Saccharomyces cerevisiae p21-activated kinase Ste20. Mol. Cell Biol., 25, 2177-2190.

Wu, C., Leeuw, T., Leberer, E., Thomas, D. Y., \& Whiteway, M. (1998). Cell cycleand $\mathrm{Cln} 2 \mathrm{p}$-Cdc28-dependent phosphorylation of the yeast Ste20 protein kinase. J. Biol. Chem., 273, 28107-28115.

Yamada, A., Duffy, B., Perry, J. A., \& Kornbluth, S. (2004). DNA replication checkpoint control of Weel stability by vertebrate Hs17. J. Cell Biol., 167, 841-849. 
Yang, P., Kansra, S., Pimental, R. A., Gilbreth, M., \& Marcus, S. (1998). Cloning and characterization of shk2, a gene encoding a novel p21-activated protein kinase from fission yeast. J. Biol. Chem., 273, 18481-18489.

Ziman, M., Preuss, D., Mulholland, J., O'Brien, J. M., Botstein, D., \& Johnson, D. I. (1993). Subcellular localization of Cdc42, a Saccharomyces cerevisiae GTPbinding protein involved in the control of cell polarity. Mol. Biol. Cell, 4, 13071316 


\section{APPENDIX I \\ HAPLO-INSUFFICIENCY IN THE HSL7 MUTANT BACKGROUND INTRODUCTION AND DISSCUSSION}

Diploid organisms exhibit allelic compensation for most genes. This is defined as a loss of function allele with no discernable phenotype. The one functional allele masks the phenotypic consequences of the loss of the function of the second allele. Ninety seven percent of $S$. cerevisiae genes display allelic compensation (Duetschbaur et al., 2005). However, in up to three percent of genes, loss of function in one allele can lead to harmful effects on the diploid cells and such deviations from wild type phenotypes are termed as haplo-insufficiency (H-I). More commonly observed in multi-cellular organisms, $\mathrm{H}-\mathrm{I}$ has been shown to cause many problems in humans in chromosome maintenance and gene expression. The transcription factors TWIST and GATA3, as well as the DNA helicase BLM and DNA repair proteins (ATM) all exhibit H-I defects (Duetschbaur et al., 2005). Examples such as these have lead to many diseases such as Marfan syndrome, cancer, and Diamond Blackfan Anemia (DBA) (Baek, et al., 2008; Dr. S Ellis, personal communication).

Though observed in $S$. cerevisiae, $S$. pombe, and C. albicans, H-I has not been extensively studied in fungi and only recently has $\mathrm{H}-\mathrm{I}$ been examined in the maize pathogen Ustilago maydis (Dr. C. Pham, PhD dissertation). The two highly conserved genes, the Rho-like GTPase, rhol, and the 14-3-3e homologue, $p d c 1$, have demonstrated 
H-I defects. Rhol and Pdc1 are required for maintenance of polarity and cytokinesis in U. maydis (Pham, et al., 2009). In addition, diploids lacking one allele of rhol (rho1:Arhol) were avirulent as well as unable to produce or detect pheromone, form vacuoles, and produce hyphae. Single copy $p d c 1$ diploid strains $(p d c 1: \Delta p d c 1)$ were able to infect plants normally when the method of plant inoculation was stem injection, however when dropped onto the whorl of the plant, a decrease in tumor formation was observed (Dr. C. Pham, personal communication).

In $U$. maydis, mating and pathogenic development are regulated by two highly conserved pathways, the cAMP-dependent PKA pathway and the MAPK pathway (for review, see Klosterman, et al., 2007). Both pathways relay extracellular signals to the nucleus and trigger differential gene expression. One target of the two pathways is the pheromone responsive transcription factor, Prf1, which regulates expression of both the $a$ and $b$ mating loci. The two pathways also are involved in filamentous response to environmental conditions, i.e., low ammonium (SLAD) and acid $\mathrm{pH}$. The role of the MAPK pathway in regulating both the mating response and filamentous growth may be dependent on the p21-activated protein kinase (PAK) Smu1. Loss of smu1 attenuated the up-regulation of pheromone expression in the $a 2$ mating-type background in response to basal levels of pheromone from the al mating-type strain, resulting in a decrease in the overall mating response and, ultimately, virulence (Smith et al., 2004). It was also observed that Smul played a role in the filamentous response to low ammonium conditions, though the mechanism for this regulation is not understood (Smith, et al., 2004; Chapter II). In addition, mating-type specific perturbations in cell length were observed in smul mutant strains as previously described (Chapter II). 
Similar effects on the cell length and the filamentous response to low ammonium conditions were observed in strains either lacking or overexpressing hsl7 (Chapter II). Hsl7 is a putative protein-arginine methyltransferase that plays a role in cell cycle regulation in the G2-M transition in S. cerevisiae, S. pombe, and X. laevis (Gilbreth, et al., 1998; La Valle and Wittenberg, 2001; Yamada, et al., 2004; Asano, et al., 2005). In addition, it has been demonstrated that $\mathrm{Hsl} 7$ from $S$. cerevisiae regulates the filamentous response to environmental conditions, though the exact mechanism is disputed (Fujita, et al., 1999; La Valle and Wittenberg, 2001). One mechanism of filamentous regulation has Hs17 interacting with Ste20 in a negative manner (Fujita, et al., 1999). Analogous interactions between Hsl7 homologues and Ste20 homologues have been observed in both S. pombe and human (Gilbreth, et al., 1996 and 1998; Bao, et al., 2001; Pollack, et al., 1999). In U. maydis, genetic interaction studies previously performed (Chapter II) indicated that the interaction between Smul and Hsl7 may not be a direct interaction as observed in S. cerevisiae. However, concomitant disruption of $h s l 7$ and overexpression of $s m u l$ leads to hyper-filamentation on SLAD and displays mating-type specific defects in cell separation.

The mating-type defects exhibited by smul and $h s l 7$ mutant strains lead to the exploration of H-I in these strains. To explore H-I in these genes, strains with a single gene replacement with a carboxin drug resistance cassette of either $s m u l$ or $h s l 7$ were created in the diploid strain D132. Examination of cell morphology of both the hsl7:Ahsl7 and smul:Asmul diploid cells indicated no difference between either mutant strain and the wild type strain (data not shown). In addition, examination of colony morphology of both the $h s l 7: \Delta h s l 7$ and the smul:Asmul diploid strains on rich (data not 
shown) and low ammonium media indicated no difference when compared to the wild type strain (Fig. 38). Next the pathogenicity of both mutant strains was examined. Two
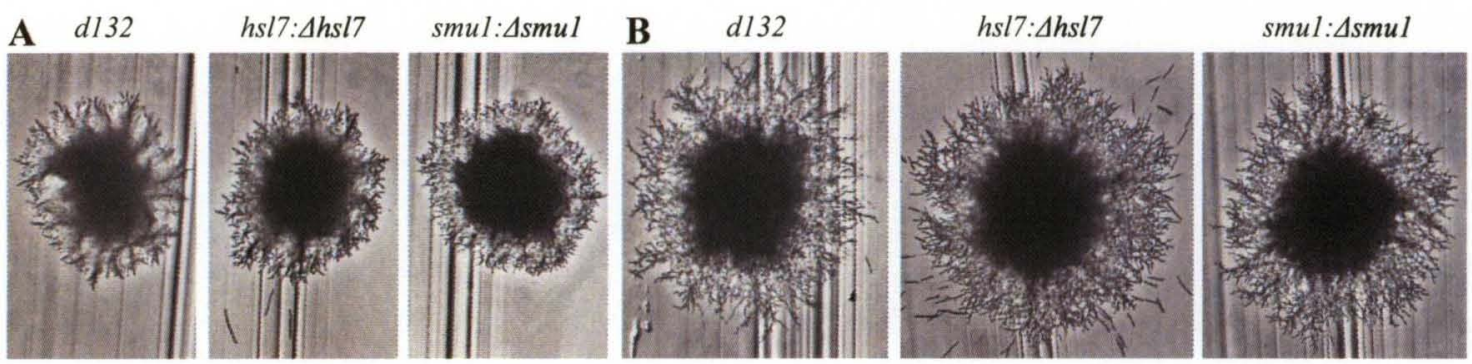

Figure 38: Filamentous response to low ammonium conditions of the diploid mutant strains. The strains were grown on SLAD media for 6 days and examined for filament formation at 3 days (A) and 6 days (B). Neither the $h s l 7: \Delta h s l 7$ nor the $s m u l: \Delta s m u l$ diploid strains differed from wild type in response to SLAD media.

different methods of inoculation were utilized, the first being injection of an inoculum of cells into the stem of the corn plant; in the second method, cells were dropped into the whorl of the plant. Measures of virulence were taken at 21 days post inoculation (dpi) and three independent trials of approximately 10 plants were performed for both injected (i) and whorl dropped (w) strains. The virulence for each infection was measured by a disease index, ranking the severity of the disease symptoms on a scale from 0 to 5 (as described in Chapter II of this dissertation) and the results of an ANOVA and a Tukey's post-hoc analysis are shown in Table 13. The results are also presented in a percent of symptom formation graph (Doehlemann, et al., 2009; see Fig. 39). The smul:Asmu1 diploid strain did not display any significant difference from the wild type strain in the ability to infect maize by either method of inoculation. However, the $h s l 7: \Delta h s l 7$ diploid strain exhibited a statistically significant decrease in virulence compared to the wild type strain when inoculated by injection. However, virulence of the $h s l 7: \Delta h s l 7$ diploid strain inoculated via whorl drop did not display any difference in pathogenicity compared with the wild type strain. Both the wild type and the smu1:Asmul diploid strains were able to 
TABLE 13. Pathogenicity of mutant strains ${ }^{A}$.

\begin{tabular}{|c|c|c|c|c|c|c|c|c|c|c|}
\hline \multirow{2}{*}{\multicolumn{2}{|c|}{ Treatment $^{\mathrm{B}}$}} & \multirow[t]{2}{*}{ Strain } & \multirow{2}{*}{$\begin{array}{l}\text { Plants } \\
\text { Tested }\end{array}$} & \multicolumn{6}{|c|}{ Disease rating ${ }^{\mathrm{C}}$ by number of affected plants } & \multirow{2}{*}{$\begin{array}{l}\text { Disease } \\
\text { Index }\end{array}$} \\
\hline & & & & $\begin{array}{c}0 \\
\text { Points }\end{array}$ & $\begin{array}{c}1 \\
\text { Point }\end{array}$ & $\begin{array}{c}2 \\
\text { Points }\end{array}$ & $\begin{array}{c}3 \\
\text { Points }\end{array}$ & $\begin{array}{c}4 \\
\text { Points } \\
\end{array}$ & $\begin{array}{c}5 \\
\text { Points } \\
\end{array}$ & \\
\hline \multirow[t]{3}{*}{ A } & 1 & D132 & 30 & 0 & 10 & 12 & 4 & 4 & 0 & 2.07 \\
\hline & 2 & DI32Ahsl7 & 30 & 3 & 24 & 3 & 0 & 0 & 0 & $1.00^{* *}$ \\
\hline & 3 & D1324smul & 29 & 2 & 9 & 14 & 2 & 2 & 0 & 1.76 \\
\hline \multirow[t]{3}{*}{$\mathrm{B}$} & 1 & D132 & 30 & 17 & 12 & 1 & 0 & 0 & 0 & 0.47 \\
\hline & 2 & D1324hsl7 & 30 & 17 & 13 & 0 & 0 & 0 & 0 & 0.43 \\
\hline & 3 & D1324smul & 30 & 20 & 10 & 0 & 0 & 0 & 0 & 0.33 \\
\hline
\end{tabular}

A Table shows combined data from three identical experiments of 9 to 10 plants each reported for 21 days postinoculation.

${ }^{B}$ Treatment consisted of the inoculation of $10^{7}$ cells $\mathrm{ml}^{-1}$ for each of the strains. Treatment A plants were inoculated by injection. Treatment B plants were inoculated via the fungal strain dropped into the whorl of the plant.

${ }^{\mathrm{C}}$ The disease rating is measured on a scale of 0 to 5 points based on the severity of symptoms as follows: 0 points, no disease symptoms; 1 point, chlorosis/anthocyanin production; 2 points, small leaf galls; 3 points, small galls on stems; 4 points, large stem galls; 5 points, plant death.

D The disease index is calculated as the sum of the disease ratings divided by the number of plants tested and statistical analysis was performed using a Kruskal-Wallis ANOVA with a Dunn's Multiple Comparison Test from three independent experiments.

** $p$ value less than .01 .

produce many small and several large galls, whereas the $h s l 7: \Delta h s l 7$ diploid strain only produced a few extremely small galls. A potential explanation for this difference could be that cells inoculated by injection are introduced to plant material thatis not as hardy as surface tissue, which is more lignified and harder to penetrate. Thus infections set up by injection may demonstrate defects of the $h s l 7: \Delta h s l 7$ diploid strain compared to the wild type strain, whereas the difficulty of plant penetration when strains are introduced by whorl drop conceals any difference in virulence between the mutant and wild type strain. 


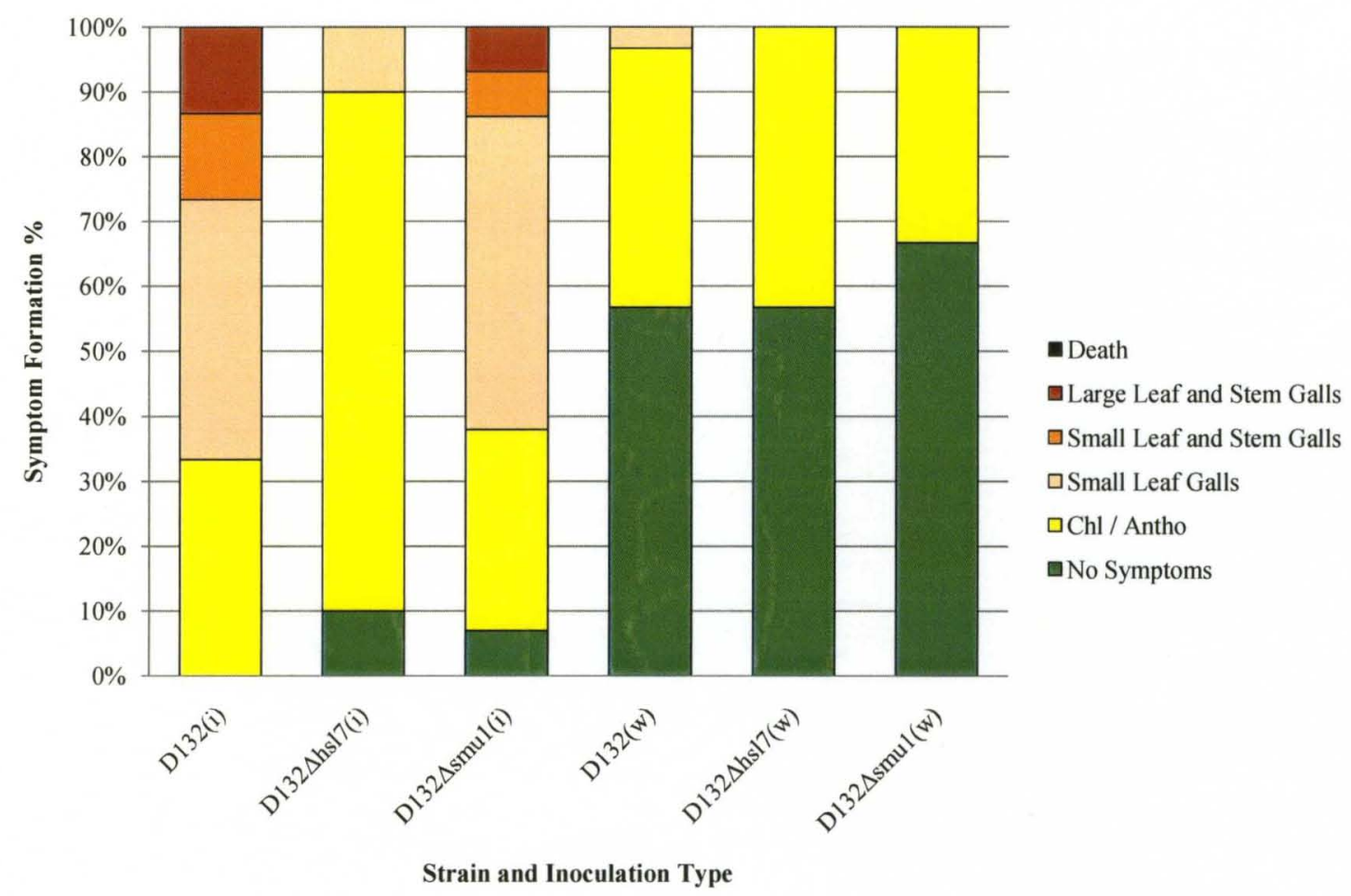

Figure 39: Disease symptom formation in the H-I $\boldsymbol{U}$. maydis mutant strains. All strains were rated at $21 \mathrm{dpi}$ and represent three independent experiments. Plants were inoculated with by one of two methods, injections in the plant stem (i) or dropped in the whorl of the plant (w). The graph displays the percentage of plants with a specific symptom of infections. The $h s l 7: \Delta h s l 7$ diploid strain failed to produce large galls as observed in both the wild type and smul: $\Delta s m u 1$ diploid strains, but only when inoculated by injection. No difference was observed from either mutant strain when the plants were inoculated by the whorl drop.

$\mathrm{H}-\mathrm{I}$ in fungi has only been reported in a handful of species, though it is a growing area of research. Work in S.cerevisiae has yielded evidence that up to $3 \%$ of genes display H-I, while the number of genes in $S$. pombe is 22, and 146 genes in C. albicans exhibit H-I (Uhl, et al., 2003; Deutschbauer, et al., 2005; Baek, et al., 2008). Work in $U$. maydis has now implicated 3 genes, rhol, $p d c 1$, and $h s l 7$ that show evidence of H-I (This work and Dr. C. Pham, PhD dissertation). H-I phenotypes may be due to one of two defects in expression. In H-I genes, there may be a dosage defect, where the cells require a set minimum amount of protein to promote a specific cell activity and by deleting one of the two alleles of the gene there may be a decrease in overall protein levels. An alternate hypothesis is that a stoichiometric perturbation creates an imbalance in protein 
ratios required in regulating a cellular process, thus leading to an H-I defect (Veitia, 2002). The $h s l 7: \Delta h s l 7$ diploid strain showed a marked reduction in gall formation when inoculated by injection, though no other defects were observed. The lack of an observable phenotype in the $h s l 7: \Delta h s l 7$ diploid strain when inoculated by whorl drop indicates a confounding phenotype that needs to be further explored.

\section{MATERIALS AND METHODS}

Ustilago maydis strains utilized in this study are listed in Table 14.

TABLE 14. $U$. maydis strains and plasmids used in this study.

\begin{tabular}{|c|c|c|}
\hline \multicolumn{3}{|c|}{ Fungal Strains ${ }^{\mathrm{A}}$} \\
\hline $\mathrm{d} 132$ & $a 1 a 2 b 1 b 2$ & Kronstad and Leong, 1989 \\
\hline d132 $\Delta h s l 7$ & 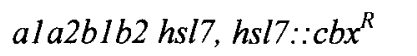 & This Study \\
\hline $\mathrm{d} 132 \Delta s m u I$ & $a 1 a 2 b 1 b 2$ smul, smul::cbx $x^{R}$ & This Study \\
\hline
\end{tabular}

${ }^{A}$ All mutant strains were made in the wild type background strains listed.

Primer design, PCR, vector construction and deletion construction, growth conditions, and statistical analysis, are described previously (Materials and methods, Chapter II). Plant infection using 8 day old Golden Bantam corn seedlings (Bunton Seed Co., Louisville, KY and W. Atlee Burpee \& Co., Warminster, PA) was performed with a cell density of $4 \times 10^{7}$ cells / $\mathrm{mL}$ for diploid strains as previously described (Gold et al., 1997). Plant inoculation was performed by either injecting strains into the corn stem or dropping strains into the whorl of the plant and symptoms were recorded for each plant 21 days post inoculation ( 29 days post planting) and the indices for each strain were averaged to give a measure per strain. Three independent trials of approximately 10 plants were performed, averaged, and analyzed. 


\section{APPENDIX II \\ MATING-TYPE SPECIFIC CELL WALL SENSITIVITY IN A STRAIN LACKING HSL7 \\ RESULTS AND DISCUSSION}

Fungal cells are surrounded by a thick cell wall comprised of three different components, $\sim 60 \% \beta$-glucans ( $\sim 50 \%$ 1,3- $\beta$-glucan; $\sim 10 \%$ 1,6- $\beta$-glucan $), \sim 40 \%$ mannoproteins, and $\sim 1 \%$ chitin (Smits, et al., 2001). Cross-linking between the various components of the cell wall creates rigidity in the cell wall and can limit cell growth and morphology. Ultimately, the cell wall needs to be a dynamic environment to allow for developmental morphogenesis. During the cell cycle of S. cerevisiae, cell wall plasticity allows for bud development and growth. During both mating filament formation and pseudohyphal development, a highly specific and organized apical form of growth occurs where cell wall material must be localized and deposited at the growing tip, thus promoting cell elongation. In addition, cytokinesis is dependent on deposition and subsequent degradation of a chitin rich septum separating mother and daughter cells (Smits, et al., 2001).

Chitin is a long homopolymer of $\beta$-1,4-linked $\mathrm{N}$-acetylglucosamine. As Nacetylglucosamine is excreted and polymerized into long chains, hydrogen bonding between the chains forms microfibrils and crystallizes the chitin (Bowman and Free, 2006). This process occurs at sites of active growth and cell wall remodeling, including 
the bud neck during cell separation and the hyphal tip and septa in filamentous fungi, is catalyzed by chitin synthases. In yeast, three chitin synthases (Chs) have been identified and are important, specifically for cell wall repair (Chslp), primary septum formation (Chs2p), and overall chitin synthesis (Chs3p). Deletion of all three chitin synthases is lethal, indicating chitin is an integral part of the fungal cell wall (Bowman and Free, 2006).

As described previously, a putative protein-arginine $\mathrm{N}$-methyltransferase homolog was identified in U. maydis (Chapter II). Hsl7 was demonstrated to interact with the Ste20 homologue, Smu1, in yeast two-hybrid analysis. Subsequent work indicated that Hsl7 plays a role in cell length, potentially by regulating the cell cycle, and the filamentous response to low ammonium conditions. As a means of comparison, two $\Delta h s l 7$ mutant strains of opposite mating-type (MUM11 alb1 and MUM12 a2b2) were obtained from Dr. José Pérez-Martín (Campus de Cantoblanco-UAM, Madrid, Spain). Examination of cellular morphology indicated that both $M U M 11$ and $M U M 12$ displayed statistically significant increases in cell length compared to the wild type strains, independent of mating-type background (Fig. 40A and Table 15). In addition, the MUM strains and the $\Delta_{810-2832} h s l 7$ strains did not differ in cell length, indicating similar effects on cell length from both $\Delta h s l 7$ mutant backgrounds. Examination of growth rates in the $M U M$ strains did not indicate any perturbations in cell cycle, thus growth was similar to that observed in the $\Delta_{810-2832} h s l 7$ background strains (Table 16). However, staining of the cell wall with CFW did indicate aberrations in mother daughter cell separation in both mating-type backgrounds. Both $M U M 11$ and $M U M 12$ strains exhibited groups of cells that failed to separate and produced cross walls, though not every cell displayed these 
TABLE 15. Measures of cell length of MUM11 and MUM12 strains.

\begin{tabular}{ccccc} 
Strain & $\mathrm{n}$ & Length $^{\mathrm{A}}$ (in $\left.\mu \mathrm{m}\right)$ & Comparison & $p^{B}$ \\
\hline WT albl & 91 & $19.27+/-.40$ & WT alb1 v. WT a2b2 & 0.82 \\
\cline { 4 - 5 } MUMIl albl & 70 & $25.11+/-.62$ & WT alb1 v. MUMII al & $>.001$ \\
$\Delta_{810-2832} h s l 7$ al & 167 & $25.22+/-.45$ & MUM11 alb1 v. $\Delta_{810-2832} h s l 7$ al & N.S. \\
\hline WT a2b2 & 213 & $19.14+/-.44$ & MUM1l a1 v. MUM12 a2 & 0.98 \\
\cline { 4 - 5 } MUMI2 a2b2 & 308 & $25.12+/-.44$ & WT a2b2 v. MUM12 a2 & $>.001$ \\
$\Delta_{810-2832} h s l 7$ a2 & 406 & $24.78+/-.32$ & MUM12 a2b2 v. $\Delta_{810-2832} h s l 7$ a2 & N.S. \\
\hline
\end{tabular}

${ }^{A}$ Cell length values are averages, +/- S.E.

B Statistical analysis was performed using a one way ANOVA with a Dunnett's Multiple Comparison Test and a Student's t-test.

TABLE 16. Growth rate analysis of mutant strains ${ }^{A}$.

\begin{tabular}{ccc} 
Strains & Double Time $^{\mathrm{B}}$ & $p^{C}$ \\
\hline WT alb1 & $2.76+/-.18$ & \\
MUM11 alb1 & $2.74+/-.19$ & 0.82 \\
\hline WT a2b2 & $2.77+/-.20$ & 0.95 \\
MUM12 a2b2 & $2.87+/-.11$ & 0.31 \\
\hline
\end{tabular}

A Table shows growth rate analysis of six identical experiments per strain.

${ }^{B}$ Growth rate expressed as doubling time in hours, +/- S.E.

c Statistical analysis was performed using a one way ANOVA with a Tukey's Multiple Comparison Test.

defects (Fig. 40B, see arrows). As a measure, the number of cross walls (cell walls that split a single cell in two, compared to mother daughter septa that divide two different cells) were counted per cluster (or group) of cells (Table 17). In $M U M 11$, nearly $48 \%$ cross wall formation was observed while no cross walls were observed in the wild type progenitor strain. Whereas in $M U M 12$, only $24 \%$

cross wall formation was observed, though the wild type $a 2 b 2$ mating-type strain produce $12 \%$ cross wall formation. Unlike cell morphology and growth rate analysis, cross wall 

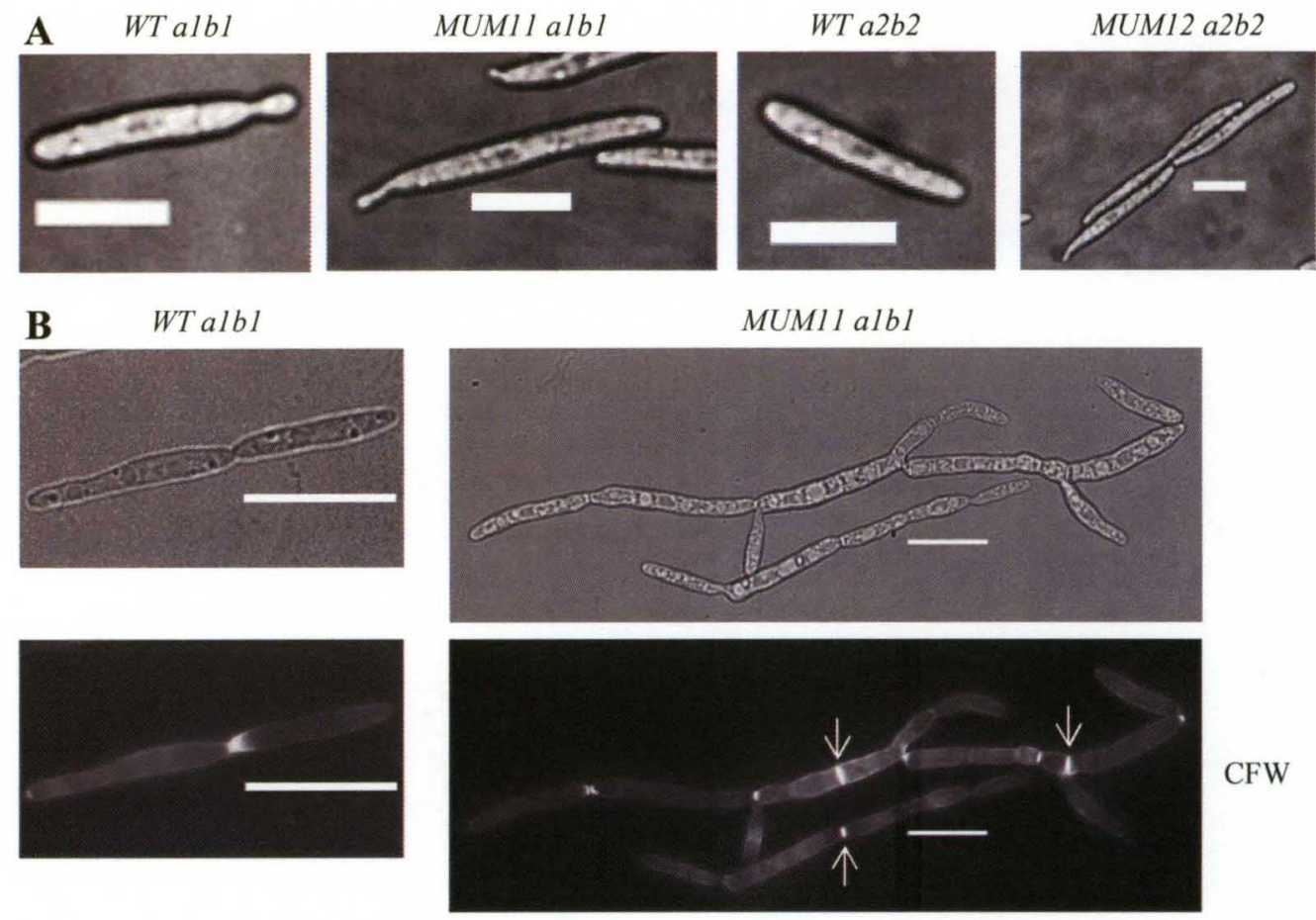

CFW
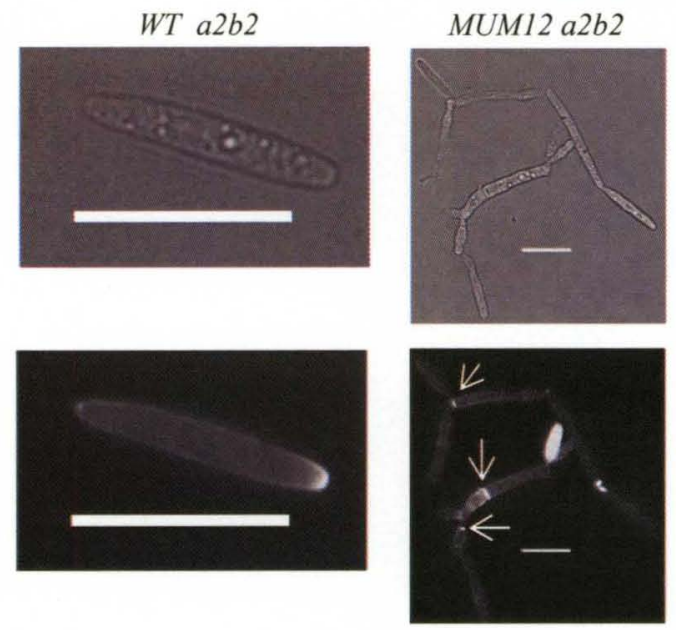

Figure 40: $M U M$ strains display an increase in cell length and cross wall septa. $M U M$ cells display an increase in cell length independent of mating-type background (A). Staining with CFW indicated $\mathrm{CFW}$ increase cross wall production in the $M U M$ strains (B, see arrows). Scale bars, $10 \mu \mathrm{m}$.

formation was only observed in the $M U M$ strains, not the $\Delta_{810-2832} h s l 7$ strains, and the degree of formation was mating-type specific.

Colony and micro-colony morphology was examined to determine if the production of cross walls would affect colony shape and structure. When grown on YEPS media, MUM11 and MUM12 displayed the small protrusions observed in the $\Delta_{810}$ ${ }_{2832} h s l 7$ mutant strains (Fig. 41A, see arrows). Investigation of micro-colony morphology 
TABLE 17. Cross wall formation.

\begin{tabular}{cccc} 
Strain & Cross walls $^{\mathrm{A}}$ & Clusters $^{\mathrm{B}}$ & Percentage \\
\hline WT albl & 0 & 90 & $0.0 \%$ \\
MUM11 alb1 & 76 & 159 & $47.8 \%$ \\
\hline WT a2b2 & 15 & 123 & $12.2 \%$ \\
MUM12 a2b2 & 58 & 241 & $24.0 \%$ \\
\hline${ }^{\mathrm{A}}$ Cross walls were counted as cell walls that split a single \\
cell in two, different than mother / daughter septa cell \\
division. \\
${ }^{\mathrm{B}}$ Clusters consist of either single cells or groups of cells \\
that were connected.
\end{tabular}

revealed the "directional growth" phenotype previously observed (Fig. 41; Chapter II). colonies (Fig. 42A and B). The increase in filament formation on SLAD raised the question of whether loss of $h s l 7$ affected filament formation during the mating reaction. During a plate mating assay, compatible haploid cells of opposite mating-type background were mixed and spotted on YPD media containing activated charcoal. A positive mating reaction produces aerial hyphae that appear white on the black background. No defects in the mating response were observed in the $M U M$ mutant strain when mixed with a compatible wild type or mutant strain (Fig. 43). All the colony morphology and plate mating assay data support the observations made in Chapter II of this dissertation, that Hsl7 plays a role in regulating the filamentous response to SLAD but not the mating response.

All previous data suggest that the independent $\Delta h s l 7$ mutant strains are affected in cell length and the filamentous response to low ammonium conditions, but not in growth rate or the mating response. However, the production of cross walls was only observed in the $M U M$ background as the $\Delta_{810-2832} h s l 7$ mutant strains did not exhibit cross 

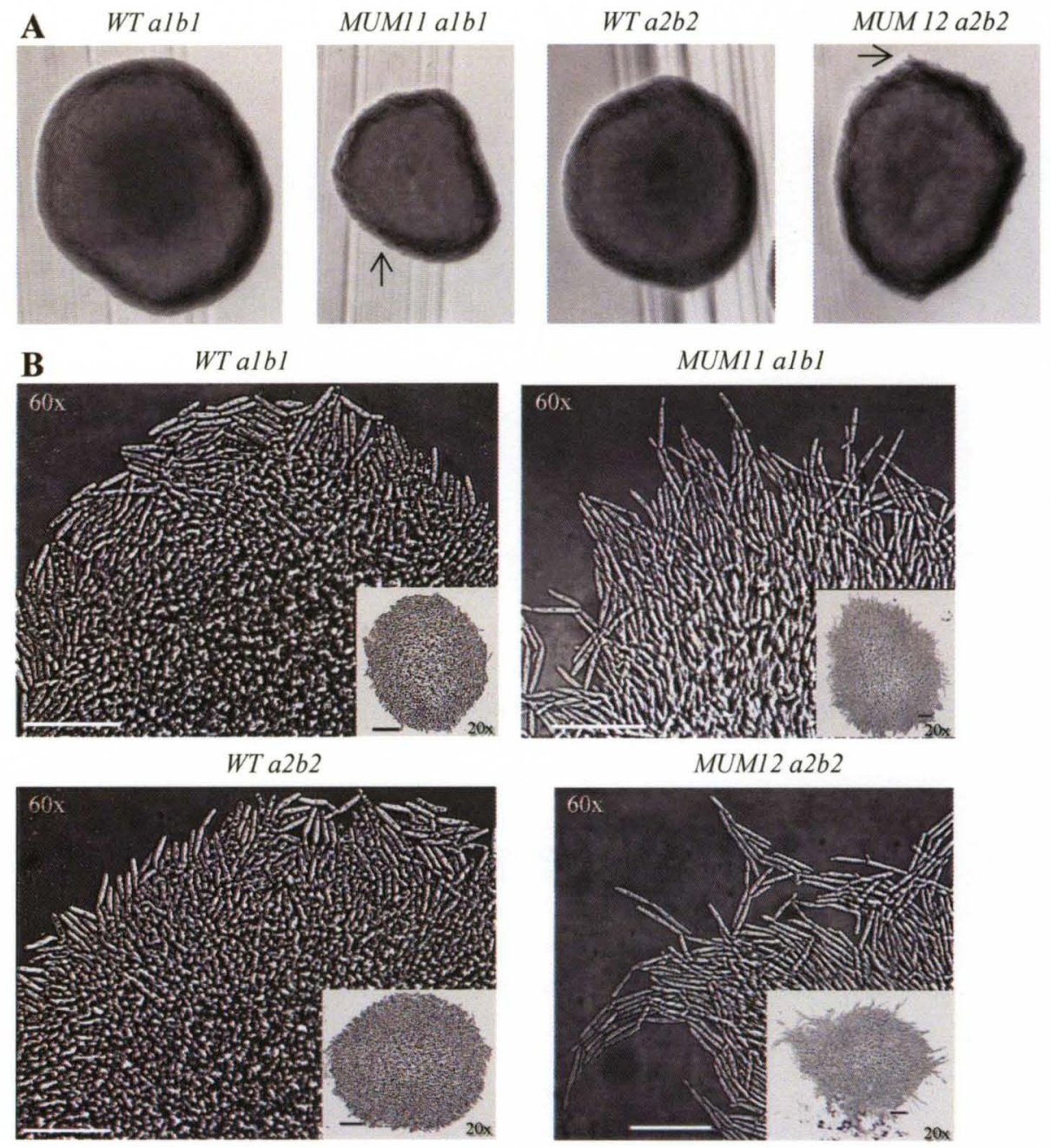

Figure 41: $M U M$ strains exhibit a directional growth phenotype. Grown on rich media, $M U M$ colonies exhibit small protrusions of cells extending away from the colony edge (A, see arrows). Examination of micro-colonies of the MUM mutant strains indicated "directional growth" where groups of parallel cells grow in a specific direction different from another group of cells (B). Scale bars, $50 \mu \mathrm{m}-20 \mathrm{x}$ inset image, $40 \mu \mathrm{m}-60 \mathrm{x}$ image.

wall formation. Specifically, MUM11 displayed sensitivity to cell wall antagonists. This was seen when the $M U M$ strains were spotted on media containing the cell stressors 50 $\mu \mathrm{M}$ CFW (chitin antagonist), $15 \mu \mathrm{g} / \mathrm{mL} \mathrm{CR}$ ( $\beta$-glucan antagonist), or the osmotic stressor $1 \mathrm{M} \mathrm{NaCl}$. When spotted on media containing CR or CFW, the MUMII strain showed a delay or outright lack of growth, respectively, at 48 hours after inoculation (Fig. 44A). However, at 72 hours the growth of $M U M 11$ on CR had recovered, but no 

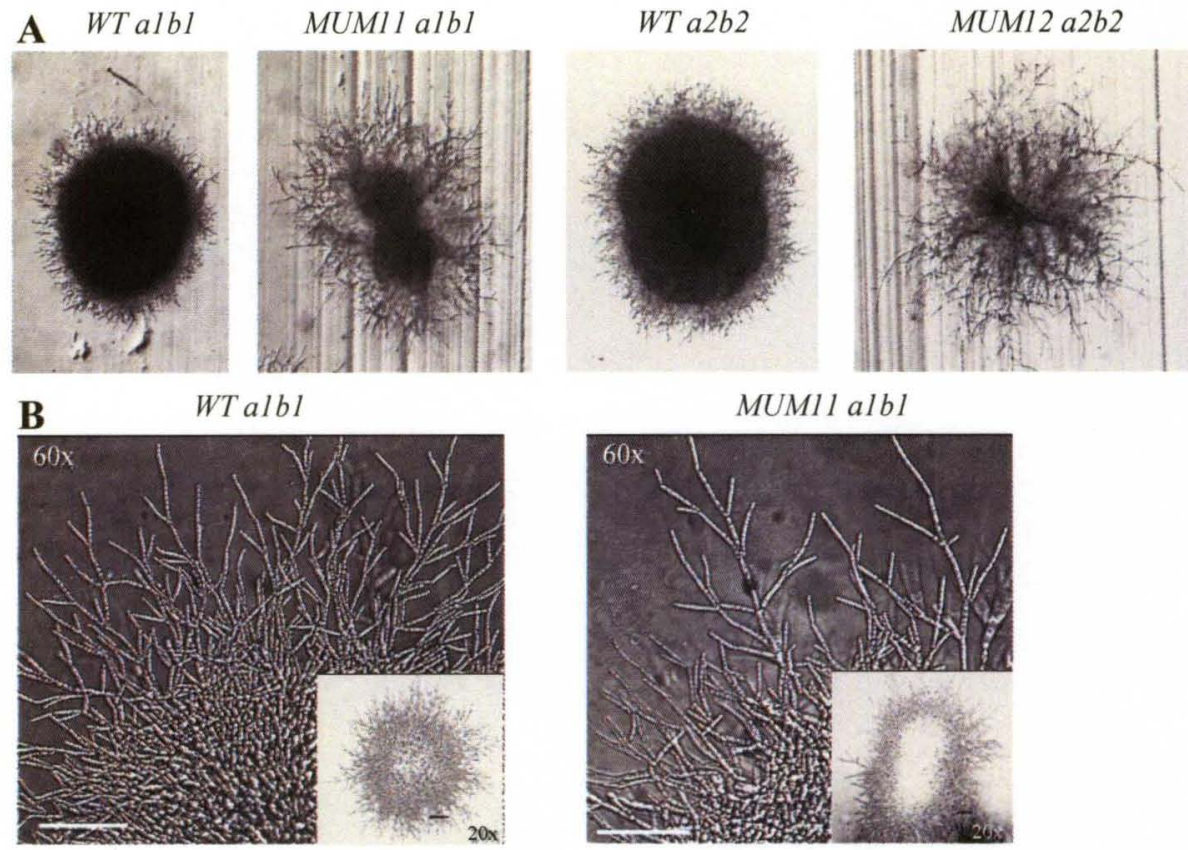

MUMIl albl

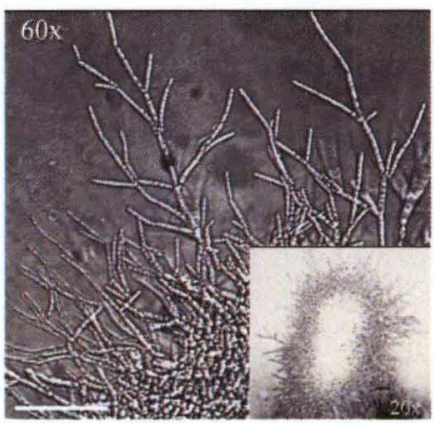

WT $a 2 b 2$

MUM12 a2b2
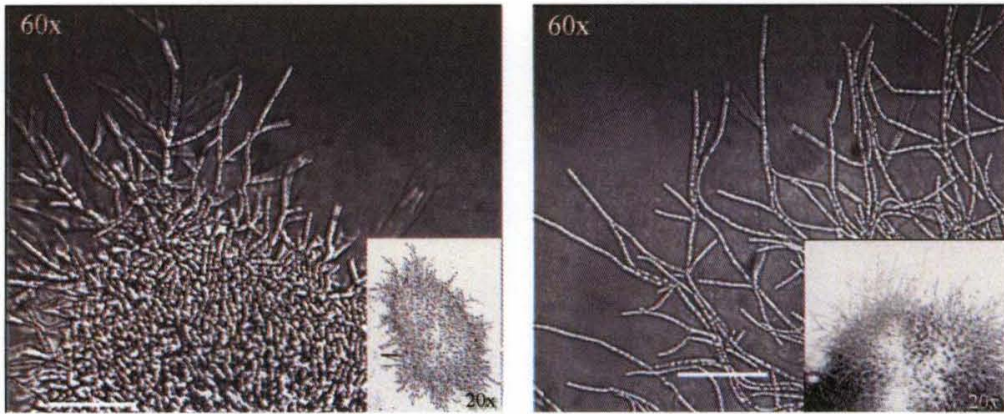

Figure 42: Filamentous response to low ammonium conditions of hsl7 mutants. MUM colonies display an increase in the ability to form filaments when grown on SLAD (A). Micro-colonies were utilized to examine filament formation at the cellular level. The MUM strains were increased in filament length and branching (B). Scale bars, $50 \mu \mathrm{m}-20 \mathrm{x}$ inset image, $40 \mu \mathrm{m}-60 \mathrm{x}$ image.

similar recovery was observed on CFW containing media. When spotted on media containing $1 \mathrm{M} \mathrm{NaCl}$, again the $M U M 11$ strain displayed a decrease in growth after 96 hours (Fig. 44A). However, it may be that the sensitivity to $1 \mathrm{M} \mathrm{NaCl}$ is due a defect in the cell wall structure as demonstrated by CFW and CR sensitivity.

To further explore the lethality of CFW, cells were grown overnight in liquid YEPS media then diluted into fresh YEPS media containing $50 \mu \mathrm{M} \mathrm{CFW}$ and viewed microscopically after 4 hours. Both wild type strains and the MUM12 strain were able to 


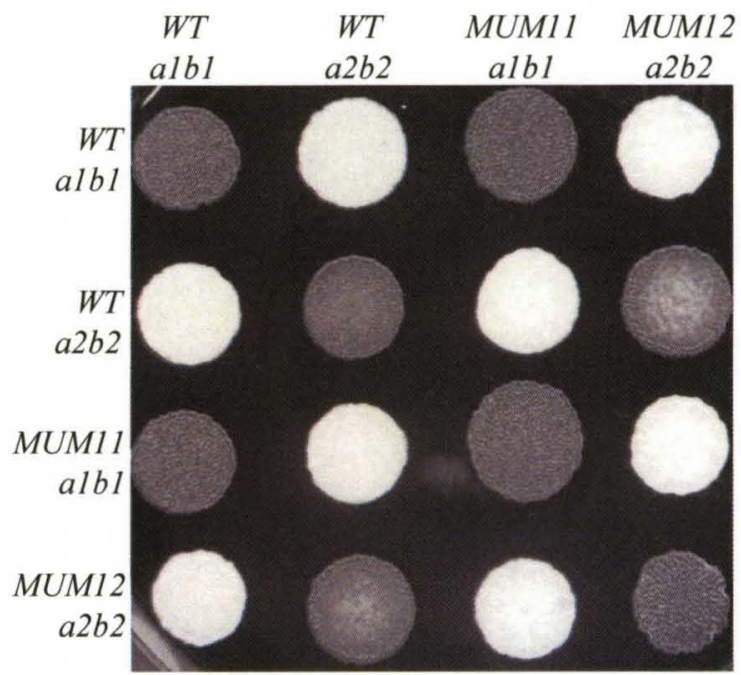

Figure 43: Plate mating
assays for the $M U M$
strains. Equal mixtures
of haploid strains of
opposite mating-type
background were plated
onto YPD plates
containing activated
charcoal. A positive
mating reaction produced
a white "fuz" phenotype
of aerial hyphae
production. See text for
description of results.

grow in the stressor media; conversely, the MUM11 strain was unable to grow.

Examination of cell morphology indicated that the wild type and MUM12 strains produced cells that were large and highly branched (Fig. 44B). Though CFW stress prevents growth of MUM11 cells, several were able to be viewed and their morphology appeared to be a group of short, thickened undivided cells with failed buds at the ends of the cell groups (Fig. 44B).

It is possible that $\mathrm{CFW}$ is weakening the cell wall of the MUM11 strain making it extremely susceptible to internal osmotic stresses that wild type cells are easily able to handle. To explore this idea the $M U M$ strains were spotted onto CFW stressing media that contained a $1 \mathrm{M}$ concentration of the osmotic stabilizer sorbitol. Sorbitol did partially rescue the growth of the MUM11 strain (Fig. 45A). The MUM11 was able to grown in CFW stressor media containing $1 \mathrm{M}$ sorbitol and the cell morphology was similar to that observed in the wild type strains, as well as in MUM12 (Fig 45B). Oddly, this specific media causes bi-polar growth of cells, where cells lose their single polar bud site selection and buds grow from both ends of the cell simultaneously. 


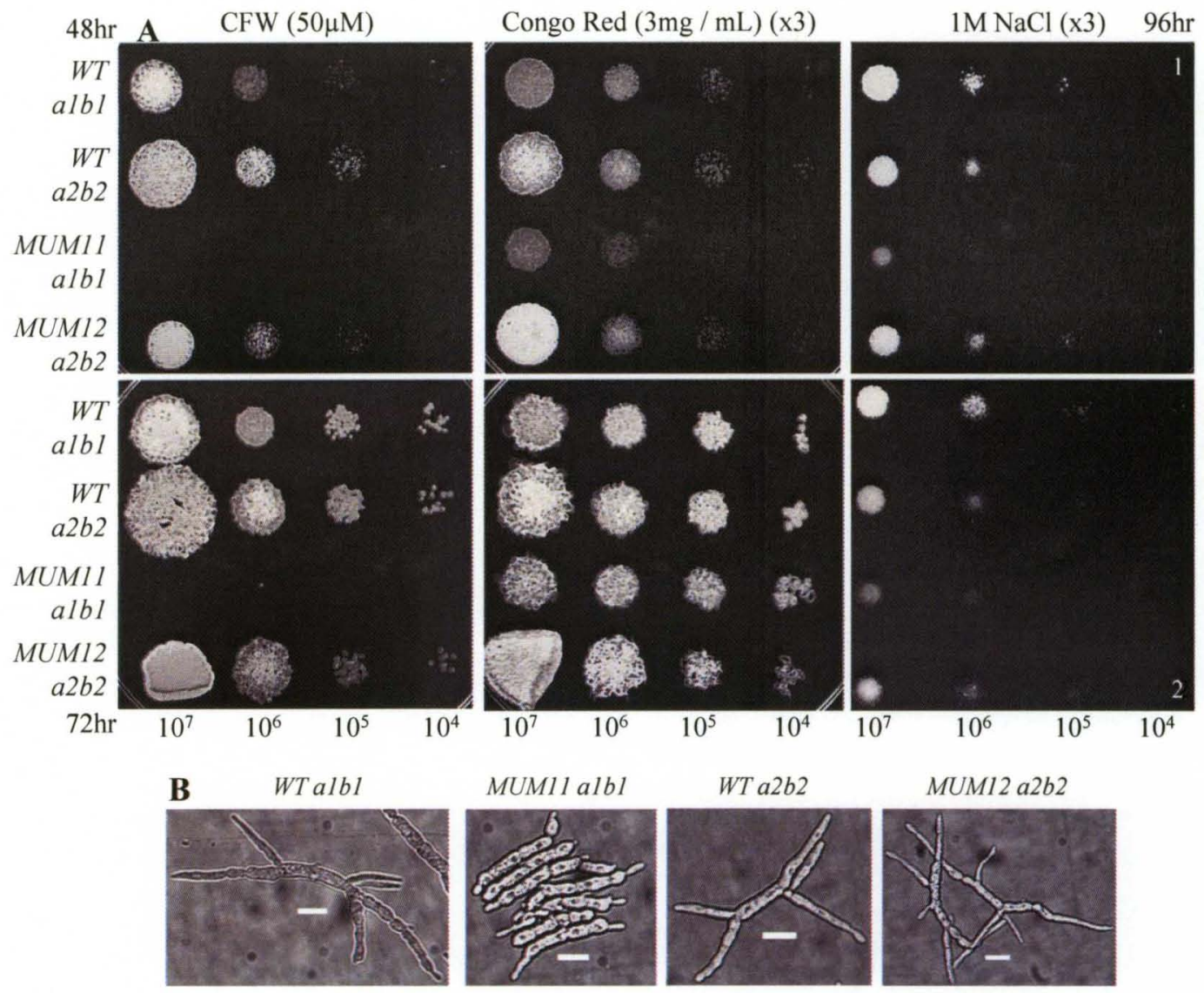

Figure 44: Cell wall stressors. Serial dilutions of the $M U M$ strains were spotted onto media containing $50 \mu \mathrm{M}$ calcofluor white (CFW; chitin antagonist), $15 \mu \mathrm{g} / \mathrm{mL}$ congo red (CR; $\beta$-glucan antagonist), or the osmotic stressor $1 \mathrm{M} \mathrm{NaCl}$. Observations were made at $48 \mathrm{hr}$ and $72 \mathrm{hr}$ for $\mathrm{CFW}$ and $\mathrm{CR}$ media, while observations were made at $96 \mathrm{hr}$ for $1 \mathrm{M} \mathrm{NaCl}$. Growth defects were only observed in MUM11 both CFW and CR media at $48 \mathrm{hr}$, though growth had rescued by $72 \mathrm{hr}$ on CR. At $96 \mathrm{hr} M U M 11$ was sensitive to $1 \mathrm{M}$ $\mathrm{NaCl}$ (A). Cell morphology was observed when the $M U M$ strains were grown in liquid media containing CFW. Again only the MUM11 strain displayed a different cell morphology from wild type strains and MUM12, and fail to grow in liquid CFW media (B). Scale bars, $10 \mu \mathrm{m}$.

The sensitivity to CFW of the MUMl1 strain raised the question of how this strain differs from the other two $\Delta h s l 7$ mutant strains previously described (Chapter II). As previously described, the $\Delta h s l 7$ mutant strains contain a complete deletion of the $h s l 7$ ORF, while the $\Delta_{810-2832} h s l 7$ mutant strains were lacking the nucleotides from 810 to the end of the ORF. In construction of the $\Delta_{810-2832} h s l 7$ construct, the first 828 nucleotides that could potentially encode a 275 amino acid truncated protein were left intact. 

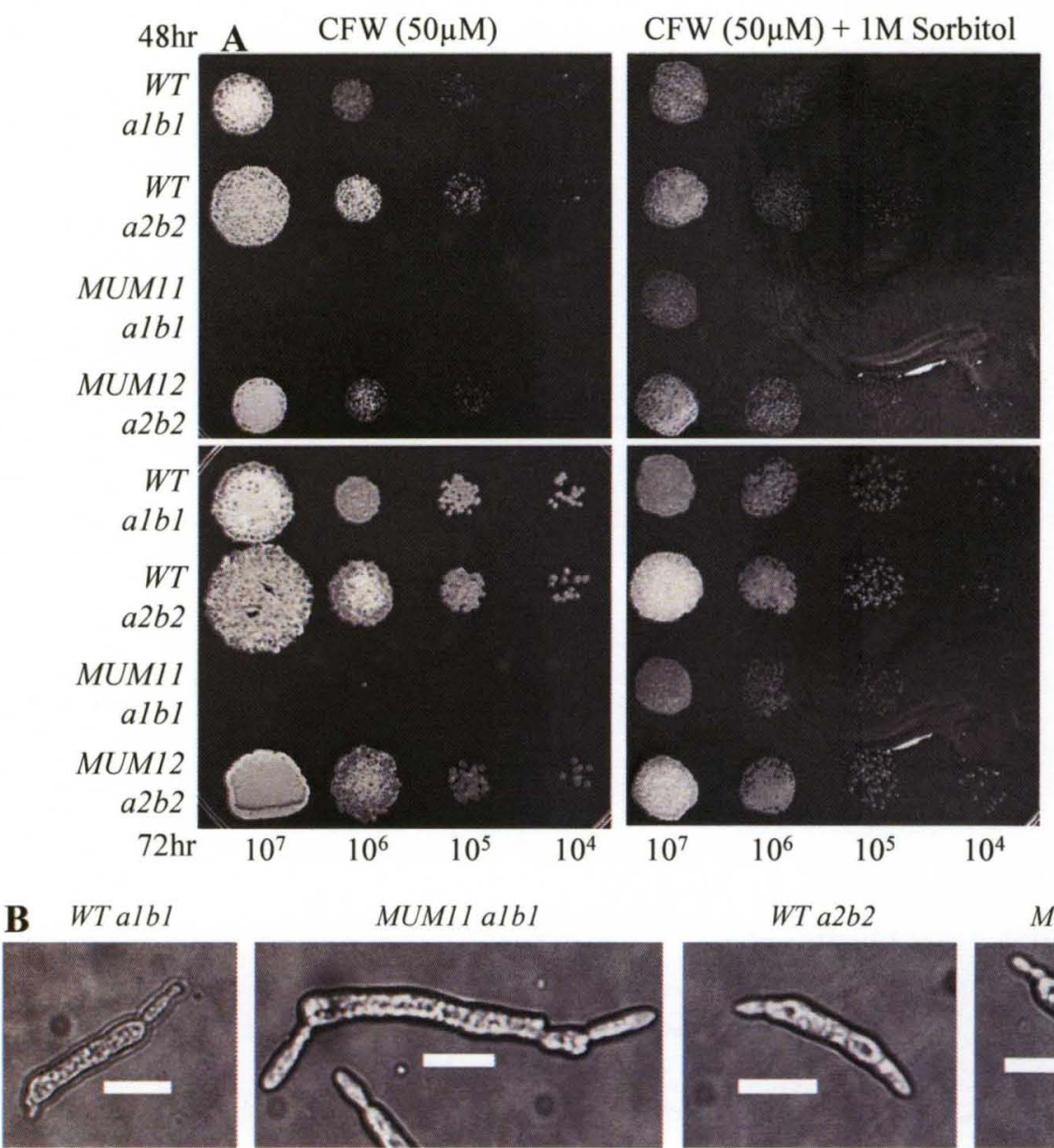

MUM12 a $2 b 2$

Figure 45: Rescue of CFW sensitivity by the osmotic stabilizer sorbitol. Serial dilutions of the $M U M$ strains were spotted onto media containing $50 \mu \mathrm{M}$ CFW and the osmotic stabilizer sorbitol partially rescued the growth defect observed in the MUM11 strain (A). Cell morphology of cells grown in liquid media containing $\mathrm{CFW}$ and $1 \mathrm{M}$ sorbitol indicated that though all cell morphology was affected in all strains, the MUM11 strain grew and was not differently affected in cell morphology compared to wild type strains and MUM12 (B). Scale bars, $10 \mu \mathrm{m}$.

Exploration of the deletion construct from the MUM11 strain indicated that MUM11 is a partial disruption of the $h s l 7$ ORF. The first 480 nucleotides of the $h s l 7$ ORF were intact and potentially encode a 159 amino acid truncated protein. It is possible that this truncated protein may be expressed and interferes with either chitin synthesis and / or localization. Eight chitin synthases have been identified in U. maydis and appear to work in coordination at the septa in yeast-like cells and hyphae (Weber, et al., 2006). Of the eight chitin synthases, Chs5 and Chs7 are most important in cell morphology and 
separation. How the MUM11 strain is sensitive to CFW stress and if it is affecting chitin synthase localization and / or activity are interesting questions that are currently being explored. Expression, as well as potential localization of the truncated protein is currently being examined.

\section{MATERIALS and METHODS}

Strains. Ustilago maydis strains utilized in this study are listed in Table 18.

TABLE 18. U. maydis strains used in this study.

\begin{tabular}{lll} 
Strain & Genotype & Reference \\
\hline Fungal Strains & & \\
FB1 & $a l b 1$ & Banuett, et al, 1989 \\
MUM11 & $a l b 1 h s l 7:: c b x^{R}$ & Dr. José Pérez-Martín \\
\hline FB2 & $a 2 b 2$ & Banuett, et al, 1989 \\
MUM12 & $a 2 b 2 h s l 7:: c b x^{R}$ & Dr. José Pérez-Martín \\
\hline
\end{tabular}

${ }^{A}$ All mutant strains were made in the wild type background strains listed.

Growth conditions, cell length, statistical analysis, staining, micro-colonies, and microscopy are described previously (Materials and methods, Chapter II). 


\section{CURRICULUM VITAE}

Name: $\quad$ Charles Benjamin Lovely

Address: $\quad 100$ Colonial Dr.

Louisville, KY 40207

DOB: $\quad$ Somerset, KY - November 13, 1977

Education:

Graduate:

2002-2010

Degree:

$\mathrm{PhD}$ in Molecular, Cellular, and Development Biology

School:

GPA:

University of Louisville

3.25

Undergraduate:

1997-2002

Degree:

School:

GPA:

B.S., Biology; B.A., Chemistry

University of Louisville

3.18

\section{Grants:}

The Richard B. and Constance L. Lewis Educational Scholarship: 2007

Project Title: Global Gene Expression in Mexico

Awards:

Conwell / Furnish Teaching Award: 2005

Department of Biology

University of Louisville

CGeMM Travel Award: 2009

Center for Genetics and Molecular Medicine

University of Louisville

Travel Award, 25 $5^{\text {th }}$ Fungal Genetic Conference: 2009

BioLegend Co.

Teaching Experience:

Microbiology, T.A.: 2002-2007

Department of Biology

University of Louisville

Chemistry, Instructor: 2007 
Department of Allied Health

Brown-Mackie College

Microbiology and Urinalysis, Instructor: 2007

Department of Allied Health

Brown-Mackie College

Microbiology, Instructor: 2008

Department of Allied Health

Brown-Mackie College

Biology Lab for Non-Majors, T.A.: 2008

Department of Biology

University of Louisville

\section{Posters}

David Smith $^{1}$, Casey Shawler ${ }^{1}$, C. Ben Lovely ${ }^{1}$, Joe Heitman ${ }^{2}$, and Michael H. Perlin ${ }^{1}$

${ }^{1}$ University of Louisville, Louisville, Kentucky, USA and ${ }^{2}$ Duke University, Durham, North Carolina, USA

To "P" or not to "P"?: The action of methylammonium permeases in fungal dimorphism depends on their ability to be phosphorylated.

Presented at the $22^{\text {nd }}$ Fungal Genetics Conference, Asilomar, CA 2003.

C. Ben Lovely, Gregory E. Shaw, and Michael H. Perlin. Department of Biology, Program on Disease Evolution, University of Louisville, Louisville, Kentucky, USA Mating Type Specific Signaling in Ustilago maydis. Presented at the $25^{\text {th }}$ Fungal Genetics Conference, Asilomar, CA 2009

C. Ben Lovely, Gregory E. Shaw, and Michael H. Perlin. Department of Biology, Program on Disease Evolution, University of Louisville, Louisville, Kentucky, USA Regulation of Morphogenesis in Response to Low Ammonium in Ustilago maydis. Presented at the Mycology Society of America Conference, Lexington, KY 2010

Gregory E. Shaw, C. Ben Lovely, and Michael H. Perlin. Department of Biology, Program on Disease Evolution, University of Louisville, Louisville, Kentucky, USA Mating Type Specific Signaling in Ustilago maydis. Presented at the Mycology Society of America Conference, Lexington, KY 2010

\section{Publications}

C. Ben Lovely and Michael H. Perlin. Department of Biology, Program on Disease Evolution, University of Louisville, Louisville, Kentucky, USA (Manuscript in Preparation) The role of Hsl7 in morphology and pathogenicity in the plant pathogen Ustilago maydis.

Cau D. Pham, C. Ben Lovely, Zhanyang Yu, David Myers, Trisha Patel, and Michael H. Perlin. Department of Biology, Program on Disease Evolution, University of Louisville, Louisville, Kentucky, USA (Manuscript in Preparation) 
Haplo-insufficiency in Ustilago maydis reduces pathogenicity. 